Supporting Information for

\title{
The Radical-Trapping Antioxidant Activity of Copper and Nickel bis(Semithiocarbazone) Complexes Underlies Their Potency as Inhibitors of Ferroptotic Cell Death
}

\author{
Omkar Zilka, Jia-Fei Poon and Derek A. Pratt* \\ Department of Chemistry and Biomolecular Sciences, University of Ottawa, Ottawa, Ontario K1N 6N5, Canada \\ *dpratt@uottawa.ca \\ Table of Contents
}

Supplemental Experimental Details......................................................................

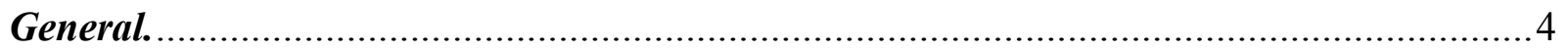

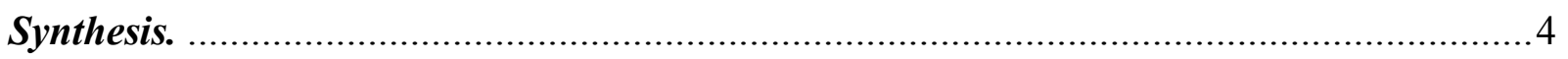

Inhibited Autoxidations Monitored by $U$ V-Vis in the Absence of PBD-BODIPY. ...............8

Single-Ion Monitoring Mass Spectrometry (SIM-MS). ................................................ 8

Preparative Scale CuATSM-Inhibited Autoxidation of THF. ....................................... 9

Figure S1. UV-Vis spectrum of reaction intermediate $\boldsymbol{e}(20 \mu \mathrm{g} / \mathrm{mL})$ isolated from a preparative CuATSM-inhibited autoxidation of THF. Spectrum recorded in the autoxidation solution of 3:1 $\mathrm{PhCl}$ :THF for direct comparison to the spectra recorded during the

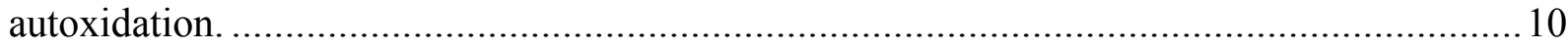

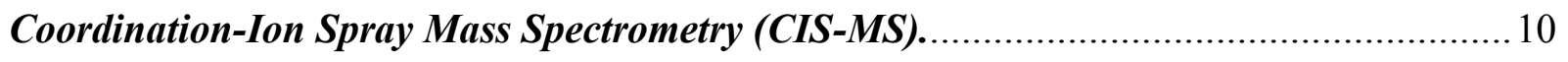

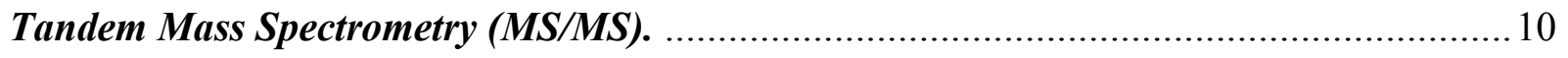

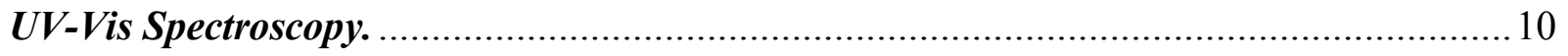

Supplemental THF/PBD-BODIPY Co-autoxidation Data.......................................... 11

Figure S2. Uncropped representative traces of co-autoxidations of $\mathrm{THF}: \mathrm{PhCl}$ (1:3) and PBDBODIPY $(10 \mu \mathrm{M})$ initiated with AIBN $(6 \mathrm{mM})$ at $37^{\circ} \mathrm{C}$ and inhibited by $\mathrm{CuATSM}_{2}, \mathrm{PMC}$, or 2,6-di-tert-butyl-4-methylphenol (BHT) at the indicated concentrations. Full conditions

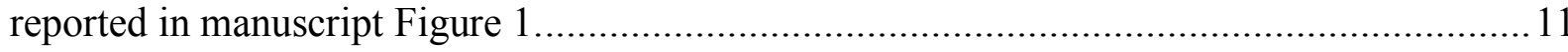

Supplemental Egg PC/STY-BODIPY Co-autoxidation Data .......................................... 12

Figure S3. Representative co-autoxidations of STY-BODIPY $(1 \mu \mathrm{M})$ and egg PC liposomes $(1 \mathrm{mM})$ in PBS initiated with AAPH $(\mathbf{A}, 1 \mathrm{mM})$ at $37^{\circ} \mathrm{C}$ and inhibited by copper and nickel bis(thiosemicarbazone) complexes (B,C). Full conditions reported in manuscript Figure $2 \ldots 12$ Figure S4. Summary of inhibition rate constants (A) and radical-trapping stoichiometry (B) obtained from co-autoxidations of STY-BODIPY $(1 \mu \mathrm{M})$ and egg PC $(1 \mathrm{mM})$ in PBS initiated with DTUN $(0.2 \mathrm{mM})$ at $37^{\circ} \mathrm{C}$ and inhibited by bis(thiosemicarbazone) copper and nickel complexes with and without reductants. Representative raw data are shown in panels C-J. The 
bottom graph of each panel are expansions of the initial portion of the reactions. Full conditions are reported in manuscript Figure 2.

UV-Vis Spectral Data for Reduction of Metal Bis(Thiosemicarbazone) Complexes with Ascorbate.

Figure S5. UV-Vis spectra of indicated complexes $(16 \mu \mathrm{M})$ in the indicated solvents with 1.6 $\%$ DMSO recorded at $37^{\circ} \mathrm{C}$ (black) and with added ascorbic acid ( $64 \mu \mathrm{M}, 4$ eq; red, scan after

2 mins; blue, scan after 10 mins). 15

Ferroptosis Cell Rescue Assays in HT-22 Cells............................................................. 16

Figure S6. (A-D) Rescue of RSL3-induced ferroptosis in HT22 cells (6000 cells in $100 \mu \mathrm{L}$; $150 \mathrm{nM}$ RSL3 for $5 \mathrm{~h}$ ) and associated $\mathrm{EC}_{50}$ values (n.e. = not effective). Supplemental to manuscript Figure 3.

UPLC Chromatograms of Copper Bis(thiosemicarbazone) Complexes.

Figure S7. UPLC-PDA TIC chromatograms of $\mathrm{Cu}$ complexes $\left(10 \mu \mathrm{M}\right.$ in $\left.1: 1 \mathrm{MeCN}: \mathrm{H}_{2} \mathrm{O}\right)$ :

(A) blank injection, (B) CuATSM, (C) CuGTSM, (D) CuATSM2, (E) CuATSP, (F)

CuATSM-d6, (G) CuATSM2-d6. For details on conditions, see general section above. 17

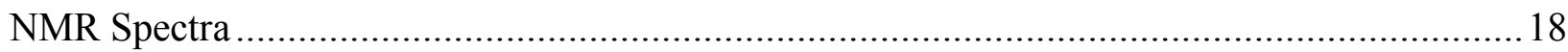

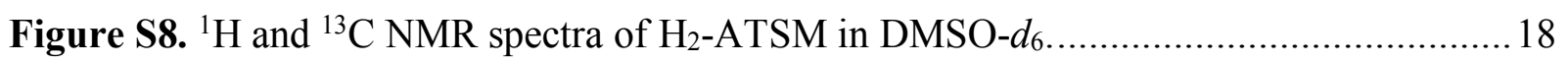

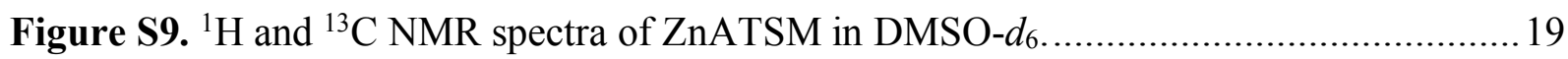

Figure S10. ${ }^{1} \mathrm{H}$ NMR spectrum of NiATSM in DMSO- $d_{6}$ and $\mathrm{CDCl}_{3}$ used to calculate $\alpha 2 \mathrm{H}$

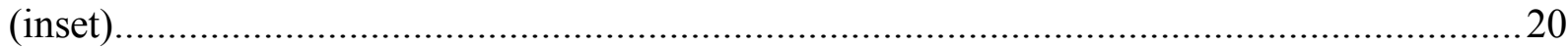

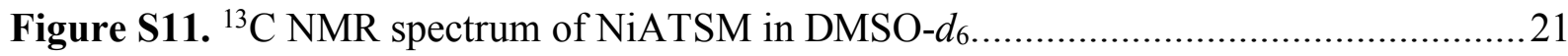

Figure S12. ${ }^{1} \mathrm{H}$ NMR spectrum of NiATSM in 1:1 DMSO- $d_{6}: \mathrm{MeOD}_{4}$ to verify $\mathrm{H} / \mathrm{D}$ exchange. 21

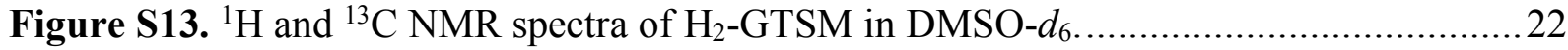

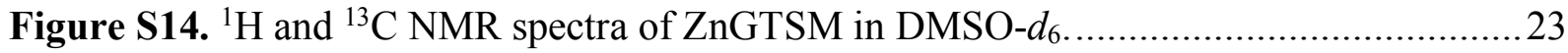

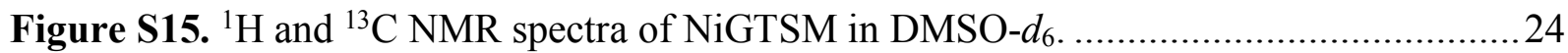

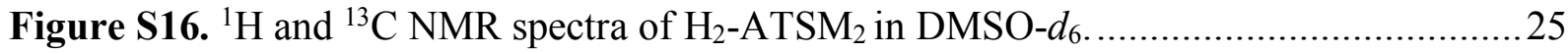

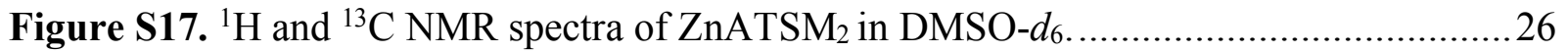

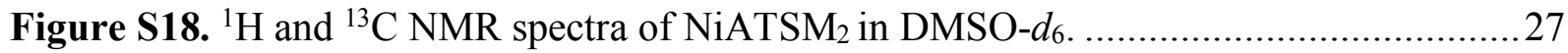

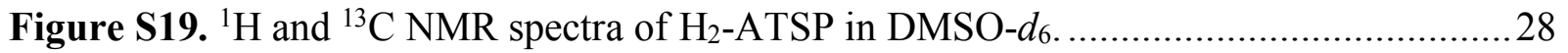

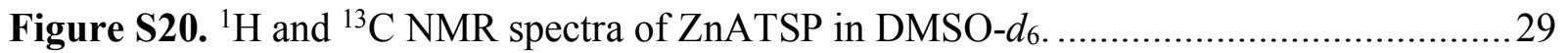

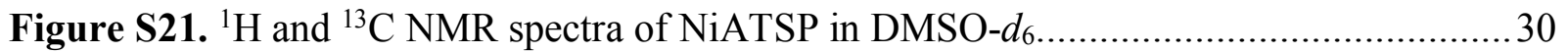

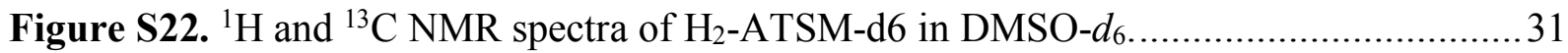

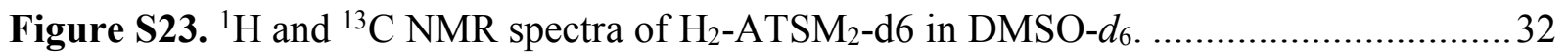

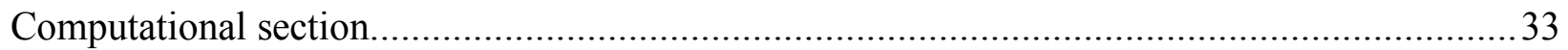

Figure S24. HAT and addition peroxyl radicals to truncated analogue of CuATSM.............33

Table S1. Benchmark of various methods ......................................................................... 34 
Table S2. Benchmark of various methods.

Figure S25. Free energy profile of radical-trapping for truncated analogues derived from

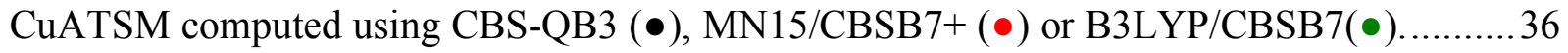

Figure S26. MN15/CBSB7+ computed free energies (in $\mathrm{kcal} / \mathrm{mol}$ ) predict that radical addition to $\mathrm{NiATSM}_{2}$ in THF is less reversible than to CuATSM 2.

Figure S27. MN15/CBSB7+ computed free energies (in $\mathrm{kcal} / \mathrm{mol}$ ) for substitution at the Satom of CuATSM in THF at $298 \mathrm{~K}$ and subsequent inner-sphere electron transfer to yield a sulfinyl radical.

Figure S28. Predicted UV-Vis spectra of CuATSM (top, black) and $\mathrm{ATSM}_{2}$ (bottom, black) and the presumed intermediates formed therefrom (red) during the inhibited autoxidations. TD-DFT calculations of the first 30 excited states were carried out at the MN15/CBSB7+ level

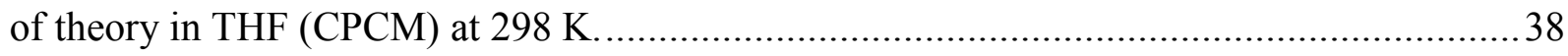

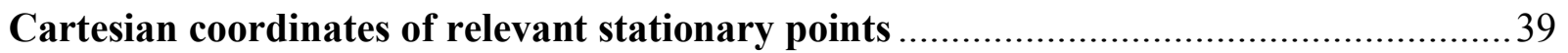

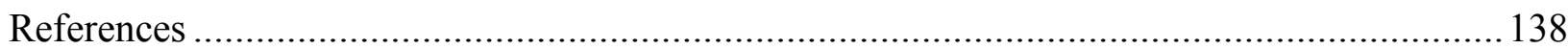




\section{Supplemental Experimental Details}

General. All chemicals were obtained from commercial sources and used without further purification, unless otherwise indicated. Solvents, including water used in washing precipitates, were LC-grade in all cases to minimize trace metal contaminations. Solvents indicated as " $\mathrm{N}_{2}$ purged" were sparged separately from the reactions for at least 20 minutes. Phosphate buffered saline (PBS) was $12 \mathrm{mM}$ phosphate, $150 \mathrm{mM} \mathrm{NaCl}, \mathrm{pH}$ 7.4, and passed over a Chelex-100 column preequilibrated at $\mathrm{pH}$ 7.4. NMR spectra were recorded on a Bruker AVANCE III HD $600 \mathrm{MHz}$ spectrometer at $298 \mathrm{~K}$. Chemical shifts $(\delta)$ are reported in parts per million $(\mathrm{ppm})$ referenced internally to the residual solvent peak. Coupling constants are reported in Hertz $(\mathrm{Hz})$. The following abbreviations indicate peak multiplicities: $\mathrm{s}$, singlet; $\mathrm{d}=$ doublet; $\mathrm{t}=$ triplet; $\mathrm{q}=$ quartet; $\mathrm{m}=$ multiplet; $\mathrm{br}=$ broad. High resolution mass spectra were recorded by electrospray ionization (ESI) in positive mode on a Micromass Q-TOF II mass spectrometer. UPLC analysis were carried out on a Waters Acquity H-Class instrument fitted with a $2.5 \mathrm{~mm}$ x $50 \mathrm{~mm} \mathrm{C18} \mathrm{column}$ and PDA UV-Vis detector. Mobile phase (20 mins total): $t=0$ to $10 \mathrm{~min}, 95: 5$ to 5:95 $\mathrm{H}_{2} \mathrm{O}: \mathrm{MeCN} ; t=10$ to $13 \mathrm{~min}$, hold at 5:95 $\mathrm{H}_{2} \mathrm{O}: \mathrm{MeCN} ; t=13$ to $16 \mathrm{~min}, 5: 95$ to $95: 5 \mathrm{H}_{2} \mathrm{O}: \mathrm{MeCN} ; t=16$ to $20 \mathrm{~min}$, hold at 95:5 $\mathrm{H}_{2} \mathrm{O}: \mathrm{MeCN}$.

\section{Synthesis.}

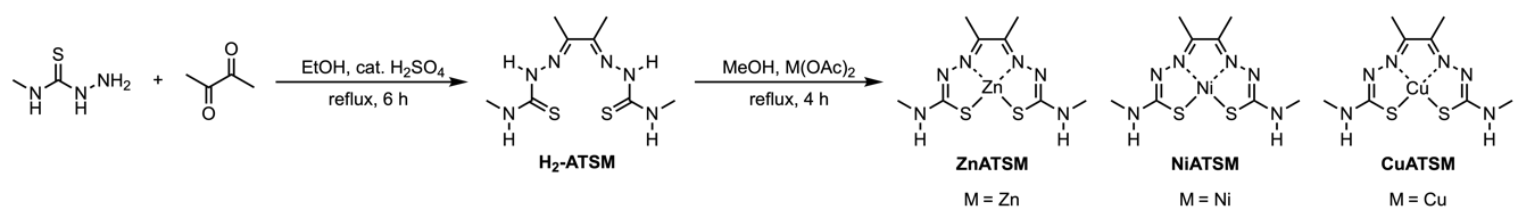

H2-ATSM. ${ }^{1,2}$ A stirred suspension of 4-methylthiosemicarbazide (2.40 g, $22.8 \mathrm{mmol}, 2$ eq) in $\mathrm{N}_{2}$ purged ethanol $(50 \mathrm{~mL})$ with 1 drop of concentrated $\mathrm{H}_{2} \mathrm{SO}_{4}$ was heated to reflux under $\mathrm{N}_{2}$. Once fully dissolved, a solution of 2,3-butanedione (1 mL, $11.4 \mathrm{mmol}, 1 \mathrm{eq})$ in ethanol (2 mL) was added dropwise, which led to the formation of a cream-coloured precipitate. The suspension was vigorously stirred for $6 \mathrm{~h}$ then cooled to $4^{\circ} \mathrm{C}$. The precipitate was collected by filtration and successively washed with cold water $(2 \times 10 \mathrm{~mL})$, ethanol $(2 \times 10 \mathrm{~mL})$ and diethyl ether $(2 \times 5$ $\mathrm{mL})$. Trace solvent was removed on high vacuum. Cream-coloured solid $(2.55 \mathrm{~g}, 86 \%) .{ }^{1} \mathrm{H}$ NMR (600 MHz, DMSO) $\delta 10.22$ (br s, 2H), 8.38 (br s, 2H), $3.02(\mathrm{~d}, J=4.8,6 \mathrm{H}), 2.20(\mathrm{~s}, 6 \mathrm{H}) .{ }^{13} \mathrm{C} \mathrm{NMR}$ $(151 \mathrm{MHz}, \mathrm{DMSO}) \delta 178.45,148.00,31.24,11.70$. HRMS (ESI) calcd for $[\mathrm{M}+\mathrm{Na}]^{+} \mathrm{C}_{8} \mathrm{H}_{16} \mathrm{~N}_{6} \mathrm{~S}_{2} \mathrm{Na}$ 283.0776, found 283.0749.

M-ATSM. $^{1,3}$ A stirred suspension of $\mathrm{H}_{2}$-ATSM $(0.52 \mathrm{~g}, 2 \mathrm{mmol}, 1 \mathrm{eq})$ and a metal acetate $\left(\mathrm{Zn}(\mathrm{OAc})_{2}, 0.40 \mathrm{~g}, 2.2 \mathrm{mmol}, 1.1 \mathrm{eq} ; \mathrm{Ni}(\mathrm{OAc})_{2} \bullet 4 \mathrm{H}_{2} \mathrm{O}, 0.55 \mathrm{~g}, 2.2 \mathrm{mmol}, 1.1 \mathrm{eq} ; \mathrm{Cu}(\mathrm{OAc})_{2}, 0.40\right.$ $\mathrm{g}, 2.2 \mathrm{mmol}, 1.1 \mathrm{eq})$ in $\mathrm{N}_{2}$-purged methanol $(20 \mathrm{~mL})$ were heated to reflux with vigorous stirring for $4 \mathrm{~h}$ under $\mathrm{N}_{2}$, then cooled to $4^{\circ} \mathrm{C}\left(-20^{\circ} \mathrm{C}\right.$ overnight for NiATSM). The precipitate was collected by filtration and successively washed with cold water $(2 \times 10 \mathrm{~mL})$, ethanol $(2 \times 10 \mathrm{~mL})$ and diethyl ether $(2 \times 10 \mathrm{~mL})$. Trace solvent was removed on high vacuum. 
ZnATSM. Yellow solid (551 mg, 85\%). ${ }^{1} \mathrm{H}$ NMR (600 MHz, DMSO) $\delta 7.18$ (br s, 2H), 2.82 (s, $6 \mathrm{H}), 2.20(\mathrm{~s}, 6 \mathrm{H}) .{ }^{13} \mathrm{C}$ NMR $(151 \mathrm{MHz}, \mathrm{DMSO}) \delta 175.76,145.41,29.19,13.91$. HRMS (ESI) calcd for $[\mathrm{M}+\mathrm{H}]^{+} \mathrm{C}_{8} \mathrm{H}_{15} \mathrm{~N}_{6} \mathrm{~S}_{2} \mathrm{Zn} 323.0091$, found 323.0091.

NiATSM. Dark green solid (608 mg, 96\%). ${ }^{1} \mathrm{H}$ NMR (600 MHz, DMSO) $\delta 7.69$ (br s, 2H), 2.78 (d, $J=4.2 \mathrm{~Hz}, 6 \mathrm{H}), 1.94$ (br s, 6H). ${ }^{13} \mathrm{C}$ NMR (151 MHz, DMSO) $\delta 176.12,155.57,32.18$, 13.85. HRMS (ESI) calcd for $[\mathrm{M}+\mathrm{H}]^{+} \mathrm{C}_{8} \mathrm{H}_{15} \mathrm{~N}_{6} \mathrm{NiS}_{2} 317.0153$, found 317.0159 .

CuATSM. Dark brown solid (581 mg, 90\%). UV-Vis (Solvent B) 307, $463 \mathrm{~nm}$. UPLC-PDA $t_{\mathrm{R}}=$ 5.38 min. HRMS (ESI) calcd for $[\mathrm{M}+\mathrm{H}]^{+} \mathrm{C}_{8} \mathrm{H}_{15} \mathrm{CuN}_{6} \mathrm{~S}_{2} 322.0096$, found 322.0088 .

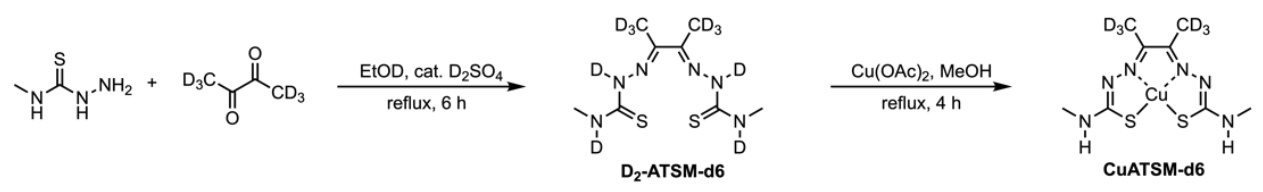

D $_{2}$-ATSM-d 6 . Prepared by the procedure used for $\mathrm{H}_{2}$-ATSM using 4-methylthiosemicarbazide $(0.316 \mathrm{~g}, 3 \mathrm{mmol}$, $2 \mathrm{eq})$ in $\mathrm{N}_{2}$-purged ethanol-OD $(4 \mathrm{~mL}), 2,3$-butanedione-d6 (138 $\mathrm{mg}, 1.5 \mathrm{mmol}$, 1 eq, CDN Isotopes, $99.7 \%$ D-incorporation) and concentrated $\mathrm{D}_{2} \mathrm{SO}_{4}$ (1 drop) in a sealed tube in an $80^{\circ} \mathrm{C}$ bath. Cream coloured solid (361 mg, 89\%). ${ }^{1} \mathrm{H}$ NMR $\left(600 \mathrm{MHz}, \mathrm{DMSO}+\right.$ drop $\left.\mathrm{H}_{2} \mathrm{O}\right) \delta$ $10.18(\mathrm{~s}, 2 \mathrm{H}), 8.39(\mathrm{~m}, 2 \mathrm{H}), 3.01(\mathrm{~d}, J=4.2 \mathrm{~Hz}, 6 \mathrm{H}) .{ }^{13} \mathrm{C}$ NMR $\left(151 \mathrm{MHz}, \mathrm{DMSO}+\right.$ drop $\left.\mathrm{H}_{2} \mathrm{O}\right) \delta$ $178.61,148.30,31.47,11.16$ (sext, $J=19.5 \mathrm{~Hz}$ ). HRMS (ESI, MeOH) calcd for $[\mathrm{M}+\mathrm{Na}]^{+}$ $\mathrm{C}_{8} \mathrm{H}_{10} \mathrm{D}_{6} \mathrm{~N}_{6} \mathrm{~S}_{2} \mathrm{Na} 289.1152$, found 289.1158 .

CuATSM-d 6 . Prepared by the procedure used for M-ATSM with $\mathrm{D}_{2}-\mathrm{ATSM}_{-} \mathrm{d}_{6}(107 \mathrm{mg}, 0.4$ $\mathrm{mmol}, 1 \mathrm{eq}), \mathrm{Cu}(\mathrm{OAc})_{2}(74 \mathrm{mg}, 0.408 \mathrm{mmol}, 1.02 \mathrm{eq})$ and methanol $(4 \mathrm{~mL})$ in a sealed tube. Dark brown solid (126 mg, 96\%). UV-Vis (Solvent B) 307, $463 \mathrm{~nm}$. UPLC-PDA $t_{\mathrm{R}}=5.34 \mathrm{~min}$. HRMS (ESI) calcd for $[\mathrm{M}+\mathrm{H}]^{+} \mathrm{C}_{8} \mathrm{H}_{9} \mathrm{D}_{6} \mathrm{CuN}_{6} \mathrm{~S}_{2} 328.0472$, found 328.0467 .

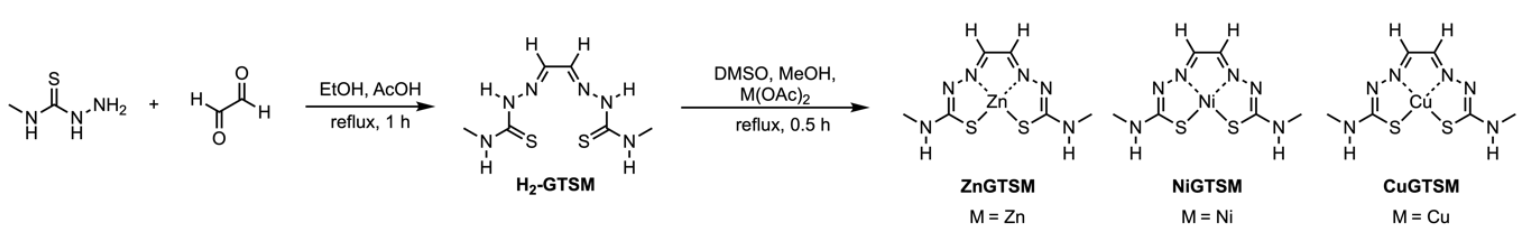

$\mathbf{H}_{2}$-GTSM. ${ }^{1,4,5}$ A stirred suspension of 4-methylthiosemicarbazide $(2.10 \mathrm{~g}, 20 \mathrm{mmol}, 2 \mathrm{eq})$ in $\mathrm{N}_{2}$ purged ethanol $(50 \mathrm{~mL})$ was heated to reflux under $\mathrm{N}_{2}$. Once fully dissolved, a solution of glyoxal $(40 \%$ aq, $1.15 \mathrm{~mL}, 10 \mathrm{mmol}, 1 \mathrm{eq})$ in ethanol $(5 \mathrm{~mL})$ was added dropwise followed by acetic acid $(1 \mathrm{~mL})$, which led to the formation of a cream-coloured precipitate. The mixture was vigorously stirred for $1 \mathrm{~h}$ then cooled to $4^{\circ} \mathrm{C}$. The precipitate was collected by filtration and successively washed with cold water $(2 \times 5 \mathrm{~mL})$ and methanol $(2 \times 5 \mathrm{~mL})$. The solid was recrystallized from $\mathrm{DMSO} /$ methanol solution at ambient temperature and the solid collected and washed as above. Trace solvent was removed on high vacuum. Colourless solid (1.83 g, 79\%). ${ }^{1} \mathrm{H}$ NMR (600 MHz, DMSO) $\delta 11.76$ (br s, 2H), 8.49 (br s, 2H), 7.72 (br s, 2H), 2.96 (s, $6 \mathrm{H}) .{ }^{13} \mathrm{C}$ NMR $(151 \mathrm{MHz}$, DMSO) $\delta 177.51,140.03,30.92$. HRMS (ESI) calcd for $[\mathrm{M}+\mathrm{Na}]^{+} \mathrm{C}_{6} \mathrm{H}_{12} \mathrm{~N}_{6} \mathrm{~S}_{2} \mathrm{Na} 255.0463$, found 255.0481 . 
M-GTSM. ${ }^{1,3}$ To a solution of $\mathrm{H}_{2}$-GTSM (116 mg, $0.5 \mathrm{mmol}, 1$ eq) in $\mathrm{N}_{2}$-purged DMSO (2.2 mL) and methanol $(1 \mathrm{~mL})$ was added a metal acetate $\left(\mathrm{Zn}(\mathrm{OAc})_{2}, 92 \mathrm{mg}, 0.5 \mathrm{mmol}, 1 \mathrm{eq}\right.$; $\mathrm{Ni}(\mathrm{OAc})_{2} \bullet 4 \mathrm{H}_{2} \mathrm{O}, 124 \mathrm{mg}, 0.5 \mathrm{mmol}, 1$ eq; $\left.\mathrm{Cu}(\mathrm{OAc})_{2}, 91 \mathrm{mg}, 0.5 \mathrm{mmol}, 1 \mathrm{eq}\right)$ and the mixture stirred vigorously and heated at $70^{\circ} \mathrm{C}$ in a sealed tube for $15 \mathrm{~min}$, sonicated for $5 \mathrm{~min}$, and further heated for $15 \mathrm{~min}$. The solution was then cooled to ambient temperature and added dropwise to rapidly stirring cold water $(30 \mathrm{~mL})$. The precipitate was collected by filtration and successively washed with cold water $(2 \times 5 \mathrm{~mL})$, ethanol $(2 \times 2 \mathrm{~mL})$ and diethyl ether $(2 \times 2 \mathrm{~mL})$. Trace solvent was removed on high vacuum.

ZnGTSM. Product required recrystallization by dissolving crude product in a minimum of hot DMSO with sonication, then adding $c a$. 5 volumes of trifluoroethanol and cooling to $4^{\circ} \mathrm{C}$ overnight. Yellow solid (113 mg, 76\%). ${ }^{1} \mathrm{H}$ NMR (600 MHz, DMSO) $\delta 7.58$ (br s, 2H), 7.47 (br s, 2H), 2.80 (br s, 6H). ${ }^{13} \mathrm{C}$ NMR (151 MHz, DMSO) $\delta 177.85,135.03,29.27$. HRMS (ESI) calcd for $[\mathrm{M}+\mathrm{H}]^{+} \mathrm{C}_{6} \mathrm{H}_{11} \mathrm{~N}_{6} \mathrm{~S}_{2} \mathrm{Zn} 294.9778$, found 294.9766.

NiGTSM. Green solid (132 mg, 91\%). ${ }^{1} \mathrm{H}$ NMR (600 MHz, DMSO) $\delta 8.03(\mathrm{~m}, 2 \mathrm{H}), 7.04(\mathrm{~m}, 2 \mathrm{H})$, $2.79(\mathrm{~d}, 2 \mathrm{H}, J=5 \mathrm{~Hz}) .{ }^{13} \mathrm{C}$ NMR $(151 \mathrm{MHz}, \mathrm{DMSO}) \delta 177.71,144.27,32.33$. HRMS (ESI) calcd for $[\mathrm{M}+\mathrm{H}]^{+} \mathrm{C}_{6} \mathrm{H}_{11} \mathrm{~N}_{6} \mathrm{NiS}_{2} 288.9840$, found 288.9856 .

CuGTSM. Red-brown solid (136 mg, 93\%). UV-Vis (Solvent A) 312, $474 \mathrm{~nm}$. UPLC-PDA $t_{\mathrm{R}}=$ 3.63 min. HRMS (ESI) calcd for $[\mathrm{M}+\mathrm{H}]{ }^{+} \mathrm{C}_{6} \mathrm{H}_{11} \mathrm{CuN}_{6} \mathrm{~S}_{2} 293.9783$, found 293.9756.

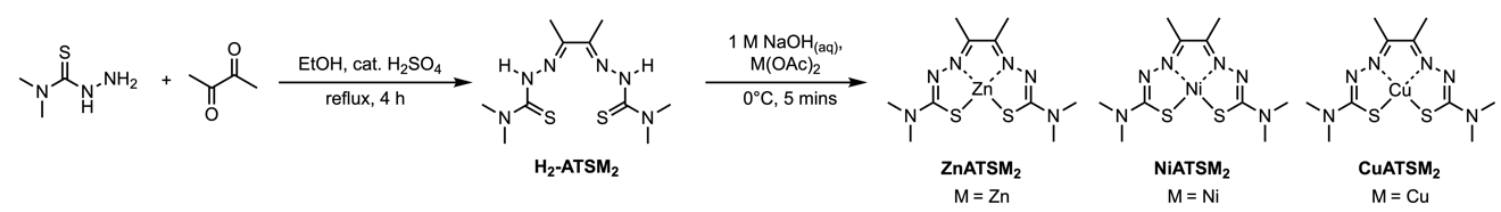

$\mathbf{H}_{2}$-ATSM ${ }_{2}{ }^{1,2}$ A stirred suspension of 4,4-dimethylthiosemicarbazide $(0.596 \mathrm{~g}, 5 \mathrm{mmol}, 2 \mathrm{eq})$ in $\mathrm{N}_{2}$-purged ethanol $(10 \mathrm{~mL})$ with 1 drop of concentrated $\mathrm{H}_{2} \mathrm{SO}_{4}$ was heated to reflux under $\mathrm{N}_{2}$. Once fully dissolved, a solution of 2,3-butanedione $(0.22 \mathrm{~mL}, 2.5 \mathrm{mmol}, 1 \mathrm{eq})$ in ethanol $(0.5 \mathrm{~mL})$ was added dropwise, which led to the formation of an orange precipitate. The suspension was vigorously stirred for $4 \mathrm{~h}$ then cooled to $4^{\circ} \mathrm{C}$. The precipitate was collected by filtration and successively washed with cold ethanol $(3 \times 5 \mathrm{~mL})$ and diethyl ether $(3 \times 5 \mathrm{~mL})$. Trace solvent was removed on high vacuum. Orange solid $(0.44 \mathrm{~g}, 61 \%) .{ }^{1} \mathrm{H}$ NMR $(600 \mathrm{MHz}, \mathrm{DMSO}) \delta 9.51(\mathrm{~s}$, 2H), 3.27 (s, 12H), 2.14 (s, 6H). ${ }^{13} \mathrm{C}$ NMR (151 MHz, DMSO) $\delta 181.74,149.77,42.25,11.08$. HRMS (ESI) calcd for $[\mathrm{M}+\mathrm{Na}]^{+} \mathrm{C}_{10} \mathrm{H}_{20} \mathrm{~N}_{6} \mathrm{~S}_{2} \mathrm{Na} 311.1089$, found 311.1067 .

M-ATSM ${ }_{2}{ }^{1,5}$ A vigorously stirred solution of $\mathrm{H}_{2}-\mathrm{ATSM}_{2}(43 \mathrm{mg}, 0.15 \mathrm{mmol}, 1 \mathrm{eq})$ in $\mathrm{N}_{2}$-purged $\mathrm{NaOH}\left(1 \mathrm{M}_{a q}, 2 \mathrm{~mL}\right)$ was cooled in an ice bath and a solution of metal acetate ( $\mathrm{Zn}(\mathrm{OAc})_{2}, 61 \mathrm{mg}$, $0.158 \mathrm{mmol}, 1.05$ eq; $\mathrm{Ni}(\mathrm{OAc})_{2} \bullet 4 \mathrm{H}_{2} \mathrm{O}, 39.3 \mathrm{mg}, 0.158 \mathrm{mmol}, 1.05 \mathrm{eq} ; \mathrm{Cu}(\mathrm{OAc})_{2}, 28.7 \mathrm{mg}, 0.158$ mmol, 1.05 eq) in $\mathrm{N}_{2}$-purged water $(0.5 \mathrm{~mL})$ was added dropwise and the mixture stirred for 5 mins. In the case of $\mathrm{Cu}(\mathrm{OAc})_{2}$ and $\mathrm{Zn}(\mathrm{OAc})_{2}$, the mixture was neutralized with aqueous $\mathrm{HCl}(1$ M). The precipitate was collected by filtration and washed with cold water $(2 \times 5 \mathrm{~mL})$. Trace solvent was removed on high vacuum. 
ZnATSM2. Orange-yellow solid (23 mg, 44\%). ${ }^{1} \mathrm{H}$ NMR (600 MHz, DMSO) $\delta 3.19$ (s, 6H), 2.20 (s, 3H). ${ }^{13} \mathrm{C}$ NMR $\left(151 \mathrm{MHz}, \mathrm{CDCl}_{3}\right) \delta 175.53,158.11,39.76,18.62$. HRMS (ESI) calcd for $[\mathrm{M}+\mathrm{H}]^{+} \mathrm{C}_{10} \mathrm{H}_{19} \mathrm{~N}_{6} \mathrm{~S}_{2} \mathrm{Zn} 351.0404$, found 351.0423.

NiATSM2. Dark green solid (46 mg, 89\%). ${ }^{1} \mathrm{H}$ NMR (600 MHz, DMSO) $\delta 3.12$ (s, 6H), 1.90 (s, $3 \mathrm{H}) .{ }^{13} \mathrm{C} \mathrm{NMR}\left(151 \mathrm{MHz}, \mathrm{CDCl}_{3}\right) \delta 179.96,153.69,41.20,13.74$. HRMS (ESI) calcd for $[\mathrm{M}+\mathrm{H}]^{+}$ $\mathrm{C}_{10} \mathrm{H}_{19} \mathrm{~N}_{6} \mathrm{NiS}_{2} 345.0466$, found 345.0454 .

CuATSM 2 . Dark red solid (44 mg, 84\%). UV-Vis (Solvent B) 312, $474 \mathrm{~nm}$. UPLC-PDA $t_{\mathrm{R}}=7.94$ min. HRMS (ESI) calcd for $[\mathrm{M}+\mathrm{H}]^{+} \mathrm{C}_{10} \mathrm{H}_{19} \mathrm{CuN}_{6} \mathrm{~S}_{2} 350.0409$, found 350.0407 .

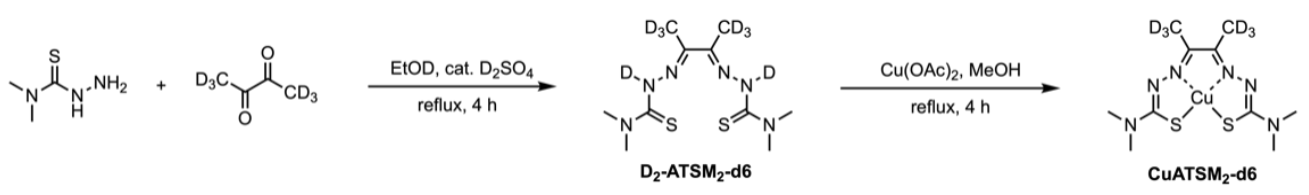

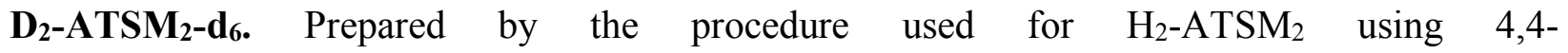
dimethylthiosemicarbazide $(0.358 \mathrm{~g}, 3 \mathrm{mmol}, 2 \mathrm{eq})$ in $\mathrm{N}_{2}$-purged ethanol-OD (4 mL), 2,3butanedione-d6 (138 mg, $1.5 \mathrm{mmol}, 1 \mathrm{eq}$, CDN Isotopes, $99.7 \%$ d-incorporation) and concentrated $\mathrm{D}_{2} \mathrm{SO}_{4}$ (1 drop) in a sealed tube in an $80^{\circ} \mathrm{C}$ bath. Orange solid $(361 \mathrm{mg}, 89 \%) .{ }^{1} \mathrm{H} \mathrm{NMR}(600 \mathrm{MHz}$, $\left.\mathrm{DMSO}+\operatorname{drop~} \mathrm{H}_{2} \mathrm{O}\right) \delta 9.51(\mathrm{~s}, 2 \mathrm{H}), 3.27(\mathrm{~s}, 12 \mathrm{H}) .{ }^{13} \mathrm{C}$ NMR $\left(151 \mathrm{MHz}, \mathrm{DMSO}+\right.$ drop $\left.\mathrm{H}_{2} \mathrm{O}\right) \delta$ $181.77,149.80,42.25,10.40$ (sext, $J=19.5 \mathrm{~Hz}$ ). HRMS (ESI, MeOH) calcd for $[\mathrm{M}+\mathrm{Na}]^{+}$ $\mathrm{C}_{10} \mathrm{H}_{14} \mathrm{D}_{6} \mathrm{~N}_{6} \mathrm{~S}_{2} \mathrm{Na}$ 317.1465, found 317.1448.

CuATSM $_{2}$-d d $_{6}$. Prepared by the procedure used for CuATSM-d6 using $\mathrm{H}_{2}-\mathrm{ATSM}_{2}$-d6 (88 mg, 0.3 mmol, 1 eq), $\mathrm{Cu}(\mathrm{OAc})_{2}(60 \mathrm{mg}, 0.33 \mathrm{mmol}, 1.1 \mathrm{eq})$ and methanol $(4 \mathrm{~mL})$. Dark red solid $(92 \mathrm{mg}$, $86 \%$ ). UV-Vis (Solvent B) $312,473 \mathrm{~nm}$. UPLC-PDA $t_{\mathrm{R}}=7.85 \mathrm{~min}$. HRMS (ESI) calcd for $[\mathrm{M}+\mathrm{H}]^{+}$ $\mathrm{C}_{10} \mathrm{H}_{13} \mathrm{D}_{6} \mathrm{CuN}_{6} \mathrm{~S}_{2} 356.0785$, found 356.0781 .

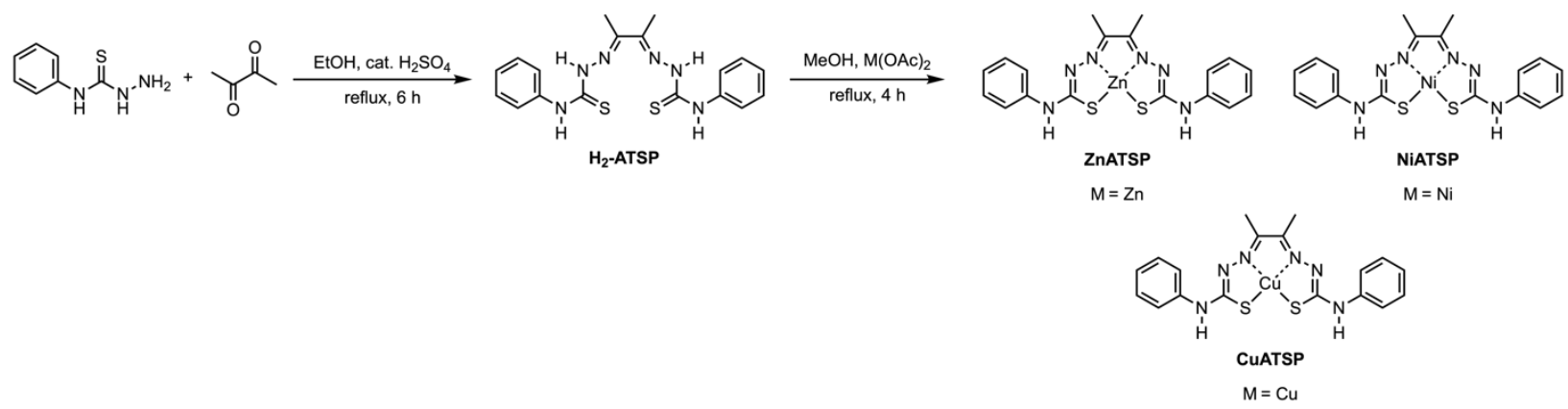

$\mathbf{H}_{2}$-ATSP. ${ }^{1,2}$ A stirred suspension of 4-phenylthiosemicarbazide (3.81 g, $22.8 \mathrm{mmol}, 2 \mathrm{eq}$ ) in $\mathrm{N}_{2}$ purged ethanol $(50 \mathrm{~mL})$ with 1 drop of concentrated $\mathrm{H}_{2} \mathrm{SO}_{4}$ was heated to reflux under $\mathrm{N}_{2}$. Once mostly dissolved, a solution of 2,3-butanedione $(1 \mathrm{~mL}, 11.4 \mathrm{mmol}, 1 \mathrm{eq})$ in ethanol $(2 \mathrm{~mL})$ was added dropwise, which led to the formation of a cream-coloured precipitate. The suspension was vigorously stirred for $6 \mathrm{~h}$ then cooled to $4^{\circ} \mathrm{C}$. The precipitate was collected by filtration and 
successively washed with cold ethanol $(2 \times 25 \mathrm{~mL}), 1: 1$ acetone:water $(2 \times 40 \mathrm{~mL})$, ethanol $(2 \times$ $25 \mathrm{~mL})$ and diethyl ether $(2 \times 25 \mathrm{~mL})$. Trace solvent was removed on high vacuum. Cream coloured solid (4.17 g, 95\%). ${ }^{1} \mathrm{H}$ NMR (600 MHz, DMSO) $\delta 10.66$ (s, 2H), 9.99 (s, 2H), 7.57 (d, $\mathrm{J}=7.2 \mathrm{~Hz}, 4 \mathrm{H}), 7.38(\mathrm{t}, \mathrm{J}=7.8 \mathrm{~Hz}, 4 \mathrm{H}), 7.22(\mathrm{t}, \mathrm{J}=7.2 \mathrm{~Hz}, 2 \mathrm{H}), 2.33(\mathrm{~s}, 6 \mathrm{H}) .{ }^{13} \mathrm{C}$ NMR $(151$ MHz, DMSO) $\delta 176.89$, 149.07, 139.00, 128.15, 125.66, 125.49, 12.15. HRMS (ESI) calcd for $[\mathrm{M}+\mathrm{Na}]^{+} \mathrm{C}_{18} \mathrm{H}_{20} \mathrm{~N}_{6} \mathrm{~S}_{2} \mathrm{Na} 407.1089$, found 407.1100.

M-ATSP. ${ }^{1,3,6}$ A stirred suspension of $\mathrm{H}_{2}$-ATSP $(0.58 \mathrm{~g}, 1.5 \mathrm{mmol}, 1 \mathrm{eq})$ and metal acetate $\left(\mathrm{Zn}(\mathrm{OAc})_{2}, 0.30 \mathrm{~g}, 1.65 \mathrm{mmol}, 1.1 \mathrm{eq} ; \mathrm{Ni}(\mathrm{OAc})_{2} \bullet 4 \mathrm{H}_{2} \mathrm{O}, 0.41 \mathrm{~g}, 1.65 \mathrm{mmol}, 1.1 \mathrm{eq} ; \mathrm{Cu}(\mathrm{OAc})_{2}\right.$, $0.30 \mathrm{~g}, 1.65 \mathrm{mmol}, 1.1 \mathrm{eq})$ in $\mathrm{N}_{2}$-purged methanol $(15 \mathrm{~mL})$ were heated to reflux with vigorous stirring for $4 \mathrm{~h}$ under $\mathrm{N}_{2}$, then cooled to $4^{\circ} \mathrm{C}\left(-20^{\circ} \mathrm{C}\right.$ with $5 \mathrm{~mL}$ water overnight for NiATSP). The precipitate was collected by filtration and successively washed with cold water $(2 \times 10 \mathrm{~mL})$, ethanol $(2 \times 10 \mathrm{~mL})$ and diethyl ether $(2 \times 10 \mathrm{~mL})$. Trace solvent was removed on high vacuum.

ZnATSP. Yellow solid (610 mg, 91\%). ${ }^{1} \mathrm{H}$ NMR (600 MHz, DMSO) $\delta 9.48(\mathrm{~s}, 2 \mathrm{H}), 7.81$ (d, J = $7.8 \mathrm{~Hz}, 4 \mathrm{H}), 7.26(\mathrm{t}, \mathrm{J}=7.8 \mathrm{~Hz}, 4 \mathrm{H}), 6.93(\mathrm{t}, \mathrm{J}=7.2 \mathrm{~Hz}, 2 \mathrm{H}), 2.34(\mathrm{~s}, 6 \mathrm{H}) .{ }^{13} \mathrm{C} \mathrm{NMR}(151 \mathrm{MHz}$, DMSO) $\delta 173.36,148.48,141.05,128.40,121.60,120.04,14.76$. HRMS (ESI) calcd for $[\mathrm{M}+\mathrm{H}]^{+}$ $\mathrm{C}_{18} \mathrm{H}_{19} \mathrm{~N}_{6} \mathrm{~S}_{2} \mathrm{Zn} 447.0404$, found 447.0405.

NiATSP. Green-brown solid (635 mg, 96\%). ${ }^{1} \mathrm{H}$ NMR (600 MHz, DMSO) $\delta 10.02$ (br s, 2H), 7.61 $(\mathrm{d}, \mathrm{J}=7.8 \mathrm{~Hz}, 4 \mathrm{H}), 7.29$ (t, J = 7.8 Hz, 4H), $7.00(\mathrm{t}, \mathrm{J}=7.8 \mathrm{~Hz}, 2 \mathrm{H}), 2.08(\mathrm{~s}, 6 \mathrm{H}) .{ }^{13} \mathrm{C}$ NMR $(151$ MHz, DMSO) $\delta 173.12,159.12,140.26,128.67,122.97,119.80,14.65$. HRMS (ESI) calcd for $[\mathrm{M}+\mathrm{Na}]^{+} \mathrm{C}_{18} \mathrm{H}_{18} \mathrm{~N}_{6} \mathrm{NiS}_{2} \mathrm{Na} 463.0286$, found 463.0293 .

CuATSP. Residual ligand removed by recrystallization by dissolving crude material in warm DMF ( $7 \mathrm{mg} / \mathrm{mL}$ ), sonicating for 10 mins, adding 3 volumes of $\mathrm{MeCN}$, and cooling at $4^{\circ} \mathrm{C}$ to yield needles. Brown solid (526 mg, 79\%). UV-Vis (Solvent C) 318, 380, $482 \mathrm{~nm}$. UPLC-PDA $t_{\mathrm{R}}=$ 7.27 min. HRMS (ESI) calcd for $[\mathrm{M}+\mathrm{H}]^{+} \mathrm{C}_{18} \mathrm{H}_{19} \mathrm{CuN}_{6} \mathrm{~S}_{2} 446.0409$, found 446.0396.

Inhibited Autoxidations Monitored by $U$ V-Vis in the Absence of PBD-BODIPY. A 3 mL quartz cuvette was equilibrated at $37^{\circ} \mathrm{C}$ in a Cary 100 spectrophotometer with tetrahydrofuran (THF, unstabilized, $0.625 \mathrm{~mL})$ and chlorobenzene $(\mathrm{PhCl}, 1.80 \mathrm{~mL})$ for 5 minutes, then baselined. AIBN ( $50 \mu \mathrm{L}$ of $0.3 \mathrm{M}$ in $\mathrm{PhCl}$ ) was added, the cuvette was inverted 5 times to mix, and then an initial scan from 700 to $280 \mathrm{~nm}$ was recorded. After 10 minutes, an aliquot of RTA ( $25 \mu \mathrm{L}$ of $0.6 \mathrm{mM}$ in DMSO, or DMSO for uninhibited reaction) was added. The cuvette was mixed, and kinetics commenced with a cycle time of $600 \mathrm{~s}$. A separate experiment without AIBN was used for the initial spectrum at $t=0 \mathrm{~s}$. The resulting spectral series were smoothed (Savitzky-Golay, 20 points), then the absorbance from the initial scan of the uninhibited reaction was subtracted from all spectra. The absorbance of AIBN does not change on the timescale of the experiments due to the low decomposition rate of $\mathrm{AIBN}$ at $37^{\circ} \mathrm{C}$. Difference spectra were calculated by subtracting each trace by the $t=0 \mathrm{~s}$ trace multiplied by the fraction of the complex remaining at the time point of the scan, which was determined from the peak at $533 \mathrm{~nm}$ for CuATSM and $545 \mathrm{~nm}$ for CuATSM , and corroborated by SIM-MS.

Single-Ion Monitoring Mass Spectrometry (SIM-MS). An autoxidation reaction was prepared identical to the UV-Vis scanning kinetics experiment described above. An aliquot $(125 \mu \mathrm{L})$ of the 
reaction was removed approximately every 35 minutes during the autoxidation and diluted into ice-cold methanol $(875 \mu \mathrm{L})$ containing $1 \%$ acetic acid. The sample was briefly vortexed and immediately infused into a Waters TQD mass spectrometer with ESI probe in positive mode at 20 $\mu \mathrm{L} / \mathrm{min}$. MS settings: Capillary voltage, $4.2 \mathrm{kV}$; Cone voltage, $50 \mathrm{~V}$; Source temperature, $100^{\circ} \mathrm{C}$; Desolvation temperature, $250^{\circ} \mathrm{C}$; Cone gas flow, $50 \mathrm{~L} / \mathrm{h}$; Desolvation gas flow, $550 \mathrm{~L} / \mathrm{h}$. The ion intensity of $m / z 322 \mathrm{Da}$ for [CuATSM $+\mathrm{H}]^{+}$, or $m / z 350 \mathrm{Da}$ for $\left[\mathrm{CuATSM}_{2}+\mathrm{H}\right]^{+}$was collected over a 30 second infusion. The intensities were corrected for background signal obtained from infusion of a sample prepared from an uninhibited reaction. A three-point standard curve verified the linearity of signal response under these infusion conditions and was used to calculate the concentration of complex in each sample. Direct infusion SIM-MS was chosen to avoid extraction of the analyte from the relatively large amount of chlorobenzene.

Preparative Scale CuATSM-Inhibited Autoxidation of THF. Solvents for purification were precooled in the refrigerator overnight. A $250 \mathrm{~mL}$ round-bottomed flask equipped with a stir bar was charged with either CuATSM (16.1 mg, 0.05 mmol, $1 \mathrm{eq})$ or CuATSM-d 6 (16.4 mg, 0.05 mmol, $1 \mathrm{eq}$ ), followed by THF (unstabilized, $12.5 \mathrm{~mL}$ ) and chlorobenzene $(37.5 \mathrm{~mL}$ ). The mixture was sonicated for 10 minutes at $37^{\circ} \mathrm{C}$ to ensure full dissolution and then the solution was sparged for 5 mins with $\mathrm{O}_{2}$. AIBN was then added (328 mg, $\left.2 \mathrm{mmol}, 40 \mathrm{eq}\right)$ and the solution stirred in the dark at $37^{\circ} \mathrm{C}$ under a balloon of $\mathrm{O}_{2}$ for $10 \mathrm{~h}$, at which point approximately half of the starting material was consumed as judged by TLC. The reaction mixture was cooled to $4^{\circ} \mathrm{C}$ over 30 mins, then the reaction mixture was decanted and mixed into $100 \mathrm{~mL}$ of cold benzene. A $3.5 \times 25 \mathrm{~cm}$ silica column was prepared in cold benzene, then the diluted reaction solution passed quickly onto the column, followed by $c a .500 \mathrm{~mL}$ of cold 1:3 ethyl acetate:methylene chloride to elute residual $\mathrm{CuATSM} / \mathrm{CuATSM}-\mathrm{d}_{6}$, AIBN, and wash away chlorobenzene. The products of the reaction were eluted with cold 1:9 methanol:methylene chloride as 2 spots $\left(\mathrm{R}_{\mathrm{f}}=0.4\right.$ in 1:9 methanol:methylene chloride) co-eluting as a yellow fraction. The fractions were concentrated in vacuo (bath temp below $10^{\circ} \mathrm{C}$ ), then for $1 \mathrm{~h}$ under high vacuum yielding product $\boldsymbol{e}$ as a reddish-yellow solid residue (10.4 mg from CuATSM; $9.2 \mathrm{mg}$ from CuATSM-d $\mathrm{d}_{6}$. The product was stored in the dark at $-20^{\circ} \mathrm{C}$ until analysis by UV-Vis spectroscopy and mass spectrometry by reconstitution in methanol at 0.5 $\mathrm{mg} / \mathrm{mL}$ by vortexing. The UV-Vis spectrum of this material was consistent with that of the intermediate observed when the inhibited autoxidation was monitored by UV-Vis (Figure S1).

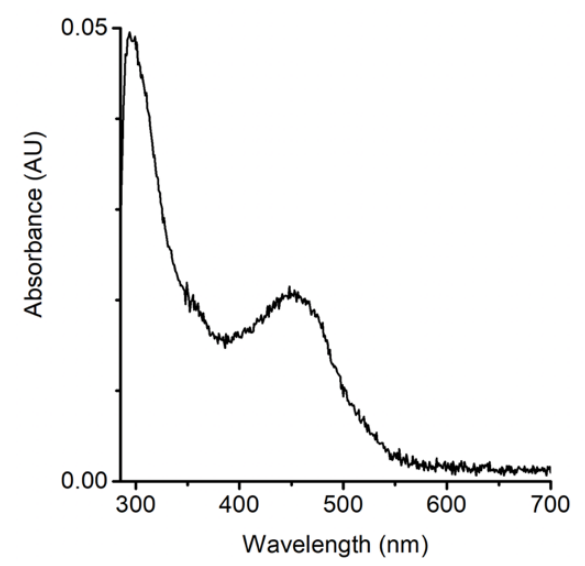


Figure S1. UV-Vis spectrum of reaction intermediate $\boldsymbol{e}(2 \mu \mathrm{g} / \mathrm{mL})$ isolated from a preparative CuATSM-inhibited autoxidation of THF. Spectrum recorded in the autoxidation solution of 3:1 $\mathrm{PhCl}$ :THF for direct comparison to the spectra recorded during the autoxidation.

Coordination-Ion Spray Mass Spectrometry (CIS-MS). ${ }^{7,8}$ A syringe pump was connected to a Waters TQD mass spectrometer using a PEEK union and short piece of PEEK tubing into the LCinlet port of the 4-way mixing valve. The valve outlet was connected to a ESI probe in positive mode with a 1-meter piece of PEEK tubing. The syringe pump was loaded with a $3 \mathrm{~mL}$ syringe of $\mathrm{MeOH}$ with a syringe filter $(0.2 \mu \mathrm{m}$ PTFE$)$ and the flow was equilibrated at $20 \mu \mathrm{L} / \mathrm{min}$ for 2 minutes to waste. Sample flow at $20 \mu \mathrm{L} / \mathrm{min}(0.1 \mathrm{mg} / \mathrm{mL}$ of intermediate $\boldsymbol{e}$ in $\mathrm{MeOH})$ was then combined with the syringe pump flow (1:1) by the instrument mixing valve fluidics and introduced into the probe. After 2 minutes of coinfusion, MS scans were collected (3 seconds per scan for 1 minute, 50 to $1500 \mathrm{Da}$ ) and the resulting spectra were combined. MS settings: Capillary voltage, $3.0 \mathrm{kV}$; Cone voltage, $60 \mathrm{~V}$; Source temperature, $70^{\circ} \mathrm{C}$; Desolvation temperature, $150^{\circ} \mathrm{C}$; Cone gas flow, $50 \mathrm{~L} / \mathrm{h}$; Desolvation gas flow, $550 \mathrm{~L} / \mathrm{h}$. The process was then repeated after equilibrating the syringe pump flow with successively higher concentrations of silver triflate (Strem, $99 \%$ ) in methanol in place of methanol until the peak for intermediate $\boldsymbol{e}$ at $\mathrm{m} / \mathrm{z} 306 \mathrm{Da}$ (from CuATSM) or $m / z 312 \mathrm{Da}$ (from CuATSM- $\mathrm{d}_{6}$ ) was mostly consumed in the resulting spectra.

Tandem Mass Spectrometry (MS/MS). A sample $(0.1 \mathrm{mg} / \mathrm{mL})$ co-infused with silver triflate (4 $\mathrm{mM}$ ) as described above was introduced in the mass spectrometer. Ions of $\mathrm{m} / \mathrm{z} 564 \mathrm{Da}$ were selected in Q1 and daughter ion mass spectra were collected at various collision energies. For Fig $6 \mathrm{H}$ (top), MS settings were: Capillary voltage, $2.0 \mathrm{kV}$; Cone voltage, $25 \mathrm{~V}$; Source temperature, $50^{\circ} \mathrm{C}$; Desolvation temperature, $50^{\circ} \mathrm{C}$; Cone gas flow, $50 \mathrm{~L} / \mathrm{h}$; Desolvation gas flow, $550 \mathrm{~L} / \mathrm{h}$; Collision gas (argon) flow, $0.1 \mathrm{~mL} / \mathrm{min}$; Collision energy, $5 \mathrm{eV}$. For Fig $6 \mathrm{H}$ (bottom), MS settings were: Capillary voltage, $3.0 \mathrm{kV}$; Cone voltage, $60 \mathrm{~V}$; Source temperature, $50^{\circ} \mathrm{C}$; Desolvation temperature, $50^{\circ} \mathrm{C}$; Cone gas flow, $50 \mathrm{~L} / \mathrm{h}$; Desolvation gas flow, $550 \mathrm{~L} / \mathrm{h}$. Collision gas (argon) flow, $0.1 \mathrm{~mL} / \mathrm{min}$; Collision energy, $20 \mathrm{eV}$.

UV-Vis Spectroscopy. A $3 \mathrm{~mL}$ quartz cuvette was equilibrated at $37^{\circ} \mathrm{C}$ in a Cary 100 spectrophotometer with $2.46 \mathrm{~mL}$ of one of three solvent systems (A, PBS; B, 1:1 MeOH:PBS; C, 2:1 MeCN:PBS) for 5 mins and then baselined. Stock solutions of the complexes were prepared in DMSO at $1 \mathrm{mM}$, vortexed thoroughly, then sonicated for at least 10 mins at $37^{\circ} \mathrm{C}$ and visually inspected to ensure full dissolution. A solution of the complex ( $40 \mu \mathrm{L}, 16 \mu \mathrm{M}$ final) was then added to the cuvette and mixed by inversion 10 times before recording an initial spectrum. An aliquot of freshly prepared ascorbic acid solution ( $10 \mu \mathrm{L}$ of $16 \mathrm{mM}$ in cold PBS, $64 \mu \mathrm{M}$ final) was then added, and the cuvette mixed by inversion. Additional spectra were recorded at 2 and 10 minutes postaddition of ascorbic acid. Solvent systems were chosen to maintain solubility of the given complex with buffer at $37^{\circ} \mathrm{C}$. 


\section{Supplemental THF/PBD-BODIPY Co-autoxidation Data}

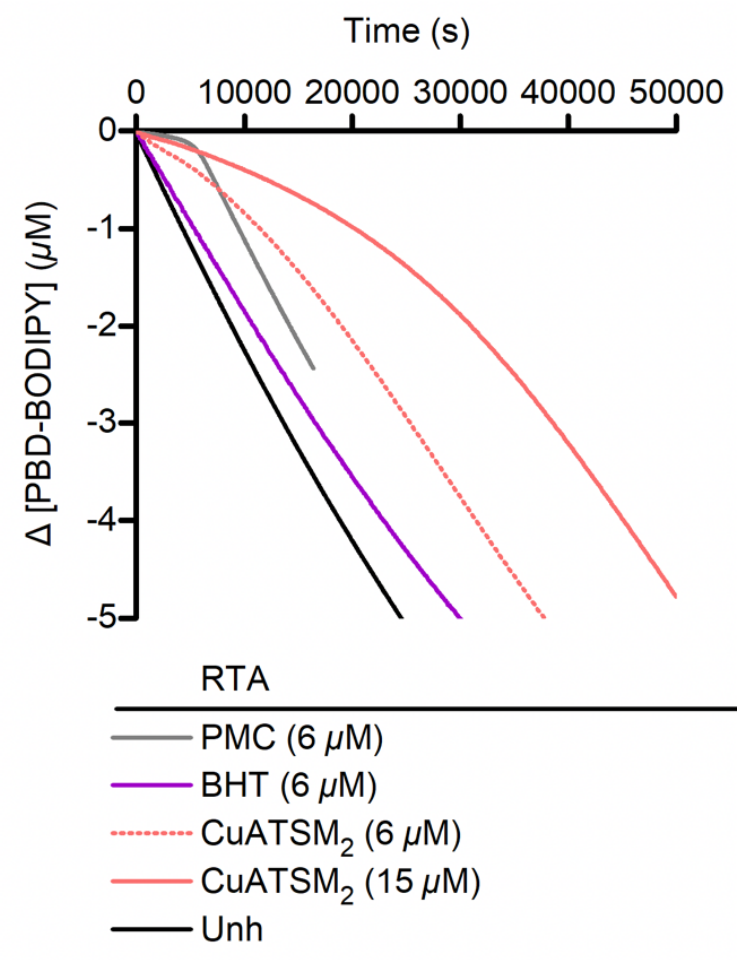

Figure S2. Uncropped representative traces of co-autoxidations of THF: $\mathrm{PhCl}(1: 3)$ and $\mathrm{PBD}-$ BODIPY $(10 \mu \mathrm{M})$ initiated with AIBN $(6 \mathrm{mM})$ at $37^{\circ} \mathrm{C}$ and inhibited by $\mathrm{CuATSM}_{2}, \mathrm{PMC}$, or 2,6-di-tert-butyl-4-methylphenol (BHT) at the indicated concentrations. Full conditions reported in manuscript Figure 1. 


\section{Supplemental Egg PC/STY-BODIPY Co-autoxidation Data}

A

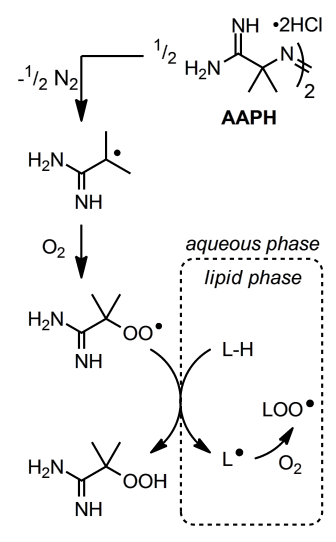

B

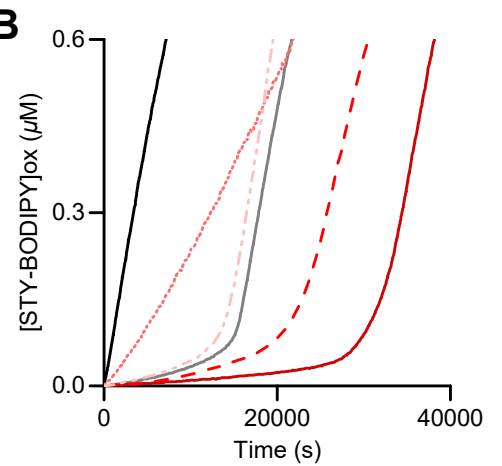

\begin{tabular}{|c|c|c|c|c|c|}
\hline $\mathrm{RTA}(4 \mu \mathrm{M})$ & $k_{\text {inh }}\left(\times 10^{4} \mathrm{M}^{-1} \mathrm{~s}^{-1}\right)$ & $n$ & RTA $(4 \mu \mathrm{M})$ & $k_{\mathrm{inh}}\left(\times 10^{4} \mathrm{M}^{-1} \mathrm{~s}^{-1}\right)$ & $n$ \\
\hline CUATSM & $11 \pm 1.3$ & $4.0 \pm 0.1$ & $-\mathrm{NiATSM}$ & $5.1 \pm 0.30$ & $2.8 \pm 0.1$ \\
\hline - - CuGTSM & $7.7 \pm 0.53$ & $2.9 \pm 0.1$ & - - NiGTSM & $3.0 \pm 0.29$ & $3.0 \pm 0.1$ \\
\hline$\ldots \mathrm{CuATSM}_{2}{ }^{*}$ & $0.65 \pm 0.08$ & $>1.8 \pm 0.2$ & $\ldots . . . . \mathrm{NiATSM}_{2}$ & $6.4 \pm 0.56$ & $3.4 \pm 0.4$ \\
\hline$\ldots-$ CuATSP* & $3.9 \pm 0.14$ & $1.9 \pm 0.1$ & $\ldots$ NiATSP & $2.0 \pm 0.27$ & $1.4 \pm 0.1$ \\
\hline$-\mathrm{PMC}$ & $2.2 \pm 0.09$ & $2.0 \pm 0.1$ & —Uninhibited & & \\
\hline
\end{tabular}

Figure S3. Representative co-autoxidations of STY-BODIPY $(1 \mu \mathrm{M})$ and egg PC liposomes (1 $\mathrm{mM})$ in PBS initiated with AAPH $(\mathbf{A}, 1 \mathrm{mM})$ at $37^{\circ} \mathrm{C}$ and inhibited by copper and nickel bis(thiosemicarbazone) complexes (B,C). Full conditions reported in manuscript Figure 2. 


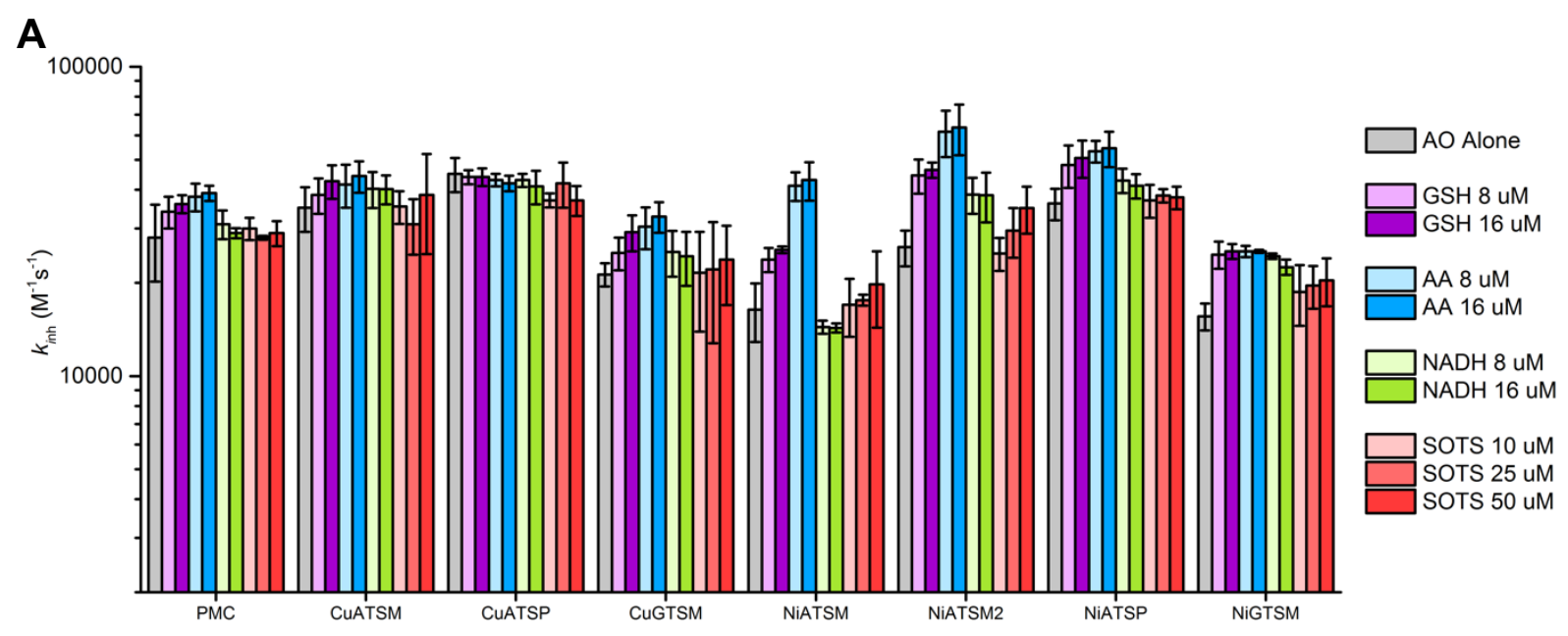

B
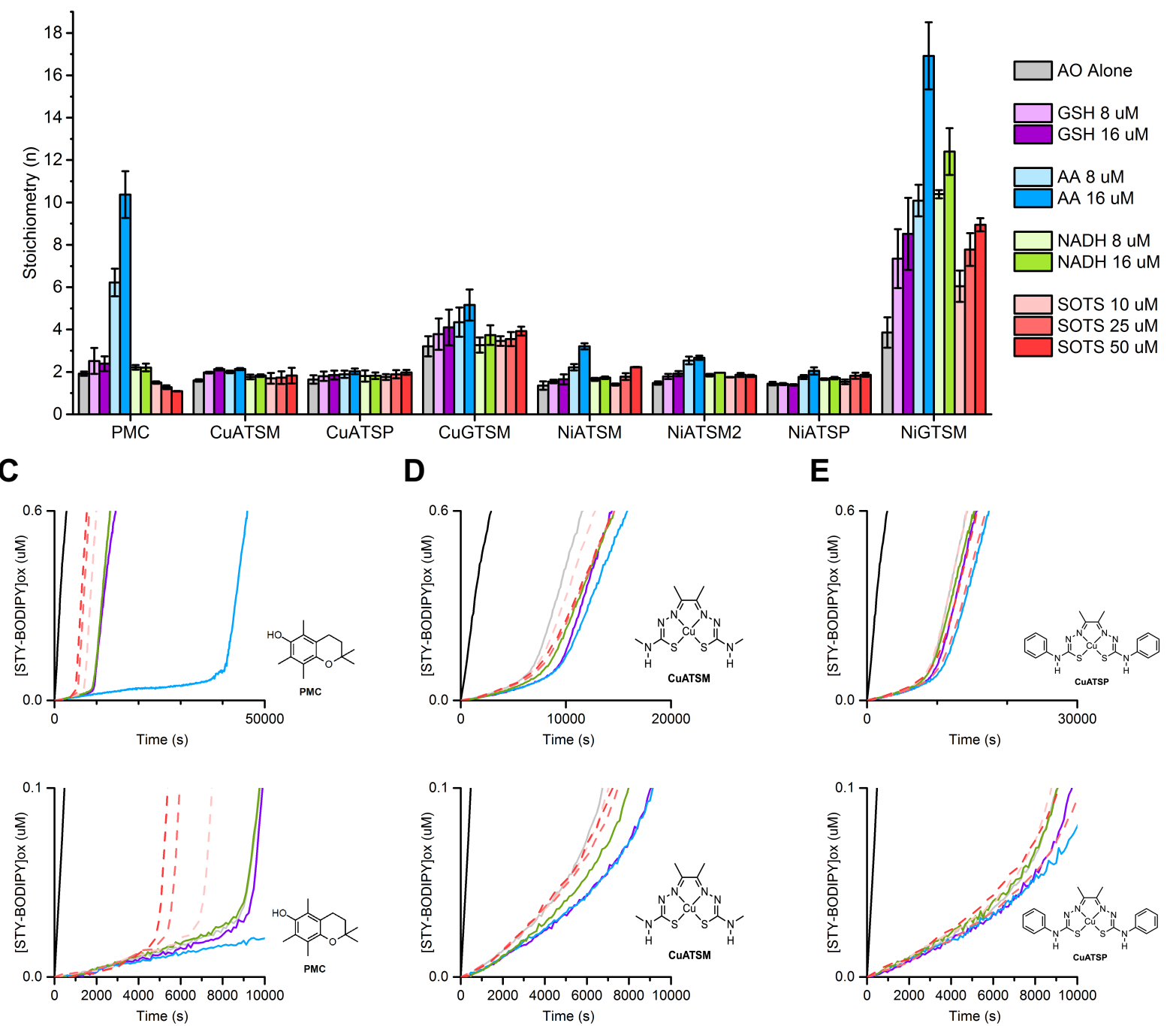
$\mathbf{F}$
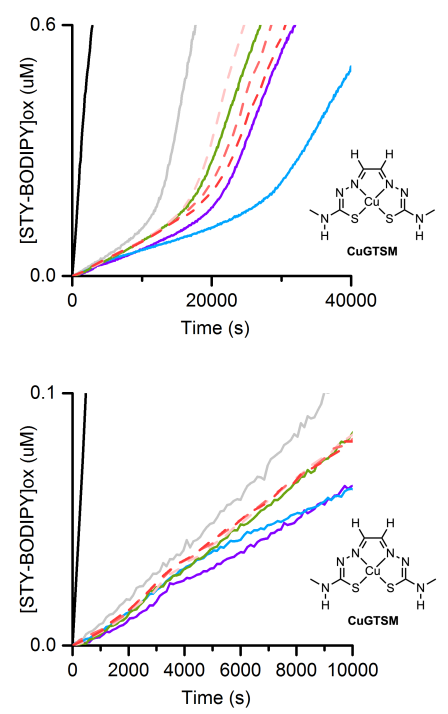

I
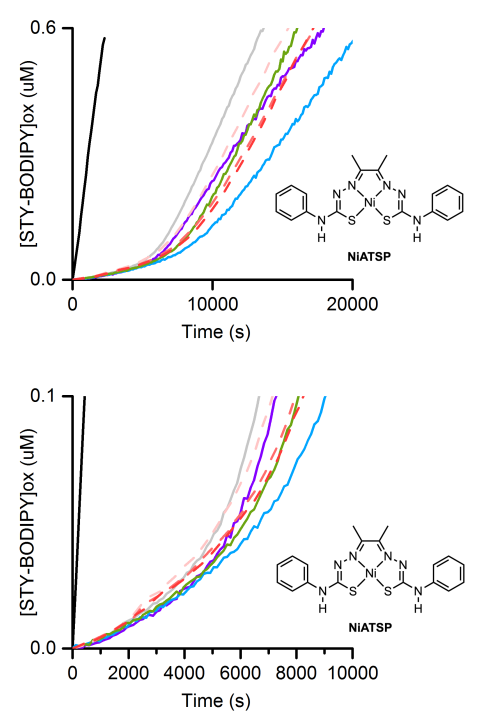

G
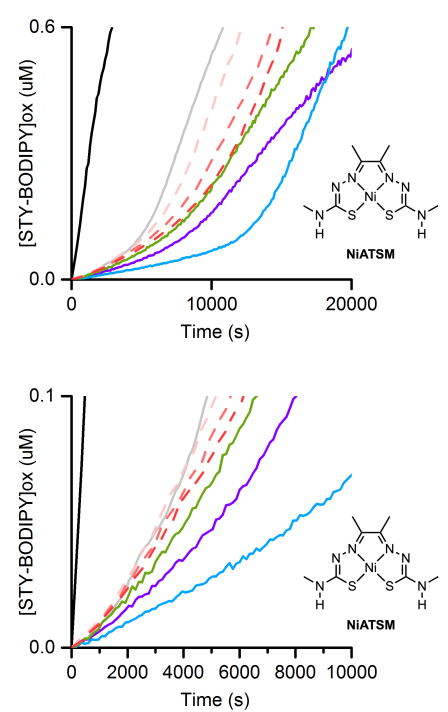

$\mathbf{J}$
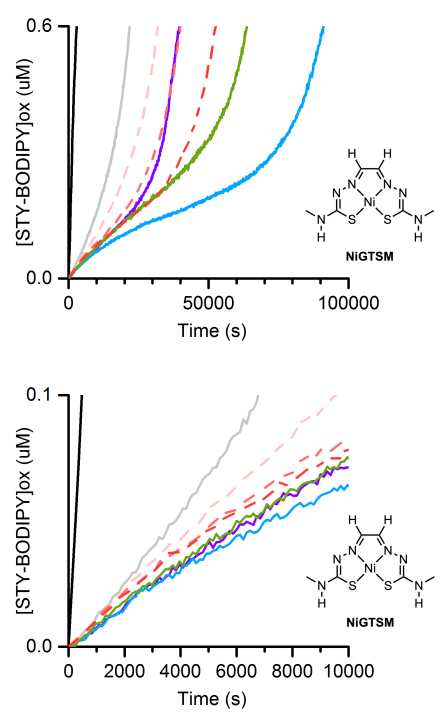

H
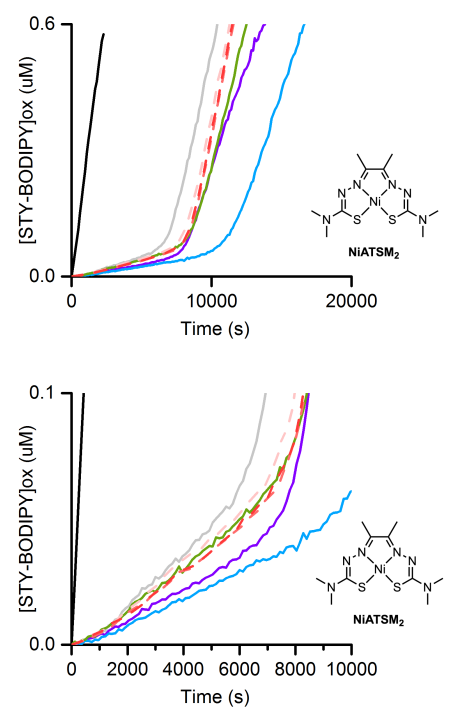

Figure S4. Summary of inhibition rate constants (A) and radical-trapping stoichiometry (B) obtained from co-autoxidations of STY-BODIPY $(1 \mu \mathrm{M})$ and egg PC $(1 \mathrm{mM})$ in PBS initiated with DTUN $(0.2 \mathrm{mM})$ at $37^{\circ} \mathrm{C}$ and inhibited by bis(thiosemicarbazone) copper and nickel complexes with and without reductants. Representative raw data are shown in panels $\mathbf{C}-\mathbf{J}$. The bottom graph of each panel are expansions of the initial portion of the reactions. Full conditions are reported in manuscript Figure 2. 
UV-Vis Spectral Data for Reduction of Metal Bis(Thiosemicarbazone) Complexes with Ascorbate
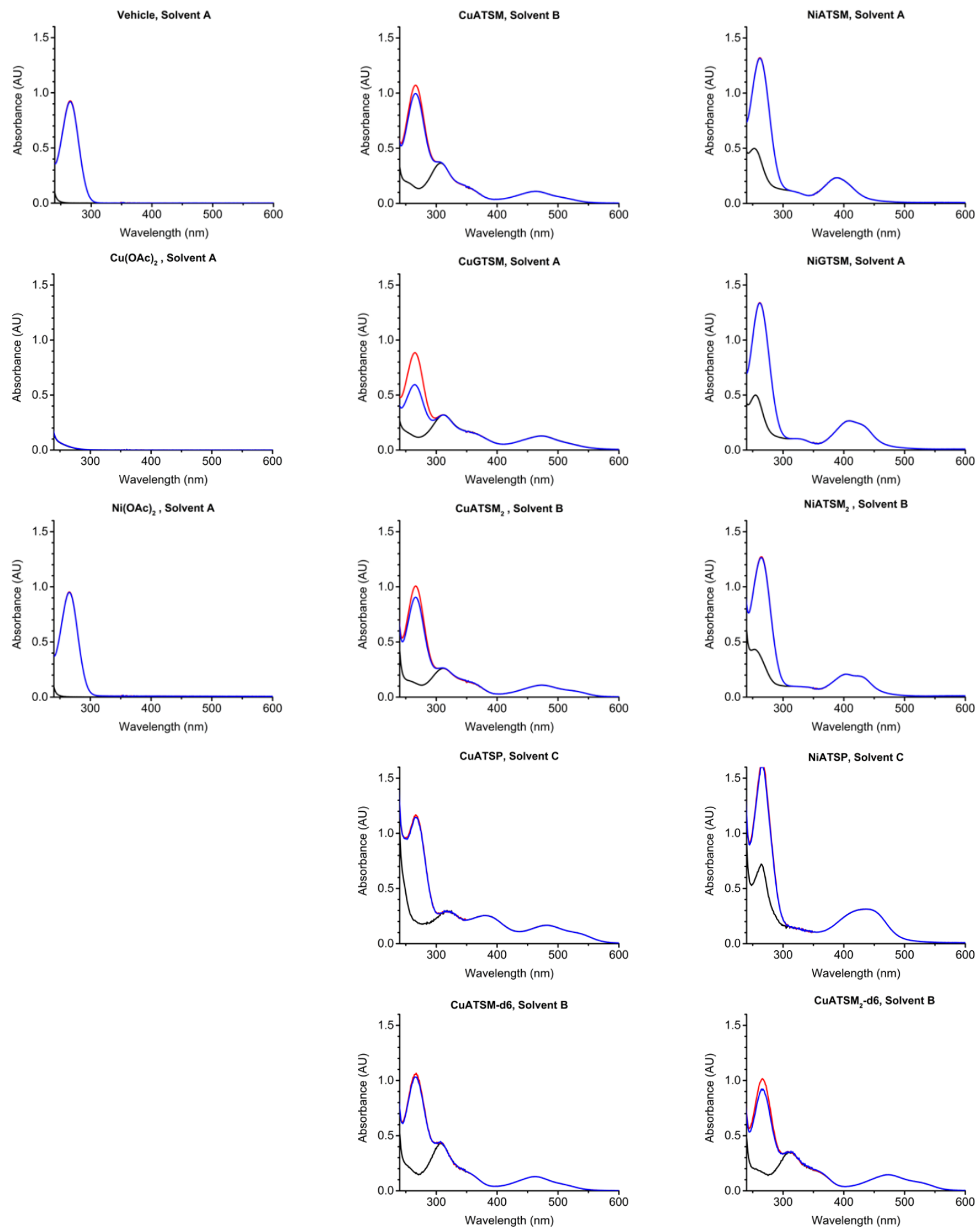

Figure S5. UV-Vis spectra of indicated complexes $(16 \mu \mathrm{M})$ in the indicated solvents with $1.6 \%$ DMSO recorded at $37^{\circ} \mathrm{C}$ (black) and with added ascorbic acid ( $64 \mu \mathrm{M}, 4$ eq; red, scan after 2 mins; blue, scan after 10 mins). 


\section{Ferroptosis Cell Rescue Assays in HT-22 Cells}
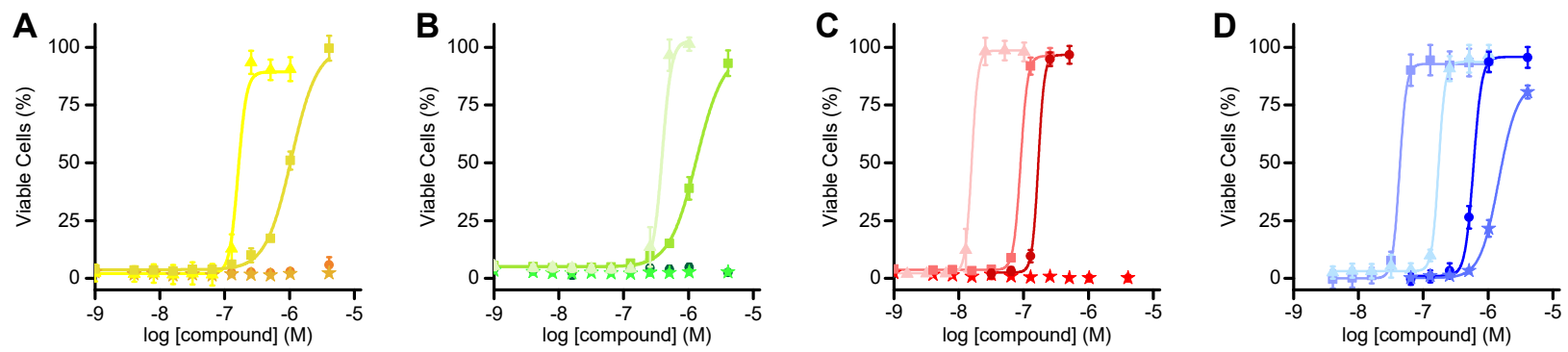

\begin{tabular}{|c|c|c|c|c|c|c|c|c|c|c|}
\hline & Compound & $\mathrm{EC}_{50}(\mathrm{nM})$ & & Compound & $\mathrm{EC}_{50}(\mathrm{nM})$ & & Compound & $\mathrm{EC}_{50}(\mathrm{nM})$ & Compound & $\mathrm{EC}_{50}(\mathrm{nM})$ \\
\hline 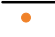 & $\mathrm{H}_{2}$-ATSM & n.e. & - & ZnATSM & n.e. & $\rightarrow$ & CuATSM & $170 \pm 5.3$ & $\because-$ NiATSM & $590 \pm 21$ \\
\hline$\star$ & $\mathrm{H}_{2}$-GTSM & n.e. & $\star$ & ZnGTSM & n.e. & $\star$ & CuGTSM & n.e. & \# NiGTSM & $1500 \pm 54$ \\
\hline- & $\mathrm{H}_{2}$-ATSM ${ }_{2}$ & $1100 \pm 81$ & $\rightarrow-$ & $\mathrm{ZnATSM}_{2}$ & $1300 \pm 89$ & $\rightarrow$ & CuATSM $_{2}$ & $90 \pm 3.3$ & $-\mathrm{NiATSM}_{2}$ & $43 \pm 5.2$ \\
\hline$=$ & $\mathrm{H}_{2}$-ATSP & $160 \pm 18$ & & ZnATSP & $390 \pm 26$ & $-\infty$ & CUATSP & $16 \pm 2.2$ & $=$ NiATSP & $170 \pm 16$ \\
\hline
\end{tabular}

Figure S6. (A-D) Rescue of RSL3-induced ferroptosis in HT22 cells (6000 cells in $100 \mu \mathrm{L} ; 150$ $\mathrm{nM}$ RSL3 for $5 \mathrm{~h}$ ) and associated $\mathrm{EC}_{50}$ values (n.e. = not effective). Supplemental to manuscript Figure 3. 


\section{UPLC Chromatograms of Copper Bis(thiosemicarbazone) Complexes.}

A

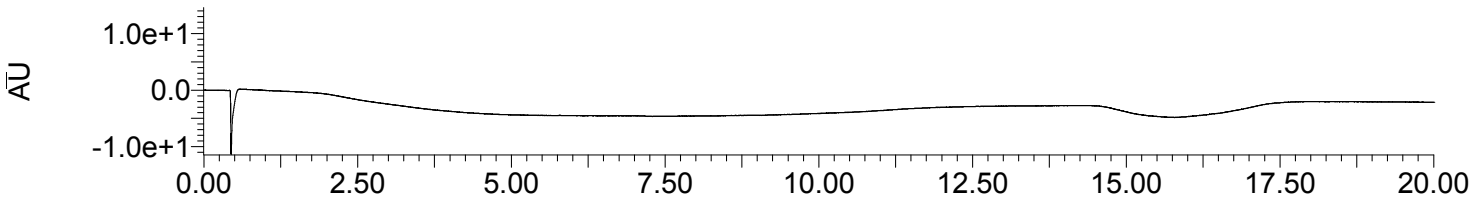

B

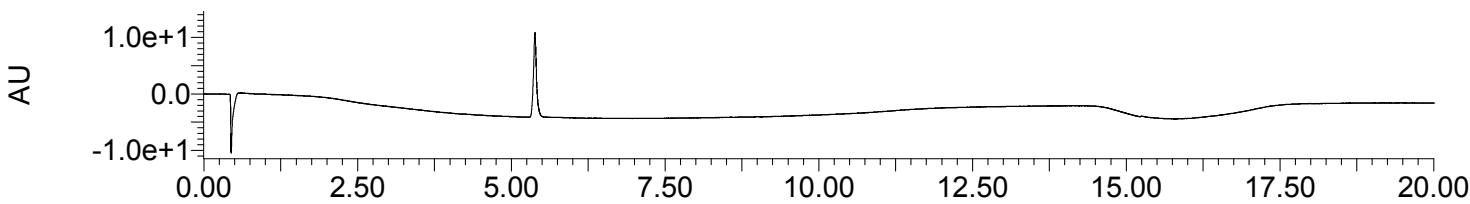

C

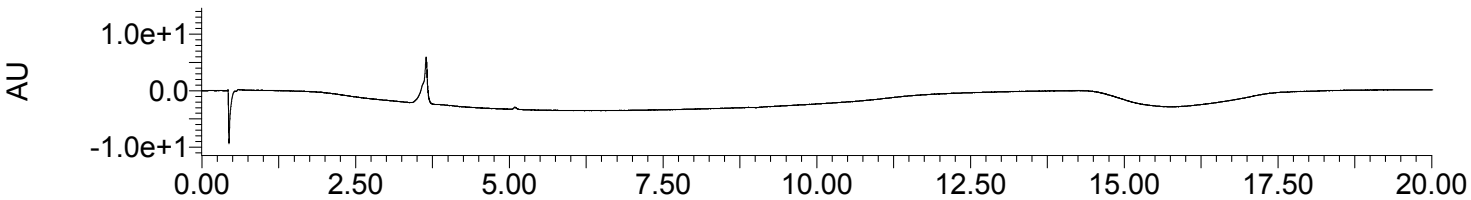

D

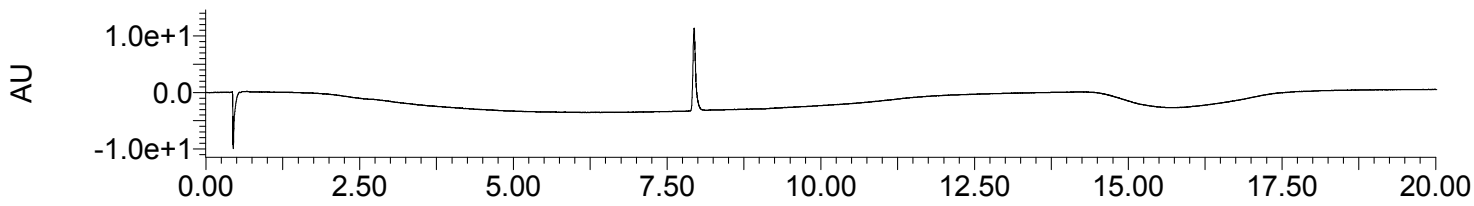

E

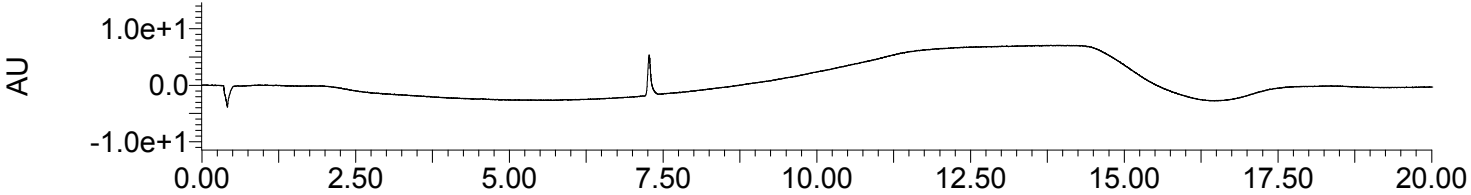

F

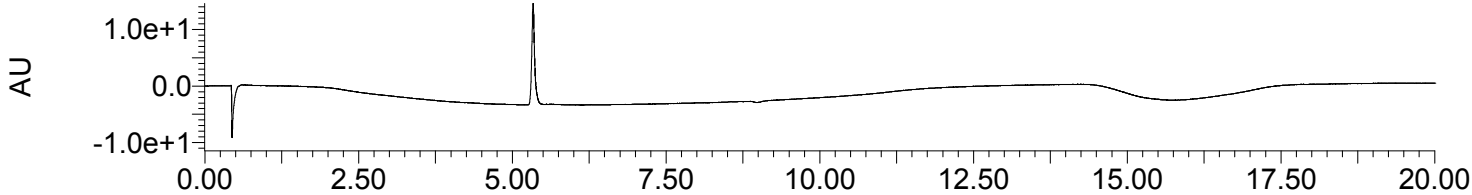

G

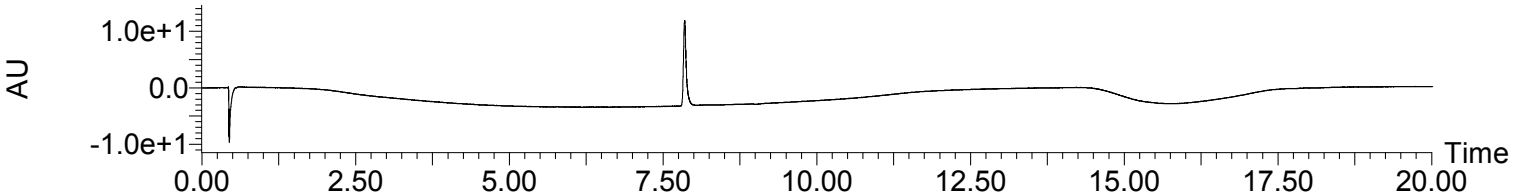

Figure S7. UPLC-PDA TIC chromatograms of $\mathrm{Cu}$ complexes $\left(10 \mu \mathrm{M}\right.$ in $\left.1: 1 \mathrm{MeCN}: \mathrm{H}_{2} \mathrm{O}\right)$ : (A) blank injection, (B) CuATSM, (C) CuGTSM, (D) $\mathrm{CuATSM}_{2}$, (E) CuATSP, (F) CuATSMd6, (G) $\mathrm{CuATSM}_{2}$-d6. For details on conditions, see general section above. 
NMR Spectra
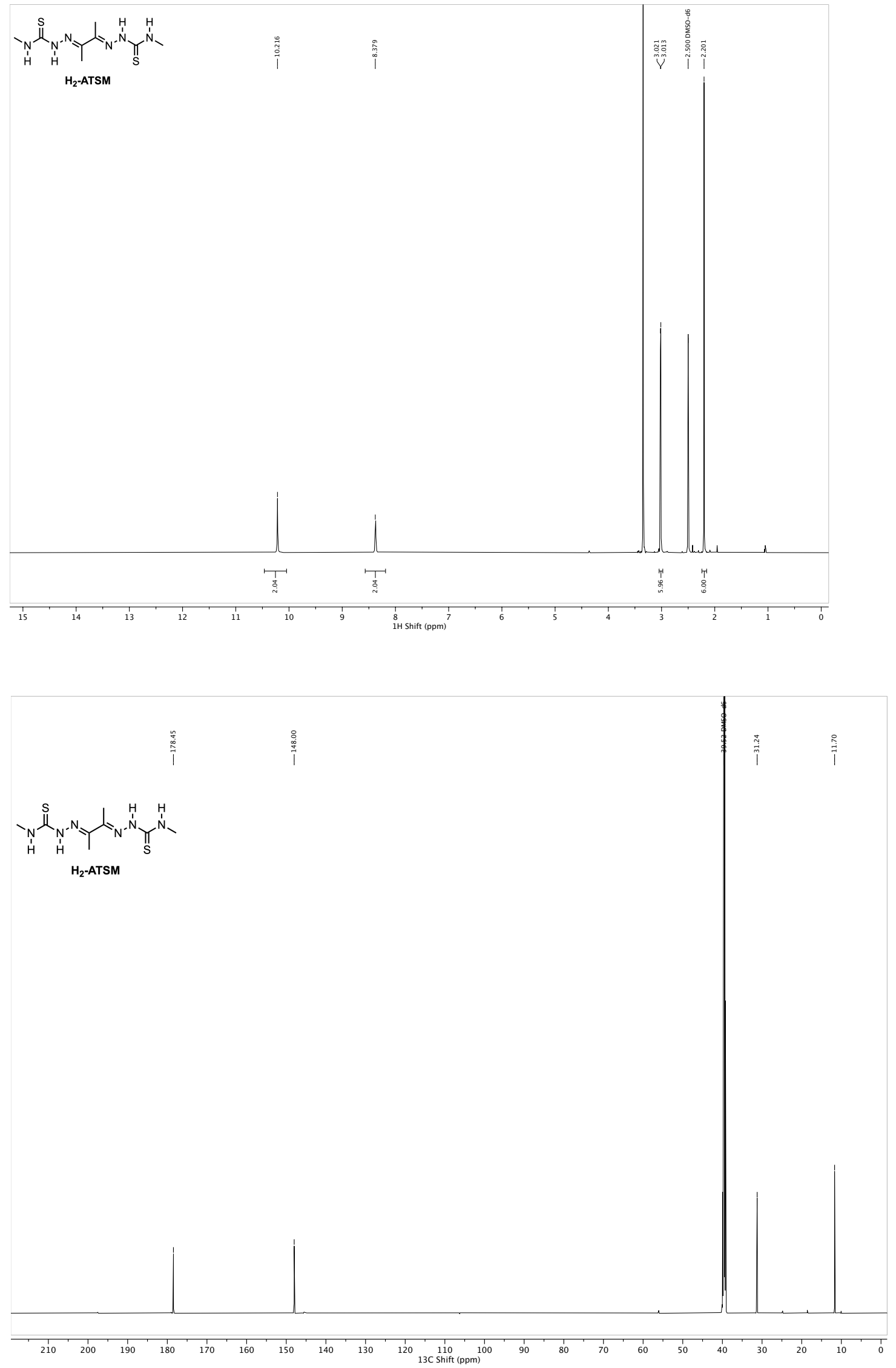

Figure S8. ${ }^{1} \mathrm{H}$ and ${ }^{13} \mathrm{C}$ NMR spectra of $\mathrm{H}_{2}$-ATSM in DMSO- $d_{6}$. 

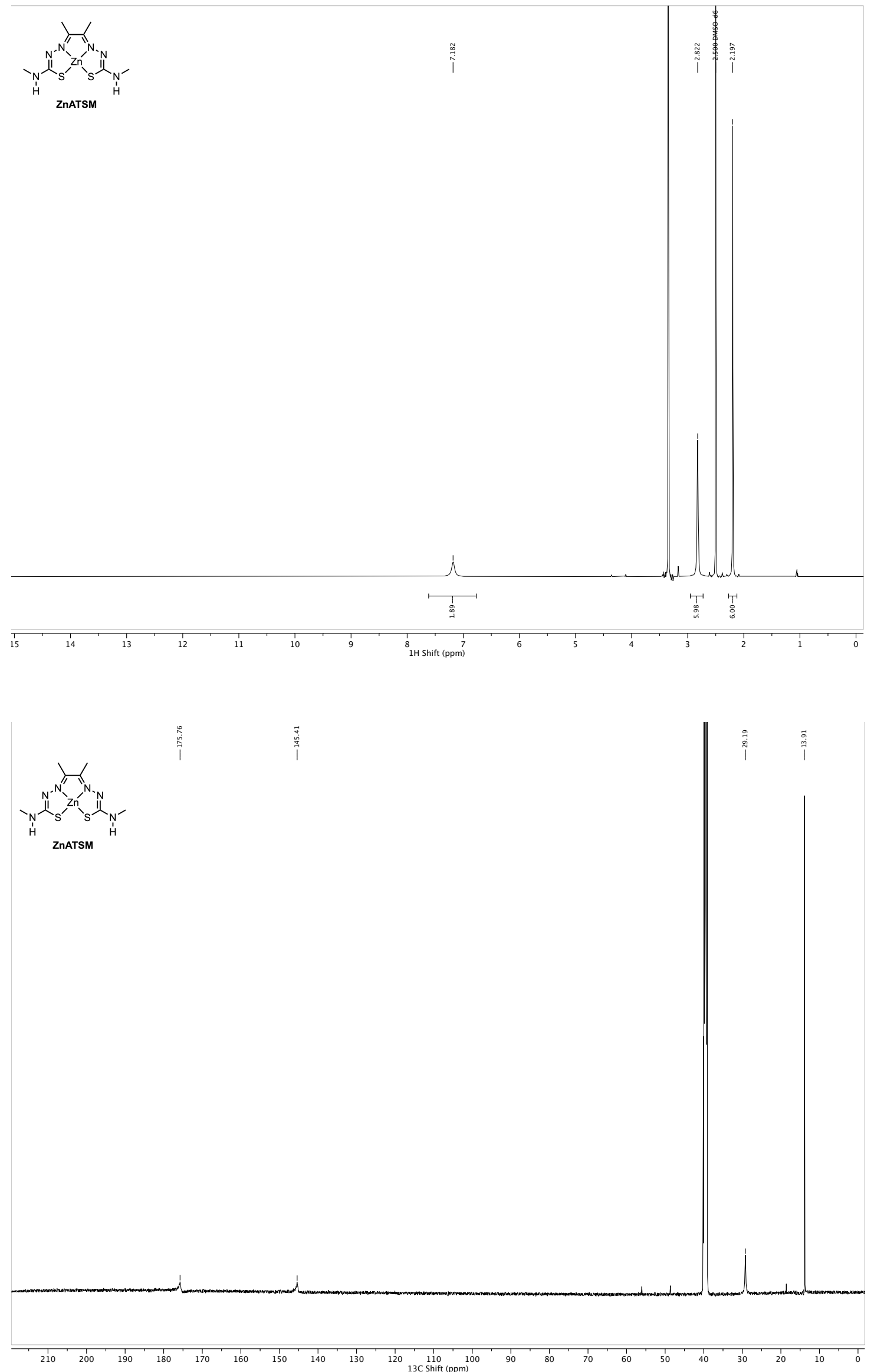

Figure S9. ${ }^{1} \mathrm{H}$ and ${ }^{13} \mathrm{C}$ NMR spectra of ZnATSM in DMSO- $d_{6}$. 

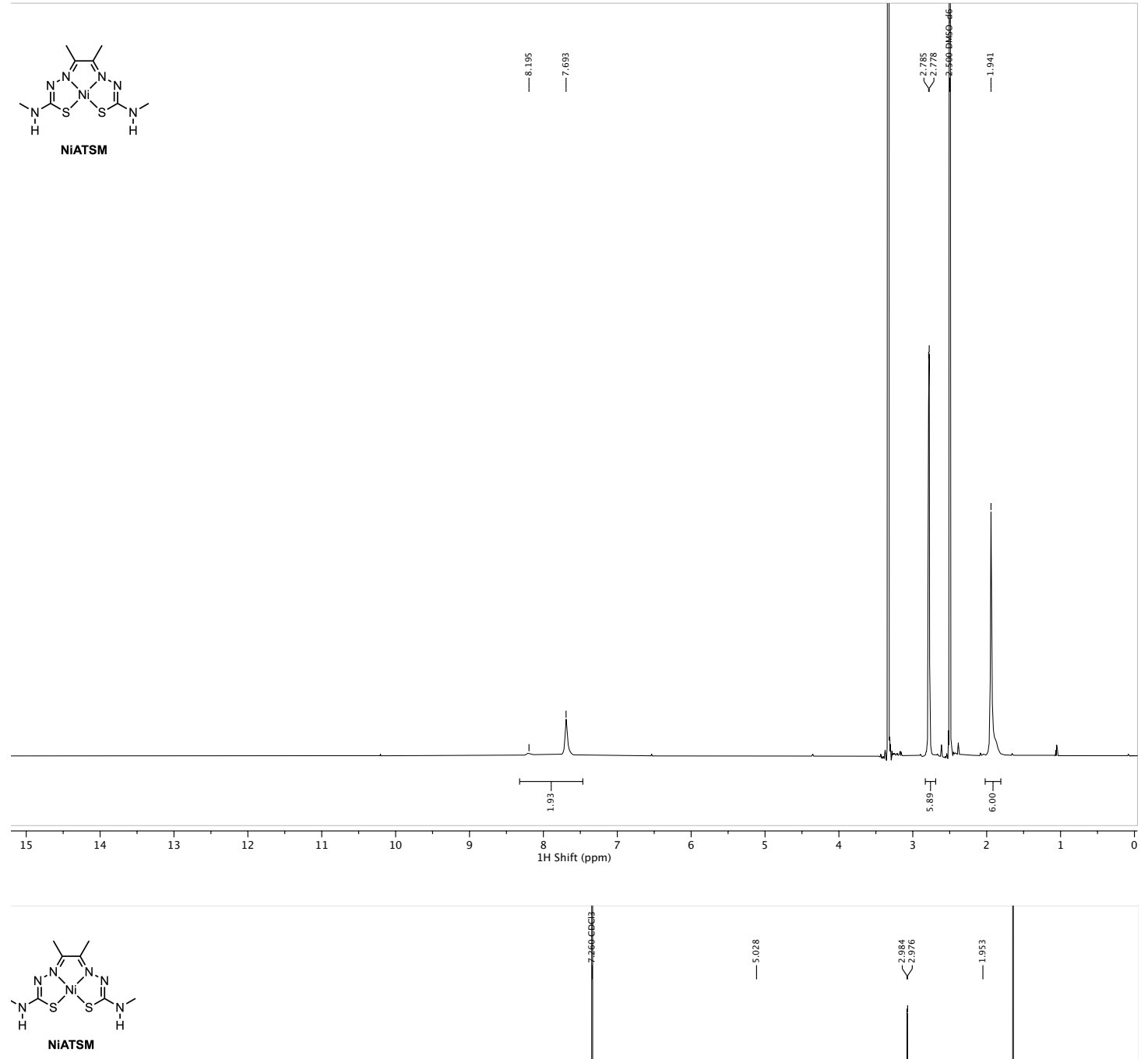

$\alpha_{2}^{\mathrm{H}}=0.0066+0.133\left(\delta_{D M S O}-\delta_{C D C l 3}\right)$

$\alpha_{2}^{\mathrm{H}}=0.0066+0.133(7.69-5.03)$

$\alpha_{2}^{\mathrm{H}}=0.36$

\begin{tabular}{l} 
\\
\\
\\
\\
\\
$.0066+0.133\left(\delta_{D M S O}-\delta_{C D C l 3}\right)$ \\
$.0066+0.133(7.69-5.03)$ \\
\hline
\end{tabular}

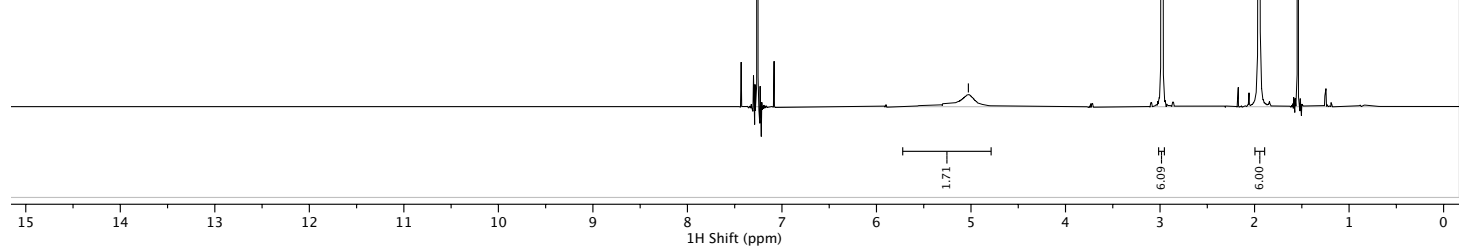

Figure S10. ${ }^{1} \mathrm{H}$ NMR spectrum of NiATSM in DMSO- $d_{6}$ and $\mathrm{CDCl}_{3}$ used to calculate $\alpha_{2}^{\mathrm{H}}$ (inset). 


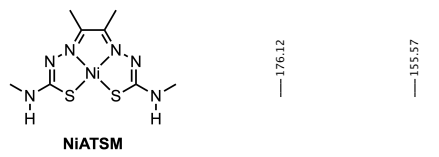

$\stackrel{\infty}{\tilde{N}} \stackrel{\mathscr{0}}{\stackrel{\infty}{\sim}}$

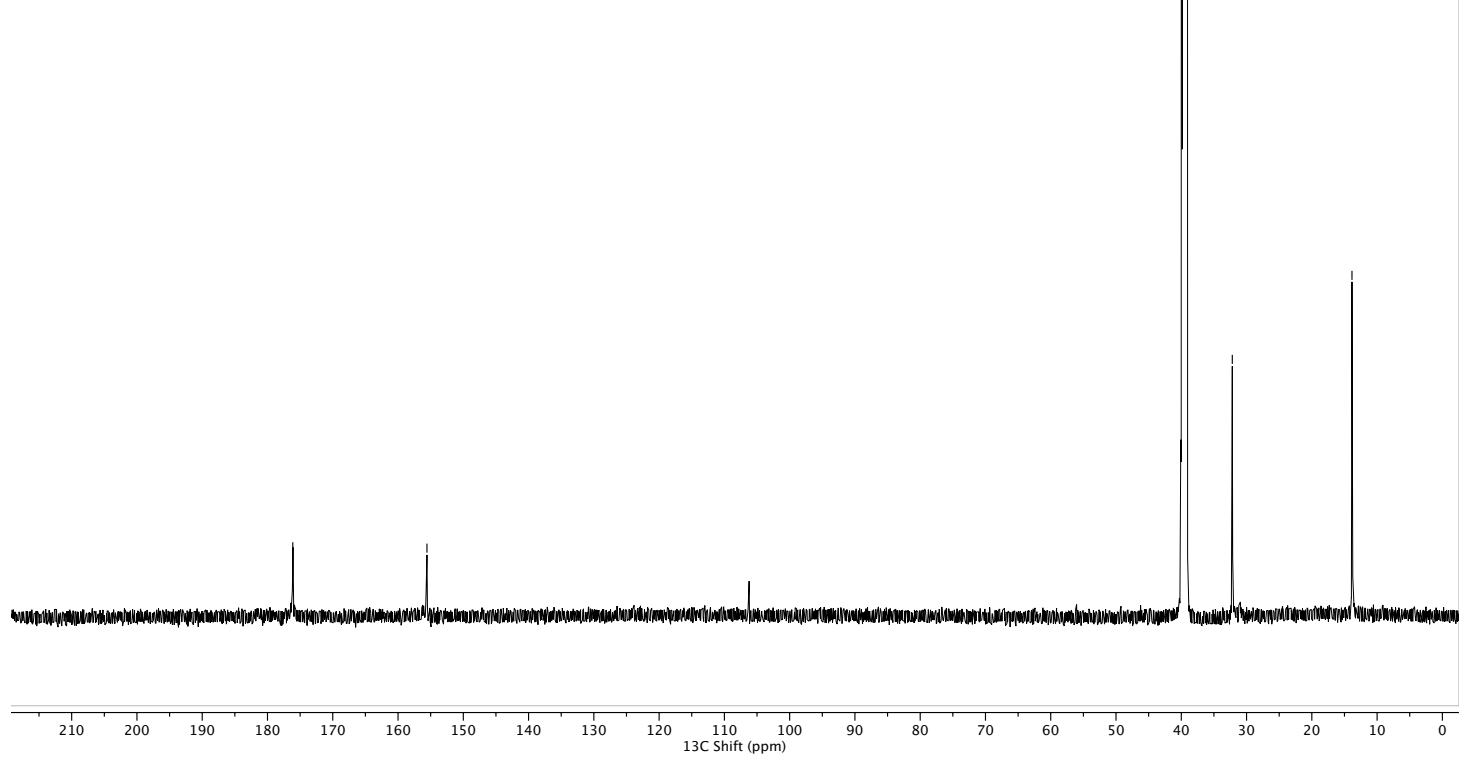

Figure S11. ${ }^{13} \mathrm{C}$ NMR spectrum of NiATSM in DMSO- $d_{6}$.
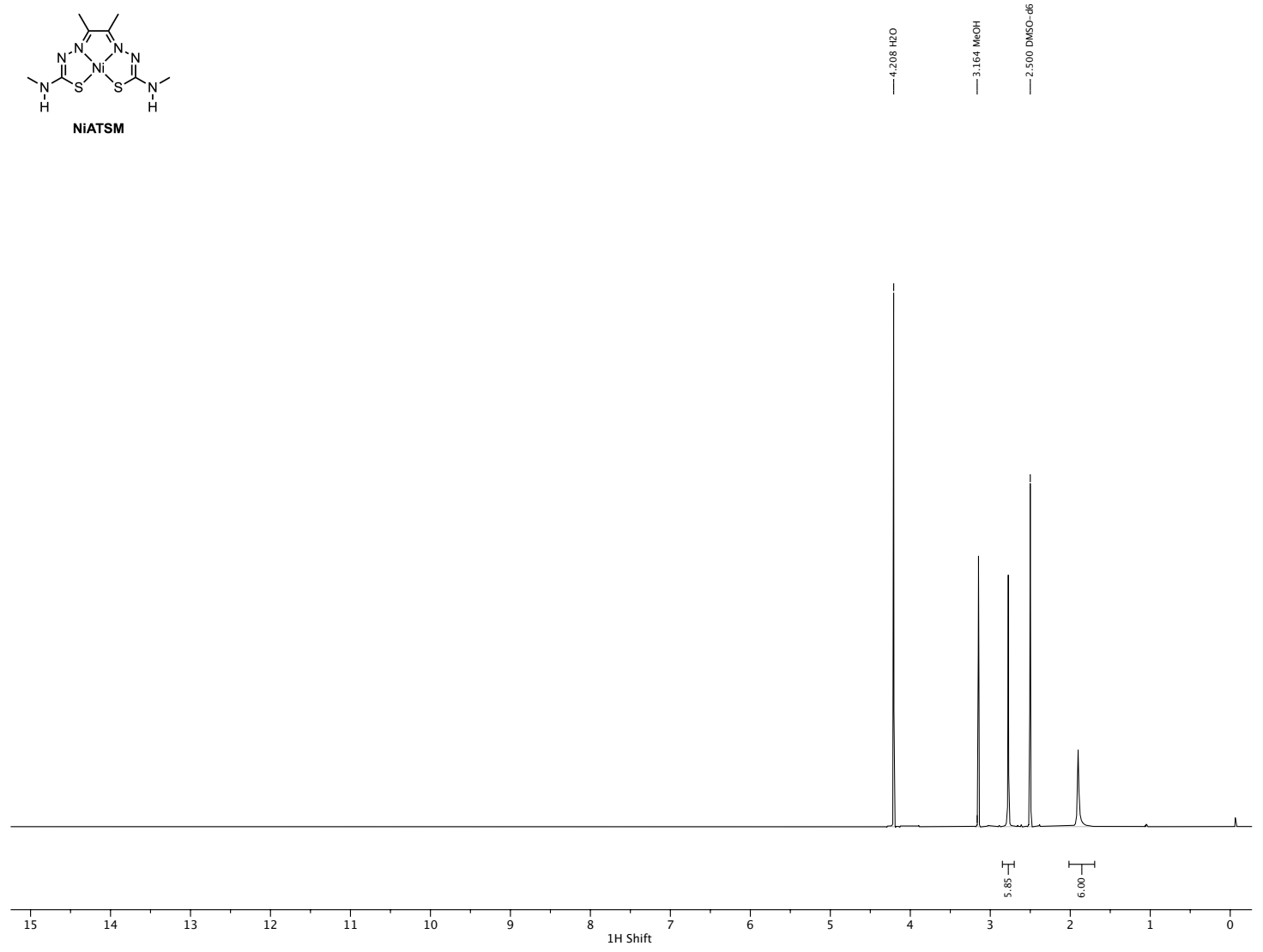

Figure S12. ${ }^{1} \mathrm{H}$ NMR spectrum of NiATSM in 1:1 DMSO- $d_{6}: \mathrm{MeOD}_{4}$ to verify H/D exchange. 

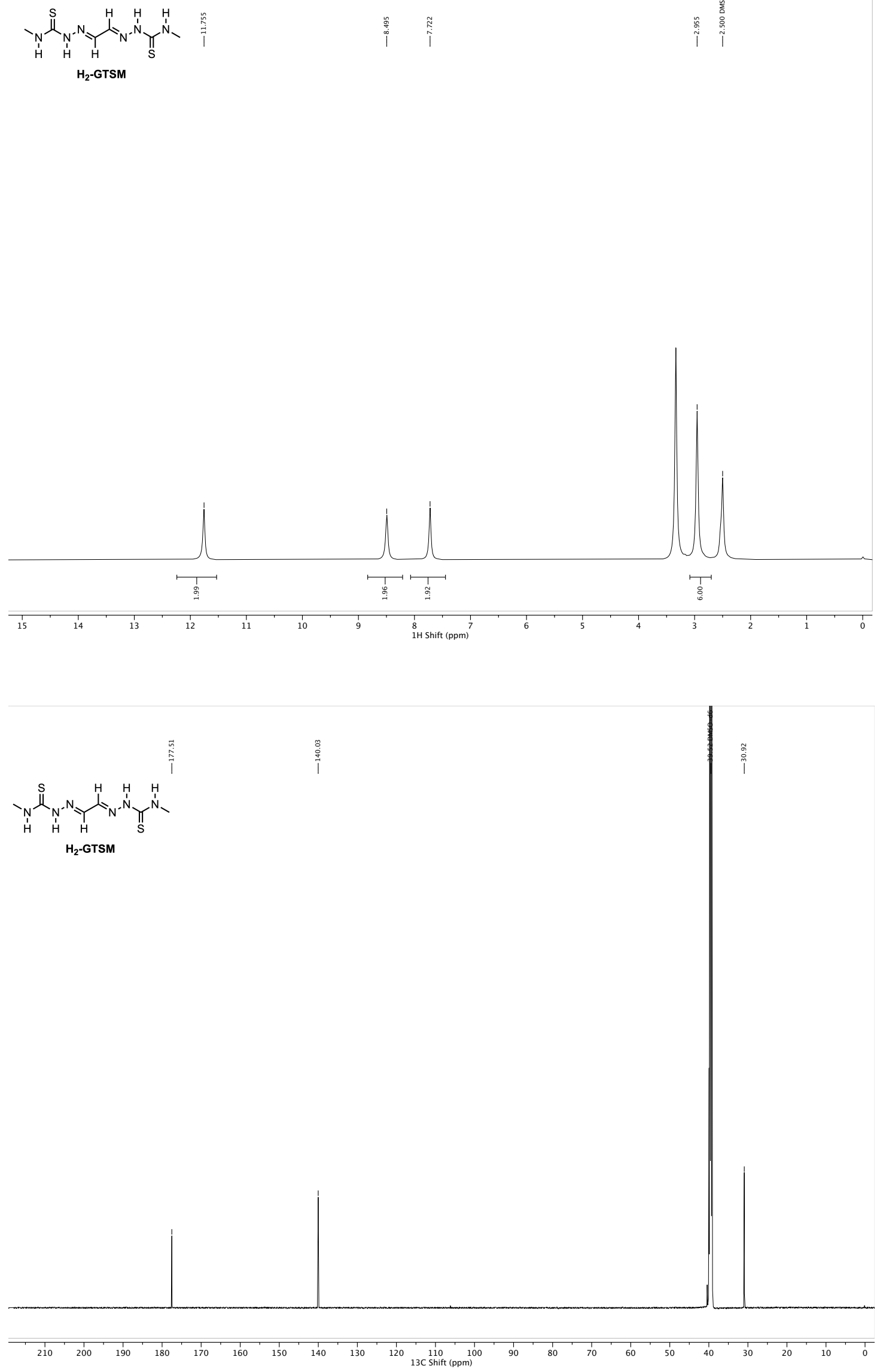

Figure S13. ${ }^{1} \mathrm{H}$ and ${ }^{13} \mathrm{C}$ NMR spectra of $\mathrm{H}_{2}-\mathrm{GTSM}$ in DMSO- $d_{6}$. 

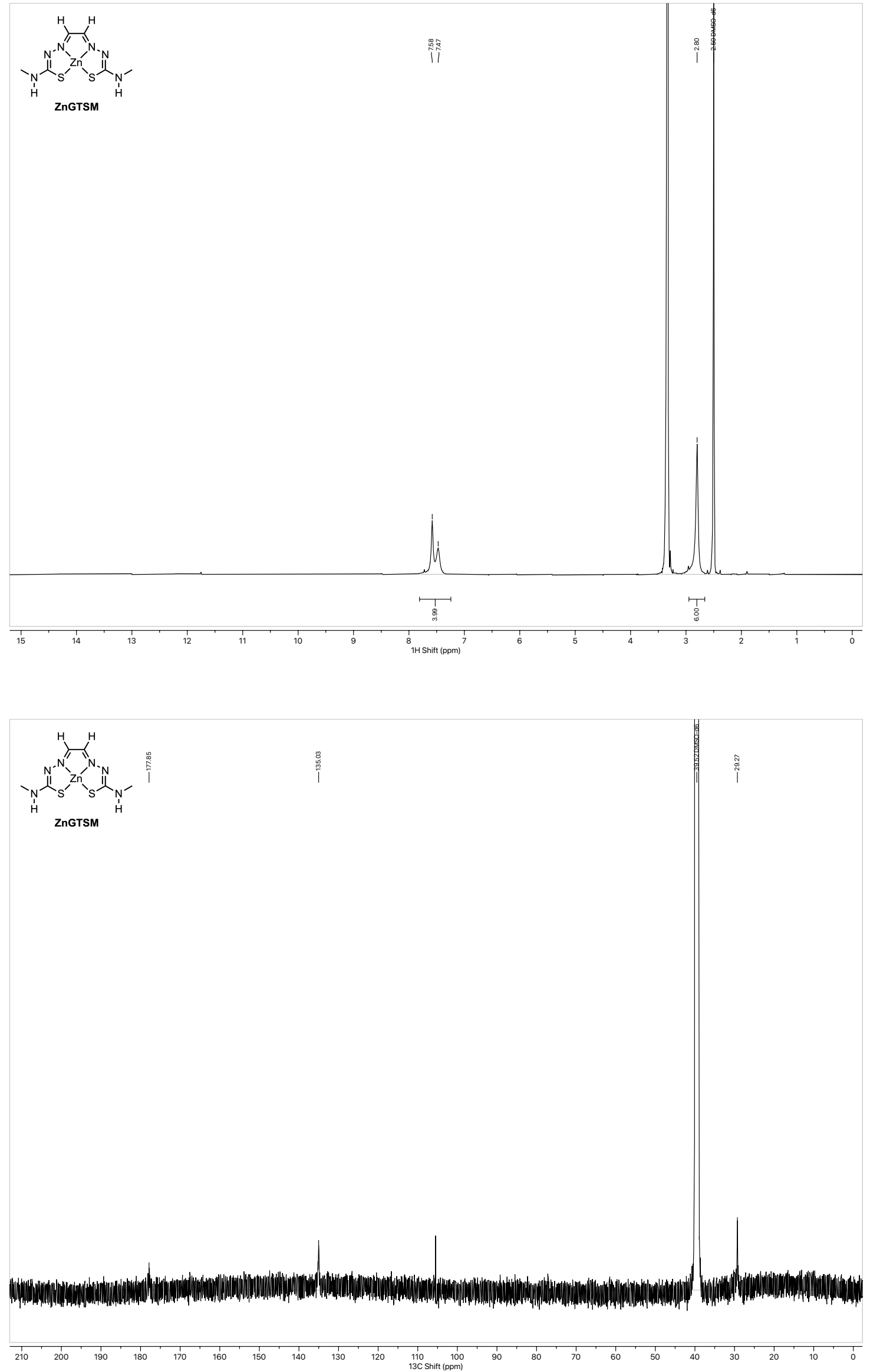

Figure S14. ${ }^{1} \mathrm{H}$ and ${ }^{13} \mathrm{C}$ NMR spectra of ZnGTSM in DMSO- $d_{6}$. 

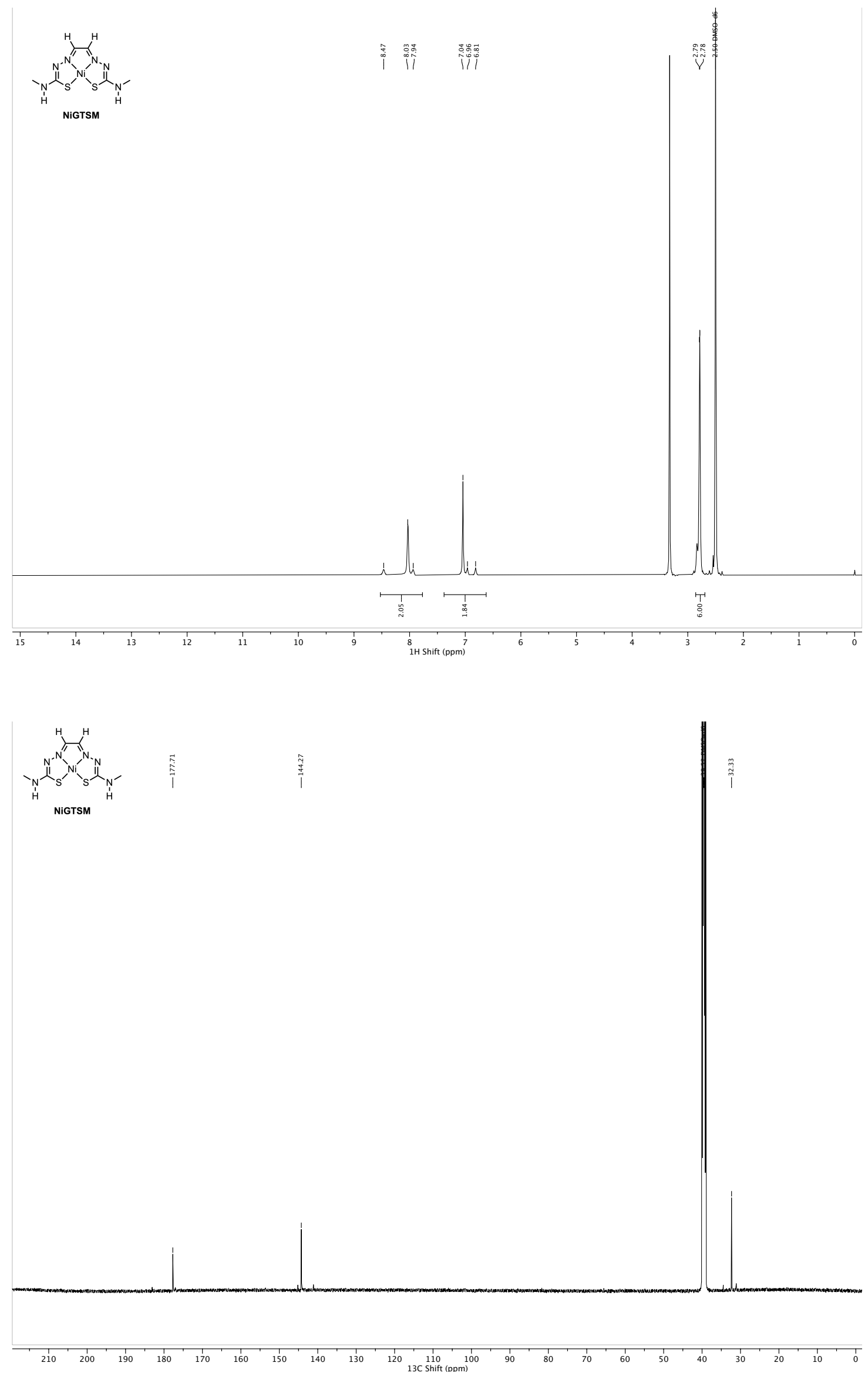

Figure S15. ${ }^{1} \mathrm{H}$ and ${ }^{13} \mathrm{C}$ NMR spectra of NiGTSM in DMSO- $d_{6}$. 

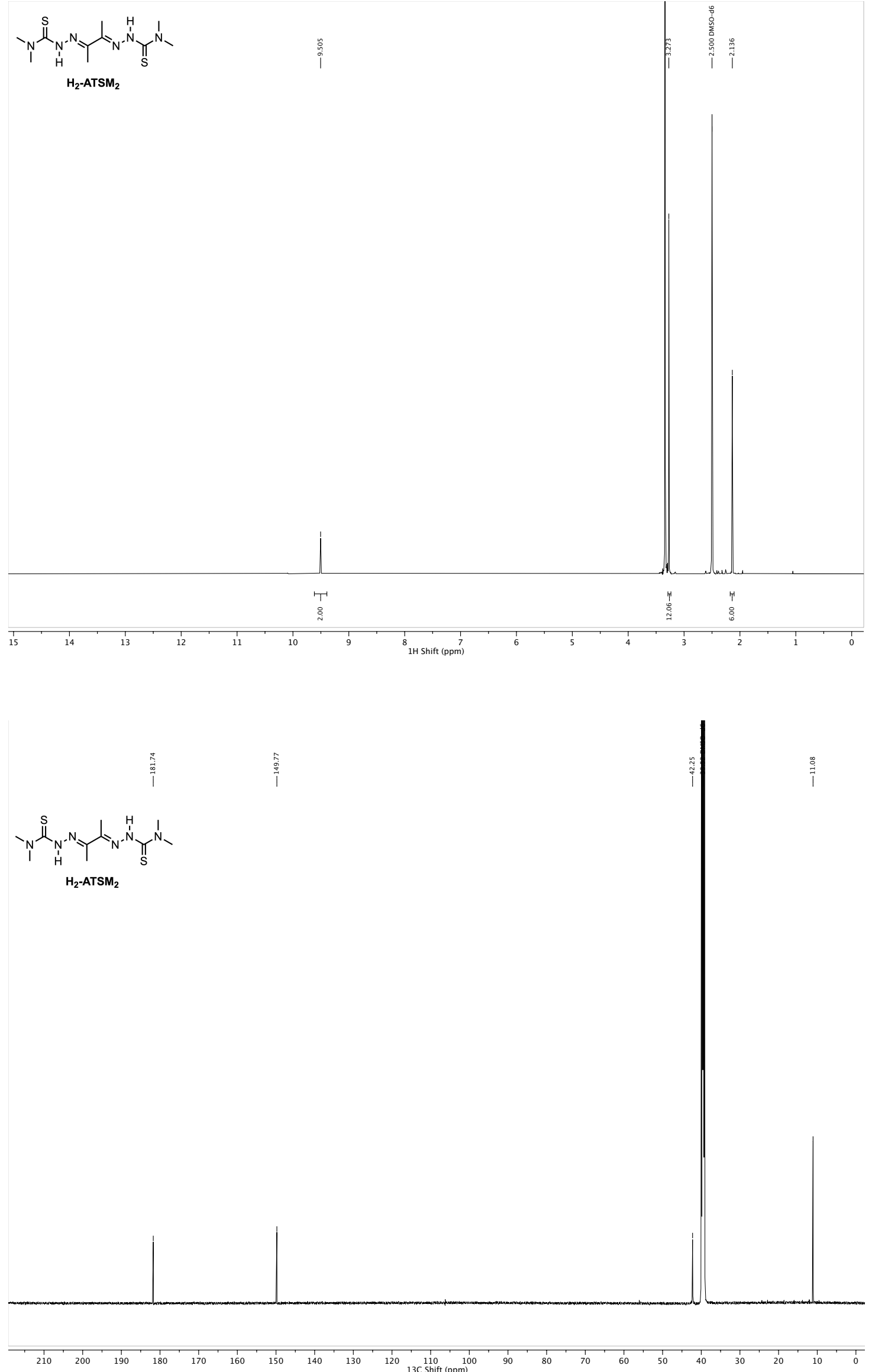

Figure S16. ${ }^{1} \mathrm{H}$ and ${ }^{13} \mathrm{C}$ NMR spectra of $\mathrm{H}_{2}-\mathrm{ATSM}_{2}$ in DMSO- $d_{6}$. 

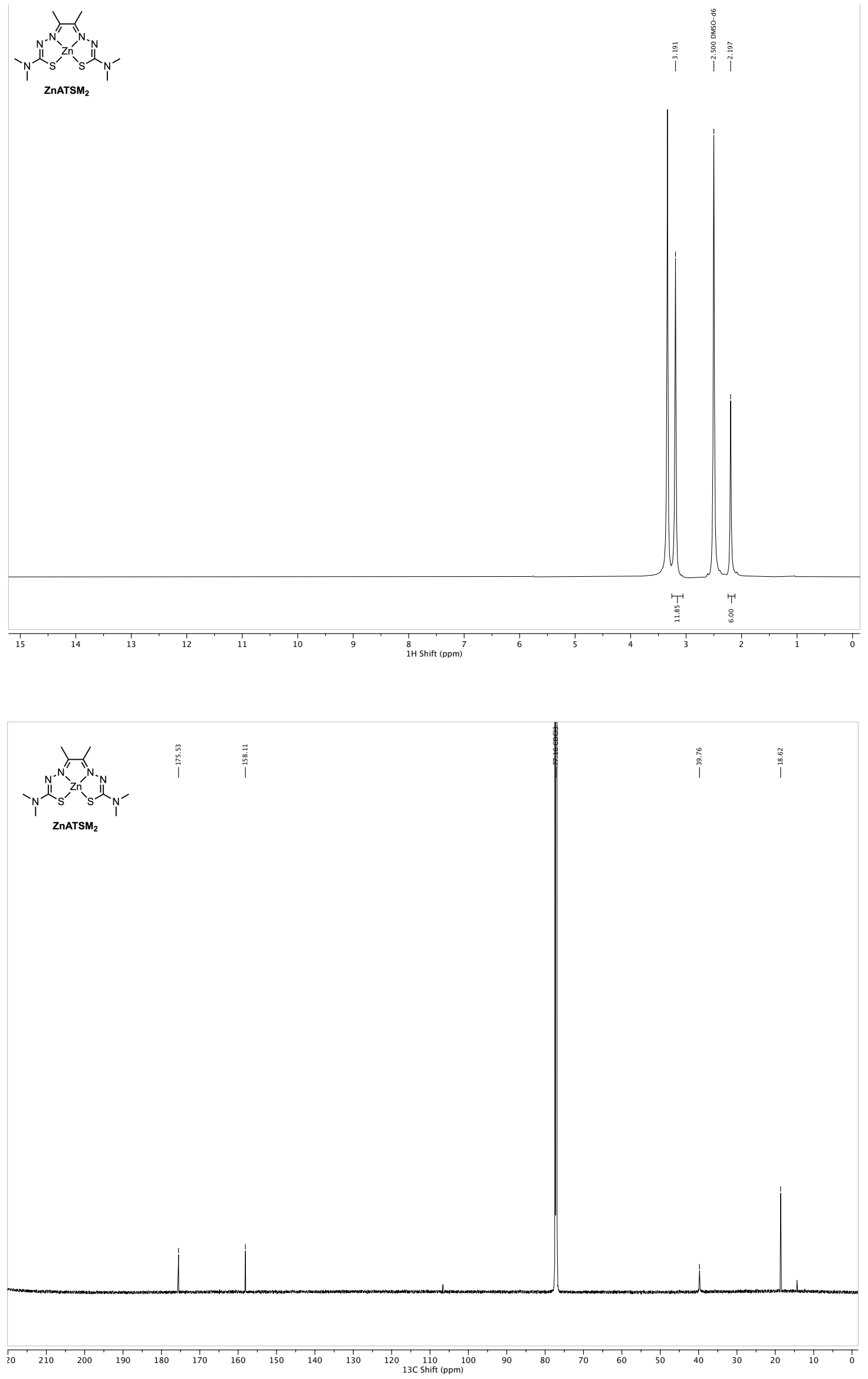

Figure S17. ${ }^{1} \mathrm{H}$ and ${ }^{13} \mathrm{C}$ NMR spectra of $\mathrm{ZnATSM}_{2}$ in DMSO- $d_{6}$. 

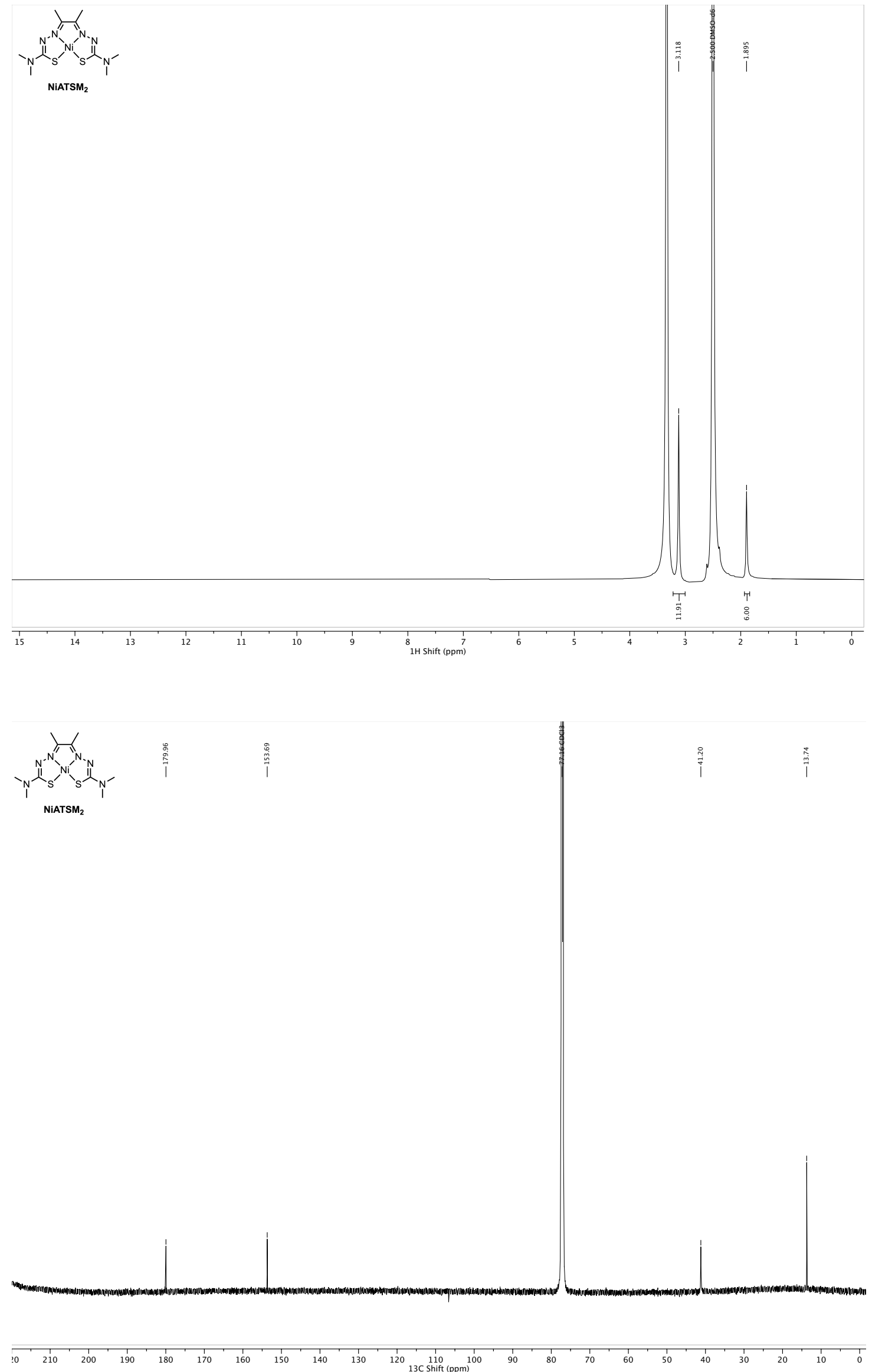

Figure S18. ${ }^{1} \mathrm{H}$ and ${ }^{13} \mathrm{C}$ NMR spectra of $\mathrm{NiATSM}_{2}$ in DMSO- $d_{6}$. 

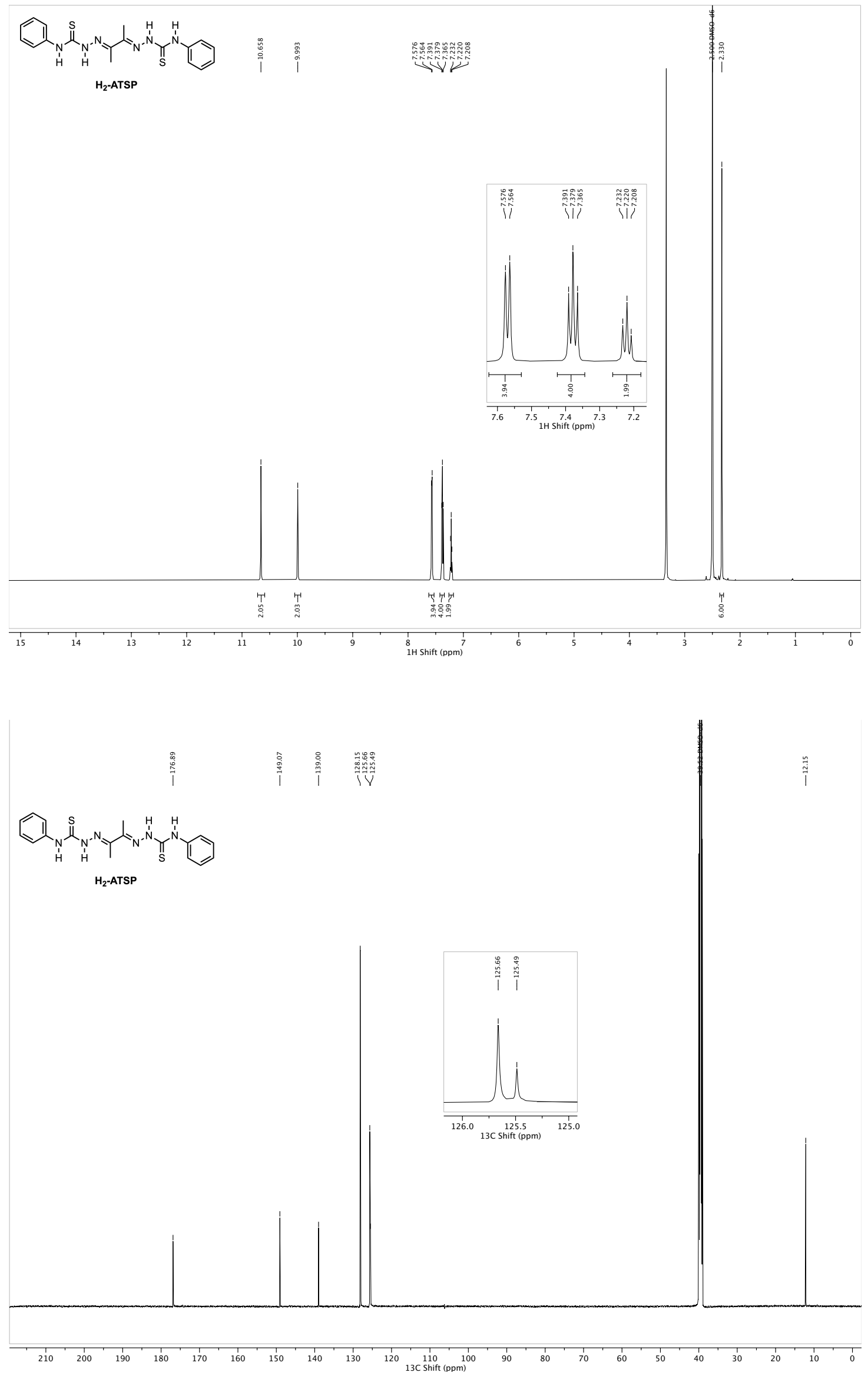

Figure S19. ${ }^{1} \mathrm{H}$ and ${ }^{13} \mathrm{C}$ NMR spectra of $\mathrm{H}_{2}$-ATSP in DMSO- $d_{6}$. 

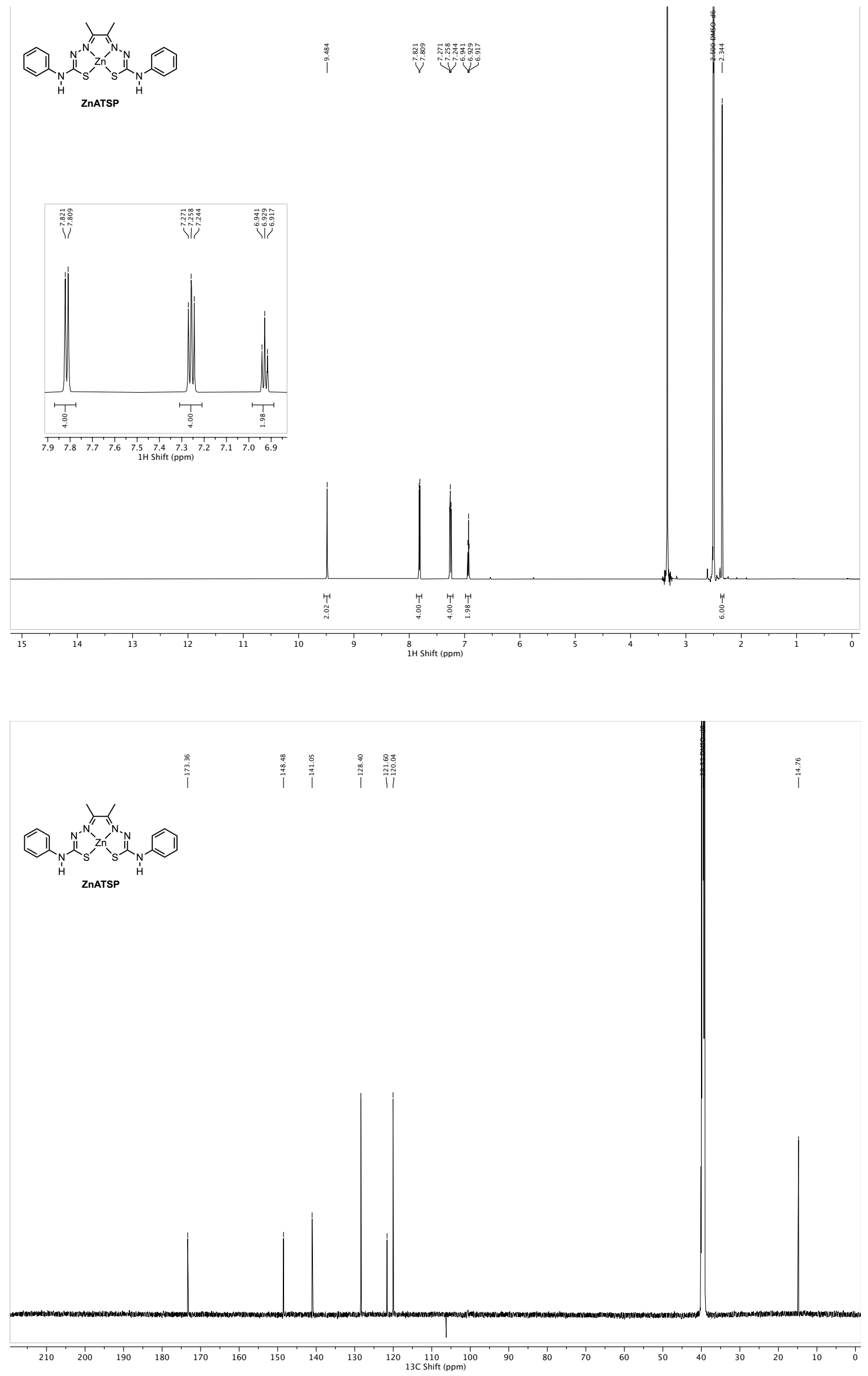

Figure S20. ${ }^{1} \mathrm{H}$ and ${ }^{13} \mathrm{C}$ NMR spectra of ZnATSP in DMSO- $d_{6}$. 

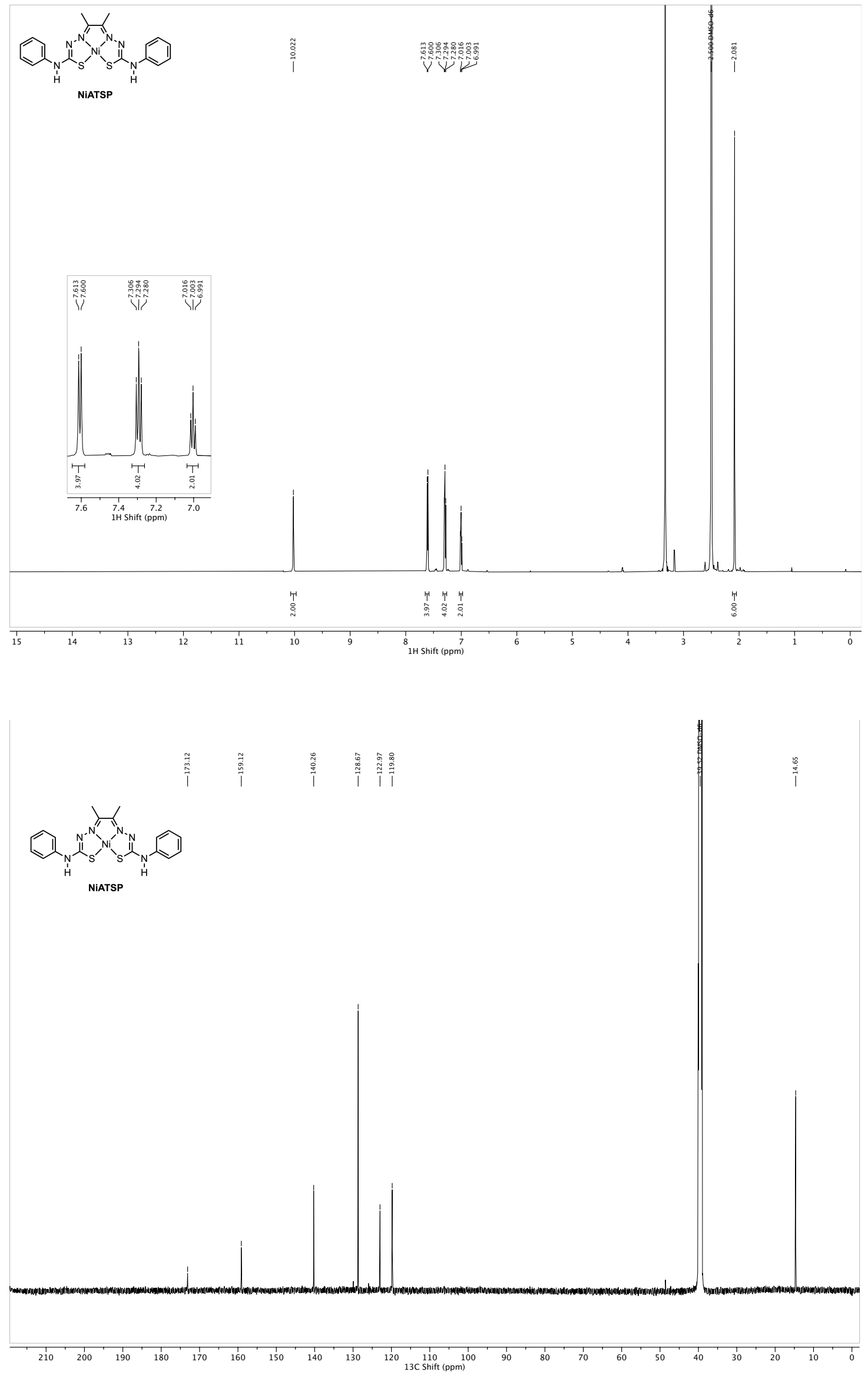

Figure S21. ${ }^{1} \mathrm{H}$ and ${ }^{13} \mathrm{C}$ NMR spectra of NiATSP in DMSO- $d_{6}$. 

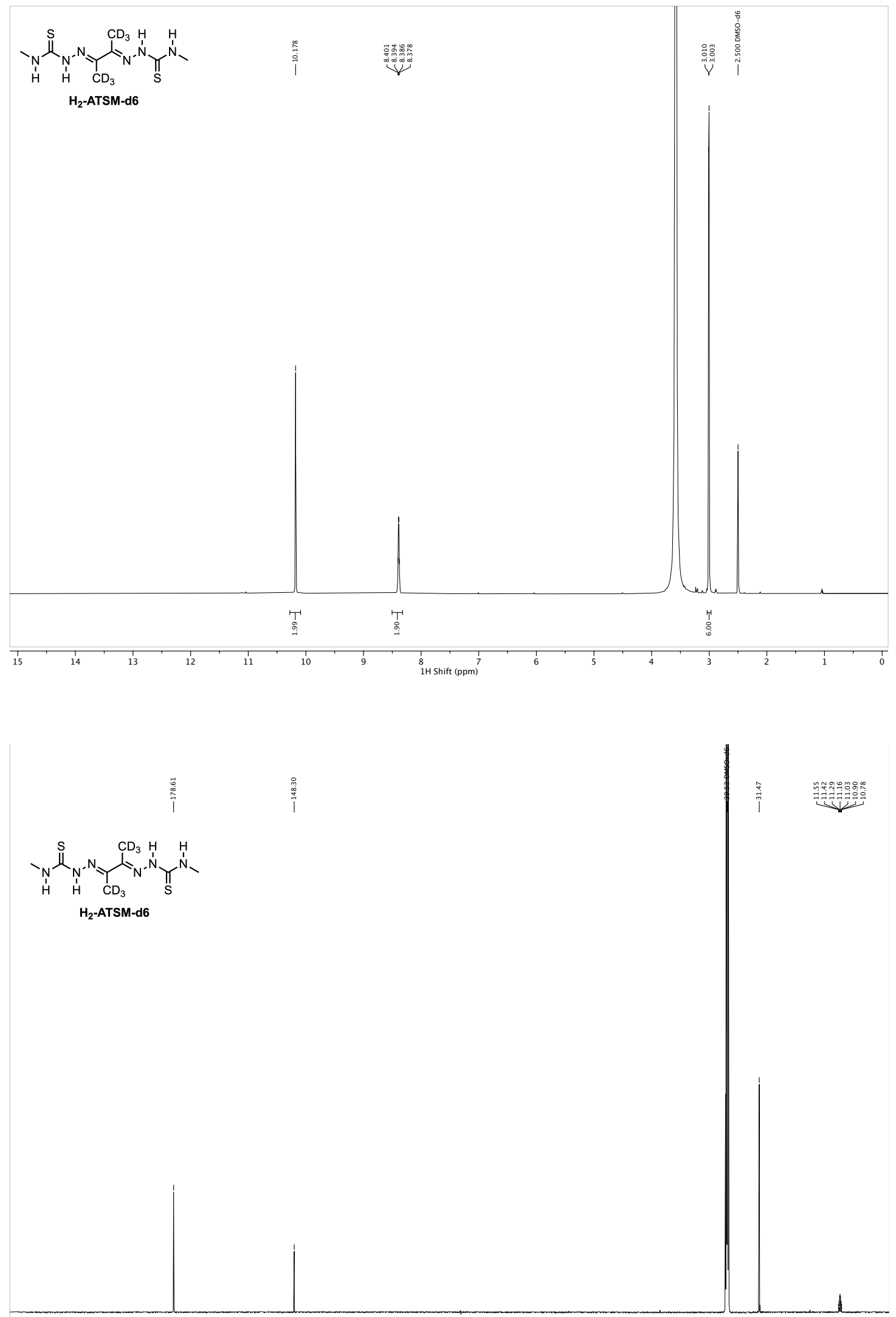

Figure S22. ${ }^{1} \mathrm{H}$ and ${ }^{13} \mathrm{C}$ NMR spectra of $\mathrm{H}_{2}-\mathrm{ATSM}-\mathrm{d} 6$ in DMSO- $d_{6}$. 

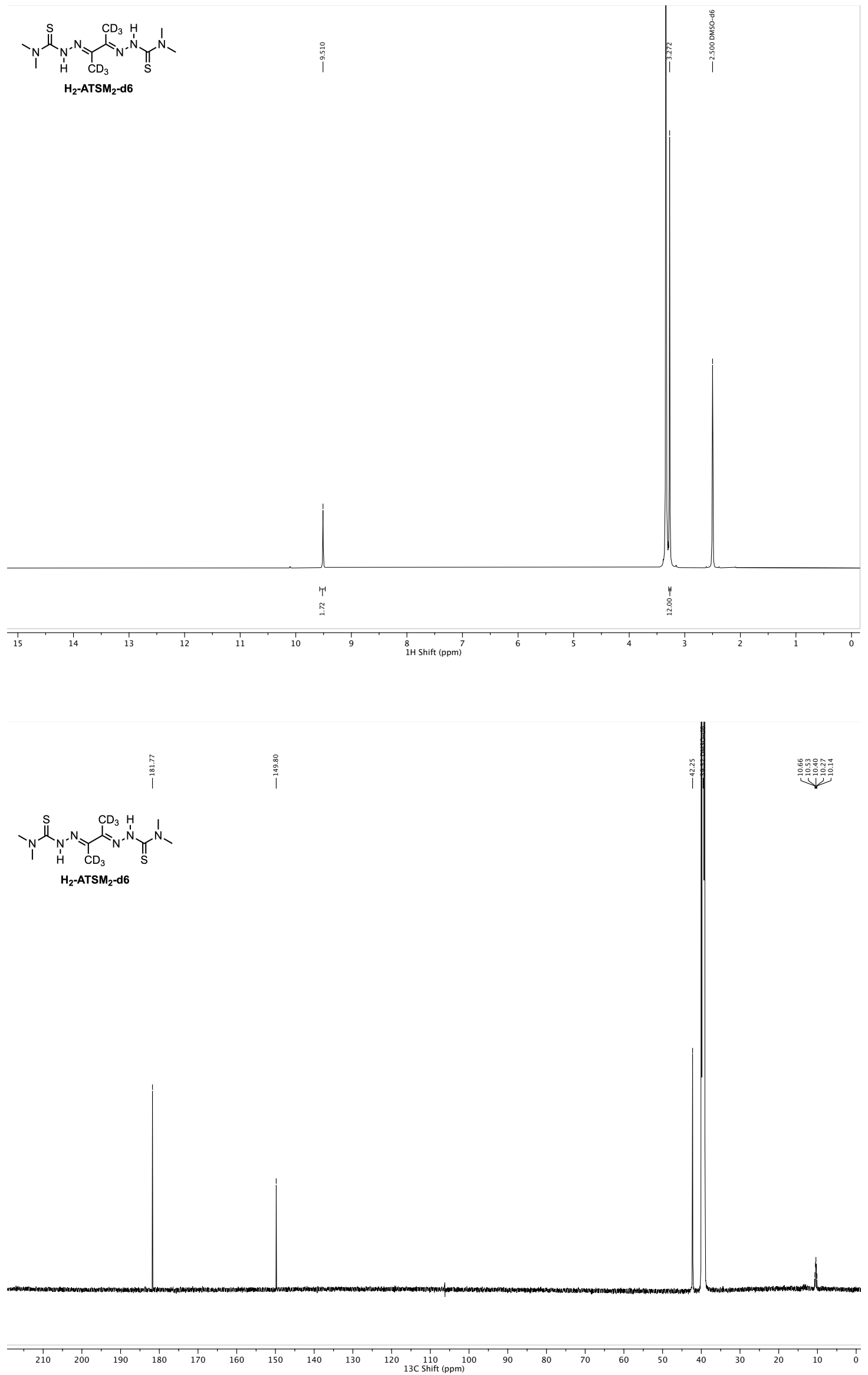

Figure S23. ${ }^{1} \mathrm{H}$ and ${ }^{13} \mathrm{C}$ NMR spectra of $\mathrm{H}_{2}-\mathrm{ATSM}_{2}-\mathrm{d} 6$ in DMSO- $d_{6}$. 


\section{Computational section}

Given the excessive computational resources and time required for the calculation of CuATSM and the other $\mathrm{Cu}$ complexes at the high accuracy CBS-QB3 level of theory, we turned to DFT methods. Different types of exchange correlation functionals (i.e. local or hybrid, GGA or metaGGA, with or without D3 dispersion correction) in combination with different basis sets including def2TZVP, CBSB7 and CBSB7+ were benchmarked with the purpose of identifying a methodology which yielded comparable predictions to CBS-QB3. Complex S1 (Figure S24) was chosen as a surrogate for CuATSM in these benchmarking calculations.

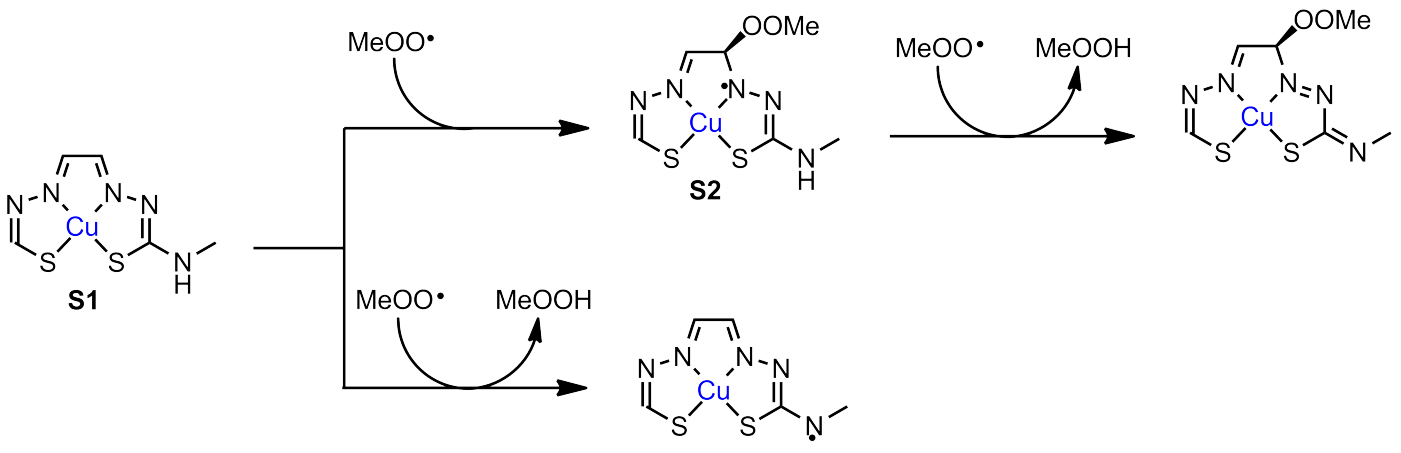

Figure S24. HAT and addition peroxyl radicals to truncated analogue of CuATSM.

The computed N-H BDE and the corresponding $\Delta$ G for HAT from $\mathbf{S 1}$ as well as peroxyl radical addition to $\mathbf{S 1}$ and subsequent HAT from S2 are summarized in Table 1 and 2. Additionally, the corresponding O-H BDEs of $\mathrm{MeOOH}$ calculated using different methods were also included for reference purposes. Of the methods which were surveyed, MN15/CBSB7+ was found to be most comparable to CBS-QB3 in profiling the BDE and thermodynamics of S1, with differences of only $-0.7,1.0$ and $+2.0 \mathrm{kcal} / \mathrm{mol}$ in N-H BDE, $\Delta \mathrm{G}$ for HAT and $\Delta \mathrm{G}$ for peroxyl radical addition, respectively. The basis sets CBSB7 and def2tzvp gave virtually identical energies whereas introduction of a diffuse functional (i.e. CBSB7+) was found to be essential in providing the best agreement. Notably, although N-H BDEs computed using wB97X was close to that obtained at CBS-QB3 level of theory, the O-H BDE of MeOOH was severely underestimated. This was also true of B3LYP and M06-L.

Geometry optimizations and energy calculations of the reactants, products and TS structures were performed as implemented in the Gaussian 16 suite of programs with Opt = Tight and Int = Ultrafine used for convergence criteria (i.e. threshold $=0.000015$ and 0.000060 for maximum force and displacement, respectively). Local maxima for TS structures and that the TS structure connects to the corresponding reactants and products were confirmed by frequency calculations and IRC calculations, respectively. 
Table S1. Benchmark of various methods

\begin{tabular}{|c|c|c|c|c|c|c|}
\hline Methods & $\begin{array}{c}\text { O-H BDE of } \\
\mathrm{MeOOH}(\mathrm{kcal} / \mathrm{mol})\end{array}$ & $\begin{array}{c}\Delta \mathrm{BDE} \text { compared } \\
\text { to } \mathrm{CBS}-\mathrm{QB} 3 \\
\mathrm{BDE}\end{array}$ & $\begin{array}{l}\text { N-H BDE of } \mathbf{S 1} \\
(\mathrm{kcal} / \mathrm{mol})\end{array}$ & $\begin{array}{c}\Delta \mathrm{BDE} \text { compared } \\
\text { to CBS-QB3 } \\
\text { BDE }\end{array}$ & $\begin{array}{c}\text { N-H BDE of } \mathbf{S 2} \\
(\mathrm{kcal} / \mathrm{mol})\end{array}$ & $\begin{array}{c}\Delta \mathrm{BDE} \text { compared } \\
\text { to } \mathrm{CBS}-\mathrm{QB} 3 \\
\mathrm{BDE}\end{array}$ \\
\hline CBS-QB3 & 86.2 & 0.0 & 99.2 & 0.0 & 77.8 & 0.0 \\
\hline B3LYP/CBSB7 & 79.6 & -6.6 & 95.8 & -3.4 & 81.0 & +3.2 \\
\hline wB97X/CBSB7 & 79.0 & -7.2 & 100.0 & +0.7 & 83.0 & +5.2 \\
\hline M06-2X/def2TZVP & 83.0 & -3.2 & 101.5 & +2.2 & 80.7 & +2.9 \\
\hline M06-2X/CBSB7+ & 83.7 & -2.5 & 101.1 & +1.9 & 81.4 & +3.6 \\
\hline M06-2X-D3/CBSB7 & 83.0 & -3.2 & 102.5 & +3.3 & 82.2 & +4.4 \\
\hline M06-2X/CBSB7 & 83.0 & -3.2 & 103.0 & +3.8 & 82.1 & +4.3 \\
\hline M06/CBSB7 & 82.4 & -3.8 & 98.0 & -1.2 & 80.3 & +2.5 \\
\hline M06/CBSB7+ & 83.3 & -2.9 & 96.7 & -2.5 & 79.8 & +2.0 \\
\hline M06/ def2TZVP & 81.9 & -4.3 & 96.7 & -2.6 & 78.9 & +1.1 \\
\hline M06-L/CBSB7 & 76.8 & -9.4 & 91.0 & -8.2 & 75.1 & -2.7 \\
\hline M06-L/CBSB7+ & 77.9 & -8.3 & 89.9 & -9.3 & 74.9 & -2.9 \\
\hline M06-L/ def2TZVP & 76.9 & -9.3 & 90.4 & -8.8 & 74.7 & -3.1 \\
\hline MN15/CBSB7 & 83.1 & -3.1 & 99.7 & +0.5 & 80.9 & +3.1 \\
\hline MN15/CBSB7+ & 84.0 & -2.2 & 98.5 & -0.7 & 80.0 & +2.2 \\
\hline MN15/ def2TZVP & 83.0 & -3.1 & 98.3 & -0.9 & 79.5 & +1.7 \\
\hline MN15-L/CBSB7 & 82.2 & -4.0 & 93.0 & -6.2 & 74.8 & -3.0 \\
\hline MN15-L/CBSB7+ & 83.2 & -3.0 & 92.6 & -6.6 & 74.7 & -3.0 \\
\hline MN15-L/def2TZVP & 82.6 & -3.6 & 92.2 & -7.0 & 74.0 & -3.8 \\
\hline
\end{tabular}


Table S2. Benchmark of various methods

\begin{tabular}{|c|c|c|c|c|c|c|}
\hline Methods & $\begin{array}{l}\Delta \mathrm{G} \text { for HAT of } \\
\text { S1 }(\mathrm{kcal} / \mathrm{mol})\end{array}$ & $\begin{array}{c}\Delta \Delta \mathrm{G} \text { compared to } \\
\text { CBS-QB3 } \Delta \mathrm{G} \\
(\mathrm{kcal} / \mathrm{mol})\end{array}$ & $\begin{array}{l}\Delta \mathrm{G} \text { for addition } \\
\text { of peroxyl to } \mathbf{S 1} \\
(\mathrm{kcal} / \mathrm{mol})\end{array}$ & $\begin{array}{c}\Delta \Delta \mathrm{G} \text { compared to } \\
\text { CBS-QB3 } \Delta \mathrm{G} \\
(\mathrm{kcal} / \mathrm{mol})\end{array}$ & $\begin{array}{c}\Delta \mathrm{G} \text { for } \\
\text { subsequent HAT } \\
\text { of } \mathbf{S 2}(\mathrm{kcal} / \mathrm{mol})\end{array}$ & $\begin{array}{c}\Delta \Delta \mathrm{G} \text { compared to } \\
\text { CBS-QB3 } \Delta \mathrm{G} 3 \\
(\mathrm{kcal} / \mathrm{mol})\end{array}$ \\
\hline CBS-QB3 & 13.5 & 0.0 & -9.0 & 0.0 & -7.8 & 0.0 \\
\hline B3LYP/CBSB7 & 16.7 & +3.2 & 1.5 & +10.4 & 0.5 & +8.3 \\
\hline wB97X/CBSB7 & 21.2 & +7.7 & -6.7 & +2.2 & 4.7 & +12.5 \\
\hline M06-2X/def2TZVP & 18.5 & +5.0 & -6.1 & +2.3 & -2.7 & +5.1 \\
\hline M06-2X/CBSB7+ & 16.8 & +3.4 & -7.5 & +1.4 & -2.6 & +5.2 \\
\hline M06-2X-D3/CBSB7 & 19.6 & +6.1 & -8.5 & +0.4 & -0.5 & +7.3 \\
\hline M06-2X/CBSB7 & 20.1 & +6.6 & -8.1 & +0.8 & -0.5 & +7.3 \\
\hline M06/CBSB7 & 16.4 & +3.0 & -6.2 & +2.8 & -1.3 & +6.5 \\
\hline M06/CBSB7+ & 13.8 & +0.3 & -5.3 & +3.6 & -3.1 & +4.7 \\
\hline M06/def2TZVP & 15.1 & +1.6 & -3.9 & +5.1 & -2.4 & +5.4 \\
\hline M06-L/CBSB7 & 15.2 & +1.7 & 0.2 & +9.1 & -1.1 & +6.7 \\
\hline M06-L/CBSB7+ & 12.7 & -0.9 & -1.8 & +7.1 & -2.5 & +5.3 \\
\hline M06-L/def2TZVP & 14.3 & +0.8 & 1.5 & +10.5 & -1.3 & +6.5 \\
\hline MN15/CBSB7 & 16.8 & +3.3 & -7.7 & +1.5 & -1.7 & +6.1 \\
\hline MN15/CBSB7+ & 14.5 & +1.0 & -7.0 & +2.0 & -3.5 & +4.4 \\
\hline MN15/ def2TZVP & 15.5 & +2.0 & -6.4 & +2.5 & -3.0 & +4.8 \\
\hline MN15-L/CBSB7 & 11.5 & -2.0 & -5.4 & +3.5 & -6.1 & +1.7 \\
\hline MN15-L/CBSB7+ & 8.0 & -5.5 & -5.8 & +3.2 & -7.5 & +0.3 \\
\hline MN15-L/def2TZVP & 8.5 & -5.0 & -5.4 & +3.6 & -7.6 & +0.2 \\
\hline
\end{tabular}


<smiles>CNC1=NN2CCN3N=CS[C@@]32S1</smiles>

$\mathrm{N}-\mathrm{H}$ BDE $=99.2$

$\mathrm{N}-\mathrm{H}$ BDE $=98.5$

$\mathrm{N}-\mathrm{H}$ BDE $=95.8$

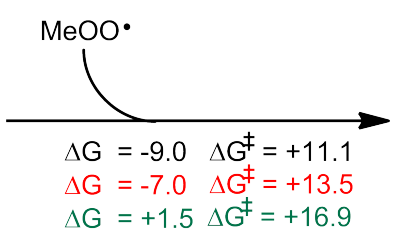

$\mathrm{MeOO} \cdot$<smiles>NC1=NN2C=CN3N=CS[C@@]32S1</smiles>

$\mathrm{N}-\mathrm{H}$ BDE $=98.0$

$\mathrm{N}-\mathrm{H}$ BDE $=98.1$

$\mathrm{N}-\mathrm{H}$ BDE $=96.0$

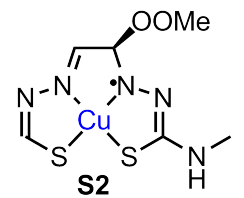

N-H BDE $=77.8$

$\mathrm{N}-\mathrm{H}$ BDE $=80.0$ $\mathrm{N}-\mathrm{H}$ BDE $=81.0$
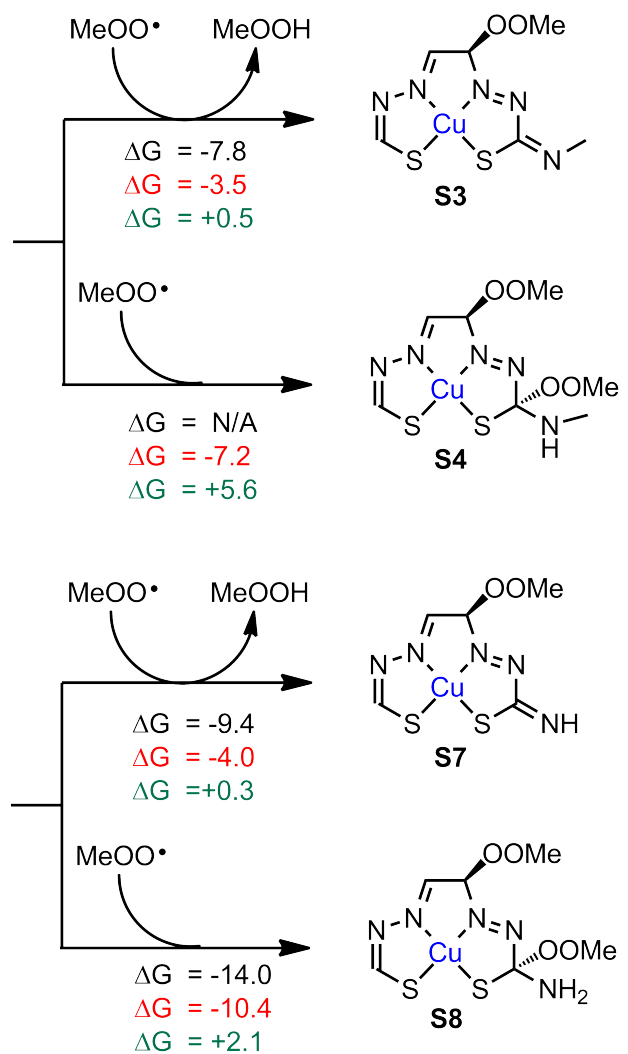<smiles>NC1=NN[Si]2(NNN=CS2)S1</smiles>

N-H BDE $=76.6$

$\mathrm{N}-\mathrm{H}$ BDE $=79.8$ $\mathrm{N}-\mathrm{H}$ BDE $=79.7$

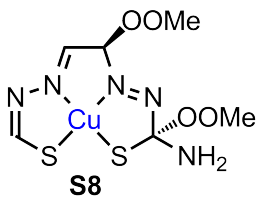<smiles>CC1=CN2N=CS[C@]23SC(N)=NN13</smiles>
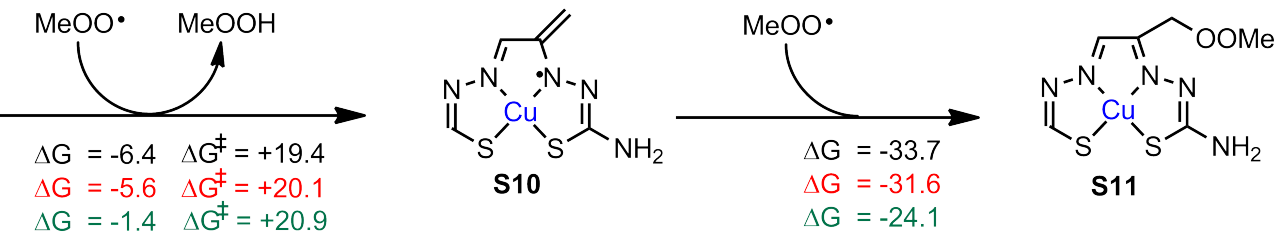

C- $\mathrm{H}$ BDE $=78.9$

C-H BDE $=77.5$

C-H BDE $=77.3$

Figure S25. Free energy profile of radical-trapping for truncated analogues derived from CuATSM computed using CBS-QB3 (O), MN15/CBSB7+ (○) or B3LYP/CBSB7(○).<smiles>CNC1=NC2(SC(C)C)C(NC)=NN3N=NC(C)=C(C)C32S1</smiles>

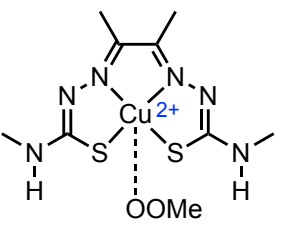<smiles>C1CCCCC1</smiles>

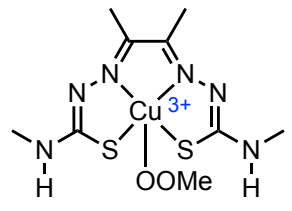<smiles>C1CCCCC1</smiles>

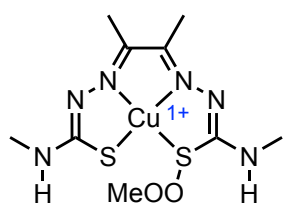



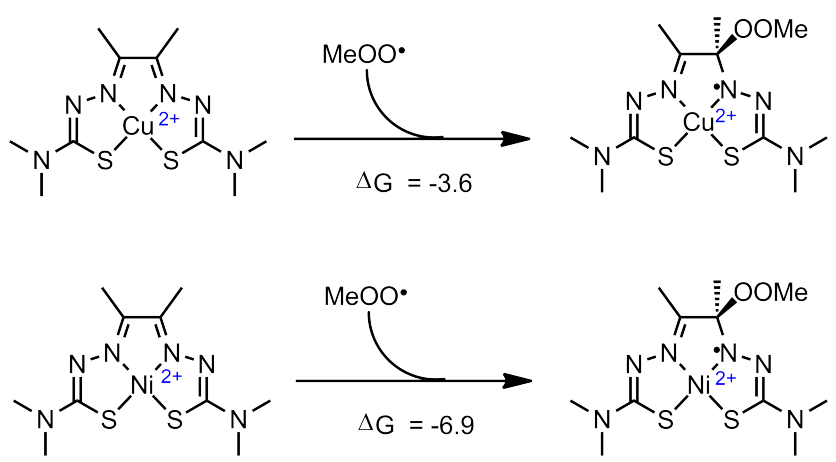

Figure S26. MN15/CBSB7+ computed free energies (in $\mathrm{kcal} / \mathrm{mol}$ ) predict that radical addition to $\mathrm{NiATSM}_{2}$ in THF is less reversible than to $\mathrm{CuATSM}_{2}$.

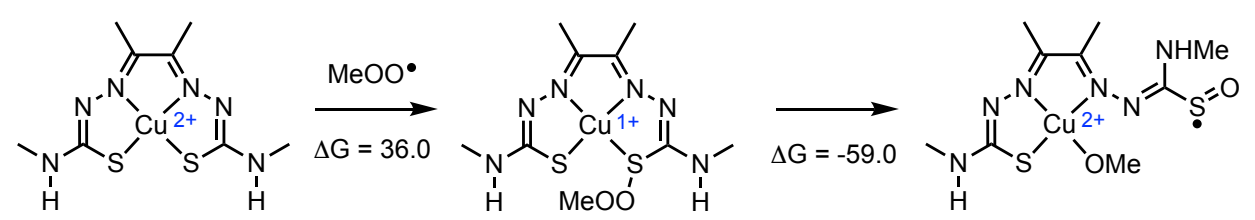

Figure S27. MN15/CBSB7+ computed free energies (in $\mathrm{kcal} / \mathrm{mol}$ ) for substitution at the S-atom of CuATSM in THF at $298 \mathrm{~K}$ and subsequent inner-sphere electron transfer to yield a sulfinyl radical. 

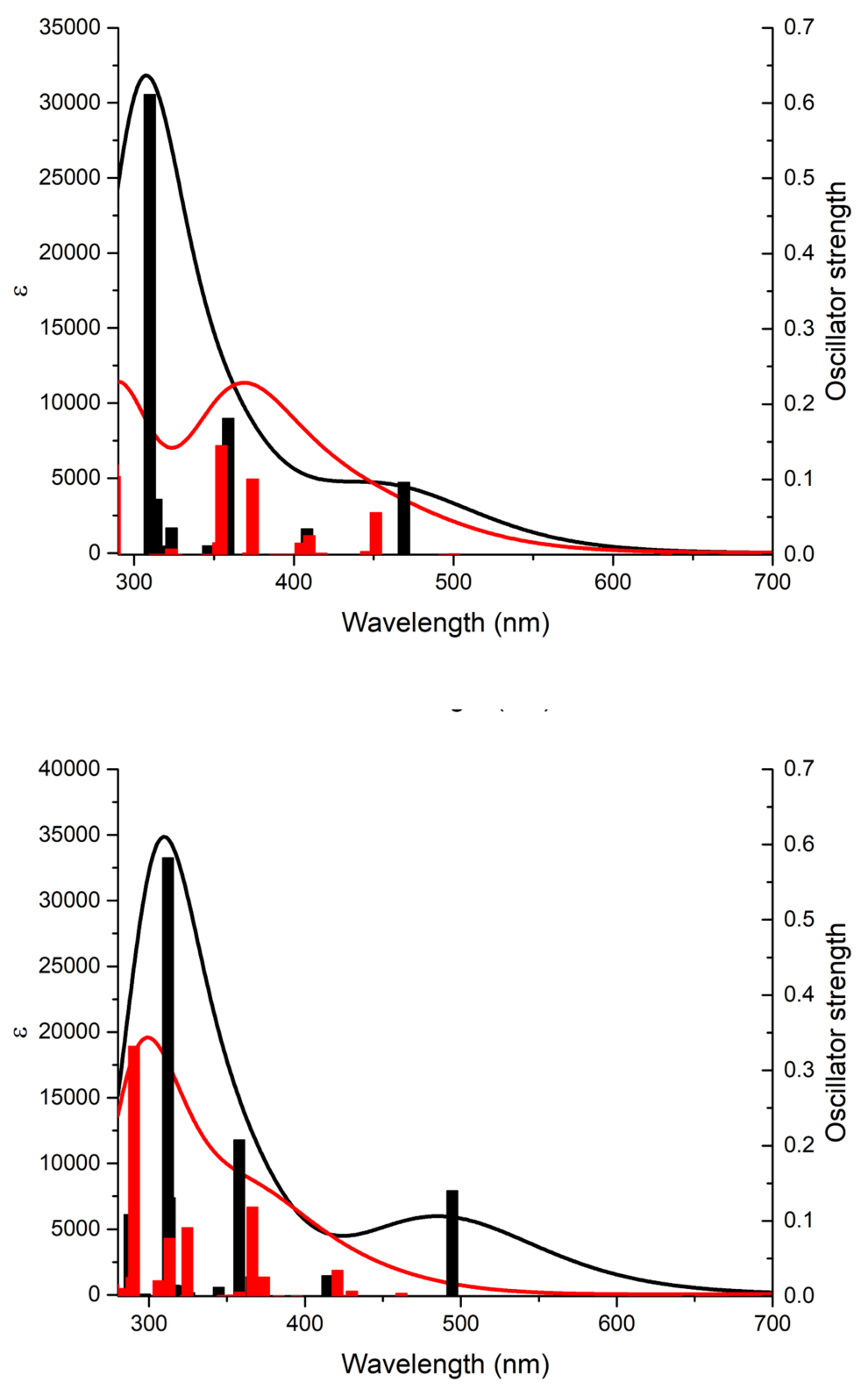

Figure S28. Predicted UV-Vis spectra of CuATSM (top, black) and ATSM 2 (bottom, black) and the presumed intermediates formed therefrom (red) during the inhibited autoxidations. TD-DFT calculations of the first 30 excited states were carried out at the MN15/CBSB7+ level of theory in THF (CPCM) at $298 \mathrm{~K}$. 


\section{Cartesian coordinates of relevant stationary points}

\section{CuATSM}<smiles>CNC1=NN2C(C)=C(C)N3N=C(NC)SC23S1</smiles>

\section{CBS-QB3 Enthalpy= $\quad-3075.639774$ CBS-QB3 Free Energy= $\quad-3075.710092$}

$\begin{array}{lrrr}\mathrm{O}^{2} & & & \\ \mathrm{Cu} & 0.00665800 & -0.69228800 & -0.00008300 \\ \mathrm{~S} & 1.85897200 & -2.00132600 & -0.00028500 \\ \mathrm{C} & 2.96246700 & -0.62225700 & -0.00014300 \\ \mathrm{~N} & 2.62341800 & 0.65965300 & 0.00002400 \\ \mathrm{~N} & 1.28563200 & 0.83000000 & 0.00007400 \\ \mathrm{C} & 0.73377500 & 2.00966500 & 0.00023400 \\ \mathrm{C} & -0.74371400 & 1.99780600 & 0.00026700 \\ \mathrm{~N} & -1.27890300 & 0.81131600 & 0.00013000 \\ \mathrm{~N} & -2.61572800 & 0.64639100 & 0.00013400 \\ \mathrm{C} & -2.95460200 & -0.63816300 & -0.00001500 \\ \mathrm{~S} & -1.84726200 & -2.01211000 & -0.00020300 \\ \mathrm{~N} & -4.27172700 & -0.92248500 & -0.00003200 \\ \mathrm{C} & -5.32013400 & 0.08543500 & 0.00012400 \\ \mathrm{H} & -5.25045400 & 0.72421100 & -0.88401600 \\ \mathrm{H} & -6.28474300 & -0.42221700 & 0.00002000 \\ \mathrm{H} & -5.25049000 & 0.72391500 & 0.88448200 \\ \mathrm{H} & -4.52146500 & -1.89712800 & -0.00012900 \\ \mathrm{C} & -1.58085000 & 3.23975500 & 0.00043600 \\ \mathrm{H} & -0.97633100 & 4.14501700 & 0.00066300 \\ \mathrm{H} & -2.23740400 & 3.24696500 & -0.87440100 \\ \mathrm{H} & -2.23755600 & 3.24661500 & 0.87515800 \\ \mathrm{C} & 1.52209700 & 3.28387900 & 0.00037400 \\ \mathrm{H} & 1.28992200 & 3.88890400 & 0.88242300 \\ \mathrm{H} & 2.58418500 & 3.05067900 & 0.00029200 \\ \mathrm{H} & 1.28983700 & 3.88915000 & -0.88148300 \\ \mathrm{~N} & 4.28014400 & -0.90868900 & -0.00022100 \\ \mathrm{C} & 5.33138000 & 0.09584900 & -0.00010600 \\ \mathrm{H} & 5.26496900 & 0.73428800 & 0.88461700 \\ \mathrm{H} & 6.29437800 & -0.41495500 & -0.00025300 \\ \mathrm{H} & 5.26486000 & 0.73459100 & -0.88460000 \\ \mathrm{H} & 4.52748400 & -1.88382000 & -0.00032200 \\ & & & \end{array}$

Aminyl radical derived from CuATSM<smiles>CNC1=NN2C(C)=C(C)N3N=C(NC)SC23S1</smiles>

\section{CBS-QB3 Enthalpy= $\quad-3074.987228$ CBS-QB3 Free Energy $=\quad-3075.059656$}

03

$\mathrm{Cu}$ 
$-2.02507400$

$-0.68404600$

0.66293000

0.79368700

2.02267500

2.00197400

0.78529900

0.65235600

$-0.64783500$

$-2.02065700$

$-0.86402800$

0.18357700

0.81650000

$-0.29299200$

0.81651100

$-1.82797500$

3.23223100

4.14316000

3.23263400

3.23263700

3.28113300

3.89041100

3.03384000

3.89040100

$-0.96835100$

0.05327100

1.08288000

$-0.10487000$

$-0.10486900$
-0.00000100
0.00000100
0.00000000
-0.00000100
-0.00000200
-0.00000100
-0.00000100
0.00000000
0.00000000
-0.00000200
0.00000000
0.00000700
0.88514700
0.00000400
-0.88512500
0.00000400
0.00000000
0.00000200
0.87521400
-0.87521600
-0.00000200
-0.88225700
-0.00001400
0.88226400
0.00000300
0.00000600
0.00000400
-0.87577300
0.87578900

\section{$\mathrm{H}_{2}$-ATSM}<smiles>CNC(=S)N/N=C(C)/C(C)=N/NC(=S)NC</smiles>

\section{CBS-QB3 Enthalpy= CBS-QB3 Free Energy=}

$-1437.303570$ $-1437.376164$

\begin{abstract}
4.28186400
4.09874400

1.71668500

0.62240100

$-0.62240100$

$-1.71668500$

$-4.09874400$

$-4.28186500$

$-5.17508800$

$-5.17202500$

$-4.68574800$

$-6.20531900$

$-4.68577200$

$-6.05571700$

$-0.54744600$

0.48928200

$-1.03977000$

$-1.03977600$

0.54744600

1.03977700

1.03977000

$-0.48928100$
\end{abstract}

1.93813200

0.27977800

0.28685200

$-0.39671500$

0.39671300

$-0.28685400$

$-0.27977800$

$-1.93813200$

0.55285100

2.00561800

2.41779700

2.34968200

2.41773100

0.06457500

1.90260800

2. 22932400

2. 31727400

2. 31728600

$-1.90260900$

$-2.31728700$

$-2.31727500$

$-2.22932600$
$-0.00001300$

0.00000300

0.00000000

0.00000200

$-0.00000600$

$-0.00000300$

$-0.00001000$

$-0.00001200$

$-0.00001300$

0.00004300

$-0.89246800$

0.00003900

0.89259800

0.00000500

$-0.00001600$

$-0.00001500$

$-0.88754600$

0.88750700

0.00001200

$-0.88751000$

0.88754200

0.00001000 


$\begin{array}{lrrr}\mathrm{N} & 5.17508900 & -0.55284900 & 0.00001500 \\ \mathrm{C} & 5.17202700 & -2.00561600 & 0.00000800 \\ \mathrm{H} & 4.68577500 & -2.41776000 & -0.89253300 \\ \mathrm{H} & 6.20532200 & -2.34968000 & 0.00002300 \\ \mathrm{H} & 4.68575000 & -2.41776600 & 0.89253300 \\ \mathrm{H} & 6.05571800 & -0.06457300 & 0.00000200 \\ \mathrm{~N} & 2.88892400 & -0.38253800 & 0.00000700 \\ \mathrm{H} & 2.87960200 & -1.39534100 & 0.00001200 \\ \mathrm{~N} & -2.88892400 & 0.38253700 & -0.00000800 \\ \mathrm{H} & -2.87960100 & 1.39534000 & -0.00001500\end{array}$

Aminyl radical derived from $\mathrm{H}_{2}$-ATSM<smiles>CNC(=S)N/N=C(C)/C(C)=N/NC(=S)NC</smiles>

\section{CBS-QB3 Enthalpy= CBS-QB3 Free Energy=}

$-1436.651592$

$-1436.727068$

$$
\begin{array}{r}
4.24749000 \\
4.03819400 \\
1.65726100 \\
0.54778500 \\
-0.67529200 \\
-1.79931900 \\
-4.14418600 \\
-3.93591800 \\
-5.32508700 \\
-5.57161300 \\
-5.15437600 \\
-6.64676500 \\
-5.15436400 \\
-0.57104000 \\
0.47136100 \\
-1.05829900 \\
-1.05829200 \\
0.44736800 \\
0.93200300 \\
0.93200200 \\
-0.59201500 \\
5.09942000 \\
5.07164900 \\
4.57883300 \\
6.09892800 \\
4.57882900 \\
5.98858700 \\
2.81584500 \\
2.79091800 \\
-2.95852400 \\
-2.97687000
\end{array}
$$

0.00000400

0.00000000

$-0.00000200$

$-0.00000500$

$-0.00000200$

$-0.00000500$

$-0.00000800$

0.00001500

$-0.00001500$

$-0.00000900$

0.89088100

$-0.00001600$

$-0.89088800$

0.00000500

0.00000900

0.88689800

$-0.88688900$

$-0.00000900$

0.88737100

$-0.88739400$

$-0.00001000$

0.00000000

0.00001100

0.89276200

0.00001000

$-0.89273200$

0.00000700

$-0.00000200$

$-0.00000700$

$-0.00000200$

$-0.00001200$

\section{$\mathrm{H}_{2}$-ATSM tautomer}<smiles></smiles>

\section{CBS-QB3 Enthalpy= CBS-QB3 Free Energy=}


01

$\begin{array}{lrrr}\mathrm{C} & -3.98067100 & -0.14006400 & -0.00805100 \\ \mathrm{~N} & -1.73416100 & -0.21175100 & -0.00404600 \\ \mathrm{C} & -0.60476100 & 0.42220800 & -0.00287400 \\ \mathrm{C} & 0.60476300 & -0.42221200 & -0.00287500 \\ \mathrm{~N} & 1.73416200 & 0.21174800 & -0.00404800 \\ \mathrm{C} & 3.98067100 & 0.14006400 & -0.00805400 \\ \mathrm{~N} & 5.17315700 & -0.50475400 & -0.04289100 \\ \mathrm{C} & 5.27861600 & -1.95430800 & 0.02824700 \\ \mathrm{H} & 4.90798600 & -2.34443600 & 0.98170500 \\ \mathrm{H} & 4.69743900 & -2.41282900 & -0.77296700 \\ \mathrm{H} & 6.32738000 & -2.22788800 & -0.09050800 \\ \mathrm{H} & 5.99978300 & 0.04381800 & 0.12396600 \\ \mathrm{C} & 0.46423200 & -1.92092500 & -0.00213700 \\ \mathrm{H} & -0.09018700 & -2.24897300 & 0.88076900 \\ \mathrm{H} & -0.10465700 & -2.24880700 & -0.87557000 \\ \mathrm{H} & 1.44683100 & -2.38472200 & -0.00894600 \\ \mathrm{C} & -0.46422900 & 1.92092100 & -0.00213800 \\ \mathrm{H} & 0.10466100 & 2.24880100 & -0.87557000 \\ \mathrm{H} & -1.44682800 & 2.38471800 & -0.00894800 \\ \mathrm{H} & 0.09018900 & 2.24897000 & 0.88076900 \\ \mathrm{~N} & -5.17315500 & 0.50475900 & -0.04289100 \\ \mathrm{C} & -5.27860900 & 1.95431300 & 0.02824600 \\ \mathrm{H} & -6.32737300 & 2.22789600 & -0.09050300 \\ \mathrm{H} & -4.90797200 & 2.34444000 & 0.98170100 \\ \mathrm{H} & -4.69743600 & 2.41283300 & -0.77297200 \\ \mathrm{~N} & -2.87682700 & 0.54363500 & 0.00052600 \\ \mathrm{~N} & 2.87682900 & -0.54363700 & 0.00052300 \\ \mathrm{~S} & -4.09640200 & -1.92651000 & -0.00305900 \\ \mathrm{H} & -2.74476900 & -2.00650700 & 0.04430100 \\ \mathrm{~S} & 4.09639500 & 1.92651100 & -0.00305800 \\ \mathrm{H} & 2.74476100 & 2.00650400 & 0.04430200 \\ \mathrm{H} & -5.99978400 & -0.04381100 & 0.12395900 \\ & & & \end{array}$

\section{Aminyl radical derived from $\mathrm{H}_{2}$-ATSM tautomer}<smiles></smiles>

\section{CBS-QB3 Enthalpy= CBS-QB3 Free Energy=}

$-1436.661622$ $-1436.735772$

\begin{abstract}
4.07717800
1.79693700

0.62954000

$-0.56292000$

$-1.71258100$

$-3.95448200$

$-5.09885700$

$-5.13352300$

$-4.63417100$

$-6.17651700$

$-4.63416900$

$-5.96875500$

$-0.50195500$

0.52501900

$-1.02522000$

$-1.02518800$

0.58183500

1. 10991800

1.10995000
\end{abstract}

0.12548900

0.23179800

$-0.43263100$

0.37299200

$-0.27441500$

$-0.13809300$

0.57139400

2.02872100

2.42960400

2.34391900

2.42961900

0.06554100

1.87650200

2.22561300

2. 26827700

2.26829700

$-1.93240800$

$-2.32151400$

$-2.32150300$
0.00000000

$-0.00000200$

0.00000200

$-0.00000500$

0.00000400

0.00000100

$-0.00000100$

$-0.00001500$

$-0.88454000$

$-0.00001600$

0.88450300

$-0.00000900$

$-0.00002300$

$-0.00004400$

$-0.87585900$

0.87582500

0.00001500

$-0.87443400$

0.87445000 
$-2.29271400$

$-0.50760500$

$-1.95613200$

$-2.44732500$

$-2.27739800$

$-2.27742300$

$-0.52863200$

0.50679000

1. 91775500

2.13783300

$-1.90793800$

$-2.04983700$
0.00003600

$-0.00000900$

$-0.00002900$

$-0.00003600$

$-0.87583300$

0.87576700

$-0.00000800$

$-0.00000400$

0.00002500

0.00002700

0.00001300

0.00001200

\section{CuATSM (THF)}<smiles>CNC1=NN2C(C)=C(C)N3N=C(NC)SC23S1</smiles>

\section{MN15/CBSB7+ Enthalpy= MN15/CBSB7+ Free Energy=}

\section{$-3078.065848$ $-3078.137269$}

${ }^{O} 2$
$\mathrm{Cu}$
$\mathrm{S}$
$\mathrm{C}$
$\mathrm{N}$
$\mathrm{N}$
$\mathrm{C}$
$\mathrm{C}$
$\mathrm{N}$
$\mathrm{N}$
$\mathrm{C}$
$\mathrm{S}$
$\mathrm{N}$
$\mathrm{C}$
$\mathrm{H}$
$\mathrm{H}$
$\mathrm{H}$
$\mathrm{H}$
$\mathrm{C}$
$\mathrm{H}$
$\mathrm{H}$
$\mathrm{H}$
$\mathrm{C}$
$\mathrm{H}$
$\mathrm{H}$
$\mathrm{H}$
$\mathrm{N}$
$\mathrm{C}$
$\mathrm{H}$
$\mathrm{H}$
$\mathrm{H}$
$\mathrm{H}$
$\mathrm{H}$

$$
\begin{array}{r}
0.00822500 \\
1.85336900 \\
2.96342600 \\
2.62806100 \\
1.28500800 \\
0.73702500 \\
-0.74761900 \\
-1.27681900 \\
-2.61806300 \\
-2.95334800 \\
-1.83758900 \\
-4.26329700 \\
-5.30216700 \\
-5.22385700 \\
-6.27021900 \\
-5.22380300 \\
-4.51876700 \\
-1.57855700 \\
-0.97059600 \\
-2.23136900 \\
-2.23147500 \\
1.50345400 \\
1.25001600 \\
2.57106100 \\
1.24992900 \\
4.27472800 \\
5.31384000 \\
5.23666500 \\
6.28173100 \\
5.23650100 \\
4.52951500
\end{array}
$$

$$
\begin{array}{r}
-0.00007700 \\
-0.00027600 \\
-0.00015200 \\
0.00002300 \\
0.00007700 \\
0.00023600 \\
0.00027000 \\
0.00013500 \\
0.00013400 \\
-0.00001400 \\
-0.00019600 \\
-0.00003100 \\
0.00009800 \\
-0.88446200 \\
0.00007800 \\
0.88478200 \\
-0.00016700 \\
0.00043300 \\
0.00062100 \\
-0.87639600 \\
0.87718300 \\
0.00037800 \\
0.88137700 \\
0.00029800 \\
-0.88042700 \\
-0.00023600 \\
-0.00012100 \\
0.88467900 \\
-0.00030800 \\
-0.88465600 \\
-0.00031800
\end{array}
$$

\section{TS structure for HAT of CuATSM (THF)}




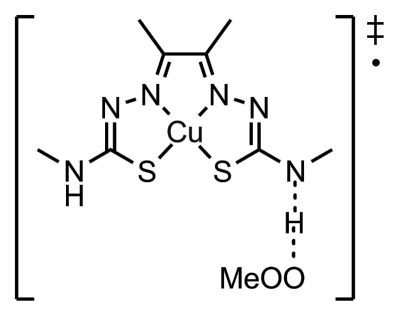

\section{MN15/CBSB7+ Enthalpy $=-3268.070358$ \\ MN15/CBSB7+ Free Energy= $\quad-3268.147777$ \\ Imaginary frequency: -1079.59}

$\begin{array}{lrrr}03 & & \\ \mathrm{Cu} & 0.72026700 & -0.49459700 & -0.06645200 \\ \mathrm{~S} & 2.14158600 & -2.28062500 & -0.09429300 \\ \mathrm{C} & 3.58125200 & -1.27974500 & 0.02178000 \\ \mathrm{~N} & 3.59074500 & 0.06688100 & 0.08342200 \\ \mathrm{~N} & 2.37116600 & 0.58328600 & 0.04585700 \\ \mathrm{C} & 2.18947500 & 1.89278500 & 0.09203000 \\ \mathrm{C} & 0.80647700 & 2.31488000 & 0.04902100 \\ \mathrm{~N} & -0.07505500 & 1.32055900 & -0.03126400 \\ \mathrm{~N} & -1.36808300 & 1.54974100 & -0.05564100 \\ \mathrm{C} & -2.11600100 & 0.41028700 & -0.16500200 \\ \mathrm{~S} & -1.41100500 & -1.22382700 & -0.15056000 \\ \mathrm{~N} & -3.41531700 & 0.52839600 & -0.30083400 \\ \mathrm{C} & -4.08512400 & 1.82578800 & -0.26261800 \\ \mathrm{H} & -3.76534600 & 2.42804000 & 0.59067100 \\ \mathrm{H} & -3.88119800 & 2.39250200 & -1.17570500 \\ \mathrm{H} & -5.15626000 & 1.63849000 & -0.20181600 \\ \mathrm{C} & 0.40187800 & 3.75290800 & 0.09571500 \\ \mathrm{H} & 0.87284800 & 4.31290900 & -0.71679200 \\ \mathrm{H} & -0.67773800 & 3.83984200 & 0.00912600 \\ \mathrm{H} & 0.72007400 & 4.21257200 & 1.03625600 \\ \mathrm{C} & 3.34306500 & 2.83877700 & 0.18354900 \\ \mathrm{H} & 3.01884900 & 3.87439100 & 0.25073300 \\ \mathrm{H} & 3.95331100 & 2.59793800 & 1.05812600 \\ \mathrm{H} & 3.98985300 & 2.72527400 & -0.69126300 \\ \mathrm{~N} & 4.77093300 & -1.88508000 & 0.05358100 \\ \mathrm{C} & 6.05395900 & -1.19389600 & 0.14720100 \\ \mathrm{H} & 6.10678800 & -0.59738700 & 1.05968700 \\ \mathrm{H} & 6.83993600 & -1.94628200 & 0.16308900 \\ \mathrm{H} & 6.20302600 & -0.53417700 & -0.70961300 \\ \mathrm{H} & 4.77115300 & -2.89250700 & 0.00689300 \\ \mathrm{H} & -4.12717200 & -0.44465700 & -0.15049900 \\ \mathrm{O} & -4.90764400 & -1.37094000 & 0.17421800 \\ \mathrm{O} & -6.15926300 & -0.89340900 & -0.28751100 \\ \mathrm{C} & -7.14477000 & -1.25972000 & 0.66658000 \\ \mathrm{H} & -6.93959400 & -0.79066700 & 1.63425300 \\ \mathrm{H} & -8.09450500 & -0.89854600 & 0.26676500 \\ \mathrm{H} & -7.17779600 & -2.34726300 & 0.78581900\end{array}$

\section{Aminyl radical derived from CuATSM (THF)}<smiles>CNC1=NN2C(C)=C(C)N3N=C(NC)SC23S1</smiles>

MN15/CBSB7+ Enthalpy $=\quad-3077.418395$

$\mathrm{MN15/CBSB7}+$ Free Energy $=\quad-3077.489534$ 
03

$\begin{array}{lrrr}\mathrm{Cu} & 0.03689000 & -0.73577200 & 0.00000100 \\ \mathrm{~S} & 1.84999800 & -2.00792700 & 0.00000300 \\ \mathrm{C} & 3.03095600 & -0.68483500 & -0.00000600 \\ \mathrm{~N} & 2.60051000 & 0.65607700 & -0.00000300 \\ \mathrm{~N} & 1.32694500 & 0.78692100 & -0.00000100 \\ \mathrm{C} & 0.74870500 & 2.01152400 & 0.00000100 \\ \mathrm{C} & -0.67500900 & 1.99371500 & 0.00000100 \\ \mathrm{~N} & -1.22809200 & 0.78278100 & 0.00000200 \\ \mathrm{~N} & -2.53875600 & 0.64985500 & 0.00000300 \\ \mathrm{C} & -2.91756000 & -0.64669300 & 0.00000500 \\ \mathrm{~S} & -1.84196000 & -2.00139500 & 0.00000200 \\ \mathrm{~N} & -4.23220800 & -0.87200200 & -0.00000200 \\ \mathrm{C} & -5.23428700 & 0.18447500 & -0.00000600 \\ \mathrm{H} & -5.13069600 & 0.81295400 & -0.88564000 \\ \mathrm{H} & -6.21789700 & -0.27893800 & -0.00001200 \\ \mathrm{H} & -5.13070500 & 0.81295200 & 0.88563000 \\ \mathrm{H} & -4.53495900 & -1.83754100 & -0.00000600 \\ \mathrm{C} & -1.52276800 & 3.21945500 & 0.00000100 \\ \mathrm{H} & -0.92390100 & 4.12753500 & -0.00000300 \\ \mathrm{H} & -2.17489800 & 3.21722800 & -0.87718100 \\ \mathrm{H} & -2.17489300 & 3.21723200 & 0.87718600 \\ \mathrm{C} & 1.54879600 & 3.26860100 & 0.00000100 \\ \mathrm{H} & 1.31406100 & 3.87135400 & 0.88126000 \\ \mathrm{H} & 2.61176600 & 3.03964200 & 0.00000700 \\ \mathrm{H} & 1.31407000 & 3.87134800 & -0.88126400 \\ \mathrm{~N} & 4.27846900 & -0.96890700 & -0.00000500 \\ \mathrm{C} & 5.29343900 & 0.06403200 & -0.00000400 \\ \mathrm{H} & 4.90210800 & 1.08313400 & -0.00000300 \\ \mathrm{H} & 5.93290600 & -0.07754200 & 0.87571000 \\ \mathrm{H} & 5.93290700 & -0.07754000 & -0.87571600\end{array}$

TS structure for addition of MeOO• to CuATSM (THF)

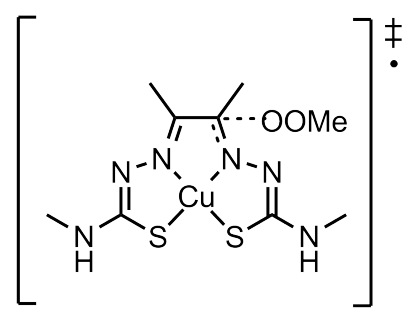

\section{MN15/CBSB7+ Enthalpy $=-3268.086455$ \\ $\mathrm{MN15/CBSB7}+$ Free Energy $=\quad-3268.165926$ Imaginary frequency: -536.84}

O 3
$\mathrm{Cu}$
$\mathrm{S}$
$\mathrm{C}$
$\mathrm{N}$
$\mathrm{N}$
$\mathrm{C}$
$\mathrm{N}$
$\mathrm{N}$
$\mathrm{C}$
$\mathrm{S}$
$\mathrm{N}$
$\mathrm{C}$
$\mathrm{H}$
$\mathrm{H}$

-0.31151400
1.44653900
2.62994500
2.36064900
1.06885000
-0.84749000
-1.46823400
-2.81883400
-3.24036000
-2.22758700
-4.56606700
-5.53218200
-5.35282100
-5.47199600

$$
\begin{array}{r}
-0.92099100 \\
-2.34614900 \\
-1.15079400 \\
0.13506700 \\
0.40839200 \\
1.78050000 \\
0.67172800 \\
0.61313800 \\
-0.60311300 \\
-2.01563500 \\
-0.79188000 \\
0.27979400 \\
1.07573700 \\
0.71203300
\end{array}
$$

$$
\begin{array}{r}
-0.10860100 \\
0.03910500 \\
-0.38952600 \\
-0.66086900 \\
-0.53076300 \\
-0.58482400 \\
-0.35537600 \\
-0.24050000 \\
0.04494800 \\
0.27551400 \\
0.17829700 \\
0.01951700 \\
0.74575400 \\
-0.98170300
\end{array}
$$




$\begin{array}{lrrr}\mathrm{H} & -6.52926000 & -0.12726500 & 0.17407400 \\ \mathrm{H} & -4.88457500 & -1.72362100 & 0.40253100 \\ \mathrm{C} & -1.50133700 & 3.11013400 & -0.72275300 \\ \mathrm{H} & -2.57316900 & 3.02376900 & -0.56276700 \\ \mathrm{H} & -1.07124100 & 3.80063400 & 0.00861200 \\ \mathrm{H} & -1.31429600 & 3.52667900 & -1.71648300 \\ \mathrm{C} & 4.99702700 & -0.59516400 & -0.75226900 \\ \mathrm{H} & 5.01280900 & 0.22851400 & -0.03572900 \\ \mathrm{H} & 5.93725300 & -1.13899400 & -0.69863500 \\ \mathrm{H} & 4.87764000 & -0.17855400 & -1.75379500 \\ \mathrm{C} & 0.63701200 & 1.66599000 & -0.63367900 \\ \mathrm{C} & 1.49999200 & 2.72886000 & -1.22599400 \\ \mathrm{H} & 1.06188300 & 3.71429100 & -1.07799600 \\ \mathrm{H} & 2.48271100 & 2.69055300 & -0.75529900 \\ \mathrm{H} & 1.62544900 & 2.54560700 & -2.29753200 \\ \mathrm{O} & 0.81822500 & 2.26297800 & 1.28275300 \\ \mathrm{O} & 2.09335700 & 1.99276800 & 1.65453500 \\ \mathrm{C} & 2.10684500 & 0.85503500 & 2.51381300 \\ \mathrm{H} & 1.58824600 & 0.01946700 & 2.03624000 \\ \mathrm{H} & 3.15658200 & 0.60896700 & 2.67281200 \\ \mathrm{H} & 1.62372700 & 1.10092100 & 3.46149900 \\ \mathrm{~N} & 3.91529700 & -1.51783100 & -0.44459500 \\ \mathrm{H} & 4.13073900 & -2.48516600 & -0.24312600 \\ \mathrm{H} & 3.12862600 & 0.63302600 & 2.70154000 \\ \mathrm{H} & 1.57166300 & 1.12705500 & 3.44257400 \\ \mathrm{~N} & 3.91570500 & -1.51925100 & -0.44381300 \\ \mathrm{H} & 4.12661400 & -2.48669300 & -0.24520600 \\ & & & \end{array}$

\section{Peroxyl-adduct derived from CuATSM (THF)}<smiles>CNC1=NN2C(C)=C(C)[C@](C)(OC)N2N1</smiles>

MN15/CBSB7+ Enthalpy= MN15/CBSB7+ Free Energy=
$-3268.114831$ $-3268.194076$
03
Cu
S
C
N
N
C
N
N
C
S
N
C
H
H
H
H
C
H
H
H
C
H
H

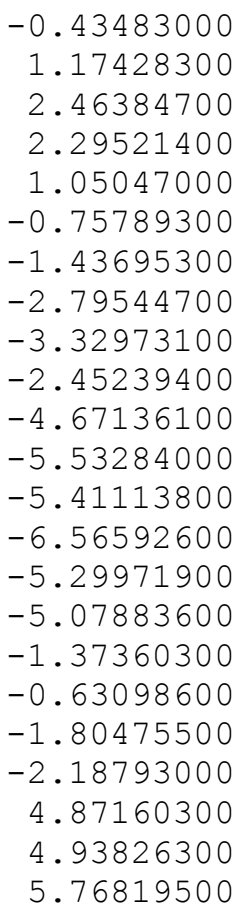

$-0.43483000$

$-0.99811900$

$-2.59738300$

$-1.49204600$

$-0.16758000$

0.20872100

1. 77629800

0.69867200

0.74643200

$-0.44078900$

$-1.94200200$

$-0.51502700$

0.64527800

1.10984000

0.32442100

1.39270200

$-1.42495400$

3. 12476700

3.90685200

3.23155500

3.24005800

$-1.09155600$

$-0.31760300$

$-1.70630900$
0.04230800

0.11254000

$-0.21030900$

$-0.39371300$

$-0.25372200$

$-0.33961200$

$-0.15775100$

$-0.11124000$

0.10457600

0.29921300

0.18397300

0.04921000

$-0.93193600$

0.16683200

0.81088600

0.34401300

$-0.48807500$

$-0.33579900$

$-1.48862600$

0.22855400

$-0.52486800$

0.24253200

$-0.49533900$ 


$\begin{array}{lrrr}\mathrm{H} & 4.79911800 & -0.60562200 & -1.49936600 \\ \mathrm{C} & 0.75635800 & 1.62374200 & -0.40207200 \\ \mathrm{C} & 1.33232600 & 2.19690500 & -1.68838300 \\ \mathrm{H} & 1.12879400 & 3.26710200 & -1.74625800 \\ \mathrm{H} & 2.40753700 & 2.02945900 & -1.70912600 \\ \mathrm{H} & 0.87346800 & 1.69404200 & -2.54159500 \\ \mathrm{O} & 1.17247000 & 2.36360400 & 0.74999400 \\ \mathrm{O} & 2.58261400 & 2.58267300 & 0.70494300 \\ \mathrm{C} & 3.11516500 & 2.00036000 & 1.88196600 \\ \mathrm{H} & 2.93276200 & 0.92350200 & 1.89932600 \\ \mathrm{H} & 4.18714500 & 2.19557300 & 1.83176200 \\ \mathrm{H} & 2.69517400 & 2.47436600 & 2.77210900 \\ \mathrm{~N} & 3.72036100 & -1.94903200 & -0.28701500 \\ \mathrm{H} & 3.86060100 & -2.94265300 & -0.16079500\end{array}$

\section{Peroxyl-adduct derived from CuATSM (Singlet, THF)}<smiles>CNC1=NN2N(N=C(C)[C@@]2(C)OC)N1</smiles>

\section{MN15/CBSB7+ Enthalpy= MN15/CBSB7+ Free Energy=}

\footnotetext{
01

$\mathrm{Cu}$

$\mathrm{S}$

C

$\mathrm{N}$

$\mathrm{N}$

C

$\mathrm{N}$

$\mathrm{N}$

C

S

$\mathrm{N}$

C

$\mathrm{H}$

$\mathrm{H}$

$\mathrm{H}$

$\mathrm{H}$

C

$\mathrm{H}$

$\mathrm{H}$

$\mathrm{H}$

C

$\mathrm{H}$

$\mathrm{H}$

$\mathrm{H}$

C

C

$\mathrm{H}$

$\mathrm{H}$

$\mathrm{H}$

o

O

C

$\mathrm{H}$

$\mathrm{H}$
}

\begin{abstract}
$-0.43657100$
1.11802400

2. 43454800

2.26454300

1.08549400

$-0.73768000$

$-1.41801200$

$-2.77820800$

$-3.32010600$

$-2.45086700$

$-4.66351100$

$-5.51733900$

$-5.37753900$

$-6.55371300$

$-5.29313100$

$-5.07709000$

$-1.35816000$

$-0.60003200$

$-1.92891000$

$-2.06583200$

4.85464600

4.84603300

5.75064200

4.86605900

0.77278500

1.31421200

1.02110800

2.39939100

0.90435300

1. 21775600

2.63940400

3. 11556100

2. 84781700

4.19982100

2.72594700
\end{abstract}

$-3268.114345$

$-3268.192332$

$$
\begin{array}{rr}
-0.99056300 & 0.08397000 \\
-2.62616500 & -0.09214200 \\
-1.52678600 & -0.25275800 \\
-0.19615900 & -0.40541500 \\
0.22420800 & -0.03012100 \\
1.77166200 & -0.33582900 \\
0.68876600 & -0.20024700 \\
0.74140300 & -0.18001400 \\
-0.42145900 & 0.12828400 \\
-1.89929500 & 0.47596100 \\
-0.48523400 & 0.19128900 \\
0.66287300 & -0.05181600 \\
1.04553000 & -1.06535700 \\
0.35614000 & 0.07408700 \\
1.46933200 & 0.65015200 \\
-1.37581600 & 0.42657600 \\
3.11979500 & -0.46701600 \\
3.90101300 & -0.45189800 \\
3.17927800 & -1.39832100 \\
3.27908700 & 0.34962500 \\
-1.10563500 & -0.34134400 \\
-0.39617300 & 0.48825300 \\
-1.71900600 & -0.27945300 \\
-0.54331100 & -1.27714000 \\
1.61032100 & -0.38920700 \\
2.01966200 & -1.74991400 \\
3.04754800 & -1.96993600 \\
1.93942300 & -1.75348600 \\
1.35584400 & -2.51358100 \\
2.48725100 & 0.64857100 \\
2.58802000 & 0.60764300 \\
2.06779700 & 1.83780100 \\
1.01410900 & 1.94457800 \\
2.17497000 & 1.78724400 \\
2.64652900 & 2.67823500
\end{array}
$$


TS structure for C-H HAT of CuATSM (THF)

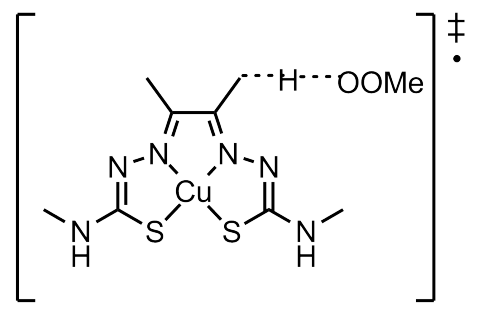

\section{MN15/CBSB7+ Enthalpy $=\quad-3268.074924$ \\ MN15/CBSB7+ Free Energy= $\quad-3268.155237$ Imaginary frequency: -1945.77}

$\begin{array}{lrrr}\text { O 3 } & & & \\ \mathrm{Cu} & -0.27420900 & -1.04120700 & -0.09367100 \\ \mathrm{~S} & -2.20980200 & -2.05587000 & 0.39901600 \\ \mathrm{C} & -3.19317800 & -0.64565200 & 0.05646300 \\ \mathrm{~N} & -2.74634400 & 0.52629400 & -0.35106100 \\ \mathrm{~N} & -1.39886000 & 0.53657000 & -0.49632200 \\ \mathrm{C} & -0.75229100 & 1.60905600 & -0.82134300 \\ \mathrm{C} & 0.71628500 & 1.44817700 & -0.93116800 \\ \mathrm{~N} & 1.14388900 & 0.22872400 & -0.61120600 \\ \mathrm{~N} & 2.43568500 & -0.06772900 & -0.61529700 \\ \mathrm{C} & 2.66716200 & -1.32502900 & -0.20595400 \\ \mathrm{~S} & 1.44754100 & -2.46143800 & 0.27569400 \\ \mathrm{~N} & 3.94547000 & -1.71276900 & -0.16582800 \\ \mathrm{C} & 5.05858900 & -0.84699500 & -0.52421200 \\ \mathrm{H} & 5.08412200 & 0.03365100 & 0.12031400 \\ \mathrm{H} & 5.98178300 & -1.40886900 & -0.40345300 \\ \mathrm{H} & 4.97054100 & -0.51497200 & -1.55974400 \\ \mathrm{H} & 4.13319800 & -2.65612400 & 0.14710400 \\ \mathrm{C} & 1.57973500 & 2.54068600 & -1.25094400 \\ \mathrm{H} & 1.14304000 & 3.31867600 & -1.87301900 \\ \mathrm{H} & 2.59324500 & 2.25960800 & -1.52096400 \\ \mathrm{C} & -1.37845300 & 2.94362300 & -1.03140400 \\ \mathrm{H} & -1.29026800 & 3.25074200 & -2.07751500 \\ \mathrm{H} & -2.42992000 & 2.91422900 & -0.75647100 \\ \mathrm{H} & -0.85536200 & 3.68652000 & -0.42171400 \\ \mathrm{~N} & -4.51953900 & -0.78418000 & 0.23407800 \\ \mathrm{C} & -5.46139500 & 0.29423000 & -0.00546300 \\ \mathrm{H} & -5.42415200 & 0.61956000 & -1.04748300 \\ \mathrm{H} & -6.46313900 & -0.06438800 & 0.22171800 \\ \mathrm{H} & -5.23533000 & 1.15443900 & 0.62849700 \\ \mathrm{H} & -4.85708000 & -1.68277100 & 0.54798100 \\ \mathrm{H} & 1.67181300 & 3.13276100 & -0.13170500 \\ \mathrm{O} & 1.49119700 & 3.52824000 & 1.11957100 \\ \mathrm{O} & 0.77620100 & 2.46754600 & 1.59917700 \\ \mathrm{C} & 1.65856100 & 1.55211900 & 2.23966800 \\ \mathrm{H} & 2.07209800 & 2.00355700 & 3.14317900 \\ \mathrm{H} & 1.05849300 & 0.67515600 & 2.48753500 \\ \mathrm{H} & 2.46376500 & 1.27421200 & 1.55245600\end{array}$

\section{Methylene derived from CuATSM (THF)}


<smiles></smiles>

\section{MN15/CBSB7+ Enthalpy $=\quad-3077.443143$ \\ MN15/CBSB7+ Free Energy= $\quad-3077.512412$}

$\begin{array}{lrrr}03 & & \\ \mathrm{Cu} & 0.02828100 & -0.66910500 & 0.00000300 \\ \mathrm{~S} & 1.90674200 & -1.93436800 & 0.00000100 \\ \mathrm{C} & 2.98411800 & -0.57577000 & -0.00000200 \\ \mathrm{~N} & 2.58422000 & 0.77823500 & -0.00000300 \\ \mathrm{~N} & 1.27730400 & 0.83145200 & -0.00000100 \\ \mathrm{C} & 0.70473800 & 2.09896200 & 0.00000000 \\ \mathrm{C} & -0.77585600 & 2.02327600 & -0.00000100 \\ \mathrm{~N} & -1.26245300 & 0.82344800 & 0.00000300 \\ \mathrm{~N} & -2.60632300 & 0.62742000 & 0.00000500 \\ \mathrm{C} & -2.92277700 & -0.65301500 & 0.00001100 \\ \mathrm{~S} & -1.78928300 & -1.98758500 & 0.00000800 \\ \mathrm{~N} & -4.23061700 & -0.97377300 & -0.00000400 \\ \mathrm{C} & -5.28263000 & 0.02578100 & -0.00001300 \\ \mathrm{H} & -5.21391100 & 0.66328500 & -0.88446500 \\ \mathrm{H} & -6.24418400 & -0.48335900 & -0.00002500 \\ \mathrm{H} & -5.21393000 & 0.66328000 & 0.88444300 \\ \mathrm{H} & -4.47118000 & -1.95448700 & -0.00001000 \\ \mathrm{C} & -1.66581100 & 3.21975900 & -0.00000500 \\ \mathrm{H} & -1.11001000 & 4.15359700 & -0.00002700 \\ \mathrm{H} & -2.31772600 & 3.18881800 & -0.87646400 \\ \mathrm{H} & -2.31770000 & 3.18884400 & 0.87647500 \\ \mathrm{C} & 5.35302100 & 0.18404200 & -0.00000900 \\ \mathrm{H} & 4.90015700 & 1.17128300 & 0.00001300 \\ \mathrm{H} & 5.97682400 & 0.07385300 & 0.88835600 \\ \mathrm{H} & 5.97679300 & 0.07388000 & -0.88840000 \\ \mathrm{C} & 1.41887200 & 3.24602200 & 0.00000300 \\ \mathrm{H} & 2.49956400 & 3.20481300 & 0.00000400 \\ \mathrm{H} & 0.94140200 & 4.21469400 & 0.00000400 \\ \mathrm{~N} & 4.30061600 & -0.81995200 & -0.00000400 \\ \mathrm{H} & 4.57313600 & -1.79536600 & -0.00000700 \\ & & & \end{array}$

$N$-methylimine derived from methylperoxyl-substituted CuATSM (THF)<smiles>CN=C1NN2[C@](C)(OC)C(C)=NN3N=C(NC)S[C@@]32S1</smiles>

$\begin{array}{lr}\text { MN15/CBSB7+ Enthalpy }= & -3267.495263 \\ \text { MN15/CBSB7+ Free Energy }= & -3267.574360\end{array}$

$\begin{array}{lrrr}02 & & & \\ \mathrm{Cu} & -0.34726400 & -0.96225400 & 0.10937900 \\ \mathrm{~S} & 1.28381400 & -2.49369000 & 0.18321600 \\ \mathrm{C} & 2.57327900 & -1.43952600 & -0.37664500 \\ \mathrm{~N} & 2.29250900 & -0.02799300 & -0.57413800 \\ \mathrm{~N} & 1.13161600 & 0.28097900 & -0.31036400 \\ \mathrm{C} & -0.75503300 & 1.79107200 & -0.45090700 \\ \mathrm{~N} & -1.39658200 & 0.69786000 & -0.21956900 \\ \mathrm{~N} & -2.75281400 & 0.67874000 & -0.24435000 \\ \mathrm{C} & -3.24769600 & -0.51204200 & 0.04446400\end{array}$


$-1.95026200$

$-0.63943000$

0.46442100

0.85234400

0.10458700

1.28054600

$-1.55028000$

3.10876200

3.90046100

3. 33027900

3.09381500

$-1.02047300$

0.02462400

$-1.08158400$

$-1.43697100$

1.71914000

2.49499100

3.54941500

2. 38361000

2.12079400

2.20775800

2.28103800

1.50934000

0.46627100

1.57710600

1.92795900

$-1.86713700$
0.41271700

0.05981700

$-0.22260000$

$-1.23013700$

$-0.14040800$

0.48687900

0.27535300

$-0.73548900$

$-0.20291300$

$-1.80604600$

$-0.43807700$

$-1.05586100$

$-1.20927900$

$-0.32903800$

$-1.98943600$

$-0.34662200$

$-1.41465600$

$-1.34185500$

$-1.27874100$

$-2.40101200$

0.96730800

1.19629800

2.36383800

2. 21189100

2.52226500

3.22044200

$-0.59693600$

\section{di-(methylperoxyl) adduct derived from CuATSM (THF)}<smiles>CNC1=NN2C(OC)C(NC)=NN3C(C)=C(OC)N(C)C32S1</smiles>

\section{MN15/CBSB7+ Enthalpy $=\quad-3458.171200$ \\ MN15/CBSB7+ Free Energy $=\quad-3458.257078$}

$\begin{array}{ll}0 & 2 \\ S & \\ N \\ N \\ C \\ N \\ N \\ N \\ C \\ S \\ N \\ \text { C } \\ H \\ H \\ H \\ \text { C } \\ H \\ H \\ H \\ \text { C } \\ \text { C } \\ \text { H } \\ \text { H } \\ \text { H } \\ \text { O }\end{array}$

$$
\begin{array}{r}
-1.41768900 \\
-2.08766100 \\
-0.93008600 \\
1.09003100 \\
1.61303700 \\
2.95445300 \\
3.32888200 \\
2.27589700 \\
4.64221200 \\
5.64057000 \\
5.45350400 \\
6.62007300 \\
5.63009700 \\
1.85543800 \\
1.44584800 \\
1.77168400 \\
2.90630800 \\
-0.42058200 \\
-1.10924900 \\
-0.72518200 \\
-2.18238700 \\
-0.91379100 \\
-0.55374700
\end{array}
$$

-1.36086100
0.44802300
0.19029600
0.68460100
0.23614500
0.30409800
-0.23819600
-0.97337800
-0.22986500
0.34091000
1.40444700
0.21599800
-0.16036000
1.26910600
0.91328500
2.36056100
0.99964000
0.53958900
1.73913300
1.90013400
1.56602000
2.62535200
-0.64173100 


$\begin{array}{lrrr}\mathrm{O} & -1.94351500 & 3.03864100 & -0.86980800 \\ \mathrm{C} & -2.23675800 & 2.50158000 & -2.15306000 \\ \mathrm{H} & -2.05563000 & 1.42282400 & -2.17742200 \\ \mathrm{H} & -3.29702400 & 2.70574600 & -2.30263700 \\ \mathrm{H} & -1.64792800 & 3.00353500 & -2.92301700 \\ \mathrm{Cu} & 0.41053400 & -0.59220700 & -0.55546300 \\ \mathrm{C} & -2.41376800 & -1.03543200 & 0.01251500 \\ \mathrm{O} & -2.21239500 & -1.78341700 & 1.22512600 \\ \mathrm{O} & -0.82893300 & -1.74314300 & 1.57774100 \\ \mathrm{C} & -0.37719600 & -3.09184400 & 1.61863600 \\ \mathrm{H} & -0.47667400 & -3.55188000 & 0.63369400 \\ \mathrm{H} & 0.67520500 & -3.02408200 & 1.89667700 \\ \mathrm{H} & -0.93160700 & -3.65372700 & 2.37251000 \\ \mathrm{~N} & -3.78910700 & -1.05733600 & -0.29893100 \\ \mathrm{C} & -4.74732400 & -0.86125200 & 0.79005300 \\ \mathrm{H} & -4.45779000 & 0.01945300 & 1.36376800 \\ \mathrm{H} & -5.73106600 & -0.67826100 & 0.35841500 \\ \mathrm{H} & -4.80959400 & -1.71471200 & 1.47099900 \\ \mathrm{H} & -4.01218100 & -1.83096000 & -0.91627000 \\ \mathrm{H} & 4.92984100 & -1.71534800 & -0.64425000\end{array}$

Peroxyl adduct from addition at thiyl-iminyl carbon of CuATSM (THF)<smiles>CNC1=NN2C(C)=C(C)N3[C@]2(OC)SC(S1)[N+]3(NC)OC</smiles>

\section{$\mathrm{MN15} / \mathrm{CBSB} 7+$ Enthalpy $=\quad-3268.086150$ \\ MN15/CBSB7+ Free Energy= $\quad-3268.165923$}

$\begin{array}{lrrr}03 & & \\ \mathrm{Cu} & 0.48929100 & -0.67976900 & 0.03832900 \\ \mathrm{~S} & 2.27598800 & -2.03052900 & -0.27154200 \\ \mathrm{C} & 3.43049300 & -0.74336500 & -0.19450000 \\ \mathrm{~N} & 3.12843500 & 0.55617400 & -0.00667900 \\ \mathrm{~N} & 1.83014300 & 0.75474500 & 0.11437400 \\ \mathrm{C} & 1.35713900 & 1.99961900 & 0.30990400 \\ \mathrm{C} & -0.04061700 & 2.10022500 & 0.43080200 \\ \mathrm{~N} & -0.70052200 & 0.89403500 & 0.32058100 \\ \mathrm{~N} & -1.95023000 & 0.85145700 & 0.41900300 \\ \mathrm{~S} & -1.37395900 & -1.87996300 & 0.08640300 \\ \mathrm{C} & -0.83898300 & 3.33381100 & 0.65868800 \\ \mathrm{H} & -0.21522200 & 4.22017800 & 0.73546800 \\ \mathrm{H} & -1.55437800 & 3.47321900 & -0.15753700 \\ \mathrm{H} & -1.42845900 & 3.23193900 & 1.57439100 \\ \mathrm{C} & 2.31360300 & 3.14178100 & 0.38132000 \\ \mathrm{H} & 3.02603000 & 2.97113600 & 1.19209800 \\ \mathrm{H} & 2.89536500 & 3.18881000 & -0.54242000 \\ \mathrm{H} & 1.81316100 & 4.09322600 & 0.53809400 \\ \mathrm{C} & 5.78746200 & -0.04044000 & -0.28095400 \\ \mathrm{H} & 5.65598700 & 0.70057600 & -1.07088200 \\ \mathrm{H} & 5.78958700 & 0.47486200 & 0.68079000 \\ \mathrm{H} & 6.73872300 & -0.54871400 & -0.41882800 \\ \mathrm{C} & -2.52884200 & -0.49034200 & 0.23302500 \\ \mathrm{O} & -3.15388000 & -0.46155700 & -1.07156700 \\ \mathrm{O} & -4.26212000 & 0.44966400 & -1.02337600 \\ \mathrm{C} & -3.97511900 & 1.48954100 & -1.94081800 \\ \mathrm{H} & -3.82799700 & 1.08897000 & -2.94622600 \\ \mathrm{H} & -4.85346300 & 2.13573600 & -1.92618500 \\ \mathrm{H} & -3.09232400 & 2.05202100 & -1.62487100 \\ \mathrm{~N} & -3.45032800 & -0.63484500 & 1.30536000 \\ \mathrm{H} & & & \end{array}$




$\begin{array}{rrrr}\mathrm{H} & -4.01131600 & 0.21369300 & 1.35295900 \\ \mathrm{C} & -4.29672600 & -1.82465000 & 1.25870000 \\ \mathrm{H} & -3.68145300 & -2.71753500 & 1.37806700 \\ \mathrm{H} & -5.00242800 & -1.78293700 & 2.08805300 \\ \mathrm{H} & -4.85598100 & -1.90679000 & 0.31925500 \\ \mathrm{~N} & 4.72689300 & -1.03599100 & -0.32842700 \\ \mathrm{H} & 4.96906600 & -2.00818800 & -0.46849100\end{array}$

\section{$N$-methylimine derived from peroxyl-substituted CuATSM (THF)}<smiles>CN=C1NN2C(C)=C(C)N3NC(NC)(S1)SC23OC</smiles>

\section{MN15/CBSB7+ Enthalpy $=\quad-3267.454507$ \\ $\mathrm{MN15/CBSB7+Free} \mathrm{Energy}=\quad-3267.533073$}

$\begin{array}{lrrr}02 & & \\ \mathrm{Cu} & 0.52945300 & -0.72393600 & -0.02995800 \\ \mathrm{~S} & 2.29540000 & -2.04804900 & -0.29940700 \\ \mathrm{C} & 3.51720500 & -0.78507900 & -0.18234400 \\ \mathrm{~N} & 3.11241400 & 0.58285000 & 0.01450400 \\ \mathrm{~N} & 1.88276100 & 0.73460000 & 0.09793400 \\ \mathrm{C} & 1.37416700 & 2.04319300 & 0.28896900 \\ \mathrm{C} & 0.01906300 & 2.11531600 & 0.37201100 \\ \mathrm{~N} & -0.64616900 & 0.86160800 & 0.25788100 \\ \mathrm{~N} & -1.87767100 & 0.84234300 & 0.32411100 \\ \mathrm{~S} & -1.36093900 & -1.88710700 & -0.11892700 \\ \mathrm{C} & -0.81536100 & 3.32908200 & 0.56538900 \\ \mathrm{H} & -0.21125300 & 4.22806300 & 0.64779900 \\ \mathrm{H} & -1.50809000 & 3.43916100 & -0.27335900 \\ \mathrm{H} & -1.42376900 & 3.21989900 & 1.46637000 \\ \mathrm{C} & 2.34779900 & 3.16375100 & 0.37457900 \\ \mathrm{H} & 3.03989300 & 2.98852600 & 1.20167600 \\ \mathrm{H} & 2.94658400 & 3.20337700 & -0.53846300 \\ \mathrm{H} & 1.85332700 & 4.12009500 & 0.51890600 \\ \mathrm{~N} & 4.75508400 & -1.05850100 & -0.27049000 \\ \mathrm{C} & 5.79412000 & -0.05493800 & -0.18025000 \\ \mathrm{H} & 5.43194100 & 0.96415000 & -0.03577800 \\ \mathrm{H} & 6.45928500 & -0.32507100 & 0.64383200 \\ \mathrm{H} & 6.39526100 & -0.10260700 & -1.09169600 \\ \mathrm{C} & -2.48527100 & -0.50710600 & 0.18876200 \\ \mathrm{O} & -3.25347400 & -0.43274500 & -1.02552800 \\ \mathrm{O} & -4.35735400 & 0.45923000 & -0.81295900 \\ \mathrm{C} & -4.20805200 & 1.51836800 & -1.74229300 \\ \mathrm{H} & -4.19194600 & 1.13631500 & -2.76494600 \\ \mathrm{H} & -5.08434200 & 2.15064000 & -1.59720100 \\ \mathrm{H} & -3.29760700 & 2.08788700 & -1.53622500 \\ \mathrm{~N} & -3.25931400 & -0.63894600 & 1.37005900 \\ \mathrm{H} & -3.85276900 & 0.18447000 & 1.45513400 \\ \mathrm{C} & -4.04520100 & -1.86697100 & 1.48593000 \\ \mathrm{H} & -3.37875300 & -2.72160000 & 1.60304900 \\ \mathrm{H} & -4.67038500 & -1.79337400 & 2.37513400 \\ \mathrm{H} & -4.68679400 & -2.03655200 & 0.61330400 \\ & & & \\ & & & \\ & & & \\ & & & \end{array}$




\section{CuGTSM (THF)}<smiles>CNC1=NN2C=CN3N=C(NC)SC23S1</smiles>

\section{MN15/CBSB7+ Enthalpy $=\quad-2999.564576$ \\ $\mathrm{MN15/CBSB7+Free} \mathrm{Energy}=\quad-2999.627795$}

$\begin{array}{lrrr}0 \mathrm{O} & & \\ \mathrm{Cu} & 0.00000000 & 0.33376700 & -0.00000200 \\ \mathrm{~S} & -1.85392900 & 1.61201000 & -0.00000500 \\ \mathrm{C} & -2.96715600 & 0.25998000 & -0.00000200 \\ \mathrm{~N} & -2.62983800 & -1.02211200 & 0.00000500 \\ \mathrm{~N} & -1.29429700 & -1.18188300 & 0.00000400 \\ \mathrm{~N} & 1.29429700 & -1.18188300 & 0.00000400 \\ \mathrm{~N} & 2.62983800 & -1.02211200 & 0.00000200 \\ \mathrm{C} & 2.96715600 & 0.25998000 & -0.00000500 \\ \mathrm{~S} & 1.85392900 & 1.61201000 & -0.00001100 \\ \mathrm{~N} & 4.27538600 & 0.55673900 & -0.00000900 \\ \mathrm{C} & 5.31688200 & -0.45652300 & -0.00000400 \\ \mathrm{H} & 5.23986200 & -1.09124200 & -0.88496500 \\ \mathrm{H} & 6.28320400 & 0.04283300 & -0.00001700 \\ \mathrm{H} & 5.23987600 & -1.09122000 & 0.88497400 \\ \mathrm{H} & 4.53128900 & 1.53424300 & -0.00001100 \\ \mathrm{~N} & -4.27538600 & 0.55673900 & 0.00000500 \\ \mathrm{C} & -5.31688200 & -0.45652300 & 0.00001800 \\ \mathrm{H} & -5.23987200 & -1.09121800 & 0.88499700 \\ \mathrm{H} & -6.28320400 & 0.04283300 & 0.00000800 \\ \mathrm{H} & -5.23986600 & -1.09124400 & -0.88494100 \\ \mathrm{H} & -4.53128900 & 1.53424300 & 0.00001200 \\ \mathrm{C} & -0.73031500 & -2.34279000 & 0.00001000 \\ \mathrm{H} & -1.31142600 & -3.25960300 & 0.00001500 \\ \mathrm{C} & 0.73031500 & -2.34279000 & 0.00000900 \\ \mathrm{H} & 1.31142600 & -3.25960300 & 0.00001200\end{array}$

\section{Aminyl radical derived from CuGTSM (THF)}<smiles>CNC1=NN2C=CN3N=C(NC)SC23S1</smiles>

\section{MN15/CBSB7+ Enthalpy $=\quad-2998.917304$ MN15/CBSB7+ Free Energy= $\quad-2998.980063$}

$\begin{array}{lrrr}03 & & \\ \mathrm{Cu} & 0.03677000 & -0.37595100 & -0.00003800 \\ \mathrm{~S} & 1.86298700 & -1.63383700 & -0.00017600 \\ \mathrm{C} & 3.04384800 & -0.31032700 & -0.00001500 \\ \mathrm{~N} & 2.60312000 & 1.03689900 & 0.00014600 \\ \mathrm{~N} & 1.33852300 & 1.14598600 & 0.00015200 \\ \mathrm{~N} & -1.24112100 & 1.14506600 & 0.00013500 \\ \mathrm{~N} & -2.54581600 & 1.02164200 & 0.00010600 \\ \mathrm{C} & -2.92812200 & -0.28056900 & -0.00005400 \\ \mathrm{~S} & -1.85186100 & -1.63136300 & -0.00020200 \\ \mathrm{~N} & -4.24147000 & -0.49945300 & -0.00010500 \\ \mathrm{C} & -5.24472900 & 0.55764500 & 0.00000400 \\ \mathrm{H} & -5.14148200 & 1.18499100 & -0.88596300 \\ \mathrm{H} & -6.22727600 & 0.09240700 & -0.00007400\end{array}$




$\begin{array}{lrrr}\mathrm{H} & -5.14151900 & 1.18477100 & 0.88613200 \\ \mathrm{H} & -4.54695100 & -1.46458400 & -0.00022900 \\ \mathrm{~N} & 4.28930300 & -0.58633800 & -0.00005200 \\ \mathrm{C} & 5.30439400 & 0.44650500 & 0.00006800 \\ \mathrm{H} & 4.91329600 & 1.46531100 & 0.00019800 \\ \mathrm{H} & 5.94309000 & 0.30325700 & 0.87575400 \\ \mathrm{H} & 5.94307400 & 0.30347600 & -0.87566500 \\ \mathrm{C} & 0.73704000 & 2.36113700 & 0.00029800 \\ \mathrm{H} & 1.33345900 & 3.26485200 & 0.00041300 \\ \mathrm{C} & -0.65881200 & 2.34508100 & 0.00028600 \\ \mathrm{H} & -1.25952800 & 3.24786000 & 0.00039000\end{array}$

TS structure for addition of MeOO• to CuGTSM (THF)<smiles>CNC1=NN2C=C(OC)N3N=C(NC)SC23S1</smiles>

MN15/CBSB7+ Enthalpy $=-3189.581938$ MN15/CBSB7+ Free Energy= $-3189.655274$

Imaginary frequency: -549.92

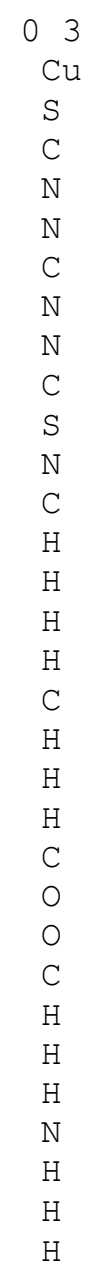

$$
\begin{array}{r}
-0.32354100 \\
1.43253900 \\
2.62291900 \\
2.35853900 \\
1.06679700 \\
-0.83518900 \\
-1.47899700 \\
-2.82031400 \\
-3.25663000 \\
-2.25800100 \\
-4.58032000 \\
-5.53640500 \\
-6.53577100 \\
-5.34062000 \\
-5.48005400 \\
-4.91097600 \\
4.99347400 \\
5.01322500 \\
5.92991100 \\
4.87707500 \\
0.62742700 \\
0.94877200 \\
2.19490400 \\
2.11412600 \\
1.54862100 \\
3.14184200 \\
1.63228600 \\
3.90523800 \\
4.11578700 \\
-1.33984600 \\
1.25427600
\end{array}
$$

$$
\begin{array}{r}
-0.05532800 \\
0.36798700 \\
-0.30302500 \\
-0.84040600 \\
-0.77292100 \\
-1.09159000 \\
-0.60703200 \\
-0.49214500 \\
0.04626100 \\
0.56325200 \\
0.21627900 \\
-0.16295600 \\
0.08203700 \\
0.37542000 \\
-1.23373700 \\
0.62820200 \\
-0.76254600 \\
-0.22815800 \\
-0.59709100 \\
-1.82794400 \\
-1.11055500 \\
0.55897400 \\
0.99967900 \\
2.10238200 \\
1.81881700 \\
2.34864200 \\
2.94858200 \\
-0.27599200 \\
0.12919200 \\
-1.42603300 \\
-1.68475100
\end{array}
$$

Peroxyl adduct derived from CuGTSM (THF) 
<smiles>CNC1=NN2C=C(OC)N3N=C(NC)S[C@]23S1</smiles>

\section{MN15/CBSB7+ Enthalpy $=\quad-3189.614081$ \\ MN15/CBSB7+ Free Energy $=\quad-3189.686895$}

$\begin{array}{lrrr}03 & & \\ \mathrm{Cu} & -0.46441500 & -0.74727700 & 0.06711100 \\ \mathrm{~S} & 1.10820200 & -2.37008200 & 0.29182000 \\ \mathrm{C} & 2.42146100 & -1.34649100 & -0.17430100 \\ \mathrm{~N} & 2.28489500 & -0.04229500 & -0.49238200 \\ \mathrm{~N} & 1.05346600 & 0.37811000 & -0.36386100 \\ \mathrm{~N} & -1.43204300 & 0.94652200 & -0.34898300 \\ \mathrm{~N} & -2.78114600 & 1.03899300 & -0.31965700 \\ \mathrm{C} & -3.34831100 & -0.10142500 & 0.04046900 \\ \mathrm{~S} & -2.51195400 & -1.58707000 & 0.42951700 \\ \mathrm{~N} & -4.68916100 & -0.12876000 & 0.11643000 \\ \mathrm{C} & -5.52196900 & 1.02617300 & -0.17104000 \\ \mathrm{H} & -5.37459800 & 1.36562900 & -1.19832900 \\ \mathrm{H} & -6.56326300 & 0.74227200 & -0.03425400 \\ \mathrm{H} & -5.28390500 & 1.85362100 & 0.50044800 \\ \mathrm{H} & -5.12175500 & -0.99998900 & 0.38848000 \\ \mathrm{C} & 4.82753500 & -1.05744300 & -0.59905400 \\ \mathrm{H} & 4.93652800 & -0.20345800 & 0.07217400 \\ \mathrm{H} & 5.70753700 & -1.69149500 & -0.52200800 \\ \mathrm{H} & 4.73934000 & -0.68441600 & -1.62077600 \\ \mathrm{C} & 0.78961000 & 1.76682100 & -0.66077700 \\ \mathrm{O} & 1.24869300 & 2.64948800 & 0.35087300 \\ \mathrm{O} & 2.63981100 & 2.89092600 & 0.12433800 \\ \mathrm{C} & 3.31668700 & 2.50688700 & 1.30876000 \\ \mathrm{H} & 3.18186500 & 1.44054400 & 1.50333900 \\ \mathrm{H} & 4.36794900 & 2.72023300 & 1.11196500 \\ \mathrm{H} & 2.97327700 & 3.09933300 & 2.15970300 \\ \mathrm{~N} & 3.66052700 & -1.84729000 & -0.23448200 \\ \mathrm{H} & 3.77608700 & -2.82702300 & -0.01078700 \\ \mathrm{C} & -0.70435800 & 1.94875100 & -0.67754900 \\ \mathrm{H} & -1.13768100 & 2.91217700 & -0.92981300 \\ \mathrm{H} & 1.25896200 & 2.05813300 & -1.60794200 \\ & & & \end{array}$

$\mathrm{N}$-methylimine derived from peroxyl-substituted CuGTSM (THF)<smiles></smiles>

$\begin{array}{lr}\text { MN15/CBSB7+ Enthalpy }= & -3188.992978 \\ \text { MN15/CBSB7+ Free Energy= } & -3189.066185\end{array}$

$\begin{array}{lrrr}02 & & \\ \mathrm{Cu} & -0.40333800 & -0.72586500 & 0.14125300 \\ \mathrm{~S} & 1.15820400 & -2.30960400 & 0.37265000 \\ \mathrm{C} & 2.50577800 & -1.36760800 & -0.25024200 \\ \mathrm{~N} & 2.27482800 & 0.00714900 & -0.65997600 \\ \mathrm{~N} & 1.13062600 & 0.39586800 & -0.42750500 \\ \mathrm{C} & -0.68638000 & 1.91248000 & -0.83550600 \\ \mathrm{~N} & -1.38843900 & 0.92245600 & -0.41645600 \\ \mathrm{~N} & -2.73430000 & 0.97834100 & -0.43122700\end{array}$


$-0.13796900$

$-1.55567700$

$-0.18695000$

0.91449400

1.15371300

0.61965800

1.80852700

$-1.03683900$

$-1.84805000$

$-1.09743000$

$-0.08124700$

$-1.06518200$

$-1.65396300$

1.80609200

2.59698200

2.77245500

2.38285300

1.33030200

2. 53747100

3.01148100

2. 82519900

2.13079400
0.02761400

0.58162100

0.07083100

$-0.35359300$

$-1.40454000$

$-0.21921100$

0.23990100

0.41549000

$-0.34705500$

$-0.85421700$

$-1.17402000$

$-0.07441000$

$-1.68941200$

$-0.73052800$

0.37200300

0.32249700

1.59471600

1.79276100

1. 53145400

2. 38138400

$-1.20489700$

$-1.62493600$

\section{$\mathrm{CuATSM}_{2}$ (THF)}

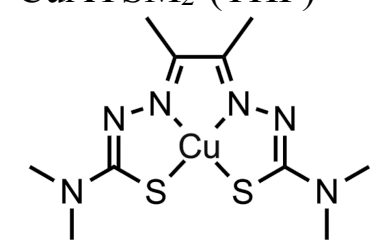

\section{MN15/CBSB7+ Enthalpy $=-3156.525666$ \\ $\mathrm{MN15/CBSB7+Free} \mathrm{Energy=} \quad-3156.606744$}

02

Cu
S
$C$
N
N
C
C
N
N
C
S
N
C
H
H
H
C
H
H
H
C
H
H
H
N
C
H
H

\begin{abstract}
0.00681800
1.85008000

2.97394200

2.62649300

1.28713500

0.73659700

$-0.74566500$

$-1.27967600$

$-2.61713600$

$-2.96573900$

$-1.83941200$

$-4.28436000$

$-5.22942900$

$-5.07682200$

$-6.24042400$

$-5.10225300$

$-1.57686600$

$-0.96929400$

$-2.23104800$

$-2.22980600$

1.50559400

1.26065100

2. 57271100

1. 24882300

4.29480600

5.23915600

5.14366100

6.24901500
\end{abstract}
$-0.48675100$
$-1.77121800$
$-0.42547200$
0.85505100
1.02745000
2.20086700
2.18905800
1.00847500
0.84467300
$-0.43915700$
$-1.78056600$
$-0.71351400$
0.39820100
1.05317700
$-0.00237400$
0.99661200
3.42762500
4.33035300
3.43703500
3.42925400
3.47923000
4.07187600
3.27017600
4.08128100
$-0.70063700$
0.40961300
0.95435300
0.01366700

$-0.00830200$

$-0.01646900$

$-0.02137600$

$-0.00816500$

$-0.00770000$

$-0.00311700$

0.00052400

$-0.00183300$

0.00116200

$-0.00425600$

$-0.00823700$

$-0.00477100$

0.02078900

$-0.83919300$

$-0.00661500$

0.92593900

0.00631400

0.00987700

$-0.86980200$

0.88337200

$-0.00154200$

0.88424100

$-0.00956300$

$-0.87750300$

$-0.03423900$

0.02990100

0.97301200

$-0.05535300$ 


$\begin{array}{lrrr}\text { H } & 5.05750400 & 1.11266400 & -0.78427800 \\ \mathrm{C} & 4.79141700 & -2.06805200 & 0.04411100 \\ \mathrm{H} & 4.58689700 & -2.51776900 & 1.02001800 \\ \mathrm{H} & 4.33484400 & -2.69212200 & -0.72531200 \\ \mathrm{H} & 5.86778200 & -2.05263900 & -0.11619400 \\ \mathrm{C} & -4.77711600 & -2.08484700 & 0.01893700 \\ \mathrm{H} & -4.40254500 & -2.65244600 & -0.83534200 \\ \mathrm{H} & -4.47642800 & -2.60101800 & 0.93407600 \\ \mathrm{H} & -5.86383900 & -2.06064600 & -0.02821000\end{array}$

\section{Peroxyl adduct derived from $\mathrm{CuATSM}_{2}$ (THF)}<smiles></smiles>

\section{MN15/CBSB7+ Enthalpy $=\quad-3346.577084$ MN15/CBSB7+ Free Energy= $\quad-3346.664818$}

\begin{tabular}{|c|c|c|c|}
\hline \multicolumn{4}{|l|}{03} \\
\hline $\mathrm{S}$ & 1.31282300 & -2.34838600 & 0.10608300 \\
\hline $\mathrm{C}$ & 2.57584200 & -1.20086500 & -0.18445200 \\
\hline $\mathrm{N}$ & 2.35110500 & 0.11914700 & -0.36613600 \\
\hline $\mathrm{N}$ & 1.09225800 & 0.44810200 & -0.24343100 \\
\hline C & -0.76969200 & 1.94843500 & -0.35395200 \\
\hline $\mathrm{N}$ & -1.41442700 & 0.84539000 & -0.19115600 \\
\hline $\mathrm{N}$ & -2.76985600 & 0.84635100 & -0.16308200 \\
\hline $\mathrm{C}$ & -3.27848400 & -0.36405500 & 0.02889600 \\
\hline $\mathrm{S}$ & -2.33844100 & -1.82535000 & 0.23749500 \\
\hline $\mathrm{N}$ & -4.62390300 & -0.46021400 & 0.06883800 \\
\hline $\mathrm{C}$ & -5.41233500 & 0.76205300 & -0.04149900 \\
\hline $\mathrm{H}$ & -5.17152700 & 1.29083400 & -0.96525300 \\
\hline $\mathrm{H}$ & -6.46781500 & 0.49766200 & -0.04230400 \\
\hline $\mathrm{H}$ & -5.20995400 & 1.43765200 & 0.79394900 \\
\hline $\mathrm{C}$ & -1.43651500 & 3.27301200 & -0.49714100 \\
\hline $\mathrm{H}$ & -0.72054500 & 4.08384500 & -0.37041700 \\
\hline $\mathrm{H}$ & -1.90227300 & 3.35529300 & -1.48443500 \\
\hline $\mathrm{H}$ & -2.23458900 & 3.36474100 & 0.24188500 \\
\hline $\mathrm{C}$ & 4.90790000 & -0.60939400 & -0.48412200 \\
\hline $\mathrm{H}$ & 4.83090200 & 0.21032000 & 0.23154500 \\
\hline $\mathrm{H}$ & 5.87328600 & -1.09747300 & -0.37211700 \\
\hline $\mathrm{H}$ & 4.82776700 & -0.19338200 & -1.49065000 \\
\hline $\mathrm{C}$ & 0.74916000 & 1.85093300 & -0.39316100 \\
\hline $\mathrm{C}$ & 1.32641800 & 2.45005400 & -1.66732700 \\
\hline $\mathrm{H}$ & 1.08086000 & 3.51122100 & -1.72908200 \\
\hline $\mathrm{H}$ & 2.40787300 & 2.32656700 & -1.66729600 \\
\hline $\mathrm{H}$ & 0.90552200 & 1.92996800 & -2.52975100 \\
\hline 0 & 1.11928500 & 2.60621200 & 0.76691300 \\
\hline 0 & 2.52283700 & 2.86920400 & 0.75074100 \\
\hline $\mathrm{C}$ & 3.04942600 & 2.29776800 & 1.93531600 \\
\hline $\mathrm{H}$ & 2.90095000 & 1.21543500 & 1.94377900 \\
\hline $\mathrm{H}$ & 4.11553500 & 2.52740200 & 1.90916500 \\
\hline $\mathrm{H}$ & 2.59624400 & 2.75280700 & 2.81917100 \\
\hline $\mathrm{N}$ & 3.85735500 & -1.59748000 & -0.24206200 \\
\hline $\mathrm{Cu}$ & -0.35195100 & -0.80838100 & 0.01890000 \\
\hline $\mathrm{C}$ & -5.29031300 & -1.72501400 & 0.34785900 \\
\hline $\mathrm{H}$ & -4.95821700 & -2.50171200 & -0.34301600 \\
\hline $\mathrm{H}$ & -5.09811500 & -2.06688800 & 1.36886800 \\
\hline $\mathrm{H}$ & -6.36204500 & -1.58410600 & 0.22169400 \\
\hline
\end{tabular}




$\begin{array}{lrrr}\mathrm{C} & 4.20130000 & -3.00678500 & -0.11179500 \\ \mathrm{H} & 3.86725400 & -3.39941000 & 0.85138700 \\ \mathrm{H} & 3.73296900 & -3.59690300 & -0.90332300 \\ \mathrm{H} & 5.28144600 & -3.11030300 & -0.18291000\end{array}$

TS structure for addition of $\mathrm{MeOO} \bullet$ to $\mathrm{CuATSM}_{2}$ (THF)<smiles></smiles>

\section{MN15/CBSB7+ Enthalpy $=-3346.548226$ \\ MN15/CBSB7+ Free Energy $=\quad-3346.635757$ Imaginary Frequency= $-484.34$}

$\begin{array}{lrrr}\text { O } 3 & & \\ \mathrm{Cu} & -0.27199200 & -0.72740300 & -0.14198900 \\ \mathrm{~S} & 1.50220000 & -2.13413100 & 0.00098300 \\ \mathrm{C} & 2.68520100 & -0.92172200 & -0.38197200 \\ \mathrm{~N} & 2.39140100 & 0.36710800 & -0.64279700 \\ \mathrm{~N} & 1.09713600 & 0.62021800 & -0.52786400 \\ \mathrm{C} & -0.83516300 & 1.96815200 & -0.58185900 \\ \mathrm{~N} & -1.44799100 & 0.84849400 & -0.37390300 \\ \mathrm{~N} & -2.79445400 & 0.77350600 & -0.27190600 \\ \mathrm{C} & -3.21692300 & -0.45277000 & 0.00129000 \\ \mathrm{~S} & -2.17742700 & -1.85003000 & 0.20961300 \\ \mathrm{~N} & -4.54882700 & -0.62925000 & 0.12618500 \\ \mathrm{C} & -5.42672100 & 0.51170200 & -0.10962700 \\ \mathrm{H} & -6.45259400 & 0.21004900 & 0.09150800 \\ \mathrm{H} & -5.15894300 & 1.34139700 & 0.54647000 \\ \mathrm{H} & -5.34923700 & 0.86124500 & -1.14258200 \\ \mathrm{C} & -1.50529700 & 3.29216500 & -0.70042300 \\ \mathrm{H} & -2.57543900 & 3.18968200 & -0.53717800 \\ \mathrm{H} & -1.08149500 & 3.97893400 & 0.03810900 \\ \mathrm{H} & -1.32927300 & 3.72452000 & -1.68954200 \\ \mathrm{C} & 4.97702100 & -0.19743400 & -0.64593800 \\ \mathrm{H} & 4.89255300 & 0.58459500 & 0.11167100 \\ \mathrm{H} & 5.96818800 & -0.64211000 & -0.59940900 \\ \mathrm{H} & 4.82931600 & 0.26007600 & -1.62520300 \\ \mathrm{C} & 0.64743300 & 1.87083200 & -0.62565100 \\ \mathrm{C} & 1.50574100 & 2.95371200 & -1.18688100 \\ \mathrm{H} & 1.05047500 & 3.93110400 & -1.03719800 \\ \mathrm{H} & 2.47946300 & 2.92388700 & -0.69678400 \\ \mathrm{H} & 1.65666800 & 2.78532100 & -2.25784200 \\ \mathrm{O} & 0.82014800 & 2.45892600 & 1.32814000 \\ \mathrm{O} & 2.09854500 & 2.18206500 & 1.68886700 \\ \mathrm{C} & 2.11872000 & 1.02552100 & 2.52097000 \\ \mathrm{H} & 1.58571600 & 0.20461600 & 2.03404200 \\ \mathrm{H} & 3.16930000 & 0.76716600 & 2.65607100 \\ \mathrm{H} & 1.65438700 & 1.25213800 & 3.48308600 \\ \mathrm{~N} & 3.98547400 & -1.24645500 & -0.41021800 \\ \mathrm{C} & 4.42259400 & -2.61144800 & -0.14659000 \\ \mathrm{H} & 4.21001000 & -2.90035100 & 0.88600700 \\ \mathrm{H} & 3.91499300 & -3.31283400 & -0.81129500 \\ \mathrm{H} & 5.49450200 & -2.67253100 & -0.31980500 \\ \mathrm{C} & -5.12800600 & -1.94730100 & 0.34867600 \\ \mathrm{H} & -4.99810100 & -2.59874900 & -0.52028300 \\ \mathrm{H} & -4.67283800 & -2.43186700 & 1.21378900 \\ & & & \\ & & & \\ & -1.900\end{array}$




\section{di-(methylperxoyl) adduct derived from $\mathrm{CuATSM}_{2}$ (THF)}

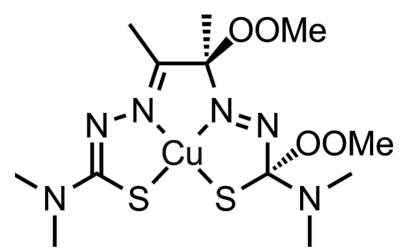

\section{MN15/CBSB7+ Enthalpy $=\quad-3536.631739$}

$\mathrm{MN15/CBSB7+}$ Free Energy= $\quad-3536.725715$

\begin{tabular}{|c|c|c|c|}
\hline & & & \\
\hline S & -1.43863800 & -1.56802300 & -1.22497400 \\
\hline $\mathrm{N}$ & -2.13498000 & 0.53339000 & 0.44995100 \\
\hline $\mathrm{N}$ & -0.97591600 & 0.82229400 & 0.18934300 \\
\hline $\mathrm{C}$ & 1.03040900 & 2.07401200 & 0.63056900 \\
\hline $\mathrm{N}$ & 1.56816800 & 0.96489900 & 0.24779700 \\
\hline $\mathrm{N}$ & 2.90565200 & 0.78624500 & 0.33462200 \\
\hline $\mathrm{C}$ & 3.30590200 & -0.38807200 & -0.14185400 \\
\hline S & 2.25718800 & -1.61403100 & -0.81896600 \\
\hline $\mathrm{N}$ & 4.62834000 & -0.64088000 & -0.09716500 \\
\hline $\mathrm{C}$ & 5.51803800 & 0.38240300 & 0.44298900 \\
\hline $\mathrm{H}$ & 5.24997700 & 0.62221100 & 1.47378400 \\
\hline $\mathrm{H}$ & 6.53887800 & 0.00764900 & 0.41414700 \\
\hline $\mathrm{H}$ & 5.45401300 & 1.30179100 & -0.14348100 \\
\hline $\mathrm{C}$ & 1.78509200 & 3.24541400 & 1.15658800 \\
\hline $\mathrm{H}$ & 1.36492900 & 4.17167100 & 0.75863100 \\
\hline $\mathrm{H}$ & 1.70983000 & 3.28646700 & 2.24812400 \\
\hline $\mathrm{H}$ & 2.83548500 & 3.16379500 & 0.88456600 \\
\hline $\mathrm{C}$ & -0.47803900 & 2.19214000 & 0.46110300 \\
\hline $\mathrm{C}$ & -1.18504700 & 2.86940800 & 1.61365600 \\
\hline $\mathrm{H}$ & -0.81107400 & 3.88820800 & 1.71704400 \\
\hline $\mathrm{H}$ & -2.25691600 & 2.89476200 & 1.42825100 \\
\hline $\mathrm{H}$ & -0.99309000 & 2.31990100 & 2.53690800 \\
\hline 0 & -0.60221400 & 2.89611000 & -0.76672200 \\
\hline 0 & -1.99033000 & 3.12377800 & -1.02527100 \\
\hline $\mathrm{C}$ & -2.26415800 & 2.51379300 & -2.27965800 \\
\hline $\mathrm{H}$ & -2.07556100 & 1.43672700 & -2.24089300 \\
\hline $\mathrm{H}$ & -3.32368100 & 2.70165100 & -2.45399000 \\
\hline $\mathrm{H}$ & -1.66872700 & 2.97567700 & -3.06944700 \\
\hline $\mathrm{Cu}$ & 0.37985300 & -0.46453600 & -0.46505300 \\
\hline $\mathrm{C}$ & 5.18135700 & -1.88862700 & -0.60805300 \\
\hline $\mathrm{H}$ & 4.75020100 & -2.75069800 & -0.09443100 \\
\hline $\mathrm{H}$ & 4.99609900 & -1.99780100 & -1.67954100 \\
\hline $\mathrm{H}$ & 6.25591300 & -1.88384400 & -0.43802800 \\
\hline $\mathrm{C}$ & -2.44415100 & -0.90398900 & 0.11876700 \\
\hline 0 & -2.19320300 & -1.56554300 & 1.37817900 \\
\hline 0 & -0.79959100 & -1.52278400 & 1.68259000 \\
\hline $\mathrm{C}$ & -0.36084700 & -2.87218900 & 1.79019800 \\
\hline $\mathrm{H}$ & -0.49688400 & -3.38845700 & 0.83794300 \\
\hline $\mathrm{H}$ & 0.70053800 & -2.80189900 & 2.03095500 \\
\hline $\mathrm{H}$ & -0.89794300 & -3.38057900 & 2.59299000 \\
\hline $\mathrm{N}$ & -3.84267900 & -0.96848900 & -0.11618300 \\
\hline $\mathrm{C}$ & -4.34123200 & -2.20370500 & -0.71444400 \\
\hline $\mathrm{H}$ & -4.17955900 & -3.07396400 & -0.06350700 \\
\hline $\mathrm{H}$ & -5.41466400 & -2.08719000 & -0.87330700 \\
\hline $\mathrm{H}$ & -3.86758400 & -2.38655200 & -1.67730800 \\
\hline $\mathrm{C}$ & -4.68314600 & -0.55798600 & 1.01390900 \\
\hline $\mathrm{H}$ & -4.26732900 & 0.32464000 & 1.49543600 \\
\hline
\end{tabular}




\section{CuATSP (THF)}<smiles>CC1=C(C)N2N=C(Nc3ccccc3)SC23SC(Nc2ccccc2)=NN13</smiles>

\section{MN15/CBSB7+ Enthalpy $=\quad-3461.049024$ \\ MN15/CBSB7+ Free Energy $=\quad-3461.134524$}

\begin{tabular}{|c|c|c|c|}
\hline $\mathrm{Cu}$ & -0.00620500 & -0.86128800 & 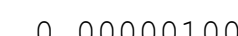 \\
\hline $\mathrm{S}$ & -1.85902500 & -2.12770000 & -0.00002300 \\
\hline $\mathrm{C}$ & -2.96411400 & -0.76660800 & 0.00001000 \\
\hline $\mathrm{N}$ & -2.62238900 & 0.50100700 & 0.00004600 \\
\hline $\mathrm{N}$ & -1.27736300 & 0.66607600 & 0.00004700 \\
\hline $\mathrm{C}$ & -0.73100500 & 1.83929700 & 0.00007300 \\
\hline $\mathrm{C}$ & 0.75496100 & 1.82197000 & 0.00006500 \\
\hline $\mathrm{N}$ & 1.27622300 & 0.63805100 & 0.00003100 \\
\hline $\mathrm{N}$ & 2.61802100 & 0.47605100 & 0.00001700 \\
\hline $\mathrm{C}$ & 2.95883500 & -0.79427100 & -0.00002100 \\
\hline $\mathrm{S}$ & 1.84632700 & -2.14682700 & -0.00004300 \\
\hline $\mathrm{N}$ & 4.27235200 & -1.14421000 & -0.00004600 \\
\hline $\mathrm{H}$ & 4.42728100 & -2.14652500 & -0.00007800 \\
\hline C & 1.59449300 & 3.05398700 & 0.00009400 \\
\hline $\mathrm{H}$ & 0.99478400 & 3.96158200 & 0.00012800 \\
\hline $\mathrm{H}$ & 2.24748300 & 3.05264100 & 0.87727700 \\
\hline $\mathrm{H}$ & 2.24746400 & 3.05269400 & -0.87710300 \\
\hline $\mathrm{C}$ & -1.49167500 & 3.12075800 & 0.00010600 \\
\hline $\mathrm{H}$ & -1.23371600 & 3.71470900 & -0.88084700 \\
\hline $\mathrm{H}$ & -2.56120800 & 2.92482600 & 0.00009100 \\
\hline $\mathrm{H}$ & -1.23373100 & 3.71465400 & 0.88110100 \\
\hline $\mathrm{N}$ & -4.27814000 & -1.12211500 & -0.00000700 \\
\hline $\mathrm{H}$ & -4.42603600 & -2.12539900 & -0.00005300 \\
\hline C & 5.44840300 & -0.36579300 & -0.00004800 \\
\hline C & 6.65515500 & -1.08137900 & -0.00011500 \\
\hline C & 5.48364900 & 1.03305600 & 0.00001400 \\
\hline $\mathrm{C}$ & 7.87273600 & -0.41680500 & -0.00012000 \\
\hline $\mathrm{H}$ & 6.62975200 & -2.16668000 & -0.00016600 \\
\hline $\mathrm{C}$ & 6.71591500 & 1.68649900 & 0.00000900 \\
\hline $\mathrm{H}$ & 4.56412600 & 1.59827200 & 0.00006600 \\
\hline C & 7.91245900 & 0.97760400 & -0.00005800 \\
\hline $\mathrm{H}$ & 8.79198900 & -0.99086500 & -0.00017300 \\
\hline $\mathrm{H}$ & 6.73026700 & 2.77060700 & 0.00005800 \\
\hline $\mathrm{H}$ & 8.86155000 & 1.49993600 & -0.00006100 \\
\hline $\mathrm{C}$ & -5.46033300 & -0.35458400 & -0.00000100 \\
\hline C & -5.50773800 & 1.04332600 & 0.00013900 \\
\hline C & -6.66156800 & -1.07955500 & -0.00013600 \\
\hline C & -6.74440900 & 1.68799700 & 0.00014100 \\
\hline $\mathrm{H}$ & -4.59257600 & 1.61443900 & 0.00024700 \\
\hline $\mathrm{C}$ & -7.88428100 & -0.42438300 & -0.00013200 \\
\hline $\mathrm{H}$ & -6.62760700 & -2.16464500 & -0.00024700 \\
\hline C & -7.93535400 & 0.96969600 & 0.00000700 \\
\hline $\mathrm{H}$ & -6.76687000 & 2.77195200 & 0.00025100 \\
\hline $\mathrm{H}$ & -8.79896600 & -1.00573100 & -0.00023800 \\
\hline $\mathrm{H}$ & -8.88858400 & 1.48440500 & 0.00001000 \\
\hline
\end{tabular}

\section{Aminyl radical derived from CuATSP (THF)}


<smiles>CC1=C(C)N2N=C(Nc3ccccc3)SC23SC(Nc2ccccc2)=NN13</smiles>

\section{MN15/CBSB7+ Enthalpy $=\quad-3460.405102$ MN15/CBSB7+ Free Energy= $\quad-3460.491798$}

\begin{tabular}{|c|c|c|c|}
\hline 3 & & & \\
\hline $\mathrm{Cu}$ & 0.05712700 & -1.02193700 & -0.0202200 \\
\hline $\mathrm{S}$ & 1.84829700 & -2.33941200 & -0.03476300 \\
\hline $\mathrm{C}$ & 3.04416300 & -1.04683800 & -0.06584600 \\
\hline $\mathrm{N}$ & 2.64793100 & 0.30162400 & -0.13099200 \\
\hline $\mathrm{N}$ & 1.37902700 & 0.45820600 & -0.11166100 \\
\hline $\mathrm{C}$ & 0.85007600 & 1.70388000 & -0.2028030 \\
\hline $\mathrm{C}$ & -0.57308200 & 1.73529100 & -0.1670070 \\
\hline $\mathrm{N}$ & -1.15349400 & 0.53674200 & -0.0820870 \\
\hline $\mathrm{N}$ & -2.46810700 & 0.44723300 & -0.0389990 \\
\hline C & -2.89142600 & -0.82195000 & 0.04337800 \\
\hline $\mathrm{S}$ & -1.86179700 & -2.21247100 & 0.0986590 \\
\hline $\mathrm{N}$ & -4.21899700 & -1.05760300 & 0.0897360 \\
\hline $\mathrm{H}$ & -4.45108000 & -2.04385800 & 0.1602290 \\
\hline $\mathrm{C}$ & -1.41705900 & 2.96291700 & -0.2183570 \\
\hline $\mathrm{H}$ & -0.82777200 & 3.87474200 & -0.25509300 \\
\hline $\mathrm{H}$ & -2.07032800 & 2.92131300 & -1.0945090 \\
\hline $\mathrm{H}$ & -2.06947500 & 2.99161300 & 0.6585780 \\
\hline $\mathrm{C}$ & 1.75691400 & 2.87580000 & -0.3209400 \\
\hline $\mathrm{H}$ & 1.21489100 & 3.81437200 & -0.3927030 \\
\hline $\mathrm{H}$ & 2.42879700 & 2.91146000 & 0.5415240 \\
\hline $\mathrm{H}$ & 2.39254400 & 2.75938900 & -1.2031490 \\
\hline $\mathrm{N}$ & 4.29556700 & -1.34817000 & -0.0805430 \\
\hline C & 5.30947500 & -0.38982300 & 0.0449160 \\
\hline C & 6.42783700 & -0.50544600 & -0.7919750 \\
\hline C & 5.29702000 & 0.60661200 & 1.0332360 \\
\hline C & 7.48129900 & 0.39501600 & -0.6859740 \\
\hline $\mathrm{H}$ & 6.44452700 & -1.30098700 & -1.5282640 \\
\hline C & 6.36571400 & 1.48866800 & 1.1491560 \\
\hline $\mathrm{H}$ & 4.45869800 & 0.66446600 & 1.7175290 \\
\hline C & 7.45611200 & 1.39662100 & 0.2847990 \\
\hline $\mathrm{H}$ & 8.33085900 & 0.30684800 & -1.3535540 \\
\hline $\mathrm{H}$ & 6.34891200 & 2.24881900 & 1.9220990 \\
\hline $\mathrm{H}$ & 8.28534400 & 2.08812100 & 0.3773430 \\
\hline C & -5.34419600 & -0.20061400 & 0.06432400 \\
\hline C & -5.29546600 & 1.19159800 & -0.0610960 \\
\hline $\mathrm{C}$ & -6.58680300 & -0.84081600 & 0.1706680 \\
\hline C & -6.48644600 & 1.91550900 & -0.0767400 \\
\hline $\mathrm{H}$ & -4.34730900 & 1.69989600 & -0.1449530 \\
\hline C & -7.76347100 & -0.10625100 & 0.15340200 \\
\hline $\mathrm{H}$ & -6.62336100 & -1.92130200 & 0.26718300 \\
\hline C & -7.72061500 & 1.28182200 & 0.0294420 \\
\hline $\mathrm{H}$ & -6.43925600 & 2.99396800 & -0.1745320 \\
\hline $\mathrm{H}$ & -8.71360500 & -0.62028500 & 0.2370580 \\
\hline $\mathrm{H}$ & -8.63683200 & 1.85952800 & 0.015 \\
\hline
\end{tabular}

\section{Peroxyl adduct derived from CuATSP (THF)}




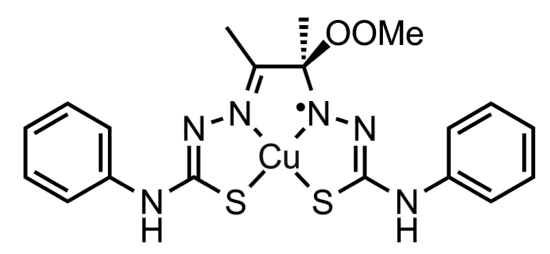

\section{MN15/CBSB7+ Enthalpy $=\quad-3651.097734$ MN15/CBSB7+ Free Energy= $\quad-3651.192554$}

\begin{tabular}{|c|c|c|c|}
\hline \multicolumn{4}{|l|}{03} \\
\hline $\mathrm{Cu}$ & -0.23699700 & -1.14452300 & 0.06112500 \\
\hline $\mathrm{S}$ & 1.57465300 & -2.51243900 & 0.16619200 \\
\hline C & 2.71452500 & -1.23451200 & -0.06375100 \\
\hline $\mathrm{N}$ & 2.37022000 & 0.04974600 & -0.23155300 \\
\hline $\mathrm{N}$ & 1.08523300 & 0.25233100 & -0.15141000 \\
\hline C & -0.89744400 & 1.58367100 & -0.25826800 \\
\hline $\mathrm{N}$ & -1.43268600 & 0.42132900 & -0.12474000 \\
\hline $\mathrm{N}$ & -2.78849200 & 0.30610500 & -0.10940600 \\
\hline $\mathrm{C}$ & -3.18192500 & -0.93494200 & 0.04955200 \\
\hline $\mathrm{S}$ & -2.13487400 & -2.32647700 & 0.22189300 \\
\hline $\mathrm{N}$ & -4.51217800 & -1.22565200 & 0.09246000 \\
\hline $\mathrm{H}$ & -4.71030400 & -2.21105100 & 0.22534100 \\
\hline C & -1.68636500 & 2.84065900 & -0.37991200 \\
\hline $\mathrm{H}$ & -2.17796600 & 2.87778600 & -1.35727600 \\
\hline $\mathrm{H}$ & -2.47443200 & 2.85461000 & 0.37589900 \\
\hline $\mathrm{H}$ & -1.04743400 & 3.71457200 & -0.26372400 \\
\hline C & 0.62435100 & 1.62383200 & -0.29919300 \\
\hline C & 1.14126700 & 2.25995200 & -1.58151400 \\
\hline $\mathrm{H}$ & 2.22983400 & 2.22301800 & -1.58829800 \\
\hline $\mathrm{H}$ & 0.75725600 & 1.70435400 & -2.43871800 \\
\hline $\mathrm{H}$ & 0.81213700 & 3.29812700 & -1.64404800 \\
\hline 0 & 0.93893700 & 2.40323400 & 0.85455800 \\
\hline 0 & 2.31125400 & 2.80086500 & 0.81454900 \\
\hline C & 2.89137000 & 2.34040000 & 2.02367000 \\
\hline $\mathrm{H}$ & 2.87040200 & 1.24890700 & 2.07264300 \\
\hline $\mathrm{H}$ & 3.92404300 & 2.69061000 & 1.99038100 \\
\hline $\mathrm{H}$ & 2.38091900 & 2.77415500 & 2.88617000 \\
\hline $\mathrm{N}$ & 4.02850400 & -1.55991000 & -0.07820100 \\
\hline $\mathrm{H}$ & 4.20325400 & -2.55499000 & 0.02187200 \\
\hline C & 5.19499900 & -0.76903000 & -0.18133000 \\
\hline C & 5.21692200 & 0.62942400 & -0.24428800 \\
\hline $\mathrm{C}$ & 6.40467500 & -1.47768800 & -0.20357100 \\
\hline $\mathrm{C}$ & 6.44245200 & 1.28826700 & -0.32726000 \\
\hline $\mathrm{H}$ & 4.29659800 & 1.19434800 & -0.23100200 \\
\hline $\mathrm{C}$ & 7.61619200 & -0.80667100 & -0.28742700 \\
\hline $\mathrm{H}$ & 6.38758200 & -2.56191500 & -0.15396000 \\
\hline C & 7.64356400 & 0.58597300 & -0.34989000 \\
\hline $\mathrm{H}$ & 6.44831800 & 2.37132700 & -0.37508200 \\
\hline $\mathrm{H}$ & 8.53898800 & -1.37443100 & -0.30348900 \\
\hline $\mathrm{H}$ & 8.58731200 & 1.11374000 & -0.41516400 \\
\hline C & -5.65217600 & -0.40168000 & 0.00155200 \\
\hline C & -6.88851400 & -1.05054600 & 0.14037800 \\
\hline $\mathrm{C}$ & -5.62843000 & 0.98002000 & -0.21848000 \\
\hline $\mathrm{C}$ & -8.07609400 & -0.33816500 & 0.06277200 \\
\hline $\mathrm{H}$ & -6.90930100 & -2.12249900 & 0.31086000 \\
\hline C & -6.83125300 & 1.68260800 & -0.29337300 \\
\hline $\mathrm{H}$ & -4.68604900 & 1.49436000 & -0.32937300 \\
\hline $\mathrm{C}$ & -8.05659000 & 1.03969900 & -0.15496000 \\
\hline $\mathrm{H}$ & -9.01862100 & -0.86172400 & 0.17350200 \\
\hline $\mathrm{H}$ & -6.79893500 & 2.75284600 & -0.46410300 \\
\hline $\mathrm{H}$ & -8.98205500 & 1.59959300 & -0.21557000 \\
\hline
\end{tabular}


TS structure for addition of $\mathrm{MeOO} \bullet$ to CuATSP (THF)<smiles>CC1=C(C)C23NC(Nc4ccccc4)=NN(C1C)N2N=C(Nc1ccccc1)S3</smiles>

$\begin{array}{ll}\text { MN15/CBSB7+ Enthalpy }= & -3651.069067 \\ \text { MN15/CBSB7+ Free Energy= } & -3651.163739 \\ \text { Imaginary frequency: }-548.69 & \end{array}$

03

$\mathrm{Cu}$

$\mathrm{S}$

C

$\mathrm{N}$

$\mathrm{N}$

C

$\mathrm{N}$

$\mathrm{N}$

C

S

$\mathrm{N}$

$\mathrm{H}$

C

$\mathrm{H}$

$\mathrm{H}$

$\mathrm{H}$

C

C

$\mathrm{H}$

$\mathrm{H}$

$\mathrm{H}$

O

O

C

$\mathrm{H}$

$\mathrm{H}$

$\mathrm{H}$

N

$\mathrm{H}$

C

C

C

C

$\mathrm{H}$

C

$\mathrm{H}$

C

$\mathrm{H}$

$\mathrm{H}$

$\mathrm{H}$

C

C

C

C

$\mathrm{H}$

C

$\mathrm{H}$

C

\begin{abstract}
$-0.19299600$
1. 63446900

2.76798400

2.43969000

1.13172400

$-0.83927100$

$-1.41081700$

$-2.76213300$

$-3.14006700$

$-2.07411000$

$-4.46370500$

$-4.64034700$

$-1.54841600$

$-1.37310400$

$-2.61719300$

$-1.14983400$

0.65096600

1. 47941900

2.46079100

1. 61555200

1. 00528400

0.78358200

2. 05934400

2.09079700

1.56414700

3.14446300

1. 62522800

4.08024800

4. 26991500

5.22053100

5. 22448100

6.40250000

6.40774800

4. 31815100

7.57760700

6.39222100

7.58497400

6.40583800

8.48568600

8.49885200

$-5.62101300$

$-6.83889500$

$-5.62980900$

$-8.04019800$

$-6.83465900$

$-6.84527700$

$-4.70174300$

$-8.05261500$
\end{abstract}

$-1.06370000$

$-2.39704300$

$-1.12513300$

0.13658100

0.34042000

1. 62628500

0.48205200

0.36497500

$-0.86787700$

$-2.24298800$

$-1.17453400$

$-2.15069600$

2. 92828200

3. 36886500

2. 79763300

3. 62249300

1. 58015000

2.68649400

2. 67283100

2.53214300

3. 65188700

2. 15586000

1. 90517000

0.76299800

$-0.07046000$

0.51805400

1.00455500

$-1.44940200$

$-2.44099500$

$-0.61369900$

0.72999700

$-1.21297300$

1.45900100

1.19768000

$-0.47516900$

$-2.25813000$

0.86846100

2.50005800

$-0.95146600$

1. 44715700

$-0.37828200$

$-1.02610600$

0.97462900

$-0.33878100$

$-2.07658700$

1. 65280800

1.48560700

1. 01152100
$-0.07695000$

0.11480100

$-0.20657300$

$-0.48584100$

$-0.41281600$

$-0.49969000$

$-0.32400500$

$-0.25260800$

$-0.01779100$

0.20503800

0.07984600

0.28970300

$-0.61635600$

$-1.60188400$

$-0.46414900$

0.12898800

$-0.51369200$

$-1.07594400$

$-0.60039600$

$-2.15061500$

$-0.90826100$

1. 40681200

1. 78468800

2.64016900

2.16780100

2.77816500

3.59729300

$-0.16118800$

$-0.05996900$

$-0.25554400$

0.13165000

$-0.70208800$

0.04748800

0.49272700

$-0.77594400$

$-0.99362300$

$-0.40645500$

0.34914100

$-1.12622000$

$-0.46674300$

$-0.03409700$

0.22157600

$-0.38925600$

0.12857000

0.49542800

$-0.47753500$

$-0.59322400$

$-0.22174900$ 


$\begin{array}{rrrr}\mathrm{H} & -8.96840600 & -0.86029900 & 0.33135400 \\ \mathrm{H} & -6.83795500 & 2.70130000 & -0.75339500 \\ \mathrm{H} & -8.98889300 & 1.55165900 & -0.29415000\end{array}$

$N$-methylimine derived from peroxyl-substituted CuATSP (THF)<smiles></smiles>

\section{MN15/CBSB7+ Enthalpy $=\quad-3650.482044$ \\ $\mathrm{MN} 15 / \mathrm{CBSB} 7+$ Free Energy $=\quad-3650.576992$}

$\begin{array}{lrrr}\text { O 2 } & & & \\ \mathrm{Cu} & -0.14330500 & -1.14622500 & 0.24480600 \\ \mathrm{~S} & 1.60968500 & -2.54928700 & 0.33251200 \\ \mathrm{C} & 2.82492700 & -1.35211700 & -0.03427600 \\ \mathrm{~N} & 2.45828600 & 0.05029000 & -0.04715100 \\ \mathrm{~N} & 1.25232900 & 0.24008300 & 0.11219000 \\ \mathrm{C} & -0.72610900 & 1.62533700 & 0.05688900 \\ \mathrm{~N} & -1.29022500 & 0.46735700 & 0.05775400 \\ \mathrm{~N} & -2.63678600 & 0.36561500 & -0.09206400 \\ \mathrm{C} & -3.05857000 & -0.87601400 & -0.02265100 \\ \mathrm{~S} & -2.05595800 & -2.28865000 & 0.22286100 \\ \mathrm{~N} & -4.38556800 & -1.15069500 & -0.14103600 \\ \mathrm{H} & -4.60853600 & -2.13564700 & -0.04877400 \\ \mathrm{C} & -1.43886600 & 2.92472300 & -0.07557300 \\ \mathrm{H} & -1.00680900 & 3.65802800 & 0.60843500 \\ \mathrm{H} & -1.32631800 & 3.31079000 & -1.09369400 \\ \mathrm{H} & -2.49891700 & 2.79750900 & 0.13458400 \\ \mathrm{~N} & 4.05088900 & -1.65450200 & -0.22763500 \\ \mathrm{C} & 0.78007300 & 1.63639800 & 0.28571500 \\ \mathrm{C} & 1.54128700 & 2.62102900 & -0.57417600 \\ \mathrm{H} & 1.20177800 & 3.63297700 & -0.35264700 \\ \mathrm{H} & 2.60739900 & 2.54264500 & -0.36787800 \\ \mathrm{H} & 1.36248700 & 2.40381200 & -1.62880100 \\ \mathrm{O} & 0.86186000 & 1.91384700 & 1.67535500 \\ \mathrm{O} & 2.23549900 & 2.04464800 & 2.04487200 \\ \mathrm{C} & 2.44753200 & 1.11134600 & 3.09528000 \\ \mathrm{H} & 2.25120200 & 0.08971300 & 2.75688000 \\ \mathrm{H} & 3.49916300 & 1.22286100 & 3.35826100 \\ \mathrm{H} & 1.81917800 & 1.35021600 & 3.95501000 \\ \mathrm{C} & 5.01284000 & -0.72737900 & -0.65567400 \\ \mathrm{C} & 4.78993900 & 0.15331400 & -1.72376600 \\ \mathrm{C} & 6.27086500 & -0.75882500 & -0.04099800 \\ \mathrm{C} & 5.79851400 & 1.01912100 & -2.13347300 \\ \mathrm{H} & 3.83892500 & 0.13188300 & -2.24396100 \\ \mathrm{C} & 7.26530700 & 0.12523600 & -0.44114900 \\ \mathrm{H} & 6.44244400 & -1.47076300 & 0.75808600 \\ \mathrm{C} & 7.03384300 & 1.01807500 & -1.48823200 \\ \mathrm{H} & 5.61995400 & 1.69181700 & -2.96448100 \\ \mathrm{H} & 8.22845700 & 0.10882000 & 0.05566100 \\ \mathrm{H} & -7.81643000 & 1.69512700 & -1.80965100 \\ \mathrm{C} & -5.50331900 & -0.31184300 & -0.33444800 \\ \mathrm{C} & -5.43906700 & 1.06020700 & -0.59739500 \\ \mathrm{C} & -6.75628600 & -0.93888500 & -0.27023300 \\ \mathrm{C} & -6.62000000 & 1.77815200 & -0.78497500 \\ \mathrm{H} & -4.48250000 & 1.55567100 & -0.65666700 \\ \mathrm{C} & -6242300 & -0.21208700 & -0.46086900 \\ \mathrm{H} & -80761800 & -2.00426200 & -0.06810500 \\ \mathrm{C} & & & \\ & & & \end{array}$




$\begin{array}{lrrr}\mathrm{H} & -6.55677700 & 2.84123400 & -0.98813500 \\ \mathrm{H} & -8.87942100 & -0.71779800 & -0.40615400 \\ \mathrm{H} & -8.77148900 & 1.72846800 & -0.86672600\end{array}$

Truncated analogue of CuATSM<smiles>CNC1=NN2C=CN3N=CS[C@@]32S1</smiles>

CBS-QB3 Enthalpy $=-2902.655030$

CBS-QB3 Free Energy $=-2902.711845$

B3LYP/CBSB7 Enthalpy = -2905.391379

B3LYP/CBSB7 Free Energy = -2905.447990

02
S
C
$\mathrm{N}$
$\mathrm{N}$
$\mathrm{C}$
$\mathrm{N}$
$\mathrm{N}$
$\mathrm{C}$
$\mathrm{S}$
$\mathrm{C}$
$\mathrm{H}$
$\mathrm{H}$
$\mathrm{H}$
$\mathrm{C}$
$\mathrm{N}$
$\mathrm{H}$
$\mathrm{Cu}$
$\mathrm{H}$
$\mathrm{H}$
$\mathrm{H}$

-1.24913400
-2.36767400
-2.04473900
-0.72932500
1.27517000
1.85071900
3.20528100
3.54686200
2.51812300
-4.73833300
-4.67294200
-5.69619200
-4.67296900
-0.17692000
-3.67792400
-3.92354800
0.60320900
4.61671500
1.85690500
-0.76758300

$-1.65578700$

$-0.00000100$

$-0.29886000$

1.00339600

0.00000000

1.18142600

2.36265400

1.20407300

1.06723600

$-0.19294100$

$-1.58904900$

0.41610000

1.05291400

$-0.10316900$

1.05289400

2.36033400

$-0.58449000$

$-1.56122400$

$-0.33373700$

$-0.38267500$

3.28097300

3.27083400

0.00000100

0.00000000

$-0.00000100$

0.00000100

0.00000000

$-0.00000100$

$-0.00000100$

$-0.00000200$

$-0.88460500$

$-0.00002200$

0.88461900

0.00000000

0.00000200

0.00000100

0.00000100

$-0.00000100$

$-0.00000300$

$-0.00000100$

\section{Truncated analogue of CuATSM}<smiles>CNC1=NN2C=CN3N=CS[C@@]32S1</smiles>

\section{wB97X/CBSB7 Enthalpy= wB97X/CBSB7 Free Energy=}

$0^{2} 2$
$S$
$C$
$N$
$N$
$C$
$N$
$N$
$C$
$S$
$C$

$-2905.204494$ $-2905.260016$

$$
\begin{array}{r}
-1.63321800 \\
-0.29040100 \\
1.00600900 \\
1.18178600 \\
2.34755600 \\
1.19302600 \\
1.04910800 \\
-0.20707700 \\
-1.57931500 \\
0.41137000
\end{array}
$$


1. 04785500

$-0.10054300$

1.04784300

2.35174200

$-0.58992300$

$-1.56627300$

$-0.33419700$

$-0.41441300$

3.25837600

3. 26022000
-0.88477700
-0.00000400
0.88479200
0.00000000
-0.00000100
0.00000200
0.00000000
0.00000600
0.00000300
-0.00000100

\section{Truncated analogue of CuATSM}<smiles>CNC1=NN2C=CN3N=CS[C@@]32S1</smiles>

\section{M06-2X/Def2TZVP Enthalpy $=\quad-2905.200290$ M06-2X/Def2TZVP Free Energy $=\quad-2905.255920$}

\footnotetext{
02

$\mathrm{S}$

C

$\mathrm{N}$

$\mathrm{N}$

C

$\mathrm{N}$

$\mathrm{N}$

C

S

C

$\mathrm{H}$

$\mathrm{H}$

$\mathrm{H}$

C

$\mathrm{N}$

$\mathrm{H}$

$\mathrm{Cu}$

$\mathrm{H}$

$\mathrm{H}$

$\mathrm{H}$
}

$$
\begin{array}{r}
1.31468800 \\
2.39046900 \\
2.06325400 \\
0.74945400 \\
-1.25276400 \\
-1.84096900 \\
-3.19792900 \\
-3.57244800 \\
-2.61937500 \\
4.72246500 \\
4.63139800 \\
5.69463500 \\
4.63124800 \\
0.21159500 \\
3.69980400 \\
3.96585700 \\
-0.61031900 \\
-4.64909500 \\
-1.81846200 \\
0.80746300
\end{array}
$$

$$
\begin{array}{r}
0.00007800 \\
0.00004600 \\
0.00005400 \\
0.00004000 \\
-0.00001900 \\
-0.00003700 \\
-0.00008900 \\
-0.00007800 \\
-0.00002000 \\
-0.00007300 \\
0.88279400 \\
-0.00016400 \\
-0.88290200 \\
0.00002400 \\
-0.00000700 \\
-0.00002600 \\
0.00001300 \\
-0.00012900 \\
-0.00004300 \\
0.00003100
\end{array}
$$

\section{Truncated analogue of CuATSM}<smiles>CNC1=NN2C=CN3N=CS[C@@]32S1</smiles>

$-2905.091134$

\section{$-2905.147083$}

$$
\begin{array}{r}
-1.67927900 \\
-0.30388700 \\
0.99147500 \\
1.16903000 \\
2.36171100 \\
1.22194300 \\
1.12129900
\end{array}
$$

0.00002600

0.00004600

0.00003200

0.00001600

$-0.00001400$

$-0.00002000$

$-0.00003400$ 
$-0.12321000$

$-1.55976500$

0.46213500

1.09345200

$-0.02516700$

1.09348400

2.34208600

$-0.56911300$

$-1.54017900$

$-0.38072000$

$-0.26762600$

3.28866300

3. 24750900
$-0.00000600$

$-0.00000900$

$-0.00003200$

0.88468200

$-0.00008300$

$-0.88471500$

0.00000600

$-0.00000600$

$-0.00002900$

0.00000200

$-0.00009200$

$-0.00002300$

0.00000900

Truncated analogue of CuATSM<smiles>CNC1=NN2C=CN3N=CSC32S1</smiles>

\section{M06-2X-D3/CBSB7 Enthalpy= \\ $-2905.054490$ \\ M06-2X-D3/CBSB7 Free Energy= $-2905.109889$}

$\mathrm{O}^{2}$
$\mathrm{~S}$
$\mathrm{C}$
$\mathrm{N}$
$\mathrm{N}$
$\mathrm{C}$
$\mathrm{N}$
$\mathrm{N}$
$\mathrm{C}$
$\mathrm{S}$
$\mathrm{C}$
$\mathrm{H}$
$\mathrm{H}$
$\mathrm{H}$
$\mathrm{C}$
$\mathrm{N}$
$\mathrm{H}$
$\mathrm{Cu}$
$\mathrm{H}$
$\mathrm{H}$
$\mathrm{H}$

$$
\begin{array}{r}
1.28557500 \\
2.37803000 \\
2.05500200 \\
0.73816900 \\
-1.26268900 \\
-1.83968800 \\
-3.19908500 \\
-3.55715800 \\
-2.57123500 \\
4.71370100 \\
4.62187500 \\
5.68580900 \\
4.62186300 \\
0.20294100 \\
3.68646900 \\
3.94837000 \\
-0.60609200 \\
-4.63099700 \\
-1.83893700 \\
0.80421600
\end{array}
$$$$
-1.67341800
$$$$
-0.30449300
$$$$
0.99208900
$$$$
1.16937800
$$$$
2.36018600
$$$$
\text { 1. } 21528300
$$$$
\text { 1. } 10006300
$$

$-0.14960400$

$-1.57133500$

0.45176400

1.08404500

$-0.03689200$

1.08406400

2.34414700

$-0.57648400$

$-1.54860000$

$-0.35848600$

$-0.31343400$

3. 28178300

3. 24687800

$$
\begin{array}{r}
-0.00000400 \\
0.00000300 \\
0.00000500 \\
0.00000300 \\
0.00000100 \\
-0.00000300 \\
-0.00000400 \\
0.00000000 \\
0.00000200 \\
0.00000400 \\
0.88418200 \\
-0.00000700 \\
-0.88415900 \\
0.00000300 \\
0.00000000 \\
-0.00000200 \\
-0.00000100 \\
-0.00000700 \\
0.00000300 \\
0.00000500
\end{array}
$$

\section{Truncated analogue of CuATSM}<smiles>CNC1=NN2C=CN3N=CS[C@@]32S1</smiles>

\section{M06-2X/CBSB7 Enthalpy= M06-2X/CBSB7 Free Energy=}

$-2905.053666$ $-2905.109070$

$$
\begin{gathered}
02 \\
\text { S } \\
\text { C } \\
\text { N }
\end{gathered}
$$

$$
\begin{array}{r}
-1.67320900 \\
-0.30437300 \\
0.99220300
\end{array}
$$

-0.00000400
0.00000300
0.00000500 


$\begin{array}{lrrr}\mathrm{N} & 0.73816700 & 1.16939700 & 0.00000300 \\ \mathrm{C} & -1.26280600 & 2.36006100 & 0.00000100 \\ \mathrm{~N} & -1.83976600 & 1.21516400 & -0.00000300 \\ \mathrm{~N} & -3.19914900 & 1.09991000 & -0.00000400 \\ \mathrm{C} & -3.55711900 & -0.14978000 & 0.00000000 \\ \mathrm{~S} & -2.57107500 & -1.57140100 & 0.00000200 \\ \mathrm{C} & 4.71387700 & 0.45152100 & 0.00000400 \\ \mathrm{H} & 4.62222900 & 1.08379500 & 0.88419700 \\ \mathrm{H} & 5.68586900 & -0.03736700 & -0.00000800 \\ \mathrm{H} & 4.62221700 & 1.08381300 & -0.88417400 \\ \mathrm{C} & 0.20281800 & 2.34409800 & 0.00000300 \\ \mathrm{~N} & 3.68645600 & -0.57650900 & 0.00000000 \\ \mathrm{H} & 3.94825900 & -1.54865100 & -0.00000200 \\ \mathrm{Cu} & -0.60613600 & -0.35837700 & -0.00000100 \\ \mathrm{H} & -4.63093000 & -0.31371400 & -0.00000700 \\ \mathrm{H} & -1.83912100 & 3.28160100 & 0.00000200 \\ \mathrm{H} & 0.80396100 & 3.24690000 & 0.00000500\end{array}$

Truncated analogue of CuATSM<smiles>CNC1=NN2C=CN3N=CS[C@@]32S1</smiles>

\section{M06/CBSB7 Enthalpy $=\quad-2904.897932$ M06/CBSB7 Free Energy= $\quad-2904.954869$}

02
S
C
N
N
$C$
$\mathrm{~N}$
$\mathrm{~N}$
$\mathrm{C}$
$\mathrm{S}$
$\mathrm{C}$
$\mathrm{H}$
$\mathrm{H}$
$\mathrm{H}$
$\mathrm{C}$
$\mathrm{N}$
$\mathrm{H}$
$\mathrm{Cu}$
$\mathrm{H}$
$\mathrm{H}$
$\mathrm{H}$

$$
\begin{array}{r}
1.22446600 \\
2.34017400 \\
2.03107900 \\
0.72096500 \\
-1.27911800 \\
-1.84272600 \\
-3.19005300 \\
-3.50854000 \\
-2.46847600 \\
4.69420600 \\
4.62664900 \\
5.65800900 \\
4.62662900 \\
0.17208900 \\
3.64503600 \\
3.88603800 \\
-0.59563700 \\
-4.57815400 \\
-1.87062800 \\
0.76613000
\end{array}
$$$$
-1.64330700
$$$$
-0.29592400
$$$$
1.00452200
$$$$
1.18464900
$$$$
2.35475100
$$$$
1.19792500
$$$$
1.04880700
$$$$
-0.21341700
$$$$
-1.59122800
$$$$
0.40673600
$$$$
1.04652600
$$$$
-0.10316200
$$$$
1.04655300
$$$$
\text { 2. } 35718600
$$$$
-0.59112300
$$$$
-1.57026900
$$$$
-0.32252600
$$$$
-0.42182200
$$$$
3.27000500
$$

3.26853200

$$
\begin{array}{r}
-0.00000300 \\
0.00000300 \\
0.00000400 \\
0.00000300 \\
0.00000000 \\
-0.00000400 \\
-0.00000500 \\
-0.00000100 \\
0.00000200 \\
0.00000400 \\
0.88459300 \\
-0.00001400 \\
-0.88456300 \\
0.00000200 \\
0.00000000 \\
-0.00000100 \\
-0.00000100 \\
-0.00000800 \\
0.00000200 \\
0.00000300
\end{array}
$$

\section{Truncated analogue of CuATSM}<smiles>CNC1=NN2C=CN3N=CS[C@@]32S1</smiles>

$\begin{array}{ll}\text { M06/CBSB7+ Enthalpy }= & -2904.943453 \\ \text { M06/CBSB7+ Free Energy }= & -2905.000473\end{array}$ 
02

$\begin{array}{lr}\text { S } & 1.25596500 \\ \mathrm{C} & 2.35918300 \\ \mathrm{~N} & 2.04442900 \\ \mathrm{~N} & 0.73445000 \\ \mathrm{C} & -1.26726500 \\ \mathrm{~N} & -1.84408100 \\ \mathrm{~N} & -3.19338100 \\ \mathrm{C} & -3.53597100 \\ \mathrm{~S} & -2.52701700 \\ \mathrm{C} & 4.71385600 \\ \mathrm{H} & 4.64471100 \\ \mathrm{H} & 5.67894700 \\ \mathrm{H} & 4.64472900 \\ \mathrm{C} & 0.18426000 \\ \mathrm{~N} & 3.66784900 \\ \mathrm{H} & 3.91461200 \\ \mathrm{Cu} & -0.60176000 \\ \mathrm{H} & -4.61025100 \\ \mathrm{H} & -1.84964000 \\ \mathrm{H} & 0.77552000\end{array}$

$$
\begin{array}{r}
-1.65132500 \\
-0.29556300 \\
1.00350600 \\
1.18414200 \\
2.35697300 \\
1.20722300 \\
1.07676900 \\
-0.17889100 \\
-1.57769500 \\
0.41841700 \\
1.05709100 \\
-0.08931000 \\
1.05711800 \\
2.35445600 \\
-0.58331100 \\
-1.56141700 \\
-0.34938200 \\
-0.36438700 \\
3.27853300 \\
3.26809900
\end{array}
$$

$$
\begin{array}{r}
-0.00002700 \\
-0.00001800 \\
-0.00001100 \\
-0.00000600 \\
0.00000700 \\
0.00000600 \\
0.00001900 \\
0.00001400 \\
0.00000800 \\
0.00002900 \\
0.88514000 \\
0.00003100 \\
-0.88506400 \\
-0.00000200 \\
0.00000300 \\
0.00001000 \\
-0.00000500 \\
0.00004100 \\
0.00001700 \\
-0.00000300
\end{array}
$$

\section{Truncated analogue of CuATSM}<smiles>CNC1=NN2C=CN3N=CS[C@@]32S1</smiles>

\section{M06/Def2TZVP Enthalpy= M06/Def2TZVP Free Energy=}

$\mathrm{O}^{2}$
$\mathrm{~S}$
$\mathrm{C}$
$\mathrm{N}$
$\mathrm{N}$
$\mathrm{C}$
$\mathrm{N}$
$\mathrm{N}$
$\mathrm{C}$
$\mathrm{S}$
$\mathrm{C}$
$\mathrm{H}$
$\mathrm{H}$
$\mathrm{H}$
$\mathrm{C}$
$\mathrm{N}$
$\mathrm{H}$
$\mathrm{Cu}$
$\mathrm{H}$
$\mathrm{H}$
$\mathrm{H}$

\section{Truncated analogue of CuATSM}<smiles>CNC1=NN2C=CN3N=CS[C@@]32S1</smiles>

$-2905.077046$ $-2905.133577$

$$
\begin{array}{r}
-1.25800100 \\
-2.35663100 \\
-2.04103300 \\
-0.73602300 \\
1.26494500 \\
1.84351500 \\
3.18865200 \\
3.52983500 \\
2.52732200 \\
-4.70667100 \\
-4.64033700 \\
-5.66956900 \\
-4.64031500 \\
-0.18331100 \\
-3.66128100 \\
-3.90468800 \\
0.60082300 \\
4.60294900 \\
1.84448400 \\
-0.77134100
\end{array}
$$

0.00000100
-0.00000300
-0.00000500
-0.00000400
-0.00000300
0.00000100
0.00000200
0.00000300
0.00000300
-0.00000400
-0.88257700
0.00001600
0.88254800
-0.00000500
0.00000000
0.00000000
0.00000200
0.00000700
-0.00000500
-0.00000700 


$\begin{array}{lrrr}02 & & \\ \mathrm{~S} & -1.23782500 & -1.64751900 & 0.00000300 \\ \mathrm{C} & -2.34701500 & -0.30534400 & 0.00000500 \\ \mathrm{~N} & -2.04085700 & 1.00536200 & 0.00000300 \\ \mathrm{~N} & -0.72563100 & 1.17901500 & 0.00000100 \\ \mathrm{C} & 1.26632100 & 2.36679200 & -0.00000100 \\ \mathrm{~N} & 1.84111200 & 1.20065900 & -0.00000100 \\ \mathrm{~N} & 3.19118400 & 1.06538700 & -0.00000200 \\ \mathrm{C} & 3.52149600 & -0.20346700 & -0.00000100 \\ \mathrm{~S} & 2.49660400 & -1.58448700 & -0.00000100 \\ \mathrm{C} & -4.70220500 & 0.41384000 & -0.00000400 \\ \mathrm{H} & -4.63117200 & 1.05340400 & -0.88290900 \\ \mathrm{H} & -5.66805500 & -0.08889700 & -0.00002100 \\ \mathrm{H} & -4.63119400 & 1.05338700 & 0.88291400 \\ \mathrm{C} & -0.17392700 & 2.36406900 & 0.00000000 \\ \mathrm{~N} & -3.65869300 & -0.59035500 & -0.00000300 \\ \mathrm{H} & -3.90590500 & -1.56664900 & -0.00000200 \\ \mathrm{Cu} & 0.59937500 & -0.33588000 & 0.00000100 \\ \mathrm{H} & 4.59347500 & -0.39429000 & -0.00000700 \\ \mathrm{H} & 1.85063100 & 3.28541800 & -0.00000300 \\ \mathrm{H} & -0.76794000 & 3.27441000 & 0.00000000\end{array}$

Truncated analogue of CuATSM<smiles>CNC1=NN2C=CN3N=CSC32S1</smiles>

$-2905.240634$ $-2905.298602$

$\mathrm{O}^{2}$
$\mathrm{~S}$
$\mathrm{C}$
$\mathrm{N}$
$\mathrm{N}$
$\mathrm{C}$
$\mathrm{N}$
$\mathrm{N}$
$\mathrm{C}$
$\mathrm{S}$
$\mathrm{C}$
$\mathrm{H}$
$\mathrm{H}$
$\mathrm{H}$
$\mathrm{C}$
$\mathrm{N}$
$\mathrm{H}$
$\mathrm{Cu}$
$\mathrm{H}$
$\mathrm{H}$
$\mathrm{H}$

$$
\begin{array}{r}
-1.27487600 \\
-2.36878200 \\
-2.05533800 \\
-0.74092300 \\
1.25193500 \\
1.84278300 \\
3.19394400 \\
3.55238900 \\
2.56479100 \\
-4.72267100 \\
-4.64860900 \\
-5.69097800 \\
-4.64867100 \\
-0.18797500 \\
-3.68452800 \\
-3.93919000 \\
0.60597900 \\
4.62923900 \\
1.82584700 \\
-0.78059500
\end{array}
$$

$$
\begin{array}{r}
0.00002200 \\
0.00003600 \\
0.00002100 \\
0.00000900 \\
-0.00000900 \\
-0.00001000 \\
-0.00002200 \\
-0.00001500 \\
-0.00001000 \\
-0.00002600 \\
-0.88332400 \\
-0.00006700 \\
0.88329600 \\
0.00000100 \\
-0.00000400 \\
-0.00001800 \\
0.00000300 \\
-0.00004500 \\
-0.00001800 \\
0.00000300
\end{array}
$$

\section{Truncated analogue of CuATSM}


<smiles>CNC1=NN2C=CN3N=CS[C@@]32S1</smiles>

\section{M06-L/Def2TZVP Enthalpy= $-2905.362795$ M06-L/Def2TZVP Free Energy= $-2905.420447$}

02
S
C
N
N
C
N
N
C
S
C
H
$\mathrm{H}$
$\mathrm{H}$
$\mathrm{C}$
$\mathrm{N}$
$\mathrm{H}$
$\mathrm{Cu}$
$\mathrm{H}$
$\mathrm{H}$
$\mathrm{H}$

$$
\begin{array}{r}
-1.27638000 \\
-2.36637700 \\
-2.05155700 \\
-0.74274300 \\
1.24824800 \\
1.84115600 \\
3.18790900 \\
3.54576500 \\
2.56545400 \\
-4.71416100 \\
-4.64257500 \\
-5.67956500 \\
-4.64257200 \\
-0.18752000 \\
-3.67738900 \\
-3.92985000 \\
0.60514800 \\
4.62054600 \\
1.81806800 \\
-0.77589900
\end{array}
$$$$
-1.65538000
$$$$
-0.30509800
$$$$
0.99910500
$$$$
1.17807100
$$$$
2.36382900
$$$$
1.21364200
$$$$
1.09448800
$$

$-0.16134000$

$-1.56568000$

0.42734800

1.06535100

$-0.06975800$

1.06537800

2.35416000

$-0.57810200$

$-1.54995600$

$-0.36658600$

$-0.32210100$

3. 28932800

3.26587700

0.00001200

0.00001700

0.00000700

0.00000200

$-0.00000700$

$-0.00000500$

$-0.00000900$

$-0.00000500$

$-0.00000300$

$-0.00001700$

$-0.88042000$

$-0.00000800$

0.88036500

$-0.00000300$

0.00000000

$-0.00001100$

0.00000300

$-0.00001600$

$-0.00001300$

$-0.00000300$

\section{Truncated analogue of CuATSM}<smiles>CNC1=NN2C=CN3N=CS[C@@]32S1</smiles>

\section{MN15/CBSB7 Enthalpy= MN15/CBSB7 Free Energy=}

$-2904.959057$ $-2905.014940$

$$
\begin{array}{rr}
-1.62669600 & -0.00000500 \\
-0.29877700 & 0.00000100 \\
1.00255100 & 0.00000400 \\
1.18297200 & 0.00000300 \\
2.34790500 & 0.00000300 \\
1.18687600 & -0.00000200 \\
1.03463000 & -0.00000200 \\
-0.22819600 & -0.00000700 \\
-1.58111700 & 0.00000100 \\
0.40917600 & 0.00000800 \\
1.04590400 & 0.88317800 \\
-0.09235700 & -0.00000300 \\
1.04592400 & -0.88314800 \\
2.35677300 & 0.00000300 \\
-0.59939600 & 0.00000100 \\
-1.58002200 & 0.00000100 \\
-0.32210500 & -0.00000200 \\
-0.44729500 & 0.00000800 \\
3.25452200 & 0.00000600
\end{array}
$$


Truncated analogue of CuATSM<smiles>CNC1=NN2C=CN3N=CS[C@@]32S1</smiles>

\section{MN15/CBSB7+ Enthalpy= MN15/CBSB7+ Free Energy=}

$\mathrm{O}^{2}{ }^{2}$
$\mathrm{C}$
$\mathrm{N}$
$\mathrm{N}$
$\mathrm{C}$
$\mathrm{N}$
$\mathrm{N}$
$\mathrm{C}$
$\mathrm{S}$
$\mathrm{C}$
$\mathrm{H}$
$\mathrm{H}$
$\mathrm{H}$
$\mathrm{C}$
$\mathrm{N}$
$\mathrm{H}$
$\mathrm{Cu}$
$\mathrm{H}$
$\mathrm{H}$
$\mathrm{H}$

$-2905.002187$ $-2905.058485$

$$
\begin{array}{r}
1.24652800 \\
2.35621300 \\
2.04635400 \\
0.72954000 \\
-1.28193200 \\
-1.84927200 \\
-3.20611800 \\
-3.53497700 \\
-2.49854900 \\
4.70988300 \\
4.63271900 \\
5.67571700 \\
4.63271000 \\
0.17839200 \\
3.66467700 \\
3.90844700 \\
-0.59897900 \\
-4.60314500 \\
-1.87309700 \\
0.76763200
\end{array}
$$

$$
\begin{array}{r}
-0.00000600 \\
0.00000000 \\
0.00000300 \\
0.00000300 \\
0.00000100 \\
-0.00000400 \\
-0.00000400 \\
0.00000400 \\
0.00000400 \\
0.00000500 \\
0.88373000 \\
-0.00000500 \\
-0.88370600 \\
0.00000300 \\
-0.00000100 \\
-0.00000200 \\
-0.00000200 \\
-0.00001000 \\
0.00000400 \\
0.00000400
\end{array}
$$

\section{Truncated analogue of CuATSM}<smiles>CNC1=NN2C=CN3N=CS[C@@]32S1</smiles>

\section{MN15/ def2TZVP Enthalpy= MN15/ def2TZVP Free Energy=}

\footnotetext{
02

$\mathrm{S}$

C

$\mathrm{N}$

$\mathrm{N}$

C

$\mathrm{N}$

$\mathrm{N}$

C

S

C

$\mathrm{H}$

$\mathrm{H}$

$\mathrm{H}$

C

$\mathrm{N}$
}

1.24662200

2. 35060800

2. 04271000

0.72964000

$-1.28003500$

$-1.84744800$

$-3.19995400$

$-3.52599400$

$-2.49528000$

4.69750200

4.61989800

5.66298600

4.61991900

0.17810900

3.65639500
$-2904.965426$ $-2905.021514$

$$
\begin{array}{r}
-1.63078100 \\
-0.29922200 \\
1.00014500 \\
1.18502700 \\
2.34669300 \\
1.19525100 \\
1.05180100 \\
-0.20603400 \\
-1.57052600 \\
0.41602600 \\
1.05159400 \\
-0.08379800 \\
1.05160300 \\
2.35238500 \\
-0.59194800
\end{array}
$$

-0.00001800
-0.00001100
-0.00000600
-0.00000300
0.00001100
0.00000700
0.00001500
0.00001000
0.00000100
0.00001900
0.88222400
0.00002700
-0.88218200
0.00000300
0.00000200 


$\begin{array}{lrrr}\mathrm{H} & 3.89943600 & -1.57103000 & 0.00000400 \\ \mathrm{Cu} & -0.59713500 & -0.34087500 & -0.00000400 \\ \mathrm{H} & -4.59380700 & -0.40442400 & 0.00001800 \\ \mathrm{H} & -1.87003500 & 3.25844300 & 0.00001800 \\ \mathrm{H} & 0.76649900 & 3.26287500 & 0.00000500\end{array}$

Truncated analogue of CuATSM<smiles>CNC1=NN2C=CN3N=CS[C@@]32S1</smiles>

\section{MN15-L/CBSB7 Enthalpy= MN15-L/CBSB7 Free Energy= \\ $-2904.823865$ $-2904.880900$}

$\mathrm{O}^{2}$
$\mathrm{~S}$
$\mathrm{C}$
$\mathrm{N}$
$\mathrm{N}$
$\mathrm{C}$
$\mathrm{N}$
$\mathrm{N}$
$\mathrm{C}$
$\mathrm{S}$
$\mathrm{C}$
$\mathrm{H}$
$\mathrm{H}$
$\mathrm{H}$
$\mathrm{C}$
$\mathrm{N}$
$\mathrm{H}$
$\mathrm{Cu}$
$\mathrm{H}$
$\mathrm{H}$
$\mathrm{H}$

$$
\begin{array}{r}
-1.64450700 \\
-0.30218300 \\
1.01464400 \\
1.17597100 \\
2.36591400 \\
1.18882900 \\
1.06208100 \\
-0.21286000 \\
-1.58864500 \\
0.41138300 \\
1.05654000 \\
-0.10402700 \\
1.05656000 \\
2.36897500 \\
-0.59692200 \\
-1.58329000 \\
-0.32955300 \\
-0.41174600 \\
3.28772700 \\
3.28609600
\end{array}
$$

\section{Truncated analogue of CuATSM}<smiles>CNC1=NN2C=CN3N=CS[C@@]32S1</smiles>

$$
\begin{array}{r}
-0.00000900 \\
-0.00000400 \\
0.00000000 \\
0.00000200 \\
0.00000400 \\
-0.00000300 \\
0.00000000 \\
-0.00000200 \\
0.00000500 \\
0.00001100 \\
0.89367800 \\
0.00000500 \\
-0.89364300 \\
0.00000200 \\
-0.00000100 \\
0.00000200 \\
-0.00000200 \\
0.00000900 \\
0.00001100 \\
0.00000100
\end{array}
$$

$-2904.873550$ $-2904.928056$

$$
\begin{array}{r}
-1.65301300 \\
-0.30122700 \\
1.01552200 \\
1.17859700 \\
2.36983000 \\
1.19999100 \\
1.08922700 \\
-0.18044600 \\
-1.57690800 \\
0.42168200 \\
1.06542800 \\
-0.09433000
\end{array}
$$




$\begin{array}{lrrr}\mathrm{H} & 4.67252300 & 1.06544300 & -0.89448400 \\ \mathrm{C} & 0.18485700 & 2.36879600 & 0.00000300 \\ \mathrm{~N} & 3.69339400 & -0.58863200 & 0.00000000 \\ \mathrm{H} & 3.93828800 & -1.57437600 & 0.00000400 \\ \mathrm{Cu} & -0.60708300 & -0.35682100 & -0.00000200 \\ \mathrm{H} & -4.65082800 & -0.35742200 & 0.00002600 \\ \mathrm{H} & -1.86166700 & 3.29812600 & 0.00001500 \\ \mathrm{H} & 0.78517200 & 3.28892800 & 0.00000200\end{array}$

Truncated analogue of CuATSM<smiles>CNC1=NN2C=CN3N=CS[C@@]32S1</smiles>

\section{MN15-L/def2TZVP Enthalpy= $\quad-2904.925206$ \\ MN15-L/def2TZVP Free Energy= $\quad-2904.979460$}

$\begin{array}{lrrr}02 & & \\ \mathrm{~S} & -1.26284900 & -1.64768400 & 0.00001300 \\ \mathrm{C} & -2.36524700 & -0.30323100 & 0.00001500 \\ \mathrm{~N} & -2.05998700 & 1.01151100 & 0.00000500 \\ \mathrm{~N} & -0.73684500 & 1.17728400 & -0.00000100 \\ \mathrm{C} & 1.26908900 & 2.36256900 & -0.00000900 \\ \mathrm{~N} & 1.84897400 & 1.19723700 & -0.00000500 \\ \mathrm{~N} & 3.20902300 & 1.08082800 & -0.00000800 \\ \mathrm{C} & 3.55118300 & -0.18770100 & -0.00000300 \\ \mathrm{~S} & 2.53781700 & -1.57523700 & -0.00000100 \\ \mathrm{C} & -4.73081700 & 0.41901800 & -0.00001500 \\ \mathrm{H} & -4.65528800 & 1.06238200 & -0.89212800 \\ \mathrm{H} & -5.70287600 & -0.09285500 & -0.00002000 \\ \mathrm{H} & -4.65530200 & 1.06239200 & 0.89209300 \\ \mathrm{C} & -0.18335500 & 2.36243100 & -0.00000600 \\ \mathrm{~N} & -3.68107600 & -0.59023000 & 0.00000100 \\ \mathrm{H} & -3.92488300 & -1.57442700 & -0.00000800 \\ \mathrm{Cu} & 0.60411900 & -0.35004500 & 0.00000300 \\ \mathrm{H} & 4.63548100 & -0.36516200 & -0.00001500 \\ \mathrm{H} & 1.85930500 & 3.28909600 & -0.00001600 \\ \mathrm{H} & -0.78113000 & 3.28169200 & -0.00000800\end{array}$

Peroxyl adduct derived from truncated CuATSM<smiles></smiles>

CBS-QB3 Enthalpy $=-3092.643600$

CBS-QB3 Free Energy $=-3092.711354$

B3LYP/CBSB7 Enthalpy = -3095.632866

B3LYP/CBSB7 Free Energy = -3095.700351

03

$\mathrm{S}$

C

$\mathrm{N}$

$\mathrm{N}$

C

$\mathrm{N}$

$\mathrm{N}$

C

$\begin{array}{rr}0.13686300 & -2.42733400 \\ 1.62399700 & -1.63688400 \\ 1.73912200 & -0.32491500 \\ 0.59703400 & 0.31535000 \\ -0.85986500 & 2.16377000 \\ -1.74867000 & 1.29854900 \\ -3.07993000 & 1.62522600 \\ -3.81118100 & 0.60022900\end{array}$

$-1.63688400$

$-0.32491500$

2.16377000

1.62522600

0.60022900
0.33823200

$-0.12466200$

$-0.41624200$

$-0.31483500$

$-0.68272600$

$-0.36941100$

$-0.36169100$

$-0.01730100$ 
$-1.00491400$

$-1.85705800$

$-1.35214000$

$-2.69137500$

$-1.14361200$

1.72883500

2.56305700

2.72879100

2.35062300

1.29257400

2.53670200

2.97341500

$-2.37696300$

$-3.34936700$

$-0.53262400$

0.79400100

3.18888700

1.92038700
0.38810200

$-0.55525600$

0.29683200

$-0.86166100$

$-1.37407700$

$-0.63682500$

0.35788400

0.02767100

1. 18503100

1. 41562400

0.91251200

2. 04264600

$-0.21486900$

0.03360600

0.07109400

0.01551000

$-0.93430000$

$-1.58443700$

\section{Peroxyl adduct derived from truncated CuATSM}<smiles>CNC1=NN2C(OC)CN3N=CS[C@]32S1</smiles>

\section{wB97X/CBSB7 Enthalpy= wB97X/CBSB7 Free Energy=}

${ }^{0}{ }^{3}$
$\mathrm{C}$
$\mathrm{C}$
$\mathrm{N}$
$\mathrm{N}$
$\mathrm{C}$
$\mathrm{N}$
$\mathrm{N}$
$\mathrm{C}$
$\mathrm{S}$
$\mathrm{C}$
$\mathrm{H}$
$\mathrm{H}$
$\mathrm{H}$
$\mathrm{C}$
$\mathrm{O}$
$\mathrm{O}$
$\mathrm{C}$
$\mathrm{H}$
$\mathrm{H}$
$\mathrm{H}$
$\mathrm{N}$
$\mathrm{H}$
$\mathrm{CU}$
$\mathrm{H}$
$\mathrm{H}$
$-3095.404472$ $-3095.470248$

\begin{abstract}
$-2.40553900$
$-1.60849100$

$-0.29350300$

0.32944200

2.18023100

1.29892000

1.63141700

0.59622900

$-1.01465900$

$-1.77075600$

$-1.11586400$

$-2.59333500$

$-1.18930400$

1.74306900

2.55627600

2.67686600

2.18712800

1.12244600

2.34423900

2.75900300

$-2.32832500$

$-3.30884400$

$-0.53528500$

0.78098800

3.21615000
\end{abstract}

0.28007300

$-0.12078100$

$-0.39246700$

$-0.30483500$

$-0.59927200$

$-0.32604700$

$-0.28784900$

0.02144000

0.35337900

$-0.49106500$

0.31107700

$-0.61519900$

$-1.41301400$

$-0.60717100$

0.36094800

0.00815100

1. 10539200

1. 26222200

0.81342400

2. 01156000

$-0.17919500$

0.03158800

0.04779600

0.07566600

$-0.79287500$ 
Peroxyl adduct derived from truncated CuATSM<smiles></smiles>

$\begin{array}{ll}\text { M06-2X/Def2TZVP Enthalpy }= & -3095.391622 \\ \text { M06-2X/Def2TZVP Free Energy }= & -3095.457667\end{array}$

$\begin{array}{lrrr}03 & & \\ \mathrm{~S} & 0.30998100 & -2.45104200 & 0.31407800 \\ \mathrm{C} & 1.71531000 & -1.55524200 & -0.13558400 \\ \mathrm{~N} & 1.75573200 & -0.23788700 & -0.43161100 \\ \mathrm{~N} & 0.59698000 & 0.34399500 & -0.31726600 \\ \mathrm{C} & -0.92341300 & 2.14670400 & -0.66795100 \\ \mathrm{~N} & -1.78642200 & 1.26303600 & -0.38004200 \\ \mathrm{~N} & -3.12040400 & 1.57103600 & -0.36640900 \\ \mathrm{C} & -3.83886400 & 0.54629800 & -0.02432500 \\ \mathrm{~S} & -3.36134000 & -1.05395000 & 0.38602300 \\ \mathrm{C} & 4.13127300 & -1.54354500 & -0.59704500 \\ \mathrm{H} & 4.37738100 & -0.74312700 & 0.10033500 \\ \mathrm{H} & 4.92118300 & -2.28984000 & -0.58449100 \\ \mathrm{H} & 4.05184100 & -1.11282700 & -1.59463000 \\ \mathrm{C} & 0.53499800 & 1.74791200 & -0.64359400 \\ \mathrm{O} & 1.10860700 & 2.59464700 & 0.32810200 \\ \mathrm{O} & 2.50870600 & 2.64624600 & 0.08593400 \\ \mathrm{C} & 3.13818300 & 2.21209800 & 1.27182400 \\ \mathrm{H} & 2.88119400 & 1.17468100 & 1.49041300 \\ \mathrm{H} & 4.20445800 & 2.30114600 & 1.06534500 \\ \mathrm{H} & 2.86836600 & 2.85752600 & 2.10921800 \\ \mathrm{~N} & 2.88962600 & -2.18990800 & -0.21704900 \\ \mathrm{H} & 2.88314400 & -3.17569200 & -0.01632600 \\ \mathrm{Cu} & -1.10724900 & -0.59646600 & 0.07518600 \\ \mathrm{H} & -4.90599700 & 0.74665000 & 0.00295400 \\ \mathrm{H} & -1.20548700 & 3.17096900 & -0.89566800 \\ \mathrm{H} & 1.02389900 & 1.94350100 & -1.60476300\end{array}$

Peroxyl adduct derived from truncated CuATSM<smiles></smiles>

M06-2X/CBSB7+ Enthalpy= M06-2X/CBSB7+ Free Energy=

03

$\mathrm{S}$

C

$\mathrm{N}$

$\mathrm{N}$

C

$\mathrm{N}$

$\mathrm{N}$

C

0.30317600
1.71492000
1.75623700
0.59173700
-0.92496000
-1.78802300
-3.12348300
-3.84533600

$-2.45118500$

$-1.55529100$

$-0.23666700$

0.34243800

2.15437900

1.26451700

1.57972000

0.55377400
0.31188200
-0.13421700
-0.43308900
-0.32243200
-0.66003800
-0.37434800
-0.35841000
-0.01952200 
$-1.05343900$

$-1.54451100$

$-0.72721800$

$-2.28737600$

$-1.13735800$

1.75045900

2.58876500

2.63828300

2.20800700

1.17037700

2.29695800

2.85901600

$-2.19176100$

$-3.18011700$

$-0.59753100$

0.75228700

3.18121900

1.94957600
0.38294200

$-0.59515800$

0.08788000

$-0.55927200$

$-1.60427900$

$-0.64466800$

0.33192500

0.08036000

1. 26722100

1. 48925900

1.05186200

2. 10287800

$-0.20771400$

$-0.01116400$

0.07217500

0.01133100

$-0.87976700$

$-1.60703500$

\section{Peroxyl adduct derived from truncated CuATSM}<smiles>CNC1=NN2[C@H](OC)CN3N=CS[C@]32S1</smiles>

M06-2X-D3/CBSB7 Enthalpy= M06-2X-D3/CBSB7 Free Energy=
03

S

C

N

$\mathrm{N}$

C

$\mathrm{N}$

$\mathrm{N}$

C

$\mathrm{S}$

C

$\mathrm{H}$

$\mathrm{H}$

$\mathrm{H}$

C

0

O

C

$\mathrm{H}$

$\mathrm{H}$

$\mathrm{N}$

$\mathrm{H}$

$\mathrm{Cu}$

$\mathrm{H}$

$\mathrm{H}$

\begin{abstract}
0.31576700
1. 72151500

1.75661500

0.59329400

$-0.94501000$

$-1.78962300$

$-3.13291500$

$-3.82734300$

$-3.29937100$

4.13017600

4.40288400

4. 91402500

4. 02181700

0.52172200

1.06086200

2.47267900

3.04901700

2. 76806100

4.12439600

2.75678600

2.89140800

2.88606100

$-1.08330300$

$-4.90067100$

$-1.25187300$
\end{abstract}

$-3095.222274$

$-3095.288096$
0.35414900
-0.14645100
-0.46336600
-0.32808000
-0.70779600
-0.39745000
-0.39122000
-0.03543900
0.39530200
-0.65200700
0.04884400
-0.67218500
-1.64209500
-0.66259500
0.32471400
0.13757100
1.33875200
1.52051300
1.17359600
2.18056200
-0.24240400
-0.02336600
0.07763900
-0.00817800
-0.95589500 
Peroxyl adduct derived from truncated CuATSM<smiles></smiles>

$\begin{array}{ll}\text { M06-2X/CBSB7 Enthalpy= } & -3095.220712 \\ \text { M06-2X/CBSB7 Free Energy }= & -3095.286572\end{array}$

$\begin{array}{lrrr}\text { O }^{3} & & \\ \mathrm{~S} & 0.31434800 & -2.41518400 & 0.35372300 \\ \mathrm{C} & 1.72072300 & -1.53163800 & -0.14582700 \\ \mathrm{~N} & 1.75653200 & -0.21799900 & -0.46215700 \\ \mathrm{~N} & 0.59329600 & 0.36119600 & -0.32717300 \\ \mathrm{C} & -0.94439200 & 2.14036400 & -0.70570400 \\ \mathrm{~N} & -1.78945100 & 1.24150100 & -0.39630700 \\ \mathrm{~N} & -3.13260800 & 1.52717200 & -0.38994500 \\ \mathrm{C} & -3.82752100 & 0.48918000 & -0.03526200 \\ \mathrm{~S} & -3.30024500 & -1.09968300 & 0.39402700 \\ \mathrm{C} & 4.12953300 & -1.53341400 & -0.65122100 \\ \mathrm{H} & 4.40174300 & -0.74154300 & 0.04832800 \\ \mathrm{H} & 4.91318200 & -2.28782200 & -0.66906600 \\ \mathrm{H} & 4.02241100 & -1.09177800 & -1.64238600 \\ \mathrm{C} & 0.52216700 & 1.76430100 & -0.66136700 \\ \mathrm{O} & 1.06276100 & 2.61835800 & 0.32540600 \\ \mathrm{O} & 2.47422200 & 2.65198100 & 0.13560700 \\ \mathrm{C} & 3.05308400 & 2.17723100 & 1.33538300 \\ \mathrm{H} & 2.77204500 & 1.13808700 & 1.51751600 \\ \mathrm{H} & 4.12810700 & 2.25361100 & 1.16767800 \\ \mathrm{H} & 2.76322700 & 2.80853800 & 2.17798600 \\ \mathrm{~N} & 2.89025000 & -2.17491200 & -0.24147900 \\ \mathrm{H} & 2.88397500 & -3.15849300 & -0.02348600 \\ \mathrm{Cu} & -1.08392200 & -0.57985900 & 0.07747600 \\ \mathrm{H} & -4.90077500 & 0.65650300 & -0.00799400 \\ \mathrm{H} & -1.25081900 & 3.15380900 & -0.95287000 \\ \mathrm{H} & 1.03143500 & 1.96533700 & -1.61119400\end{array}$

Peroxyl adduct derived from truncated CuATSM<smiles></smiles>

M06/CBSB7 Enthalpy $=\quad-3095.036192$

M06/CBSB7 Free Energy $=\quad-3095.103070$

$\begin{array}{lrrr}03 & & \\ \text { S } & 0.18117000 & -2.38446700 & 0.33215500 \\ \text { C } & 1.64086700 & -1.57130000 & -0.13738700 \\ \mathrm{~N} & 1.73452700 & -0.26398500 & -0.44222400 \\ \mathrm{~N} & 0.59120000 & 0.35250700 & -0.32148800 \\ \mathrm{C} & -0.89770900 & 2.15927100 & -0.67532500 \\ \mathrm{~N} & -1.76110100 & 1.27301800 & -0.36926400 \\ \mathrm{~N} & -3.09331100 & 1.56267600 & -0.35191000 \\ \mathrm{C} & -3.78550500 & 0.51513800 & -0.00903200\end{array}$


$-1.07059400$

$-1.71183600$

$-0.97105700$

$-2.51557100$

$-1.21683900$

1.75586200

2.59528100

2.67375400

2.18077400

1.12214300

2.29902200

2.77078000

$-2.27966300$

$-3.26058100$

$-0.53061500$

0.67344300

3.18374500

1.95210100
0.38492000

$-0.58520400$

0.14882900

$-0.63700400$

$-1.55826100$

$-0.64263300$

0.33932500

0.08631700

1.23662600

1. 40156500

1.02260900

2.12211100

$-0.21482000$

0.01032300

0.06443500

0.03179700

$-0.91329400$

$-1.59994000$

\section{Peroxyl adduct derived from truncated CuATSM}<smiles>CNC1=NN2C(OC)CN3N=CSC32S1</smiles>

M06/CBSB7+ Enthalpy $=\quad-3095.086533$ M06/CBSB7+ Free Energy $=-3095.153093$

\begin{abstract}
0.18045900
1.64260600

1.73672300

0.59052900

$-0.88021700$

$-1.76128000$

$-3.08554300$

$-3.80561500$

$-3.30797600$

4.06907300

4.33712200

4.83041500

4.04874000

0.56069400

1.17183300

2.56593400

3.26309000

3.05147900

4.31793300

3.02578000

2. 79125500

2. 72819400

$-1.11128800$

$-4.87854700$

$-1.14420900$
\end{abstract}

$$
\begin{array}{r}
-2.42092200 \\
-1.58990600 \\
-0.27819100 \\
0.33466200 \\
2.17139400 \\
1.29399600 \\
1.61218100 \\
0.57842500 \\
-1.02602000 \\
-1.70912400 \\
-0.89524700 \\
-2.48747000 \\
-1.30549800 \\
1.74319500 \\
2.56447500 \\
2.65817300 \\
2.20931500 \\
1.15326300 \\
2.33621500 \\
2.82220800 \\
-2.28651900 \\
-3.27481600 \\
-0.55341800 \\
0.76819700 \\
3.20849900
\end{array}
$$

$$
\begin{array}{r}
0.29016000 \\
-0.12479600 \\
-0.41370100 \\
-0.32017900 \\
-0.63034600 \\
-0.35056700 \\
-0.32401800 \\
0.00479000 \\
0.37503000 \\
-0.53661300 \\
0.14358900 \\
-0.47610200 \\
-1.55344500 \\
-0.62640100 \\
0.34766400 \\
0.03788600 \\
1.17632200 \\
1.37987300 \\
0.91796000 \\
2.05291800 \\
-0.17598200 \\
0.01624500 \\
0.05823200 \\
0.04921100 \\
-0.83840600
\end{array}
$$


Peroxyl adduct derived from truncated CuATSM<smiles></smiles>

\section{M06/Def2TZVP Enthalpy= $\quad-3095.241756$ M06/Def2TZVP Free Energy= $\quad-3095.308517$}

$\begin{array}{lrrr}03 & & \\ \mathrm{~S} & 0.16703900 & -2.42509400 & 0.27588200 \\ \mathrm{C} & 1.63149900 & -1.59712300 & -0.12274100 \\ \mathrm{~N} & 1.73156900 & -0.28604700 & -0.39691600 \\ \mathrm{~N} & 0.59294900 & 0.33267200 & -0.30780600 \\ \mathrm{C} & -0.86848200 & 2.17313000 & -0.60680800 \\ \mathrm{~N} & -1.75500600 & 1.30408800 & -0.33834200 \\ \mathrm{~N} & -3.07504100 & 1.62514700 & -0.30641100 \\ \mathrm{C} & -3.79880400 & 0.59501600 & 0.01074000 \\ \mathrm{~S} & -3.31696300 & -1.01300300 & 0.36327000 \\ \mathrm{C} & 4.05680700 & -1.72562600 & -0.50389900 \\ \mathrm{H} & 4.34601700 & -0.96212700 & 0.22207900 \\ \mathrm{H} & 4.80247700 & -2.51809600 & -0.49754800 \\ \mathrm{H} & 4.04120200 & -1.25923700 & -1.49086000 \\ \mathrm{C} & 0.56830900 & 1.73970100 & -0.60926600 \\ \mathrm{O} & 1.19226800 & 2.55736500 & 0.35309400 \\ \mathrm{O} & 2.57559800 & 2.66036100 & 0.01688100 \\ \mathrm{C} & 3.29349900 & 2.19819800 & 1.13193500 \\ \mathrm{H} & 3.08785100 & 1.14133700 & 1.32806800 \\ \mathrm{H} & 4.34249900 & 2.32603200 & 0.85902400 \\ \mathrm{H} & 3.07516800 & 2.79628700 & 2.02158600 \\ \mathrm{~N} & 2.77241400 & -2.29797200 & -0.17751300 \\ \mathrm{H} & 2.70273700 & -3.28188100 & 0.01894000 \\ \mathrm{Cu} & -1.11590400 & -0.55024200 & 0.05417200 \\ \mathrm{H} & -4.86874600 & 0.79262000 & 0.05728800 \\ \mathrm{H} & -1.12360900 & 3.21298600 & -0.80456300 \\ \mathrm{H} & 1.04630900 & 1.93187500 & -1.58205500 \\ & & & \end{array}$

Peroxyl adduct derived from truncated CuATSM<smiles></smiles>

M06-L/CBSB7 Enthalpy= $-3095.410530$ M06-L/CBSB7 Free Energy=-3095.477914

03

$\mathrm{S}$

C

$\mathrm{N}$

$\mathrm{N}$

C

$\mathrm{N}$

$\mathrm{N}$

C

$$
\begin{array}{r}
0.24115900 \\
1.67619000 \\
1.74316000 \\
0.57392800 \\
-0.93233400 \\
-1.78555600 \\
-3.12329300 \\
-3.80117900
\end{array}
$$

$$
\begin{array}{r}
-2.37008000 \\
-1.53575100 \\
-0.23005500 \\
0.36010400 \\
2.15366500 \\
1.25281600 \\
1.52473900 \\
0.46416300
\end{array}
$$

0.34280700
-0.16037600
-0.48729300
-0.36479200
-0.71979500
-0.38696100
-0.37520900
-0.00760000 
$-1.09772000$

$-1.62450800$

$-0.93443300$

$-2.41535100$

$-1.06197000$

1.76906400

2.59909800

2.65553800

2.16237500

1.11546100

2.24079100

2.77582400

$-2.22481400$

$-3.19317800$

$-0.55055900$

0.61431100

3.16720200

1.99168800
0.41517200

$-0.59985400$

0.17461500

$-0.72841200$

$-1.53081100$

$-0.67152800$

0.34910200

0.13231300

1. 31285700

1. 49323700

1.12323000

2. 18265000

$-0.24593600$

0.02819900

0.06678500

0.02975800

$-0.97942200$

$-1.60968700$

\section{Peroxyl adduct derived from truncated CuATSM}<smiles>CNC1=NN2[C@H](OC)CN3N=CS[C@@]32S1</smiles>

M06-L/CBSB7+ Enthalpy= M06-L/CBSB7+ Free Energy=

03

$\mathrm{S}$

C

N

$\mathrm{N}$

C

$\mathrm{N}$

$\mathrm{N}$

C

S

C

$\mathrm{H}$

$\mathrm{H}$

$\mathrm{H}$

C

O

O

C

$\mathrm{H}$

$\mathrm{H}$

$\mathrm{H}$

N

$\mathrm{H}$

$\mathrm{Cu}$

$\mathrm{H}$

$\mathrm{H}$

\begin{abstract}
0.24438400
1. 68186700

1. 74791700

0.57644900

$-0.91155600$

$-1.78472100$

$-3.11415600$

$-3.82331900$

$-3.30834700$

4.10195800

4.32609000

4.89975600

4. 06803600

0.53174600

1. 11230200

2.55109800

3. 19004100

2. 94763200

4. 25545000

2.94640300

2.85325900

2. 80816500

$-1.10591000$

$-4.89677800$

$-1.18947800$
\end{abstract}

$-3095.460012$ $-3095.527023$
$-2.40985600$
$-1.55343500$
$-0.24424400$
0.34221200
2.16569500
1.27429400
1.57666100
0.53214100
$-1.04995000$
$-1.63346400$
$-0.73785400$
$-2.36060600$
$-1.34521000$
1. 75485300
2.57444900
2.64333000
2.19953700
1.15464000
2.29195300
2.83633400
$-2.22413200$
$-3.21598100$
$-0.57777300$
0.71621300
3.19291900
0.31103700
$-0.13110000$
$-0.44746900$
$-0.35395200$
$-0.67966300$
$-0.37687200$
$-0.36106300$
$-0.00668300$
0.40076400
$-0.60411600$
$-0.02125900$
$-0.45915000$
$-1.65905400$
$-0.65452000$
0.35460800
0.08686700
1. 26266000
1. 48351700
1.03675500
2.11942200
$-0.17183200$
$-0.00195600$
0.06635900
0.02941800
$-0.91098200$ 
Peroxyl adduct derived from truncated CuATSM<smiles></smiles>

$\begin{array}{ll}\text { M06-L/Def2TZVP Enthalpy }= & -3095.598605 \\ \text { M06-L/Def2TZVP Free Energy }= & -3095.666042\end{array}$

$\begin{array}{lrrr}0{ }^{3} & & \\ \mathrm{~S} & 0.22234800 & -2.41744600 & 0.28805100 \\ \mathrm{C} & 1.66358200 & -1.56691900 & -0.13588100 \\ \mathrm{~N} & 1.73894300 & -0.25735600 & -0.43233700 \\ \mathrm{~N} & 0.57723500 & 0.33847100 & -0.34479100 \\ \mathrm{C} & -0.89698900 & 2.17240500 & -0.64377700 \\ \mathrm{~N} & -1.77709500 & 1.29124400 & -0.35497400 \\ \mathrm{~N} & -3.10187400 & 1.59929600 & -0.32584300 \\ \mathrm{C} & -3.81657500 & 0.55898700 & 0.01113200 \\ \mathrm{~S} & -3.32106000 & -1.03143100 & 0.38629000 \\ \mathrm{C} & 4.08971800 & -1.64158300 & -0.53654500 \\ \mathrm{H} & 4.37213300 & -0.87068600 & 0.18160200 \\ \mathrm{H} & 4.85300300 & -2.41398300 & -0.54159900 \\ \mathrm{H} & 4.04771300 & -1.17649500 & -1.52123800 \\ \mathrm{C} & 0.54003700 & 1.75010500 & -0.63767600 \\ \mathrm{O} & 1.14599700 & 2.56407700 & 0.35305800 \\ \mathrm{O} & 2.57792100 & 2.62144800 & 0.05083500 \\ \mathrm{C} & 3.23741100 & 2.18134700 & 1.21158300 \\ \mathrm{H} & 2.98642400 & 1.14551800 & 1.45240800 \\ \mathrm{H} & 4.29620400 & 2.25135600 & 0.96145400 \\ \mathrm{H} & 3.02767300 & 2.82805500 & 2.06694900 \\ \mathrm{~N} & 2.82450200 & -2.24316500 & -0.19118500 \\ \mathrm{H} & 2.78344700 & -3.22257200 & 0.02471000 \\ \mathrm{Cu} & -1.11382200 & -0.57205000 & 0.05811600 \\ \mathrm{H} & -4.88513500 & 0.75465500 & 0.05449300 \\ \mathrm{H} & -1.16148700 & 3.20459900 & -0.85763700 \\ \mathrm{H} & 1.03384300 & 1.96136200 & -1.59620500 \\ & & & \end{array}$

Peroxyl adduct derived from truncated CuATSM<smiles></smiles>

MN15/CBSB7 Enthalpy= MN15/CBSB7 Free Energy=

03

S

C

$\mathrm{N}$

$\mathrm{N}$

C

$\mathrm{N}$

$\mathrm{N}$

C

0.18992200
1.64529400
1.73271300
0.57988100
-0.91417200
-1.77093400
-3.10943800
-3.79689600
$-2.36962100$
$-1.56684600$
$-0.25627600$
0.35156500
2.16137200
1. 26418000
1.55596200
0.50483100

$$
\begin{array}{r}
-0.13588100 \\
-0.43233700 \\
-0.34479100 \\
-0.64377700 \\
-0.35497400 \\
-0.32584300 \\
0.01113200 \\
0.38629000 \\
-0.53654500 \\
0.18160200 \\
-0.54159900 \\
-1.52123800 \\
-0.63767600 \\
0.35305800 \\
0.05083500 \\
1.21158300 \\
1.45240800 \\
0.96145400 \\
2.06694900 \\
-0.19118500 \\
0.02471000 \\
0.05811600 \\
0.05449300 \\
-0.85763700 \\
-1.59620500
\end{array}
$$


$-1.07002000$

$-1.66428300$

$-0.89848500$

$-2.44527500$

$-1.19314900$

1.76029400

2.58514600

2. 63125800

2.15857600

1.11988800

2.22890500

2.79035800

$-2.26333900$

$-3.24673800$

$-0.53584200$

0.65554300

3.18266000

1.96044100
0.38321200

$-0.55532900$

0.17013800

$-0.56914100$

$-1.53801400$

$-0.64528500$

0.33748400

0.06882600

1. 23453900

1. 43602500

1. 01153400

2.09365300

$-0.20216700$

0.02163800

0.05498400

0.06803600

$-0.88014000$

$-1.60196700$

\section{Peroxyl adduct derived from truncated CuATSM}<smiles></smiles>

MN15/CBSB7+ Enthalpy= MN15/CBSB7+ Free Energy=

03

S

C

$\mathrm{N}$

$\mathrm{N}$

C

N

$\mathrm{N}$

C

S

C

$\mathrm{H}$

$\mathrm{H}$

$\mathrm{H}$

C

0

0

C

$\mathrm{H}$

$\mathrm{H}$

$\mathrm{H}$

N

$\mathrm{H}$

$\mathrm{Cu}$

$\mathrm{H}$

$\mathrm{H}$

\begin{abstract}
0.15708100
1. 62554100

1. 73093300

0.58042800

$-0.87976400$

$-1.76248100$

$-3.09102300$

$-3.81237800$

$-3.30037300$

4. 06112500

4.31657600

4.81906700

4.04806900

0.56281400

1.21143300

2. 59357600

3. 32027500

3. 08617100

4.36786500

3. 11536400

2.77301900

2. 70797500

$-1.12046600$

$-4.88169000$

$-1.14603900$

1.03096800
\end{abstract}

$-3095.050896$ $-3095.117104$

$-2.41003100$

$-1.60192600$

$-0.28539900$

0.32982200

2.18411100

1.30101300

1.63021400

0.59180100

$-1.01004500$

$-1.70889400$

$-0.92830500$

$-2.48950400$

$-1.26023900$

1.74450700

2.54419100

2. 61566200

2.19123600

1.15150900

2.28466700

2.83923100

$-2.30227500$

$-3.29097900$

$-0.54920300$

0.77931200

3.22274900

1.94220000
0.25984500

$-0.12109800$

$-0.40097100$

$-0.32209500$

$-0.58901000$

$-0.32164800$

$-0.27396500$

0.03724600

0.35764400

$-0.47472300$

0.24536900

$-0.44052600$

$-1.46952100$

$-0.61631400$

0.34893100

$-0.00870100$

1.12649100

1.36832600

0.83662500

1. 98210500

$-0.15791400$

0.03951000

0.04333100

0.09856300

$-0.76823600$

$-1.58987900$

Peroxyl adduct derived from truncated CuATSM 
<smiles></smiles>

MN15/ def2TZVP Enthalpy= MN15/ def2TZVP Free Energy=
$-3095.039313$ $-3095.105587$

O 3
S
C
N
N
C
N
N
C
S
C
H
H
H
C
$\mathrm{O}$
$\mathrm{O}$
$\mathrm{C}$
$\mathrm{H}$
$\mathrm{H}$
$\mathrm{H}$
$\mathrm{N}$
$\mathrm{H}$
$\mathrm{Cu}$
$\mathrm{H}$
$\mathrm{H}$
$\mathrm{H}$

$-2.40657100$

$-1.60217900$

$-0.28813900$

0.33038800

2. 17539300

1.29747200

1.62106500

0.58419200

$-1.00915500$

$-1.70833100$

$-0.93357900$

$-2.48823700$

$-1.25237700$

1.74053500

2.54787700

2.61482100

2.19894300

1.16189000

2.29033100

2. 85030500

$-2.30112700$

$-3.28768600$

$-0.54685100$

0.77477000

3. 21089900

1.93345500
0.26596000

$-0.12249500$

$-0.40361800$

$-0.31963200$

$-0.60183300$

$-0.32926400$

$-0.28409700$

0.03260100

0.36296600

$-0.48325800$

0.23987800

$-0.45881400$

$-1.47315700$

$-0.61927900$

0.34190100

$-0.00080700$

1. 14127000

1. 38978000

0.86435400

1. 99075800

$-0.16608800$

0.03498100

0.04651400

0.09068400

$-0.79065700$

$-1.59140800$

Peroxyl adduct derived from truncated CuATSM<smiles>CNC1=NN2[C@H](OC)C=NN3C=N[C@]2(S1)S3</smiles>

MN15-L/CBSB7 Enthalpy= MN15-L/CBSB7 Free Energy=

03

$\mathrm{S}$

C

$\mathrm{N}$

N

C

$\mathrm{N}$

$\mathrm{N}$

C

S

C

$\mathrm{H}$

$\mathrm{H}$

\begin{abstract}
0.32403900
1.73182600

1.75824200

0.56974900

$-0.99493500$

$-1.81693400$

$-3.17045100$

$-3.81663700$

$-3.18125100$

4.16416500

4.40575900

4.96613800
\end{abstract}

$-3094.885454$

$-3094.952021$
0.37174500
-0.18910100
-0.55426300
-0.38782800
-0.78155200
-0.41485500
-0.41168400
-0.01620800
0.43959900
-0.63549700
0.07880200
-0.61929500 


$\begin{array}{lrrr}\mathrm{H} & 4.10006700 & -1.05315000 & -1.64332000 \\ \mathrm{C} & 0.48187800 & 1.78826900 & -0.70916800 \\ \mathrm{O} & 0.96998900 & 2.63484300 & 0.32524600 \\ \mathrm{O} & 2.41555500 & 2.64712600 & 0.20227000 \\ \mathrm{C} & 2.90730300 & 2.16368400 & 1.43792700 \\ \mathrm{H} & 2.57398900 & 1.12485700 & 1.62869300 \\ \mathrm{H} & 4.00620900 & 2.19141100 & 1.32178700 \\ \mathrm{H} & 2.60627700 & 2.82141900 & 2.27658300 \\ \mathrm{~N} & 2.90941500 & -2.15199300 & -0.27954200 \\ \mathrm{H} & 2.90887600 & -3.12040900 & 0.02485500 \\ \mathrm{Cu} & -1.04519000 & -0.55821800 & 0.07331200 \\ \mathrm{H} & -4.91028000 & 0.47981000 & 0.02094200 \\ \mathrm{H} & -1.34295500 & 3.12892300 & -1.07228400 \\ \mathrm{H} & 1.03574200 & 2.01929700 & -1.64565100\end{array}$

\section{Peroxyl adduct derived from truncated CuATSM}<smiles></smiles>

MN15-L/CBSB7+ Enthalpy $=-3094.939039$

\section{MN15-L/CBSB7+ Free Energy $=\quad-3095.005535$}

$\begin{array}{lrrr}03 & & \\ \mathrm{~S} & 0.24971900 & -2.40818400 & 0.29219000 \\ \mathrm{C} & 1.69057800 & -1.55195000 & -0.15298700 \\ \mathrm{~N} & 1.75314900 & -0.23694100 & -0.48069200 \\ \mathrm{~N} & 0.56799000 & 0.34116700 & -0.37318500 \\ \mathrm{C} & -0.93485200 & 2.17066600 & -0.67964500 \\ \mathrm{~N} & -1.79940700 & 1.26263500 & -0.36681000 \\ \mathrm{~N} & -3.13820700 & 1.56936900 & -0.33534100 \\ \mathrm{C} & -3.83839700 & 0.51121000 & 0.02228300 \\ \mathrm{~S} & -3.29128700 & -1.07318300 & 0.40983500 \\ \mathrm{C} & 4.13994200 & -1.59235500 & -0.53756700 \\ \mathrm{H} & 4.35358600 & -0.74403500 & 0.13944000 \\ \mathrm{H} & 4.93712900 & -2.34572500 & -0.44249200 \\ \mathrm{H} & 4.12086300 & -1.20492600 & -1.57259600 \\ \mathrm{C} & 0.52540800 & 1.76338300 & -0.67177900 \\ \mathrm{O} & 1.10893400 & 2.57629700 & 0.33907300 \\ \mathrm{O} & 2.53860900 & 2.61701000 & 0.07879900 \\ \mathrm{C} & 3.15721200 & 2.20842300 & 1.28397800 \\ \mathrm{H} & 2.87169800 & 1.17186600 & 1.54956900 \\ \mathrm{H} & 4.23911900 & 2.26029400 & 1.06352100 \\ \mathrm{H} & 2.91354100 & 2.89933600 & 2.11423200 \\ \mathrm{~N} & 2.87017900 & -2.22026700 & -0.18916600 \\ \mathrm{H} & 2.83801200 & -3.21059900 & 0.03282300 \\ \mathrm{Cu} & -1.09958700 & -0.57294500 & 0.05630800 \\ \mathrm{H} & -4.92400600 & 0.68729000 & 0.07459200 \\ \mathrm{H} & -1.23294100 & 3.20647200 & -0.90287600 \\ \mathrm{H} & 1.04051400 & 1.98282400 & -1.63384700 \\ & & & \end{array}$

Peroxyl adduct derived from truncated CuATSM<smiles></smiles> 
MN15-L/def2TZVP Enthalpy=
MN15-L/def2TZVP Free Energy=

03

$\mathrm{S}$

C

$\mathrm{N}$

$\mathrm{N}$

C

$\mathrm{N}$

$\mathrm{N}$

C

S

C

$\mathrm{H}$

$\mathrm{H}$

$\mathrm{H}$

C

O

O

C

$\mathrm{H}$

$\mathrm{H}$

$\mathrm{H}$

N

$\mathrm{H}$

$\mathrm{Cu}$

$\mathrm{H}$

$\mathrm{H}$

$\mathrm{H}$

$$
\begin{array}{r}
0.24674900 \\
1.68322700 \\
1.75142100 \\
0.57218500 \\
-0.93286200 \\
-1.79452600 \\
-3.13291400 \\
-3.82605300 \\
-3.27787200 \\
4.12460200 \\
4.34538800 \\
4.91712600 \\
4.10429000 \\
0.52753700 \\
1.10454500 \\
2.53563400 \\
3.13707900 \\
2.84157000 \\
4.22029500 \\
2.89407000 \\
2.85519200 \\
2.82285200 \\
-1.09334700 \\
-4.91015400 \\
-1.23139400 \\
1.03886800
\end{array}
$$

$-3095.008466$

$-3095.074941$

\section{Aminyl radical derived from truncated CuATSM}

0.29474300

$-0.15486200$

$-0.47647000$

$-0.36214800$

$-0.68143500$

$-0.36969000$

$-0.34029200$

0.01793700

0.40934900

$-0.53654400$

0.16177000

$-0.46713900$

$-1.55733000$

$-0.66814800$

0.33176500

0.07766000

1.28689300

1.55687000

1. 08011700

2.11253300

$-0.20557600$

0.03154000

0.05904900

0.06675000

$-0.91000700$

$-1.63220500$<smiles>CNC1=NN2C=CN3N=CSC32S1</smiles>

$\begin{array}{ll}\text { CBS-QB3 Enthalpy }=-2901.999461 & \text { CBS-QB3 Free Energy }=-2902.055143 \\ \text { B3LYP/CBSB7 Enthalpy }=-2904.738880 & \text { B3LYP/CBSB7 Free Energy }=-2904.794364\end{array}$

03

$\begin{array}{lr}\text { S } & -1.21116200 \\ \mathrm{C} & -2.44920200 \\ \mathrm{~N} & -2.04000200 \\ \mathrm{~N} & -0.78544000 \\ \mathrm{C} & 1.18098300 \\ \mathrm{~N} & 1.78111700 \\ \mathrm{~N} & 3.10077100 \\ \mathrm{C} & 3.50818100 \\ \mathrm{~S} & 2.52763600 \\ \mathrm{C} & -4.73609800 \\ \mathrm{H} & -4.39270100 \\ \mathrm{H} & -5.36895300 \\ \mathrm{H} & -5.36892400 \\ \mathrm{C} & -0.20954100 \\ \mathrm{~N} & -3.68271300 \\ \mathrm{Cu} & 0.55700500 \\ \mathrm{H} & 4.58480900 \\ \mathrm{H} & 1.77393000 \\ \mathrm{H} & -0.82697800\end{array}$

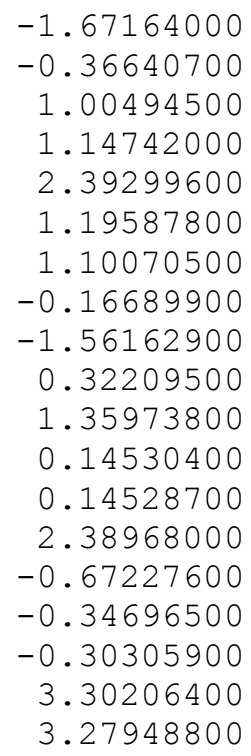

$$
\begin{array}{r}
-0.00003000 \\
0.00000400 \\
0.00001100 \\
-0.00000600 \\
-0.00000700 \\
-0.00001500 \\
0.00001700 \\
0.00003900 \\
0.00004000 \\
0.00004800 \\
0.00005400 \\
-0.87578200 \\
0.87589700 \\
-0.00000800 \\
0.00002100 \\
-0.00003700 \\
0.00006500 \\
0.00000500 \\
-0.00000400
\end{array}
$$


Aminyl radical derived from truncated CuATSM

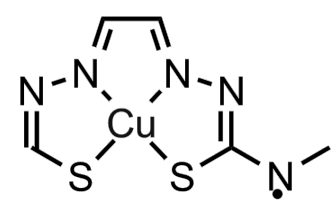

\section{wB97X/CBSB7 Enthalpy= wB97X/CBSB7 Free Energy=}

$-2904.546130$ $-2904.600961$
$\mathrm{O}^{3}$
$\mathrm{~S}$
$\mathrm{C}$
$\mathrm{N}$
$\mathrm{N}$
$\mathrm{C}$
$\mathrm{N}$
$\mathrm{N}$
$\mathrm{C}$
$\mathrm{S}$
$\mathrm{C}$
$\mathrm{H}$
$\mathrm{H}$
$\mathrm{H}$
$\mathrm{C}$
$\mathrm{N}$
$\mathrm{C}$
$\mathrm{H}$
$\mathrm{H}$
$\mathrm{H}$

Aminyl radical derived from truncated CuATSM

$$
\begin{array}{r}
-1.19718000 \\
-2.42690300 \\
-2.01725200 \\
-0.77908300 \\
1.17171900 \\
1.76741400 \\
3.07732400 \\
3.47613100 \\
2.49398400 \\
-4.69339100 \\
-4.33380200 \\
-5.32544500 \\
-5.32542800 \\
-0.20739500 \\
-3.65747100 \\
0.55471700 \\
4.55337600 \\
1.77630000 \\
-0.83814600
\end{array}
$$

\section{M06-2X/Def2TZVP Enthalpy= M06-2X/Def2TZVP Free Energy=}

$$
\begin{array}{r}
-0.00001000 \\
0.00000200 \\
0.00000100 \\
-0.00000300 \\
-0.00001000 \\
-0.00000900 \\
-0.00000400 \\
0.00000900 \\
0.00001600 \\
0.00002100 \\
0.00001800 \\
-0.87670600 \\
0.87676100 \\
-0.00000800 \\
0.00001100 \\
-0.00000700 \\
0.00000900 \\
-0.00001100 \\
-0.00000800
\end{array}
$$

03

$\mathrm{S}$

C

$\mathrm{N}$

$\mathrm{N}$

C

$\mathrm{N}$

$\mathrm{N}$

C

S

C

$\mathrm{H}$

$\mathrm{H}$

$\mathrm{H}$

C

$\mathrm{N}$

$\mathrm{Cu}$

$\mathrm{H}$

$\mathrm{H}$

$\mathrm{H}$

$$
\begin{array}{r}
-1.28331400 \\
-2.46959900 \\
-2.04312500 \\
-0.81105500 \\
1.13306800 \\
1.76298900 \\
3.06789400 \\
3.54043100 \\
2.66485300 \\
-4.72651700 \\
-4.35579200 \\
-5.35948900 \\
-5.35948800 \\
-0.23946300 \\
-3.70636200 \\
0.55787800 \\
4.62274800 \\
1.71780900 \\
-0.86877800
\end{array}
$$

$-2904.542835$ $-2904.598365$

$$
\begin{array}{r}
-1.70920300 \\
-0.39159100 \\
0.99082700 \\
1.12987200 \\
2.40047000 \\
1.21140200 \\
1.16429400 \\
-0.08360100 \\
-1.52008900 \\
0.37512600 \\
1.39931500 \\
0.21410600 \\
0.21410300 \\
2.38637200 \\
-0.64683700 \\
-0.40205400 \\
-0.14511500 \\
3.31313200 \\
3.26514500
\end{array}
$$


Aminyl radical derived from truncated CuATSM

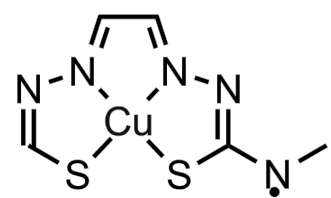

M06-2X/CBSB7+ Enthalpy= M06-2X/CBSB7+ Free Energy=
$-2904.434265$ $-2904.490766$
-0.00001400
0.00000000
-0.00000200
0.00000100
-0.00000800
-0.00001000
-0.00000200
0.00000900
0.00001700
0.00002300
0.00001700
-0.87570900
0.87577300
-0.00000400
0.00001100
-0.00000900
0.00001500
-0.00000800
-0.00000300

\section{Aminyl radical derived from truncated CuATSM}<smiles>CNC1=NN2C=CN3N=CS[C@@]32S1</smiles>

\section{M06-2X-D3/CBSB7 Enthalpy= M06-2X-D3/CBSB7 Free Energy=} 03

S
C
N
N
C
N
N
C
S
C
H
H
H
C
N
Cu
H
H
H

-1.25209100
-2.45734000
-2.03745500
-0.79969200
1.14555400
1.76336600
3.07233000
3.52253000
2.60928800
-4.71515300
-4.34295100
-5.34837300
-5.34835500
-0.23161100
-3.69406100
0.55625400
4.60348300
1.73874700
-0.86438800

$-2904.395290$ $-2904.450461$

$$
\begin{array}{r}
-1.70488600 \\
-0.39257300 \\
0.99170500 \\
1.13017600 \\
2.40197400 \\
1.20460600 \\
1.14513000 \\
-0.11183100 \\
-1.53401700 \\
0.36813500 \\
1.39310200 \\
0.20554400 \\
0.20554300 \\
2.39078800 \\
-0.65838700 \\
-0.37897500 \\
-0.20155400 \\
3.31019800 \\
3.26831700
\end{array}
$$

$-0.00001100$

0.00000100

0.00000100

$-0.00000300$

$-0.00000800$

$-0.00000800$

$-0.00000300$

0.00000700

0.00001500

0.00002200

0.00001900

$-0.87558600$

0.87564200

$-0.00000600$

0.00001100

$-0.00000800$

0.00001100

$-0.00000900$

$-0.00000600$ 
Aminyl radical derived from truncated CuATSM

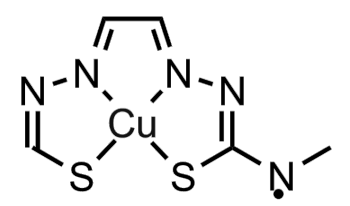

M06-2X/CBSB7 Enthalpy= M06-2X/CBSB7 Free Energy=
$-2904.394524$

$-2904.449689$

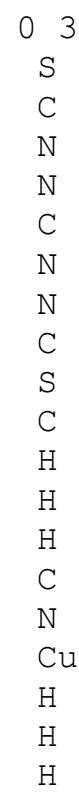

$$
\begin{array}{r}
-1.25204000 \\
-2.45732000 \\
-2.03746100 \\
-0.79967000 \\
1.14565600 \\
1.76342600 \\
3.07238200 \\
3.52246100 \\
2.60908500 \\
-4.71522700 \\
-4.34316400 \\
-5.34844400 \\
-5.34842600 \\
-0.23152200 \\
-3.69401400 \\
0.55628800 \\
4.60339400 \\
1.73889200 \\
-0.86423600
\end{array}
$$$$
-1.70469900
$$$$
-0.39247300
$$$$
0.99178100
$$$$
1.13017900
$$$$
2.40185400
$$$$
1.20451800
$$$$
1.14499300
$$$$
-0.11200000
$$$$
-1.53408100
$$$$
0.36795100
$$$$
1.39296900
$$$$
0.20524900
$$$$
0.20524800
$$$$
2.39070500
$$$$
-0.65841800
$$$$
-0.37886300
$$$$
-0.20184400
$$$$
3.31003200
$$

3.26826200

$$
\begin{array}{r}
-0.00001100 \\
0.00000100 \\
0.00000100 \\
-0.00000300 \\
-0.00000800 \\
-0.00000800 \\
-0.00000300 \\
0.00000700 \\
0.00001500 \\
0.00002200 \\
0.00001900 \\
-0.87557200 \\
0.87562900 \\
-0.00000600 \\
0.00001100 \\
-0.00000800 \\
0.00001200 \\
-0.00000900 \\
-0.00000600
\end{array}
$$

\section{Aminyl radical derived from truncated CuATSM}<smiles>CNC1=NN2C=CN3N=CS[C@@]32S1</smiles>

\section{M06/CBSB7 Enthalpy=} M06/CBSB7 Free Energy= 03

$\begin{array}{lr}\text { S } & -1.19118900 \\ \mathrm{C} & -2.42632500 \\ \mathrm{~N} & -2.02336600 \\ \mathrm{~N} & -0.77635300 \\ \mathrm{C} & 1.18731400 \\ \mathrm{~N} & 1.77642200 \\ \mathrm{~N} & 3.08873200 \\ \mathrm{C} & 3.47106100 \\ \mathrm{~S} & 2.47794800 \\ \mathrm{C} & -4.70113000 \\ \mathrm{H} & -4.36088200 \\ \mathrm{H} & -5.33799800 \\ \mathrm{H} & -5.33793600 \\ \mathrm{C} & -0.20073600 \\ \mathrm{~N} & -3.65315700 \\ \mathrm{Cu} & 0.55397800 \\ \mathrm{H} & 4.54827800 \\ \mathrm{H} & 1.78910200 \\ \mathrm{H} & -0.82111600\end{array}$

$-2904.244312$ $-2904.299691$

$$
\begin{array}{rr}
-1.65933700 & -0.00003100 \\
-0.36361400 & -0.00003900 \\
1.00996800 & -0.00001700 \\
1.15028300 & -0.00001200 \\
2.38569400 & -0.00000500 \\
1.19068800 & -0.00000300 \\
1.08297800 & 0.00001400 \\
-0.18889100 & 0.00003100 \\
-1.56503700 & 0.00003700 \\
0.31073600 & 0.00004700 \\
1.35197500 & 0.00003900 \\
0.13700100 & -0.87484100 \\
0.13699400 & 0.87497800 \\
2.38784400 & -0.00001200 \\
-0.67231400 & 0.00000300 \\
-0.33737400 & -0.00001200 \\
-0.34459600 & 0.00004500 \\
3.29199400 & 0.00000200 \\
3.27862300 & -0.00001600
\end{array}
$$

\section{Aminyl radical derived from truncated CuATSM}




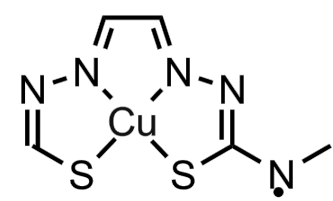

M06/CBSB7+ Enthalpy=

M06/CBSB7+ Free Energy=
$-2904.291839$ $-2904.348054$

$0^{3}$
S
C
N
N
C
N
N
C
S
C
H
H
H
C
N
Cu
H
H
H

$$
\begin{array}{r}
-1.22434800 \\
-2.44422800 \\
-2.03546500 \\
-0.78907400 \\
1.17551700 \\
1.77716400 \\
3.08982900 \\
3.49833300 \\
2.53973300 \\
-4.72093000 \\
-4.37975100 \\
-5.35749500 \\
-5.35746200 \\
-0.21150900 \\
-3.67266300 \\
0.55719800 \\
4.57937200 \\
1.76909600 \\
-0.83030100
\end{array}
$$$$
-1.66612100
$$$$
-0.35881300
$$$$
\text { 1. } 01262100
$$$$
\text { 1. } 15085400
$$$$
2.38892300
$$$$
1.19972000
$$$$
1.10990100
$$$$
-0.15425000
$$$$
-1.55212300
$$$$
0.31857500
$$$$
1.35938900
$$$$
0.14549900
$$$$
0.14550200
$$$$
\text { 2. } 38645300
$$$$
-0.66404300
$$$$
-0.36560000
$$$$
-0.28448100
$$$$
3.30100700
$$

3. 27867200

$$
\begin{array}{r}
-0.00002500 \\
-0.00000600 \\
-0.00000800 \\
-0.00000100 \\
-0.00000800 \\
-0.00001100 \\
-0.00000100 \\
0.00001700 \\
0.00002900 \\
0.00003000 \\
0.00002200 \\
-0.87507600 \\
0.87516000 \\
-0.00000700 \\
0.00001200 \\
-0.00001000 \\
0.00002100 \\
-0.00000500 \\
-0.00001000
\end{array}
$$

\section{Aminyl radical derived from truncated CuATSM}<smiles>CNC1=NN2C=CN3N=CS[C@@]32S1</smiles>

\section{M06/Def2TZVP Enthalpy= \\ M06/Def2TZVP Free Energy=}

\section{$-2904.425495$ \\ $-2904.481260$}

\footnotetext{
03

$\mathrm{S}$

C

$\mathrm{N}$

$\mathrm{N}$

C

$\mathrm{N}$

$\mathrm{N}$

C

S

C

$\mathrm{H}$

$\mathrm{H}$

$\mathrm{H}$

C

$\mathrm{N}$

$\mathrm{Cu}$

$\mathrm{H}$

$\mathrm{H}$
}

$$
\begin{array}{r}
-1.22810400 \\
-2.44283500 \\
-2.03195300 \\
-0.79125200 \\
1.17205400 \\
1.77696400 \\
3.08518100 \\
3.49400200 \\
2.54470100 \\
-4.71672700 \\
-4.37971400 \\
-5.35208800 \\
-5.35207500 \\
-0.21161900 \\
-3.66914000 \\
0.55701600 \\
4.57408200 \\
1.76116700 \\
-0.82823200
\end{array}
$$

$$
\begin{array}{r}
-0.00000300 \\
0.00000200 \\
-0.00000200 \\
-0.00000200 \\
-0.00000800 \\
-0.00000500 \\
-0.00000400 \\
0.00000400 \\
0.00000400 \\
0.00001500 \\
0.00000800 \\
-0.87332100 \\
0.87336300 \\
-0.00000700 \\
0.00001200 \\
-0.00000200 \\
-0.00000500 \\
-0.00001000 \\
-0.00000900
\end{array}
$$

Aminyl radical derived from truncated CuATSM 


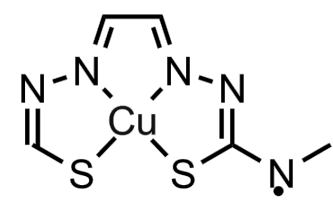

M06-L/CBSB7 Enthalpy=

$-2904.548943$

M06-L/CBSB7 Free Energy= $=2904.604944$

$\mathrm{O}^{3}$
$\mathrm{~S}$
$\mathrm{C}$
$\mathrm{N}$
$\mathrm{N}$
$\mathrm{C}$
$\mathrm{N}$
$\mathrm{N}$
$\mathrm{C}$
$\mathrm{S}$
$\mathrm{C}$
$\mathrm{H}$
$\mathrm{H}$
$\mathrm{H}$
$\mathrm{C}$
$\mathrm{N}$
$\mathrm{Cu}$
$\mathrm{H}$
$\mathrm{H}$
$\mathrm{H}$

$$
\begin{array}{r}
-1.19739500 \\
-2.43840600 \\
-2.04140400 \\
-0.77867100 \\
1.18872900 \\
1.77545400 \\
3.10331500 \\
3.48668300 \\
2.48605600 \\
-4.71421900 \\
-4.37410200 \\
-5.35188000 \\
-5.35184000 \\
-0.20260700 \\
-3.66630000 \\
0.56044000 \\
4.56312700 \\
1.78931300 \\
-0.81377800
\end{array}
$$$$
-1.64370800
$$$$
-0.34915900
$$$$
\text { 1. } 01762700
$$$$
1.15652400
$$$$
2.38369700
$$$$
1.18296000
$$$$
1.08529000
$$$$
-0.18508200
$$$$
-1.56343300
$$$$
0.29970800
$$$$
1.33991700
$$$$
0.12737600
$$$$
0.12737400
$$$$
2.39083800
$$$$
-0.68605000
$$$$
-0.34663200
$$$$
-0.33951700
$$$$
3.28989500
$$

3.28705400

$$
\begin{array}{r}
-0.00003800 \\
-0.00001300 \\
-0.00000900 \\
-0.00000500 \\
-0.00000700 \\
-0.00001000 \\
0.00000400 \\
0.00002300 \\
0.00004000 \\
0.00003800 \\
0.00003200 \\
-0.87333600 \\
0.87344200 \\
-0.00001000 \\
0.00001100 \\
-0.00001100 \\
0.00003300 \\
0.00000100 \\
-0.00001600
\end{array}
$$

\section{Aminyl radical derived from truncated CuATSM}<smiles>CNC1=NN2C=CN3N=CS[C@@]32S1</smiles>

\section{M06-L/CBSB7+ Enthalpy= M06-L/CBSB7+ Free Energy=}

$0^{3} 3$
$\mathrm{~S}$
$\mathrm{C}$
$\mathrm{N}$
$\mathrm{N}$
$\mathrm{C}$
$\mathrm{N}$
$\mathrm{N}$
$\mathrm{C}$
$\mathrm{S}$
$\mathrm{C}$
$\mathrm{H}$
$\mathrm{H}$
$\mathrm{H}$
$\mathrm{C}$
$\mathrm{N}$
$\mathrm{Cu}$
$\mathrm{H}$
$\mathrm{H}$
$\mathrm{H}$

Aminyl radical derived from truncated CuATSM
$-2904.596318$ $-2904.652947$

$$
\begin{array}{rr}
-1.23676400 & -1.65408800 \\
-2.45829000 & -0.34647700 \\
-2.05506200 & 1.01790200 \\
-0.79310700 & 1.15471600 \\
1.17372000 & 2.38750800 \\
1.77562200 & 1.19439800 \\
3.10288800 & 1.11918400 \\
3.51743700 & -0.14217100 \\
2.55900700 & -1.54632000 \\
-4.73385000 & 0.31429800 \\
-4.38902500 & 1.35291800 \\
-5.37206500 & 0.14502600 \\
-5.37202800 & 0.14502300 \\
-0.21657600 & 2.38711600 \\
-3.68991200 & -0.67469600 \\
0.56429200 & -0.38058600 \\
4.59832000 & -0.26589700 \\
1.76443900 & 3.30048600 \\
-0.82764200 & 3.28379200
\end{array}
$$

-0.00004100
-0.00000900
-0.00000800
0.00000100
-0.00000700
-0.00001200
-0.00000200
0.00001900
0.00004300
0.00003500
0.00003000
-0.87345900
0.87355500
-0.00000800
0.00001000
-0.00000900
0.00002700
-0.00000200
-0.00001500 0 (2) (2) 


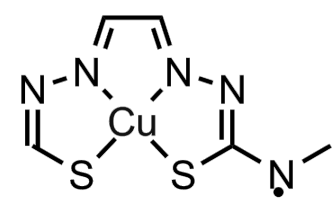

M06-L/Def2TZVP Enthalpy=

M06-L/Def2TZVP Free Energy=

$$
\begin{array}{r}
-1.23760200 \\
-2.45546800 \\
-2.05082100 \\
-0.79579000 \\
1.16888200 \\
1.77412600 \\
3.09603000 \\
3.51155900 \\
2.56404000 \\
-4.72872400 \\
-4.38963800 \\
-5.36484600 \\
-5.36483400 \\
-0.21673800 \\
-3.68422100 \\
0.56286500 \\
4.59063000 \\
1.75458900 \\
-0.82432800
\end{array}
$$

$-2904.717674$

$-2904.773972$
0.00000100

$-0.00000100$

$-0.00000100$

$-0.00000200$

$-0.00000900$

$-0.00000800$

$-0.00000800$

0.00000000

0.00000500

0.00001400

0.00001200

$-0.87104300$

0.87107900

$-0.00000700$

0.00000700

$-0.00000100$

$-0.00000500$

$-0.00001200$

$-0.00000900$

\section{Aminyl radical derived from truncated CuATSM}<smiles>CNC1=NN2C=CN3N=CS[C@@]32S1</smiles>

\section{MN15/CBSB7 Enthalpy= MN15/CBSB7 Free Energy=}

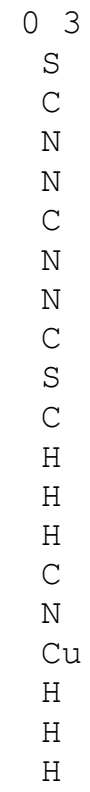

\section{$-2904.304081$ $-2904.359255$}

-1.19104400
-2.42253600
-2.02116000
-0.77421700
1.18476400
1.77758200
3.08951800
3.47529300
2.47336300
-4.70041400
-4.34779300
-5.33413700
-5.33415200
-0.19934900
-3.65583400
0.55339700
4.54894300
1.79028900
-0.82652600

$-1.64933000$

$-0.37643200$

0.99854400

1.13838100

2.38772100

1.18367900

1.07758300

$-0.19850600$

$-1.55240600$

0.32160900

1.35555100

0.15154500

0.15154100

2.38861900

$-0.67517700$

$-0.33903900$

$-0.35629900$

3.28792000

3.27050400
0.00000900

0.00002600

0.00002200

0.00000700

$-0.00001800$

$-0.00002100$

$-0.00002400$

$-0.00001000$

0.00001100

0.00000300

0.00000900

$-0.87472300$

0.87471800

$-0.00000500$

0.00000900

$-0.00000800$

$-0.00001200$

$-0.00002500$

$-0.00000100$

Aminyl radical derived from truncated CuATSM 


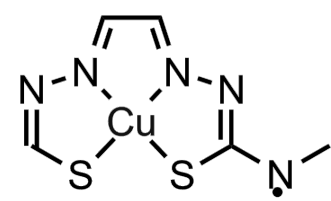

MN15/CBSB7+ Enthalpy=

MN15/CBSB7+ Free Energy=

$0^{3}{ }^{3}$
C
N
$\mathrm{N}$
$\mathrm{C}$
$\mathrm{N}$
$\mathrm{N}$
$\mathrm{C}$
$\mathrm{S}$
$\mathrm{C}$
$\mathrm{H}$
$\mathrm{H}$
$\mathrm{H}$
$\mathrm{C}$
$\mathrm{N}$
$\mathrm{Cu}$
$\mathrm{H}$
$\mathrm{H}$
$\mathrm{H}$
$-2904.349095$

$-2904.405103$
0.00000600

0.00000900

0.00000700

0.00000500

$-0.00001200$

$-0.00001800$

$-0.00001500$

$-0.00000400$

0.00001300

0.00001000

$-0.00000100$

$-0.87487300$

0.87491100

$-0.00000200$

0.00000600

$-0.00000700$

0.00000000

$-0.00001400$

0.00000000

Aminyl radical derived from truncated CuATSM<smiles>CNC1=NN2C=CN3N=CS[C@@]32S1</smiles>

\section{MN15/ def2TZVP Enthalpy= \\ MN15/ def2TZVP Free Energy=}

$-2904.312602$ $-2904.368053$
$\mathrm{O}^{3}$
$\mathrm{~S}$
$\mathrm{C}$
$\mathrm{N}$
$\mathrm{N}$
$\mathrm{C}$
$\mathrm{N}$
$\mathrm{N}$
$\mathrm{C}$
$\mathrm{S}$
$\mathrm{C}$
$\mathrm{H}$
$\mathrm{H}$
$\mathrm{H}$
$\mathrm{C}$
$\mathrm{N}$
$\mathrm{Cu}$
$\mathrm{H}$
$\mathrm{H}$
$\mathrm{H}$
$-1.65288800$

$-0.37459700$

0.99715400

1.13834700

2.38742000

1.19238800

1.09633800

$-0.17349700$

$-1.54080600$

0.32826100

1.36024900

0.15928400

0.15929200

2.38379800

$-0.66593600$

$-0.36028700$

$-0.30706300$

3.29271700

3.26658800
$-0.00002700$

$-0.00002400$

$-0.00001800$

$-0.00001100$

$-0.00000400$

0.00000000

0.00001100

0.00002100

0.00002400

0.00003800

0.00002300

$-0.87387800$

0.87399400

$-0.00001100$

0.00001100

$-0.00000600$

0.00003300

0.00000000

$-0.00001500$

Aminyl radical derived from truncated CuATSM 


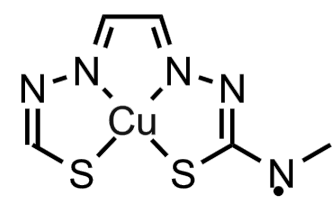

MN15-L/CBSB7 Enthalpy=

MN15-L/CBSB7 Free Energy=
$-2904.182438$

$-2904.238168$

$\mathrm{O}^{3}$
$\mathrm{~S}$
$\mathrm{C}$
$\mathrm{N}$
$\mathrm{N}$
$\mathrm{C}$
$\mathrm{N}$
$\mathrm{N}$
$\mathrm{C}$
$\mathrm{S}$
$\mathrm{C}$
$\mathrm{H}$
$\mathrm{H}$
$\mathrm{H}$
$\mathrm{C}$
$\mathrm{N}$
$\mathrm{Cu}$
$\mathrm{H}$
$\mathrm{H}$
$\mathrm{H}$

$$
\begin{array}{r}
-1.20064800 \\
-2.44042400 \\
-2.04339700 \\
-0.77286000 \\
1.20208500 \\
1.78429600 \\
3.11859100 \\
3.50031700 \\
2.48081000 \\
-4.73751800 \\
-4.40414800 \\
-5.37938800 \\
-5.37934200 \\
-0.19883700 \\
-3.67863400 \\
0.56172200 \\
4.58683400 \\
1.81796500 \\
-0.82416500
\end{array}
$$

\section{Aminyl radical derived from truncated CuATSM}<smiles>CNC1=NN2C=CN3N=CS[C@@]32S1</smiles>

\section{MN15-L/CBSB7+ Enthalpy= \\ MN15-L/CBSB7+ Free Energy=}$$
-1.64080200
$$$$
-0.34918500
$$

1.02169800

1.14869300

2.38793500

1.17328700

1.08147900

$-0.19900700$

$-1.56907600$

0.30165800

1.35575200

0.11886100

0.11887800

2.39677600

$-0.68955100$

$-0.33958600$

$-0.35937200$

3.29728600

3.29634900

\section{$-2904.232744$}

$-2904.289156$

$$
\begin{array}{r}
-0.00004700 \\
-0.00001000 \\
-0.00001200 \\
-0.00000600 \\
-0.00000300 \\
-0.00000300 \\
0.00001000 \\
0.00002600 \\
0.00003400 \\
0.00004100 \\
0.00002200 \\
-0.88345100 \\
0.88357100 \\
-0.00001000 \\
0.00002200 \\
-0.00001100 \\
0.00003700 \\
0.00000400 \\
-0.00001500
\end{array}
$$

$\mathrm{O}^{3}$
$\mathrm{~S}$
$\mathrm{C}$
$\mathrm{N}$
$\mathrm{N}$
$\mathrm{C}$
$\mathrm{N}$
$\mathrm{N}$
$\mathrm{C}$
$\mathrm{S}$
$\mathrm{C}$
$\mathrm{H}$
$\mathrm{H}$
$\mathrm{H}$
$\mathrm{C}$
$\mathrm{N}$
$\mathrm{Cu}$
$\mathrm{H}$
$\mathrm{H}$
$\mathrm{H}$

Aminyl radical derived from truncated CuATSM 
<smiles>CNC1=NN2CCN3N=CS[C@@]32S1</smiles>

MN15-L/def2TZVP Enthalpy=
MN15-L/def2TZVP Free Energy=

$\mathrm{O}^{3}$
$\mathrm{~S}$
$\mathrm{C}$
$\mathrm{N}$
$\mathrm{N}$
$\mathrm{C}$
$\mathrm{N}$
$\mathrm{N}$
$\mathrm{C}$
$\mathrm{S}$
$\mathrm{C}$
$\mathrm{H}$
$\mathrm{H}$
$\mathrm{H}$
$\mathrm{C}$
$\mathrm{N}$
$\mathrm{Cu}$
$\mathrm{H}$
$\mathrm{H}$
$\mathrm{H}$
$-2904.284944$ $-2904.340950$

$$
\begin{array}{r}
-0.00002400 \\
0.00000000 \\
-0.00000100 \\
0.00000000 \\
-0.00000900 \\
-0.00001400 \\
-0.00000500 \\
0.00001100 \\
0.00003100 \\
0.00002800 \\
0.00002200 \\
-0.88195700 \\
0.88203200 \\
-0.00000700 \\
0.00001400 \\
-0.00001100 \\
0.00002300 \\
-0.00000400 \\
-0.00000900
\end{array}
$$

Truncated analogue of CuATSM<smiles></smiles>

CBS-QB3 Enthalpy = -3092.022177 CBS-QB3 Free Energy $=-3092.088617$ B3LYP/CBSB7 Enthalpy $=-3095.006317$ B3LYP/CBSB7 Free Energy $=-3095.072493$

02

$\mathrm{Cu}$
S
C
N
N
C
N
N
C
S
N
C
H
H
H
C
O
O
C
H
H
H

$\begin{array}{rr}-1.04407700 & -0.54636200 \\ 0.18846500 & -2.37641900 \\ 1.70495300 & -1.71219900 \\ 1.74148900 & -0.32423200 \\ 0.68062100 & 0.27897500 \\ -0.83035200 & 2.08061800 \\ -1.69766800 & 1.23803700 \\ -3.02936100 & 1.52985400 \\ -3.75240400 & 0.53724000 \\ -3.24057700 & -0.99224100 \\ 2.75985700 & -2.41395600 \\ 4.01422400 & -1.91029600 \\ 3.99943600 & -0.86611000 \\ 4.77751400 & -2.04482100 \\ 4.31256800 & -2.55005400 \\ 0.62562200 & 1.71706300 \\ 1.09494700 & 2.51583300 \\ 2.55708500 & 2.54139400 \\ 3.03538400 & 2.51706400 \\ 2.75771500 & 1.58915800 \\ 4.12030200 & 2.57392000 \\ 2.67579200 & 3.38307300\end{array}$

$$
\begin{array}{r}
0.12717400 \\
0.49701100 \\
-0.14991300 \\
-0.59213400 \\
-0.39820200 \\
-0.92654500 \\
-0.50055900 \\
-0.56039000 \\
-0.10263900 \\
0.49908300 \\
-0.23997400 \\
-0.75933300 \\
-1.08148400 \\
0.01405000 \\
-1.59608800 \\
-0.77279300 \\
0.28520500 \\
0.18704100 \\
1.52472900 \\
2.03135300 \\
1.41432600 \\
2.08703400
\end{array}
$$




$\begin{array}{lrrr}\mathrm{H} & -1.11577800 & 3.04811300 & -1.33092200 \\ \mathrm{H} & 1.22323300 & 1.89593000 & -1.67214300 \\ \mathrm{H} & -4.82412600 & 0.71836300 & -0.11186400\end{array}$

$N$-methylimine derived from truncated peroxyl-substituted CuATSM<smiles></smiles>

wB97X/CBSB7 Enthalpy= wB97X/CBSB7 Free Energy=
$-3094.773128$

$-3094.837605$

$\mathrm{O}^{2}$
$\mathrm{Cu}$
$\mathrm{S}$
$\mathrm{C}$
$\mathrm{N}$
$\mathrm{N}$
$\mathrm{C}$
$\mathrm{N}$
$\mathrm{N}$
$\mathrm{C}$
$\mathrm{S}$
$\mathrm{N}$
$\mathrm{C}$
$\mathrm{H}$
$\mathrm{H}$
$\mathrm{H}$
$\mathrm{C}$
$\mathrm{O}$
$\mathrm{O}$
$\mathrm{C}$
$\mathrm{H}$
$\mathrm{H}$
$\mathrm{H}$
$\mathrm{H}$
$\mathrm{H}$
$\mathrm{H}$

$$
-1.19185900
$$$$
-0.50471400
$$$$
-2.44919300
$$$$
-1.82416600
$$$$
-0.38434200
$$$$
0.23983000
$$$$
2.26098800
$$$$
1.43146200
$$$$
1.89654200
$$$$
0.90806900
$$$$
-0.77838500
$$$$
-2.57201500
$$$$
-2.08755700
$$$$
-1.01107900
$$$$
-2.36889900
$$$$
-2.62514600
$$$$
1.71268900
$$$$
2.34888600
$$$$
\text { 2. } 37892800
$$$$
2.01896700
$$$$
0.99608900
$$$$
2.09182900
$$$$
\text { 2.72180200 }
$$$$
3.33662100
$$$$
1.89598200
$$$$
\text { 1. } 20399900
$$

$N$-methylimine derived from truncated peroxyl-substituted CuATSM<smiles></smiles>

M06-2X/Def2TZVP Enthalpy= M06-2X/Def2TZVP Free Energy=

$$
\begin{array}{r}
-1.07075900 \\
0.31643700 \\
1.78884700 \\
1.75249100 \\
0.67375400 \\
-0.89654100 \\
-1.73986600 \\
-3.07222400
\end{array}
$$

-0.07244300
0.06804100
-0.03759300
-0.21699500
-0.34991000
-0.28808200
-0.14355500
0.03404400
0.20828100
0.24169700
0.02935500
-0.07444800
-0.24146800
0.84040000
-0.89345500
-0.52989000
0.34525000
-0.27675300
0.72705100
1.07034600
0.22924500
1.56360900
-0.26336400
-1.56425800
0.35349800
$-3094.767204$

$-3094.833873$
0.10097600
0.33548100
-0.18509600
-0.58331600
-0.39766300
-0.77780400
-0.43837400
-0.45188400 
0.52644500

$-1.03903300$

$-2.19957000$

$-1.56847900$

$-0.52591300$

$-1.64861800$

$-2.15604000$

1.78097000

2.54524100

2.53419900

2.10229500

1.08085100

2.14390900

2. 77727600

3. 12561200

1.99625500

0.73106300
-0.05197300
0.45543900
-0.22994800
-0.65517300
-0.95868500
0.16487700
-1.47969300
-0.71178300
0.33737800
0.21340700
1.46000000
1.68179600
1.33863400
2.25710100
-1.06966100
-1.63899700
-0.04541900

\section{$N$-methylimine derived from truncated peroxyl-substituted CuATSM}<smiles></smiles>

M06-2X/CBSB7+ Enthalpy= M06-2X/CBSB7+ Free Energy=
$-3094.637515$

$-3094.703720$

$O^{2}$
$\mathrm{Cu}$
$\mathrm{S}$
$\mathrm{C}$
$\mathrm{N}$
$\mathrm{N}$
$\mathrm{C}$
$\mathrm{N}$
$\mathrm{N}$
$\mathrm{C}$
$\mathrm{S}$
$\mathrm{N}$
$\mathrm{C}$
$\mathrm{H}$
$\mathrm{H}$
$\mathrm{H}$
$\mathrm{C}$
$\mathrm{O}$
$\mathrm{O}$
$\mathrm{C}$
$\mathrm{H}$
$\mathrm{H}$
$\mathrm{H}$
$\mathrm{H}$
$\mathrm{H}$
$\mathrm{H}$

$$
\begin{array}{r}
-1.08925900 \\
0.27012300 \\
1.76447900 \\
1.74568600 \\
0.66359200 \\
-0.87993500 \\
-1.73333000 \\
-3.06254200 \\
-3.79846400 \\
-3.34274900 \\
2.88084300 \\
4.12189000 \\
4.03426100 \\
4.81369000 \\
4.55462700 \\
0.58213800 \\
1.14051000 \\
2.55697800 \\
3.11317800 \\
2.80982700 \\
4.19020800 \\
2.82656200 \\
-1.17694700 \\
1.10202700 \\
-4.86310300
\end{array}
$$

0.08567900

0.29285300

$-0.18156000$

$-0.57454100$

$-0.40036000$

$-0.72985000$

$-0.40402900$

$-0.39677800$

$-0.01698900$

0.44272400

$-0.19203000$

$-0.58488900$

$-0.89734000$

0.25789300

$-1.39407600$

$-0.70107100$

0.34042000

0.16289400

1. 39535500

1.63091700

1. 23291800

2. 19917700

$-0.98806600$

$-1.64067100$

0.00700800

$\mathrm{N}$-methylimine derived from truncated peroxyl-substituted CuATSM 


$$
\begin{aligned}
& \text { OOMe }
\end{aligned}
$$

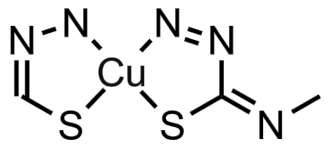

M06-2X-D3/CBSB7 Enthalpy= M06-2X-D3/CBSB7 Free Energy=

02
$C u$
$C$
C
$C$
N
N
C
N
N
C
S
N
C
H
H
H
C
O
O
C
H
H
H
H
H
H

\section{$-3094.595535$ \\ $-3094.660628$}

$$
\begin{array}{rr}
-0.60200800 & 0.09345600 \\
-2.37802500 & 0.38399100 \\
-1.58430700 & -0.19221100 \\
-0.17708200 & -0.60890900 \\
0.35234300 & -0.41794200 \\
2.11638600 & -0.81536700 \\
1.21522000 & -0.45023500 \\
1.48540900 & -0.46868400 \\
0.46196300 & -0.05804400 \\
-1.09075000 & 0.46065300 \\
-2.17291600 & -0.24864600 \\
-1.53786500 & -0.71720700 \\
-0.49816300 & -1.02974100 \\
-1.60777900 & 0.08421800 \\
-2.13491800 & -1.54682600 \\
1.79538300 & -0.72898800 \\
2.55232000 & 0.34447200 \\
2.53402100 & 0.26734000 \\
2.07136700 & 1.52394500 \\
1.04423100 & 1.71083700 \\
2.11463400 & 1.44051800 \\
2.73011900 & 2.32426400 \\
3.10315700 & -1.12680400 \\
2.02715100 & -1.63939000 \\
0.63110700 & -0.04795300 \\
&
\end{array}
$$

$\mathrm{N}$-methylimine derived from truncated peroxyl-substituted CuATSM<smiles></smiles>

M06-2X/CBSB7 Enthalpy=
M06-2X/CBSB7 Free Energy=

$\mathrm{O}^{2}$
$\mathrm{Cu}$
$\mathrm{S}$
$\mathrm{C}$
$\mathrm{N}$
$\mathrm{N}$
$\mathrm{C}$
$\mathrm{N}$
$\mathrm{N}$
$\mathrm{C}$
$\mathrm{S}$
$\mathrm{N}$
$\mathrm{C}$
$\mathrm{H}$
$\mathrm{H}$
$-3094.594042$ $-3094.659163$

$$
\begin{array}{r}
-0.60122300 \\
-2.38104400 \\
-1.58745900 \\
-0.17963000 \\
0.35098500 \\
2.12064200 \\
1.21986800 \\
1.49402900 \\
0.47041800 \\
-1.08641800 \\
-2.17662600 \\
-1.54155400 \\
-0.50119800 \\
-1.61345900
\end{array}
$$




$\begin{array}{lrrr}\mathrm{H} & 4.51874000 & -2.13741700 & -1.53495400 \\ \mathrm{C} & 0.55095600 & 1.79495000 & -0.72543700 \\ \mathrm{O} & 1.03810300 & 2.54954500 & 0.34597600 \\ \mathrm{O} & 2.46258700 & 2.53037800 & 0.25880600 \\ \mathrm{C} & 2.93648100 & 2.06386900 & 1.51059800 \\ \mathrm{H} & 2.61583400 & 1.03630400 & 1.69683900 \\ \mathrm{H} & 4.02173200 & 2.10686200 & 1.41912400 \\ \mathrm{H} & 2.60196900 & 2.72057100 & 2.31533200 \\ \mathrm{H} & -1.25152600 & 3.10999600 & -1.11107300 \\ \mathrm{H} & 1.10765100 & 2.02573500 & -1.63837000 \\ \mathrm{H} & -4.85620200 & 0.64250400 & -0.03998300\end{array}$

$\mathrm{N}$-methylimine derived from truncated peroxyl-substituted CuATSM<smiles></smiles>

M06/CBSB7 Enthalpy=
M06/CBSB7 Free Energy=

02

$\mathrm{Cu}$

S

C

$\mathrm{N}$

$\mathrm{N}$

C

N

$\mathrm{N}$

C

S

$\mathrm{N}$

C

$\mathrm{H}$

$\mathrm{H}$

$\mathrm{H}$

C

o

O

C

$\mathrm{H}$

$\mathrm{H}$

$\mathrm{H}$

$\mathrm{H}$

$\mathrm{H}$

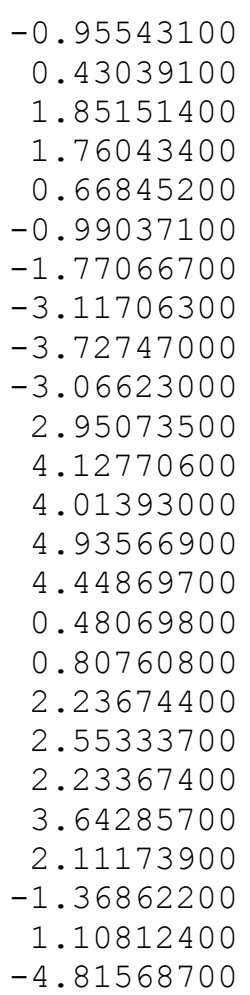

$-3094.410652$

$-3094.476151$
0.12558900
0.54262200
-0.20261100
-0.68418800
-0.44941000
-0.99574100
-0.54348300
-0.62298000
-0.13939900
0.51003700
-0.31200200
-0.90150100
-1.26555500
-0.16188400
-1.72639500
-0.79434300
0.32210400
0.41716500
1.72448600
1.90249500
1.77298700
2.47512100
-1.44297400
-1.65440000
-0.15907500

$\mathrm{N}$-methylimine derived from truncated peroxyl-substituted CuATSM<smiles></smiles>

M06/CBSB7+ Enthalpy=

$-3094.461900$

M06/CBSB7+ Free Energy=

$-3094.527577$

02

$\mathrm{Cu}$

$-1.05505400$

$-0.57592400$

0.09041000 
$-2.39195800$

$-1.61874500$

$-0.22695900$

0.33286300

2.13552300

1.24912200

1.53146400

0.50769200

$-1.04644000$

$-2.23480300$

$-1.64892100$

$-0.62135200$

$-1.67863300$

$-2.28894300$

1.77653100

2.52351600

2.50420100

2.11933500

1.10301400

2.15305100

2.82515700

3.13908400

1.99224700

0.68050500
0.29650000

$-0.19920200$

$-0.63601000$

$-0.41615300$

$-0.78472000$

$-0.42687400$

$-0.42600700$

$-0.01247300$

0.48335000

$-0.17929800$

$-0.58647400$

$-0.96122200$

0.26563700

$-1.35523700$

$-0.72937200$

0.32737200

0.19383100

1.44997300

1.71979500

1. 31735900

2. 23230700

$-1.08171400$

$-1.65897800$

0.01693200

\section{$N$-methylimine derived from truncated peroxyl-substituted CuATSM}<smiles></smiles>

M06/Def2TZVP Enthalpy= M06/Def2TZVP Free Energy=

\section{$-3094.618548$}

$-3094.684150$

$\begin{array}{ll}0 & 2 \\ \mathrm{Cu} \\ \mathrm{S} \\ \mathrm{C} \\ \mathrm{N} \\ \mathrm{N} \\ \mathrm{C} \\ \mathrm{N} \\ \mathrm{N} \\ \mathrm{C} \\ \mathrm{S} \\ \mathrm{N} \\ \mathrm{C} \\ \mathrm{H} \\ \mathrm{H} \\ \mathrm{H} \\ \mathrm{C} \\ \mathrm{O} \\ \mathrm{O} \\ \mathrm{C} \\ \mathrm{H} \\ \mathrm{H} \\ \mathrm{H} \\ \mathrm{H} \\ \mathrm{H} \\ \mathrm{H}\end{array}$

$-0.56917700$

$-2.41275600$

$-1.65671300$

$-0.25345100$

0.31698200

2.16445400

1.29004600

1.60693000

0.58951800

$-0.99598400$

$-2.29118100$

$-1.72333300$

$-0.68059100$

$-1.80675800$

$-2.33838800$

1. 76562900

2.50293100

2.47974500

2.09166000

1.07388600

2.13079700

2.78836600

3.19117600

1.96046800

0.79305400
0.06523200

0.25124100

$-0.17614700$

$-0.56900300$

$-0.39053300$

$-0.69284800$

$-0.38168400$

$-0.35071200$

0.02428500

0.44832900

$-0.15140400$

$-0.49794900$

$-0.82834400$

0.36834000

$-1.28088200$

$-0.68749000$

0.33101700

0.10366400

1. 31880200

1.60368600

1. 12385600

2. 12329700

$-0.92938600$

$-1.64329300$

0.07186900 
$N$-methylimine derived from truncated peroxyl-substituted CuATSM<smiles></smiles>

M06-L/CBSB7 Enthalpy= M06-L/CBSB7 Free Energy=
$-3094.789803$

$-3094.856111$
0.10464400

0.40167800

$-0.22515400$

$-0.68625700$

$-0.40650300$

$-0.86985600$

$-0.45449300$

$-0.49556500$

$-0.05883800$

0.47875400

$-0.26976800$

$-0.74309100$

$-1.10963400$

0.07027500

$-1.53237000$

$-0.74602400$

0.35247100

0.31477100

1.57372600

1. 75234900

1.51047100

2.38844800

$-1.24639100$

$-1.64034300$

$-0.04881400$

$\mathrm{N}$-methylimine derived from truncated peroxyl-substituted CuATSM<smiles></smiles>

M06-L/CBSB7+ Enthalpy=

M06-L/CBSB7+ Free Energy=
$-3094.839584$

$-3094.905567$

$$
\begin{array}{r}
-0.59449700 \\
-2.36841400 \\
-1.56644000 \\
-0.19066400 \\
0.34669200 \\
2.12489500 \\
1.22358200 \\
1.48145500 \\
0.44608900 \\
-1.07944800 \\
-2.17609200 \\
-1.54851700 \\
-0.52576000
\end{array}
$$




$\begin{array}{lrrr}\mathrm{H} & 4.83271300 & -1.55336300 & 0.19168100 \\ \mathrm{H} & 4.58190200 & -2.17668400 & -1.41832400 \\ \mathrm{C} & 0.52930900 & 1.78754700 & -0.74508100 \\ \mathrm{O} & 1.02370700 & 2.53291000 & 0.34368600 \\ \mathrm{O} & 2.48843700 & 2.49682900 & 0.25313200 \\ \mathrm{C} & 2.94996300 & 2.08103200 & 1.52219300 \\ \mathrm{H} & 2.60989400 & 1.06990100 & 1.77039500 \\ \mathrm{H} & 4.03746200 & 2.08779000 & 1.41889100 \\ \mathrm{H} & 2.65426600 & 2.78256000 & 2.30795600 \\ \mathrm{H} & -1.24542800 & 3.11290500 & -1.15679500 \\ \mathrm{H} & 1.10108200 & 2.02340800 & -1.65290400 \\ \mathrm{H} & -4.85790500 & 0.60509400 & -0.01520400\end{array}$

$N$-methylimine derived from truncated peroxyl-substituted CuATSM<smiles></smiles>

M06-L/Def2TZVP Enthalpy= M06-L/Def2TZVP Free Energy=

0
$\mathrm{Cu}$
$\mathrm{S}$
$\mathrm{C}$
$\mathrm{N}$
$\mathrm{N}$
$\mathrm{C}$
$\mathrm{N}$
$\mathrm{N}$
$\mathrm{C}$
$\mathrm{S}$
$\mathrm{N}$
$\mathrm{C}$
$\mathrm{H}$
$\mathrm{H}$
$\mathrm{H}$
$\mathrm{C}$
$\mathrm{O}$
$\mathrm{O}$
$\mathrm{C}$
$\mathrm{H}$
$\mathrm{H}$
$\mathrm{H}$
$\mathrm{H}$
$\mathrm{H}$
$\mathrm{H}$

-1.04267100
0.31356300
1.78325000
1.73335000
0.64587900
-0.92139400
-1.75990900
-3.09179900
-3.77392500
-3.23122800
2.89910700
4.11750900
4.02217800
4.82728900
4.56043200
0.53154300
1.02550300
2.48877700
2.94728500
2.60022600
4.03216500
2.66065100
-1.23554300
1.09558400
-4.84913400

$-0.59246100$

$-2.36362400$

$-1.56957700$

$-0.19172100$

0.34750400

2.11970100

1.22390300

1.47610200

0.44459300

$-1.07722000$

$-2.17970900$

$-1.56396500$

$-0.53787900$

$-1.58495000$

$-2.18550800$

1.78557100

2.53859400

2.49952300

2.08708700

1.08325200

2.08164900

2.79202600

3.10754100

2.01503800

0.60576000
0.10393900

0.32987000

$-0.21012000$

$-0.64886700$

$-0.41512700$

$-0.82419300$

$-0.45451700$

$-0.47445900$

$-0.04073700$

0.49381200

$-0.22549900$

$-0.65785100$

$-1.02030400$

0.17257500

$-1.43856100$

$-0.73738900$

0.33976000

0.24782100

1.51478500

1.77151800

1.41350900

2.29753900

$-1.15018700$

$-1.64897500$

$-0.02857700$

$\mathrm{N}$-methylimine derived from truncated peroxyl-substituted CuATSM<smiles></smiles>

MN15/CBSB7 Enthalpy=

$-3094.377692$

MN15/CBSB7 Free Energy=

$-3094.442811$

02 


$\begin{array}{lrrr}\mathrm{Cu} & -0.95822100 & -0.56801200 & 0.11949300 \\ \mathrm{~S} & 0.43829000 & -2.22652400 & 0.52735000 \\ \mathrm{C} & 1.85405200 & -1.48307200 & -0.20096100 \\ \mathrm{~N} & 1.76206000 & -0.10788000 & -0.66659300 \\ \mathrm{~N} & 0.65860900 & 0.39496900 & -0.44309400 \\ \mathrm{C} & -1.00577000 & 2.03289900 & -0.97297400 \\ \mathrm{~N} & -1.78017300 & 1.11134100 & -0.54072300 \\ \mathrm{~N} & -3.13428700 & 1.25971200 & -0.61183500 \\ \mathrm{C} & -3.73848400 & 0.20534600 & -0.13203200 \\ \mathrm{~S} & -3.05373900 & -1.22409300 & 0.50280900 \\ \mathrm{~N} & 2.96032600 & -2.08998900 & -0.31831400 \\ \mathrm{C} & 4.13722400 & -1.49525900 & -0.90684600 \\ \mathrm{H} & 4.01367300 & -0.46398000 & -1.24522800 \\ \mathrm{H} & 4.94617400 & -1.54522800 & -0.17309400 \\ \mathrm{H} & 4.44895000 & -2.12153400 & -1.74697100 \\ \mathrm{C} & 0.47258000 & 1.81732400 & -0.77974600 \\ \mathrm{O} & 0.81203800 & 2.59106100 & 0.33762100 \\ \mathrm{O} & 2.23920400 & 2.59202400 & 0.41936700 \\ \mathrm{C} & 2.55996900 & 2.10599100 & 1.71154100 \\ \mathrm{H} & 2.20824200 & 1.07931300 & 1.84927500 \\ \mathrm{H} & 3.64880500 & 2.13550000 & 1.75461000 \\ \mathrm{H} & 2.13912500 & 2.75390400 & 2.48301300 \\ \mathrm{H} & -1.39361200 & 2.95687400 & -1.39248600 \\ \mathrm{H} & 1.09537200 & 2.08925900 & -1.63759800 \\ \mathrm{H} & -4.82425800 & 0.25702500 & -0.14523300\end{array}$

$\mathrm{N}$-methylimine derived from truncated peroxyl-substituted CuATSM<smiles></smiles>

$\mathrm{MN} 15 / \mathrm{CBSB} 7+$ Enthalpy= $\mathrm{MN} 15 / \mathrm{CBSB} 7+$ Free Energy=

02

$\mathrm{Cu}$
$\mathrm{S}$
$\mathrm{C}$
$\mathrm{N}$
$\mathrm{N}$
$\mathrm{C}$
$\mathrm{N}$
$\mathrm{N}$
$\mathrm{C}$
$\mathrm{S}$
$\mathrm{N}$
$\mathrm{C}$
$\mathrm{H}$
$\mathrm{H}$
$\mathrm{H}$
$\mathrm{C}$
$\mathrm{O}$
$\mathrm{O}$
$\mathrm{C}$
$\mathrm{H}$
$\mathrm{H}$
$\mathrm{H}$
$\mathrm{H}$
$\mathrm{H}$
$\mathrm{H}$

\section{$-3094.427307$ \\ $-3094.492312$}

-0.09395900
0.07758500
-0.02298600
-0.18870500
-0.35814500
-0.24434800
-0.10692700
0.08891100
0.23121300
0.20276500
0.04511200
-0.04477900
-0.18078300
0.85877100
-0.87823000
-0.51977700
0.33953500
-0.31547000
0.68516300
1.09270600
0.16974900
1.47925400
-0.19444900
-1.55888400
0.38991600 
$N$-methylimine derived from truncated peroxyl-substituted CuATSM<smiles></smiles>

$\begin{array}{ll}\text { MN15/ def2TZVP Enthalpy }= & -3094.416556 \\ \text { MN15/ def2TZVP Free Energy }= & -3094.481641\end{array}$

$\begin{array}{lrrr}02 & & \\ \mathrm{Cu} & -1.20129700 & -0.51233100 & -0.09179000 \\ \mathrm{~S} & -0.13746600 & -2.44815800 & 0.07259000 \\ \mathrm{C} & 1.48425000 & -1.80944200 & -0.02562400 \\ \mathrm{~N} & 1.68190100 & -0.37943600 & -0.19555900 \\ \mathrm{~N} & 0.63729200 & 0.24135600 & -0.35878200 \\ \mathrm{C} & -0.65647800 & 2.26014800 & -0.24941700 \\ \mathrm{~N} & -1.62001000 & 1.43860600 & -0.10787100 \\ \mathrm{~N} & -2.89107400 & 1.89247900 & 0.08668300 \\ \mathrm{C} & -3.72280400 & 0.89925000 & 0.23153400 \\ \mathrm{~S} & -3.41335800 & -0.77239100 & 0.20684200 \\ \mathrm{~N} & 2.50782400 & -2.55097500 & 0.04805300 \\ \mathrm{C} & 3.86067900 & -2.06788500 & -0.03994100 \\ \mathrm{H} & 3.95670700 & -0.98986700 & -0.18475400 \\ \mathrm{H} & 4.38943200 & -2.36583700 & 0.86779900 \\ \mathrm{H} & 4.35318700 & -2.58534100 & -0.86561700 \\ \mathrm{C} & 0.71818900 & 1.71064100 & -0.52070700 \\ \mathrm{O} & 1.61128500 & 2.32989600 & 0.33691200 \\ \mathrm{O} & 2.88319800 & 2.33729400 & -0.31397100 \\ \mathrm{C} & 3.82963600 & 2.03533600 & 0.68753100 \\ \mathrm{H} & 3.64547600 & 1.04429900 & 1.10541900 \\ \mathrm{H} & 4.79050700 & 2.05633500 & 0.17548300 \\ \mathrm{H} & 3.81401400 & 2.79043600 & 1.47512100 \\ \mathrm{H} & -0.80610500 & 3.33488600 & -0.20429900 \\ \mathrm{H} & 1.01459300 & 1.90319500 & -1.55926000 \\ \mathrm{H} & -4.75523800 & 1.19826900 & 0.38966000\end{array}$

$\mathrm{N}$-methylimine derived from truncated peroxyl-substituted CuATSM<smiles></smiles>

MN15-L/CBSB7 Enthalpy= MN15-L/CBSB7 Free Energy=
$-3094.273018$ $-3094.337359$

\footnotetext{
02
}

$\begin{array}{lr}\mathrm{Cu} & -0.87136800 \\ \mathrm{~S} & 0.69180900 \\ \mathrm{C} & 2.01723300 \\ \mathrm{~N} & 1.81204300 \\ \mathrm{~N} & 0.64091300 \\ \mathrm{C} & -1.14051000 \\ \mathrm{~N} & -1.85098500 \\ \mathrm{~N} & -3.21271000 \\ \mathrm{C} & -3.72904000 \\ \mathrm{~S} & -2.92288300 \\ \mathrm{~N} & 3.16286600 \\ \mathrm{C} & 4.27098300\end{array}$

0.12342100
0.61734400
-0.23112600
-0.74361000
-0.53740300
-1.13137600
-0.59511300
-0.71778200
-0.19004500
0.51956100
-0.36132600
-1.04248300 


$\begin{array}{lrrr}\mathrm{H} & 4.07855800 & -0.19266900 & -1.42247500 \\ \mathrm{H} & 5.13322600 & -1.19387800 & -0.34848300 \\ \mathrm{H} & 4.57572000 & -1.86706500 & -1.88196800 \\ \mathrm{C} & 0.34095400 & 1.86699700 & -0.83231800 \\ \mathrm{O} & 0.49026700 & 2.57422800 & 0.38280200 \\ \mathrm{O} & 1.91613100 & 2.62938800 & 0.65740100 \\ \mathrm{C} & 2.07751500 & 2.01891300 & 1.93036800 \\ \mathrm{H} & 1.75182700 & 0.95863200 & 1.92166400 \\ \mathrm{H} & 3.16350900 & 2.07825400 & 2.12050700 \\ \mathrm{H} & 1.52813300 & 2.57710300 & 2.71151900 \\ \mathrm{H} & -1.59226800 & 2.77868800 & -1.68446100 \\ \mathrm{H} & 1.01719600 & 2.26957700 & -1.61239300 \\ \mathrm{H} & -4.82792200 & -0.12082400 & -0.22668600\end{array}$

$\mathrm{N}$-methylimine derived from truncated peroxyl-substituted CuATSM<smiles></smiles>

MN15-L/CBSB7+ Enthalpy= MN15-L/CBSB7+ Free Energy=
$-3094.326659$ $-3094.391315$
0.13842100
0.58468300
-0.23718800
-0.74851600
-0.55648400
-1.11841100
-0.60470300
-0.71775600
-0.19331100
0.51710100
-0.35093800
-1.02560500
-1.42068100
-0.32143700
-1.85334500
-0.83653700
0.38386000
0.65946500
1.93989900
1.93617900
2.13232100
2.71172900
-1.64514500
-1.61910900
-0.23418700

$-0.88317900$

0.71922300

2.03529400

1.82052000

0.64524000

$-1.13814100$

$-1.85672600$

$-3.21759900$

$-3.75307200$

$-2.98194100$

3.19398800

4.30134700

4.09765800

5.15448400

4.62483800

0.34614600

0.50477600

1.93095900

2.09086600

1.76589900

3.17628500

1.53605400

$-1.58146100$

1.01659900

$-4.85317800$

$\mathrm{N}$-methylimine derived from truncated peroxyl-substituted CuATSM<smiles></smiles>

MN15-L/def2TZVP Enthalpy=

$-3094.397226$

MN15-L/def2TZVP Free Energy=

$-3094.462009$ 


$\begin{array}{lrrr}02 & & & \\ \mathrm{Cu} & -0.89085900 & -0.60239800 & 0.14120400 \\ \mathrm{~S} & 0.68940300 & -2.13556300 & 0.57730400 \\ \mathrm{C} & 2.01139900 & -1.30413600 & -0.23317600 \\ \mathrm{~N} & 1.81048700 & 0.04474800 & -0.73397800 \\ \mathrm{~N} & 0.64942900 & 0.44270300 & -0.52450300 \\ \mathrm{C} & -1.12174100 & 1.96574800 & -1.09513000 \\ \mathrm{~N} & -1.84642700 & 1.01873600 & -0.59941200 \\ \mathrm{~N} & -3.20538800 & 1.06547600 & -0.71375000 \\ \mathrm{C} & -3.74474800 & -0.02235800 & -0.19429800 \\ \mathrm{~S} & -2.98795600 & -1.37896400 & 0.51156200 \\ \mathrm{~N} & 3.16003100 & -1.85938100 & -0.36046600 \\ \mathrm{C} & 4.27228300 & -1.21340200 & -1.02126900 \\ \mathrm{H} & 4.07983300 & -0.19396500 & -1.39653300 \\ \mathrm{H} & 5.12380100 & -1.19368800 & -0.31767100 \\ \mathrm{H} & 4.59022800 & -1.85927600 & -1.85961400 \\ \mathrm{C} & 0.36216500 & 1.86324100 & -0.82154700 \\ \mathrm{O} & 0.54350700 & 2.58263500 & 0.37871500 \\ \mathrm{O} & 1.97416200 & 2.60827300 & 0.63304700 \\ \mathrm{C} & 2.13259100 & 2.01054200 & 1.90958600 \\ \mathrm{H} & 1.77542900 & 0.96219600 & 1.91727700 \\ \mathrm{H} & 3.21965900 & 2.04072700 & 2.08972200 \\ \mathrm{H} & 1.60735800 & 2.59197800 & 2.68759300 \\ \mathrm{H} & -1.55758600 & 2.83039900 & -1.61258300 \\ \mathrm{H} & 1.02602500 & 2.24695700 & -1.62034000 \\ \mathrm{H} & -4.84295500 & -0.03441100 & -0.23895200 \\ & & & \end{array}$

\section{Methyl peroxide}

\begin{tabular}{|c|c|c|c|}
\hline \multirow{2}{*}{\multicolumn{2}{|c|}{$\begin{array}{l}\text { CBS-QB3 Enthalpy }=-190.589542 \\
\text { B3LYP/CBSB7 Enthalpy }=-190.850939\end{array}$}} & \multicolumn{2}{|c|}{ CBS-QB3 Free Energy $=-1$} \\
\hline & & \multicolumn{2}{|c|}{ B3LYP/CBSB7 Free Energ } \\
\hline & & & \\
\hline $\mathrm{C}$ & -1.12961700 & -0.22363900 & 0.02672600 \\
\hline 0 & 0.01619300 & 0.60684200 & -0.03138300 \\
\hline 0 & 1.16412700 & -0.28550800 & -0.09072600 \\
\hline $\mathrm{H}$ & -1.97292100 & 0.47126800 & 0.02466200 \\
\hline $\mathrm{H}$ & -1.18954500 & -0.87767400 & -0.84877700 \\
\hline $\mathrm{H}$ & -1.14400500 & -0.82582100 & 0.94203100 \\
\hline $\mathrm{H}$ & 1.64161800 & 0.00339000 & 0.6985980 \\
\hline
\end{tabular}

Methyl peroxide

\section{wB97X/CBSB7 Enthalpy= wB97X/CBSB7 Free Energy= \\ $-190.794046$ \\ $-190.824706$} 01

$\begin{array}{lr}\mathrm{C} & -1.12035300 \\ \mathrm{O} & 0.02749400 \\ \mathrm{O} & 1.14735700 \\ \mathrm{H} & -1.96279900 \\ \mathrm{H} & -1.17784400 \\ \mathrm{H} & -1.13434500 \\ \mathrm{H} & 1.59829400\end{array}$

$$
\begin{array}{r}
-0.21905300 \\
0.60039400 \\
-0.27517900 \\
0.47557500 \\
-0.86319800 \\
-0.83294000 \\
-0.06683500
\end{array}
$$

\section{Methyl peroxide}

M06-2X/Def2TZVP Enthalpy=
M06-2X/Def2TZVP Free Energy=
01
$\begin{array}{lr}\mathrm{C} & -1.11707500 \\ 0 & 0.02634800\end{array}$

\author{
$-190.789594$ \\ $-190.820128$ \\ $-0.22075900$ \\ 0.60072000
}




$\begin{array}{rrrr}\mathrm{O} & 1.14650900 & -0.26745200 & -0.10250700 \\ \mathrm{H} & -1.96129900 & 0.46799100 & 0.03310900 \\ \mathrm{H} & -1.16688600 & -0.85554700 & -0.86516200 \\ \mathrm{H} & -1.12760700 & -0.83957000 & 0.92175200 \\ \mathrm{H} & 1.57538300 & -0.11445800 & 0.74921000\end{array}$

Methyl peroxide

$\begin{array}{ll}\text { M06-2X/CBSB7+ Enthalpy }= & -190.768744 \\ \text { M06-2X/CBSB7+ Free Energy }= & -190.799504\end{array}$

01

C -1.12378600

O $\quad 0.02692100$

$0 \quad 1.13840500$

$\mathrm{H} \quad-1.96133600$

$\mathrm{H} \quad-1.17419800$

$\mathrm{H} \quad-1.13874700$

$\mathrm{H} \quad 1.69439000$

Methyl peroxide

\section{M06-2X-D3/CBSB7 Enthalpy= M06-2X-D3/CBSB7 Free Energy=}

01

C -1.11939100

o 0.02642500

O 1.14405500

$\mathrm{H} \quad-1.96265100$

$\mathrm{H} \quad-1.17036900$

$\mathrm{H} \quad-1.12934400$

$\mathrm{H} \quad 1.61487800$

$$
\begin{array}{r}
-0.21494600 \\
0.59755300 \\
-0.29498100 \\
0.48295100 \\
-0.87927500 \\
-0.80048300 \\
0.06591400
\end{array}
$$

0.02903500
-0.03565800
-0.07955100
0.01144900
-0.83686900
0.95206400
0.62082000

Methyl peroxide

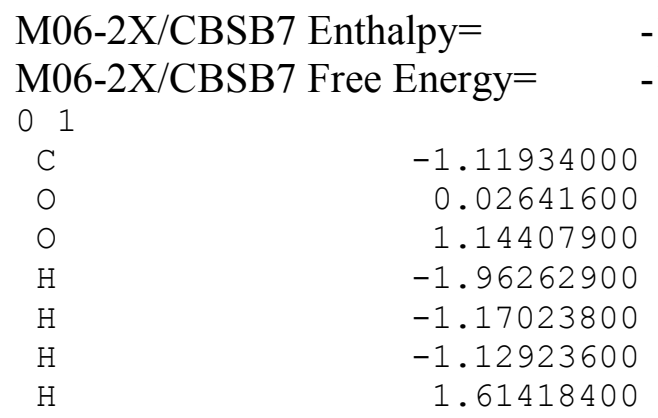

$-190.762245$

$-190.792829$

$$
\begin{array}{r}
-0.21915800 \\
0.60086800 \\
-0.27857100 \\
0.47220900 \\
-0.86746300 \\
-0.82519000 \\
-0.04299100
\end{array}
$$

0.02505200

$-0.02673300$

$-0.09472100$

0.02593100

$-0.85272700$

0.93570100

0.71240900

\section{Methyl peroxide}

\section{M06/CBSB7 Enthalpy= M06/CBSB7 Free Energy=}

$$
\begin{array}{r}
-1.11500500 \\
0.02336700 \\
1.14719800 \\
-1.96274700 \\
-1.17642100 \\
-1.13431700 \\
1.59899200
\end{array}
$$

$-190.736736$

$-190.767310$

$$
\begin{array}{r}
-0.21894700 \\
0.60075500 \\
-0.27528800 \\
0.47133400 \\
-0.86918800 \\
-0.83364300 \\
-0.05855000
\end{array}
$$


Methyl peroxide

$\begin{array}{lrrr}\text { M06/CBSB7+ Enthalpy= } & -190.743904 & \\ \text { M06/CBSB7+ Free Energy= } & -190.774592 & \\ \text { O 1 } & -1.12049700 & -0.21401800 & 0.02928500 \\ \text { C } & 0.02368500 & 0.59708300 & -0.03648300 \\ \text { O } & 1.14036300 & -0.29597800 & -0.07841600 \\ \text { O } & -1.96158100 & 0.48369900 & 0.01258200 \\ \text { H } & -1.18200600 & -0.88234700 & -0.83751700 \\ \text { H } & -1.14365100 & -0.80481100 & 0.95337600 \\ \text { H } & 1.69784000 & 0.07872500 & 0.61503800 \\ \text { H } & & & \end{array}$

Methyl peroxide

$\begin{array}{lrrr}\text { M06/Def2TZVP Enthalpy= } & -190.766314 & \\ \text { M06/Def2TZVP Free Energy }= & -190.796922 & \\ \text { O } 1 & & & \\ \text { C } & -1.11218500 & -0.21948700 & 0.02137100 \\ \text { O } & 0.02365200 & 0.60066100 & -0.01902700 \\ \text { O } & 1.14710800 & -0.26805500 & -0.10210300 \\ \text { H } & -1.96157900 & 0.46582300 & 0.03331400 \\ \text { H } & -1.17339100 & -0.85980100 & -0.86400000 \\ \text { H } & -1.13217800 & -0.84314100 & 0.92246100 \\ \text { H } & 1.57417700 & -0.10680700 & 0.74904300\end{array}$

Methyl peroxide

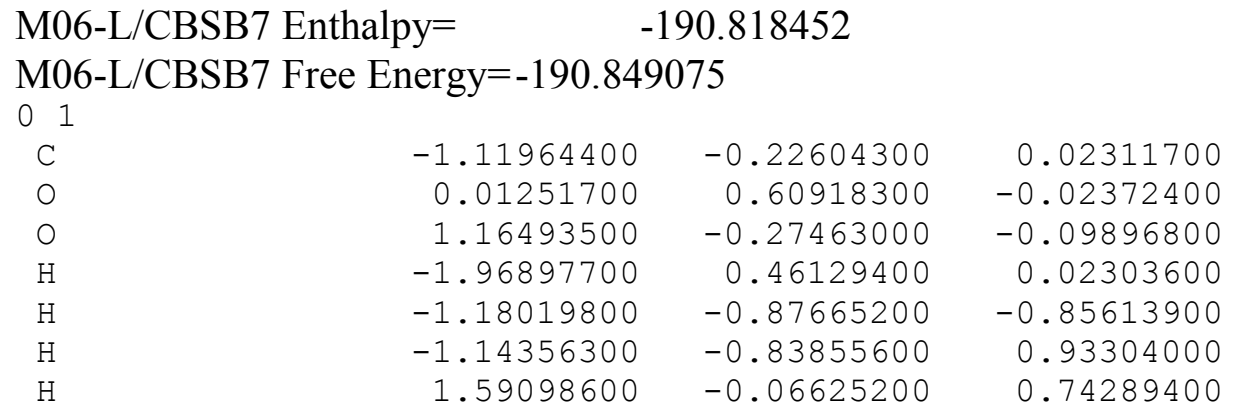

Methyl peroxide

$\begin{array}{lrrr}\text { M06-L/CBSB7+ Enthalpy= } & -190.824243 & \\ \text { M06-L/CBSB7+ Free Energy }= & -190.855040 & \\ \text { O } 1 & & & \\ \text { C } & -1.12496200 & -0.22103300 & 0.02945000 \\ \text { O } & 0.01190300 & 0.60555000 & -0.03731400 \\ \text { O } & 1.15785600 & -0.29759300 & -0.07889200 \\ \text { H } & -1.96783500 & 0.47345200 & 0.00958700 \\ \text { H } & -1.18668200 & -0.89083300 & -0.83501600 \\ \text { H } & -1.15089900 & -0.80883300 & 0.95421500 \\ \text { H } & 1.69711900 & 0.08875700 & 0.62416100\end{array}$

Methyl peroxide

M06-L/Def2TZVP Enthalpy= M06-L/Def2TZVP Free Energy=

$-190.841090$ $-190.871699$ 01 
Methyl peroxide

\section{MN15/CBSB7 Enthalpy= MN15/CBSB7 Free Energy= \\ $-190.639941$ \\ $-190.670733$} 01

$\begin{array}{lr}\mathrm{C} & -1.11753800 \\ \mathrm{O} & 0.02557800 \\ \mathrm{O} & 1.14203400 \\ \mathrm{H} & -1.96648200 \\ \mathrm{H} & -1.17087700 \\ \mathrm{H} & -1.12741400 \\ \mathrm{H} & 1.62910300\end{array}$

Methyl peroxide

$\mathrm{MN} 1$
$\mathrm{MN} 1$
$\mathrm{O} 1$
$\mathrm{C}$
$\mathrm{O}$
$\mathrm{O}$
$\mathrm{H}$
$\mathrm{H}$
$\mathrm{H}$
$\mathrm{H}$

Methyl peroxide

\section{MN15/ def2TZVP Enthalpy=} MN15/ def2TZVP Free Energy= 01

$\begin{array}{lr}\mathrm{C} & -1.11496700 \\ \mathrm{O} & 0.02606500 \\ \mathrm{O} & 1.14185200 \\ \mathrm{H} & -1.96346100 \\ \mathrm{H} & -1.16756100 \\ \mathrm{H} & -1.12431400 \\ \mathrm{H} & 1.60180100\end{array}$

$$
\begin{array}{r}
-0.21834000 \\
0.60278400 \\
-0.28203400 \\
0.46663200 \\
-0.87061900 \\
-0.82551900 \\
-0.02645100
\end{array}
$$

0.02591700
-0.02832500
-0.09306600
0.02680000
-0.84999300
0.93686300
0.70194900
$-190.646952$

$-190.677789$

$$
\begin{array}{rr}
-0.21422500 & 0.02924800 \\
0.59930300 & -0.03623700 \\
-0.29790200 & -0.07753000 \\
0.47808400 & 0.01553700 \\
-0.87872100 & -0.83716000 \\
-0.80402200 & 0.95051800 \\
0.07880800 & 0.60575500
\end{array}
$$

\section{Methyl peroxide}

$\begin{array}{lrrr}\text { MN15-L/CBSB7 Enthalpy= } & -190.656310 & \\ \begin{array}{llrr}\text { MN15-L/CBSB7 Free Energy= } & -190.686814 & \\ \text { O }^{1} & -1.11412900 & -0.22774500 & 0.01697300 \\ \text { C } & 0.01885200 & 0.61404000 & -0.01142000 \\ \text { O } & 1.15921000 & -0.26285600 & -0.10835400 \\ \text { O } & -1.97893600 & 0.46006400 & 0.03162100 \\ \text { H } & -1.16283500 & -0.87003600 & -0.88412700 \\ \text { H } & -1.12851800 & -0.86588900 & 0.92551400 \\ \text { H } & 1.53056400 & -0.16714900 & 0.78335000 \\ \text { H } & & & \end{array}\end{array}$

$-190.671868$

$-190.702537$

$$
\begin{array}{r}
-0.21922800 \\
0.60168300 \\
-0.27357400 \\
0.46429000 \\
-0.86105600 \\
-0.83463100 \\
-0.07810300
\end{array}
$$

$$
\begin{array}{r}
0.02345700 \\
-0.02266500 \\
-0.09896600 \\
0.03278600 \\
-0.85833300 \\
0.92720200 \\
0.73065300
\end{array}
$$


Methyl peroxide

\begin{tabular}{|c|c|c|c|}
\hline \multicolumn{2}{|c|}{ MN15-L/CBSB7+ Enthalpy= } & \multicolumn{2}{|c|}{190.663747} \\
\hline $\mathrm{MN}$ & e Energy= & \multicolumn{2}{|c|}{-190.694495} \\
\hline 01 & & & \\
\hline $\mathrm{C}$ & -1.12153500 & -0.22234800 & 0.02796900 \\
\hline 0 & 0.01837900 & 0.60897100 & -0.03294700 \\
\hline 0 & 1.15137600 & -0.29008700 & -0.08789500 \\
\hline $\mathrm{H}$ & -1.97740700 & 0.47587000 & 0.00975000 \\
\hline $\mathrm{H}$ & -1.17116600 & -0.89686400 & -0.84902900 \\
\hline $\mathrm{H}$ & -1.13987500 & -0.82081800 & 0.96131700 \\
\hline $\mathrm{H}$ & 1.65961300 & 0.02483200 & 0.67688600 \\
\hline
\end{tabular}

Methyl peroxide

MN15-L/def2TZVP Enthalpy=

MN15-L/def2TZVP Free Energy=

01

C

o

O

$\mathrm{H}-1.15891800$

$\mathrm{H}-1.12331100$

$\mathrm{H} \quad 1.52549800$

-1.11184100
0.01619900
1.15953800
-1.97812000
-1.15891800
-1.12331100
1.52549800

$-0.22932200$

0.61551400

$-0.26120000$

0.45364400

$-0.86587100$

$-0.86963000$

$-0.17672400$
0.01637300
-0.01007600
-0.10939600
0.03523900
-0.88614600
0.92047600
0.78796500

Methyl peroxyl radical

CBS-QB3 Enthalpy = -189.954731

B3LYP/CBSB7 Enthalpy $=-190.224254$

02

$\mathrm{C}$
$\mathrm{O}$
$\mathrm{O}$
$\mathrm{H}$
$\mathrm{H}$
$\mathrm{H}$

1.09605600
-0.15733400
-1.18625500
1.87467300
1.14887400
1.14883600

CBS-QB3 Free Energy = -189.985244

B3LYP/CBSB7 Free Energy = -190.254744

Methyl peroxyl radical

\begin{tabular}{|c|c|c|c|}
\hline \multicolumn{2}{|c|}{ wB97X/CBSB7 Enthalpy= } & \multicolumn{2}{|c|}{-190.169120} \\
\hline wB & nergy $=$ & 0.199493 & \\
\hline 02 & & & \\
\hline $\mathrm{C}$ & 1.08492300 & -0.17998700 & -0.00000200 \\
\hline 0 & -0.16060600 & 0.53968400 & -0.00001000 \\
\hline 0 & -1.17065800 & -0.27757900 & 0.00001200 \\
\hline $\mathrm{H}$ & 1.86571700 & 0.57959900 & -0.00003700 \\
\hline $\mathrm{H}$ & 1.13743700 & -0.79823100 & 0.89697600 \\
\hline $\mathrm{H}$ & 1.13741400 & -0.79829100 & -0.89694100 \\
\hline
\end{tabular}

Methyl peroxyl radical

\begin{tabular}{|c|c|c|c|}
\hline M0 & nthalpy= & -190.1615 & \\
\hline & ree Energy= & -190.1920 & \\
\hline $\mathrm{C}$ & 1.08188300 & -0.17914900 & -0.00000300 \\
\hline 0 & -0.15975400 & 0.53734000 & -0.00001400 \\
\hline
\end{tabular}




$\begin{array}{rrrr}\mathrm{O} & -1.16771500 & -0.27604900 & 0.00001700 \\ \mathrm{H} & 1.86290300 & 0.57678600 & -0.00003500 \\ \mathrm{H} & 1.13278600 & -0.79607800 & 0.89490600 \\ \mathrm{H} & 1.13276800 & -0.79613700 & -0.89487300\end{array}$

Methyl peroxyl radical

$\begin{array}{lrrr}\text { M06-2X/CBSB7+ Enthalpy= } & -190.139606 & \\ \text { M06-2X/CBSB7+ Free Energy= } & -190.170035 & \\ 0 \text { 2 } & 1.08583000 & -0.17922700 & -0.00000300 \\ \text { C } & -0.15986200 & 0.53595000 & -0.00001400 \\ \text { O } & -1.17183400 & -0.27526500 & 0.00001600 \\ \text { O } & 1.86301200 & 0.58171900 & -0.00003600 \\ \text { H } & 1.13780000 & -0.79589100 & 0.89646600 \\ \text { H } & 1.13778200 & -0.79595000 & -0.89643200 \\ \text { H } & & & \end{array}$

Methyl peroxyl radical

$\begin{array}{lrrr}\text { M06-2X-D3/CBSB7 Enthalpy= } & -190.134194 & \\ \text { M06-2X-D3/CBSB7 Free Energy }= & -190.164598 & \\ \text { O 2 } & 1.08343500 & -0.17965500 & -0.00000200 \\ \text { C } & -0.15974400 & 0.53841900 & -0.00001100 \\ \text { O } & -1.16914800 & -0.27652400 & 0.00001300 \\ \text { O } & 1.86427000 & 0.57746300 & -0.00003700 \\ \text { H } & 1.13314200 & -0.79731300 & 0.89581200 \\ \text { H } & 1.13312000 & -0.79737200 & -0.89577700 \\ \text { H } & & & \end{array}$

Methyl peroxyl radical

M06-2X/CBSB7 Enthalpy= M06-2X/CBSB7 Free Energy= 02

C

O

0

$\mathrm{H}$

$\mathrm{H}$

$\mathrm{H}$
1.08342000

$-0.15974400$

$-1.16912200$

1.86424400

1.13309400

1.13307100
$-190.134186$

$-190.164590$

$$
\begin{array}{r}
-0.17966400 \\
0.53843300 \\
-0.27653100 \\
0.57745600 \\
-0.79731300 \\
-0.79737300
\end{array}
$$

\begin{tabular}{|c|c|c|c|}
\hline M0 & & 0.107983 & \\
\hline M0 & $\mathrm{rgy}=$ & 0.138341 & \\
\hline 02 & & & \\
\hline $\mathrm{C}$ & 1.08306200 & -0.17978300 & -0.00000200 \\
\hline 0 & -0.15907500 & 0.54066300 & -0.00000800 \\
\hline 0 & -1.17112900 & -0.27768800 & 0.00001000 \\
\hline $\mathrm{H}$ & 1.86762700 & 0.57694300 & -0.00003800 \\
\hline $\mathrm{H}$ & 1.13783200 & -0.80099100 & 0.89651400 \\
\hline $\mathrm{H}$ & 1.13780600 & -0.80105200 & -0.89647800 \\
\hline
\end{tabular}

Methyl peroxyl radical

Methyl peroxyl radical

$\begin{array}{ll}\text { M06/CBSB7+ Enthalpy }= & -190.113682 \\ \text { M06/CBSB7+ Free Energy }= & -190.144096\end{array}$


02

$\begin{array}{lr}\mathrm{C} & 1.08568100 \\ \mathrm{O} & -0.15924300 \\ \mathrm{O} & -1.17394400 \\ \mathrm{H} & 1.86656900 \\ \mathrm{H} & 1.14243100 \\ \mathrm{H} & 1.14240400\end{array}$

Methyl peroxyl radical

$$
\begin{array}{r}
-0.17936100 \\
0.53814300 \\
-0.27640200 \\
0.58110700 \\
-0.79940700 \\
-0.79946700
\end{array}
$$

-0.00000200
-0.00000800
0.00000900
-0.00003900
0.89713300
-0.89709600
$-190.138313$

$-190.168728$

$$
\begin{array}{r}
-0.17860900 \\
0.53976100 \\
-0.27772100 \\
0.57409600 \\
-0.79934800 \\
-0.79940700
\end{array}
$$

Methyl peroxyl radical
M06-L/CBSB7 Enthalpy= M06-L/CBSB7 Free Energy= 02

$\mathrm{C}$
$\mathrm{O}$
$\mathrm{O}$
$\mathrm{H}$
$\mathrm{H}$
$\mathrm{H}$

$$
\begin{array}{r}
1.09214500 \\
-0.15843000 \\
-1.18200300 \\
1.87342000 \\
1.14859600 \\
1.14857600
\end{array}
$$

Methyl peroxyl radical

M06-L/CBSB7+ Enthalpy $=$
M06-L/CBSB7+ Free Energy=

02

$\mathrm{C}$
$\mathrm{O}$
$\mathrm{O}$
$\mathrm{H}$
$\mathrm{H}$
$\mathrm{H}$

Methyl peroxyl radical

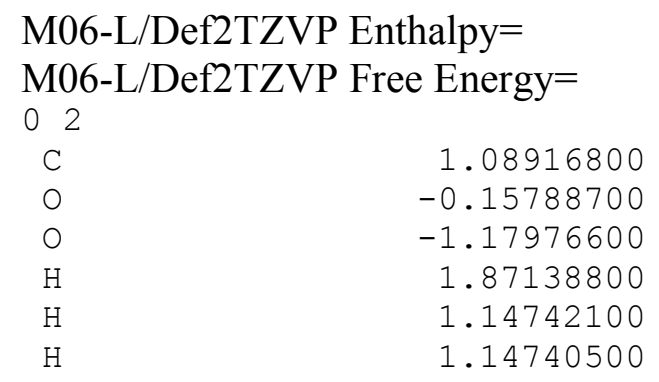

$-190.195013$

$-190.225510$
-0.00000300
-0.00001300
0.00001600
-0.00003600
0.89557100
-0.89553700
$-190.199070$

$-190.229595$

$$
\begin{array}{rr}
1.09395400 & -0.18191100 \\
-0.15824200 & 0.54301500 \\
-1.18396600 & -0.27873100 \\
1.87268500 & 0.57922500 \\
1.15066200 & -0.80097900 \\
1.15059000 & -0.80104800
\end{array}
$$

0.00000600
0.00002900
-0.00003300
-0.00005400
0.89590900
-0.89585500
$-0.00000300$

$-0.00001400$

0.00001700

$$
\begin{gathered}
-190.217532 \\
-190.248056 \\
-0.18132500 \\
0.54368900 \\
-0.27926400 \\
0.57303900 \\
-0.80021500 \\
-0.80027300
\end{gathered}
$$

Methyl peroxyl radical 
MN15/CBSB7 Free Energy=

02

$\begin{array}{lr}\mathrm{C} & 1.08108100 \\ \mathrm{O} & -0.15662000 \\ \mathrm{O} & -1.17081800 \\ \mathrm{H} & 1.86971000 \\ \mathrm{H} & 1.13166800 \\ \mathrm{H} & 1.13164500\end{array}$

Methyl peroxyl radical

\section{MN15/CBSB7+ Enthalpy $=\quad-190.017015$ \\ MN15/CBSB7+ Free Energy $=\quad-190.047475$}

02

$\begin{array}{lr}\mathrm{C} & 1.08381100 \\ \mathrm{O} & -0.15682300 \\ \mathrm{O} & -1.17369800 \\ \mathrm{H} & 1.86823700 \\ \mathrm{H} & 1.13654600 \\ \mathrm{H} & 1.13652300\end{array}$

Methyl peroxyl radical
$-190.041765$

$$
\begin{array}{rr}
-0.18071400 & -0.00000300 \\
0.54219100 & -0.00001100 \\
-0.27759400 & 0.00001400 \\
0.56889000 & -0.00003700 \\
-0.80066000 & 0.89498200 \\
-0.80071900 & -0.89494700
\end{array}
$$

$$
\begin{array}{r}
-0.18025300 \\
0.53944000 \\
-0.27620500 \\
0.57359700 \\
-0.79894800 \\
-0.79900700
\end{array}
$$

\section{MN15/ def2TZVP Enthalpy= $\quad-190.043338$ MN15/ def2TZVP Free Energy $=\quad-190.073805$} 02

$\begin{array}{lr}\mathrm{C} & 1.07852200 \\ \mathrm{O} & -0.15676100 \\ \mathrm{O} & -1.16792200 \\ \mathrm{H} & 1.86680400 \\ \mathrm{H} & 1.12977400 \\ \mathrm{H} & 1.12975500\end{array}$

Methyl peroxyl radical

$$
\begin{array}{rr}
-0.18018000 & -0.00000300 \\
0.54080800 & -0.00001400 \\
-0.27700900 & 0.00001700 \\
0.56833300 & -0.00003600 \\
-0.79879600 & 0.89420300 \\
-0.79885500 & -0.89416900
\end{array}
$$

\section{MN15-L/CBSB7 Enthalpy= MN15-L/CBSB7 Free Energy= \\ $-190.032054$ \\ $-190.062439$} 02

$\begin{array}{lr}\mathrm{C} & 1.08708500 \\ \mathrm{O} & -0.15754000 \\ \mathrm{O} & -1.17792300 \\ \mathrm{H} & 1.88235000 \\ \mathrm{H} & 1.13943300 \\ \mathrm{H} & 1.13941200\end{array}$

Methyl peroxyl radical

\section{MN15-L/CBSB7+ Enthalpy= MN15-L/CBSB7+ Free Energy=} 02

$\mathrm{C}$
$\mathrm{O}$
$\mathrm{O}$
$\mathrm{H}$
$\mathrm{H}$
$\mathrm{H}$

Methyl peroxyl radical

$$
\begin{array}{r}
-0.18286200 \\
0.54799800 \\
-0.28038500 \\
0.57938800 \\
-0.81153100 \\
-0.81159100
\end{array}
$$


MN15-L/def2TZVP Free Energy=

02

$\begin{array}{lr}\mathrm{C} & 1.08379500 \\ \mathrm{O} & -0.15570300 \\ \mathrm{O} & -1.17612400 \\ \mathrm{H} & 1.88123200 \\ \mathrm{H} & 1.13531700 \\ \mathrm{H} & 1.13529600\end{array}$

$-190.086890$

$$
\begin{array}{rr}
-0.18373600 & -0.00000300 \\
0.54931900 & -0.00001300 \\
-0.28058400 & 0.00001500 \\
0.57408700 & -0.00003700 \\
-0.81074800 & 0.90388500 \\
-0.81080800 & -0.90385000
\end{array}
$$

Hydrogen atom

CBS-QB3 Enthalpy $=-0.497457$

B3LYP/CBSB7 Enthalpy $=-0.499795$

wB97X/CBSB7 Enthalpy $=-0.512049$

M06-2X/Def2TZVP Enthalpy $=-0.508793$

M06-2X/CBSB7+ Enthalpy $=-0.508788$

M06-2X-D3/CBSB7 Enthalpy $=-0.508788$

M06-2X/CBSB7 Enthalpy $=-0.508788$

M06/CBSB7 Enthalpy $=-0.510531$

M06/CBSB7+ Enthalpy $=-0.510531$

M06/Def2TZVP Enthalpy $=-0.510524$

M06-L/CBSB7 Enthalpy $=-0.514082$

M06-L/CBSB7+ Enthalpy $=-0.514082$

M06-L/Def2TZVP Enthalpy $=-0.514078$

MN15/CBSB7 Enthalpy $=-0.509157$

MN15/CBSB7+ Enthalpy $=-0.509157$

MN15/ def2TZVP Enthalpy $=-0.509153$

MN15-L/CBSB7 Enthalpy $=-0.506274$

MN15-L/CBSB7+ Enthalpy $=-0.506274$

MN15-L/def2TZVP Enthalpy $=-0.506272$
CBS-QB3 Free Energy $=-0.510472$

B3LYP/CBSB7 Free Energy $=-0.512810$

$w B 97 X / C B S B 7$ Free Energy $=-0.499034$

M06-2X/Def2TZVP Free Energy $=-0.495778$

M06-2X/CBSB7+ Free Energy $=-0.495774$

M06-2X-D3/CBSB7 Free Energy $=-0.495774$

M06-2X/CBSB7 Free Energy $=-0.495774$

M06/CBSB7 Free Energy $=-0.497516$

M06/CBSB7+ Free Energy $=-0.497516$

M06/Def2TZVP Free Energy $=-0.497510$

M06-L/CBSB7 Free Energy $=-0.501067$

M06-L/CBSB7+ Free Energy $=-0.501067$

M06-L/Def2TZVP Free Energy $=-0.501064$

MN15/CBSB7 Free Energy $=-0.496143$

MN15/CBSB7+ Free Energy $=-0.496143$

MN15/ def2TZVP Free Energy $=-0.496139$

MN15-L/CBSB7 Free Energy $=-0.493259$

$\mathrm{MN15-L/CBSB7}+$ Free Energy $=-0.493259$

MN15-L/def2TZVP Free Energy $=-0.493257$
02

$\mathrm{H}$

0.00000000

0.00000000

0.00000000

Di-methoxy adduct derived from truncated CuATSM<smiles></smiles>

MN15/CBSB7+ Enthalpy=

$\mathrm{MN15/CBSB7+}$ Free Energy=

\section{2}

S

$\mathrm{N}$

$\mathrm{N}$

C

$\mathrm{N}$

$\mathrm{N}$

C

S

C

O

$$
\begin{array}{r}
-0.46223800 \\
-1.44813600 \\
-0.26368600 \\
1.66252900 \\
2.27729800 \\
3.64170000 \\
4.08075000 \\
3.19328500 \\
0.16242500 \\
-0.11733700
\end{array}
$$

$-3285.102227$

$-3285.176001$

$$
\begin{array}{r}
-1.71389600 \\
0.38590400 \\
0.67865100 \\
1.90349600 \\
0.94821300 \\
0.88599200 \\
-0.13118700 \\
-1.30163900 \\
1.94515800 \\
3.02286700
\end{array}
$$


3.32510800

3.27482800

2. 27124900

3.53143900

4.01351100

$-0.43502300$

$-0.83042400$

$-1.64090200$

$-1.92396900$

$-3.33432900$

$-3.71351600$

$-3.52086800$

$-3.79805700$

$-0.32228500$

$-0.83252000$

2.06978900

2.68996000

$-0.23532500$

0.08269000

0.70767200

0.67955800

$-0.76020100$
0.36934100

$-0.93115600$

$-1.35781600$

$-0.77902500$

$-1.58301100$

$-0.35976300$

$-0.29329700$

0.61559200

1.71396900

1.77200200

0.85967900

2.63116600

1.93345500

$-1.25611800$

$-2.12899800$

2.02521600

1.71432300

$-0.06660800$

$-0.82074500$

0.06891400

$-1.61089800$

$-0.57955500$

\section{Di-methoxy adduct derived from truncated CuATSM}<smiles></smiles>

B3LYP/CBSB7 Enthalpy= B3LYP/CBSB7 Free Energy=

02

$\mathrm{S}$

$\mathrm{N}$

$\mathrm{N}$

C

$\mathrm{N}$

$\mathrm{N}$

C

S

C

0

O

C

$\mathrm{H}$

$\mathrm{H}$

$\mathrm{Cu}$

C

0

O

C

$\mathrm{H}$

$\mathrm{H}$

$\mathrm{H}$

N

$\mathrm{H}$

$\mathrm{H}$

$\mathrm{H}$

\begin{abstract}
0.53745300
1. 46115600

0.27514600

$-1.69106000$

$-2.28048900$

$-3.63747500$

$-4.05122300$

$-3.13193100$

$-0.20062300$

$-0.03810400$

1. 34544900

1.87407100

1.95163400

2. 87102300

1. 27884000

$-1.14726400$

1.88170600

2. 51118100

1.54774100

1. 45728500

1.05642300

0.76456600

2.43150700

2.88164400

2.83651500

0.35074100

$-2.24068300$
\end{abstract}

$-3285.872023$

$-3285.946721$

$$
\begin{array}{r}
-1.62407000 \\
0.41818700 \\
0.70135700 \\
1.81381000 \\
0.87619900 \\
0.73825300 \\
-0.26642900 \\
-1.36099100 \\
1.95085400 \\
3.04723600 \\
3.50524100 \\
3.55320300 \\
2.55483900 \\
3.97772100 \\
4.21520500 \\
-0.41312100 \\
-0.80155700 \\
-1.65861400 \\
-2.01922800 \\
-3.44048800 \\
-3.79717600 \\
-3.66913700 \\
-3.89222300 \\
-0.29476200 \\
-0.74702500 \\
2.11732300 \\
2.52911400
\end{array}
$$

1. 22079700

$-0.54511000$

$-0.43670200$

$-1.28001500$

$-0.63474300$

$-0.70840100$

0.02258800

0.98293000

$-1.09661300$

$-0.22855200$

$-0.34871900$

0.97336100

1. 41076100

0.83432900

1. 60757600

0.39435400

0.26389600

$-0.67487600$

$-1.70896500$

$-1.69466300$

$-0.74416000$

$-2.50824200$

$-1.90261000$

1. 13694000

2. 03870800

$-2.02632200$

$-1.88605500$ 


$\begin{array}{rrrr}\mathrm{H} & -5.12751800 & -0.41849200 & 0.01255400 \\ \mathrm{C} & 4.23520500 & -0.03566100 & 0.63536000 \\ \mathrm{H} & 4.17363500 & 0.54826200 & -0.28243200 \\ \mathrm{H} & 4.76726500 & 0.55528200 & 1.38363700 \\ \mathrm{H} & 4.80619300 & -0.94630600 & 0.42787100\end{array}$

Truncated analogue of CuATSM<smiles>NC1=NN2CCN3N=CSC32S1</smiles>

\section{CBS-QB3 Enthalpy= \\ CBS-QB3 Free Energy= \\ B3LYP/CBSB7 Enthalpy= \\ B3LYP/CBSB7 Free Energy=}

$-2863.437549$

$-2863.489769$

$-2866.103388$

$-2866.155427$

$\mathrm{O}^{2} 2$
$\mathrm{~S}$
$\mathrm{C}$
$\mathrm{N}$
$\mathrm{N}$
$\mathrm{C}$
$\mathrm{N}$
$\mathrm{N}$
$\mathrm{C}$
$\mathrm{S}$
$\mathrm{C}$
$\mathrm{N}$
$\mathrm{H}$
$\mathrm{Cu}$
$\mathrm{H}$
$\mathrm{H}$
$\mathrm{H}$
$\mathrm{H}$

$$
\begin{array}{r}
1.67296700 \\
2.68638000 \\
2.27885000 \\
0.95571800 \\
-1.12389300 \\
-1.61917500 \\
-2.96144300 \\
-3.21587500 \\
-2.09318900 \\
0.32515800 \\
4.01742700 \\
4.41185400 \\
-0.27072900 \\
-4.27001300 \\
-1.76703600 \\
0.85275700 \\
4.61685200
\end{array}
$$

0.00000800

0.00002200

0.00001000

0.00000400

$-0.00000800$

$-0.00000900$

$-0.00001300$

$-0.00000100$

0.00000300

$-0.00000200$

$-0.00001800$

$-0.00003700$

0.00000100

$-0.00002600$

$-0.00001100$

$-0.00000300$

$-0.00003100$

\section{Truncated analogue of CuATSM}<smiles>NC1=NN2CCN3N=CSC32S1</smiles>

\section{MN15/CBSB7+ Enthalpy= MN15/CBSB7+ Free Energy=}

$$
\begin{array}{r}
-1.66809000 \\
-2.67478500 \\
-2.27751000 \\
-0.95296100 \\
1.13304000 \\
1.62003000 \\
2.96436300 \\
3.20624100 \\
2.07733700 \\
-0.32334600
\end{array}
$$

$$
\begin{array}{r}
-1.48810600 \\
-0.08186300 \\
1.19410900 \\
1.28741200 \\
2.31185000 \\
1.12035400 \\
0.88855600 \\
-0.38931700 \\
-1.68423300 \\
2.41854300
\end{array}
$$




$\begin{array}{lrrr}\mathrm{N} & -4.00555400 & -0.27223300 & 0.00001100 \\ \mathrm{H} & -4.38768400 & -1.20519900 & 0.00002800 \\ \mathrm{Cu} & 0.26816300 & -0.32884800 & -0.00000200 \\ \mathrm{H} & 4.25777100 & -0.66508700 & -0.00001800 \\ \mathrm{H} & 1.78569400 & 3.18138400 & 0.00000400 \\ \mathrm{H} & -0.84885400 & 3.36788800 & 0.00000000 \\ \mathrm{H} & -4.61708400 & 0.53236600 & 0.00002200\end{array}$

TS structure of peroxyl-addition to truncated CuATSM<smiles>COC1=CN2N=CSC23SC(N)=NN13</smiles>

CBS-QB3 Enthalpy=

CBS-QB3 Free Energy=

B3LYP/CBSB7 Enthalpy= B3LYP/CBSB7 Free Energy= Imaginary frequency $=$

03

$\mathrm{Cu}$
$\mathrm{S}$
$\mathrm{C}$
$\mathrm{N}$
$\mathrm{N}$
$\mathrm{C}$
$\mathrm{N}$
$\mathrm{N}$
$\mathrm{C}$
$\mathrm{S}$
$\mathrm{C}$
$\mathrm{O}$
$\mathrm{O}$
$\mathrm{C}$
$\mathrm{H}$
$\mathrm{H}$
$\mathrm{H}$
$\mathrm{N}$
$\mathrm{H}$
$\mathrm{H}$
$\mathrm{H}$
$\mathrm{H}$
$\mathrm{H}$

$$
\begin{array}{r}
1.00500900 \\
0.25561000 \\
-1.32963800 \\
-1.77113000 \\
-0.84146300 \\
0.10692900 \\
1.17748600 \\
2.34646100 \\
3.28623500 \\
3.19640500 \\
-1.09012000 \\
-1.92016300 \\
-3.25182400 \\
-4.03337600 \\
-3.84231600 \\
-5.07112200 \\
-3.79443200 \\
-2.22606700 \\
-1.97248000 \\
4.24979100 \\
0.07880800 \\
-3.13367400 \\
-1.95339100
\end{array}
$$

$-3053.389701$ $-3053.453800$ $-3056.320288$ $-3056.384140$ $-446.77$

$$
\begin{array}{rr}
0.41154400 & 0.02910900 \\
2.55147100 & 0.34074400 \\
2.20690700 & -0.29151300 \\
1.00983100 & -0.72180400 \\
0.07465400 & -0.61748000 \\
-2.03612300 & -0.95460000 \\
-1.49205200 & -0.49002000 \\
-2.18718200 & -0.36868900 \\
-1.43449300 & 0.13176300 \\
0.22897400 & 0.61719300 \\
-1.18809400 & -0.96901200 \\
-2.10022600 & 0.60110100 \\
-2.33054500 & 0.38069400 \\
-1.32882400 & 1.04932700 \\
-0.34849000 & 0.60828100 \\
-1.62618100 & 0.89144200 \\
-1.32179900 & 2.11478000 \\
3.20552300 & -0.37087900 \\
4.14086300 & -0.10291000 \\
-1.92042800 & 0.25856500 \\
-3.07569100 & -1.26577400 \\
3.01956600 & -0.76853300 \\
-1.43521500 & -1.57504500
\end{array}
$$

TS structure of peroxyl-addition to truncated CuATSM<smiles>COC1=CN2N=CSC23SC(N)=NN13</smiles>

MN15/CBSB7+ Enthalpy= 
Peroxyl-adduct derived from truncated CuATSM<smiles></smiles>

CBS-QB3 Enthalpy= CBS-QB3 Free Energy= B3LYP/CBSB7 Enthalpy= B3LYP/CBSB7 Free Energy=

03

S
C
N
N
C
N
N
C
S
C
O
O
C
H
H
H
N
H
Cu
H
H
H
H

$$
0.23839800
$$

$-1.37980400$

$-1.79482600$

$-0.83566200$

0.14951200

1.21081300

2.42639400

3.37075900

3.25457000

$-1.15516800$

$-1.96223500$

$-3.35821900$

$-4.03792200$

$-3.67346000$

$-5.08141500$

$-3.95085900$

$-2.32707500$

$-2.08904500$

1.01448000

4.36252600

0.16388100

$-1.66988500$

$-3.24729000$
$-3053.426000$

$-3053.489230$

$-3056.345271$

$-3056.408253$

$$
\begin{array}{rr}
2.55382700 & 0.19909600 \\
2.10590800 & -0.24865500 \\
0.84792600 & -0.50335200 \\
-0.03646000 & -0.36222500 \\
-2.19151400 & -0.59631500 \\
-1.54355200 & -0.29525400 \\
-2.17349400 & -0.22335400 \\
-1.33259400 & 0.09911800 \\
0.36521300 & 0.41098800 \\
-1.42579200 & -0.62900000 \\
-2.04497700 & 0.36816500 \\
-1.91904000 & -0.03922900 \\
-1.27518100 & 1.02925000 \\
-0.25527000 & 1.17160300 \\
-1.25993900 & 0.70507900 \\
-1.85143300 & 1.95477000 \\
3.05769600 & -0.35939300 \\
4.02811300 & -0.24734600 \\
0.40572400 & 0.04056600 \\
-1.76976400 & 0.18000300 \\
-3.26147900 & -0.78668300 \\
-1.53405200 & -1.59120600 \\
2.79555400 & -0.67681500
\end{array}
$$

Peroxyl-adduct derived from truncated CuATSM<smiles>COC1CN2N=CSC23SC(N)=NN13</smiles>

MN15/CBSB7+ Enthalpy $=$
MN15/CBSB7+ Free Energy=

03

$\mathrm{S}$

C

$\mathrm{N}$

$\mathrm{N}$

$$
\begin{array}{r}
0.12182000 \\
-1.45812900 \\
-1.82719100 \\
-0.83434300
\end{array}
$$

$-3055.817503$ $-3055.879999$

$$
\begin{array}{r}
2.52867500 \\
2.03079800 \\
0.75817700 \\
-0.08043700
\end{array}
$$




$\begin{array}{lrrr}\mathrm{C} & 0.22726900 & -2.19539600 & -0.57850700 \\ \mathrm{~N} & 1.26348100 & -1.50536300 & -0.29466800 \\ \mathrm{~N} & 2.49688700 & -2.09483700 & -0.21001400 \\ \mathrm{C} & 3.40461500 & -1.21973000 & 0.11357900 \\ \mathrm{~S} & 3.21735400 & 0.45634300 & 0.41029400 \\ \mathrm{C} & -1.09714300 & -1.47588900 & -0.64000800 \\ \mathrm{O} & -1.90131900 & -2.11028900 & 0.33030600 \\ \mathrm{O} & -3.26664500 & -1.91000500 & -0.04150200 \\ \mathrm{C} & -3.89972200 & -1.31816300 & 1.07545500 \\ \mathrm{H} & -3.46778600 & -0.33804100 & 1.29024900 \\ \mathrm{H} & -4.94395900 & -1.21237000 & 0.77875500 \\ \mathrm{H} & -3.82875300 & -1.96967100 & 1.94964500 \\ \mathrm{~N} & -2.44231100 & 2.94572700 & -0.35721200 \\ \mathrm{H} & -2.24653200 & 3.92514700 & -0.21892100 \\ \mathrm{Cu} & 0.99985500 & 0.43931300 & 0.03696900 \\ \mathrm{H} & 4.41253400 & -1.61729400 & 0.20474200 \\ \mathrm{H} & 0.28294400 & -3.26846400 & -0.74329300 \\ \mathrm{H} & -1.58363300 & -1.59593100 & -1.61698100 \\ \mathrm{H} & -3.36069100 & 2.64603800 & -0.65358200\end{array}$

Imine derived from truncated peroxyl-substituted CuATSM<smiles></smiles>

\section{CBS-QB3 Enthalpy= CBS-QB3 Free Energy= B3LYP/CBSB7 Enthalpy= B3LYP/CBSB7 Free Energy=}

$\begin{array}{lr}02 & \\ \mathrm{Cu} & 0.95351500 \\ \mathrm{~S} & 0.21752300 \\ \mathrm{C} & -1.38244100 \\ \mathrm{~N} & -1.76099100 \\ \mathrm{~N} & -0.90286200 \\ \mathrm{C} & 0.11815600 \\ \mathrm{~N} & 1.15377300 \\ \mathrm{~N} & 2.36876800 \\ \mathrm{C} & 3.30187200 \\ \mathrm{~S} & 3.17192700 \\ \mathrm{~N} & -2.26308900 \\ \mathrm{C} & -1.20319500 \\ \mathrm{O} & -1.90117400 \\ \mathrm{O} & -3.31689800 \\ \mathrm{C} & -3.82350900 \\ \mathrm{H} & -3.34020400 \\ \mathrm{H} & -4.88220300 \\ \mathrm{H} & -3.71733900 \\ \mathrm{H} & 0.16488400 \\ \mathrm{H} & -1.79103000 \\ \mathrm{H} & 4.29223000 \\ \mathrm{H} & -3.09937700\end{array}$

$-3052.806473$ $-3052.869024$ $-3055.718424$ $-3055.780738$

$$
\begin{array}{r}
0.41996500 \\
2.52919100 \\
2.23217900 \\
0.86731000 \\
0.02488100 \\
-2.12772200 \\
-1.50550400 \\
-2.12655100 \\
-1.32123800 \\
0.32369300 \\
3.13277100 \\
-1.40169400 \\
-1.98301800 \\
-1.65252600 \\
-1.36737900 \\
-0.48825700 \\
-1.16444700 \\
-2.23044100 \\
-3.15919700 \\
-1.48131300 \\
-1.76528700 \\
2.71294100
\end{array}
$$

Imine derived from truncated peroxyl-substituted CuATSM

$$
\begin{array}{r}
0.09371600 \\
0.30728100 \\
-0.37354500 \\
-0.76246800 \\
-0.48508800 \\
-0.83535000 \\
-0.40582200 \\
-0.37828300 \\
0.06524100 \\
0.55809000 \\
-0.55489800 \\
-0.78012700 \\
0.29238700 \\
0.10995900 \\
1.40698000 \\
1.84077700 \\
1.23246300 \\
2.06956100 \\
-1.17416500 \\
-1.69982000 \\
0.12354100 \\
-0.96809900
\end{array}
$$


<smiles></smiles>

MN15/CBSB7+ Enthalpy=

MN15/CBSB7+ Free Energy=

02

$\mathrm{Cu}$

$\mathrm{S}$

C

$\mathrm{N}$

N

C

$\mathrm{N}$

$\mathrm{N}$

C

S

$\mathrm{N}$

C

O

0

C

$\mathrm{H}$

H

$\mathrm{H}$

$\mathrm{H}$

$\mathrm{H}$

$\mathrm{H}$

$\mathrm{H}$
0.88522800

$-0.09167100$

$-1.62774000$

$-1.84580400$

$-0.91544300$

0.32978600

1.29033800

2. 56434400

3. 39419800

3.07509900

$-2.61510500$

$-1.05892200$

$-1.67158000$

$-3.06463800$

$-3.40390600$

$-2.84832400$

$-4.47044600$

$-3.21982400$

0.49166800

$-1.65278400$

4.42927500

$-3.39509900$
$-3055.194257$

$-3055.256129$

0.48657800

2.48484000

2.01178600

0.61990100

$-0.12075700$

$-2.14605700$

$-1.40692900$

$-1.89423800$

$-0.99507300$

0.60421700

2.79623400

$-1.56452200$

$-2.17524200$

$-1.86147300$

$-1.28256400$

$-0.35558800$

$-1.07076000$

$-1.98656600$

$-3.17323100$

$-1.72335900$

$-1.32230700$

2. 28898100
0.09603000

0.24155200

$-0.42820000$

$-0.83067300$

$-0.51228000$

$-0.83169100$

$-0.42288100$

$-0.38701900$

0.06955500

0.57243200

$-0.59160200$

$-0.78017200$

0.32055800

0.24915100

1. 49806300

1.66575400

1. 42225200

2. 31180900

$-1.14765400$

$-1.68619900$

0.13308600

$-1.01949200$

Di-peroxyl adduct derived from truncated CuATSM<smiles>COC1CN2N=CS[C@]23SC(N)(OC)NN13</smiles>

CBS-QB3 Enthalpy=

CBS-QB3 Free Energy=

B3LYP/CBSB7 Enthalpy=

B3LYP/CBSB7 Free Energy=

02

S
N
N
C
N
N
C
S
C
O
O
C
H
H

S
N
N
C
N
N
C
S
C
O
O
C
H
H

$-3243.425084$

$-3243.496726$

$-3246.588373$

$-3246.659700$

$$
-0.69987400
$$$$
-1.68336000
$$

0.49319700

0.72096400

1.78143100

0.80700100

0.63053800

$-0.41122100$

$-1.51119800$

1.95976800

3.05909800

3.57588300

3.64686900

2.65349600

4. 11728700

-1.19022700
0.39753400
0.35760100
1.27077000
0.67683800
0.80926500
0.13058400
-0.82705800
1.03265500
0.16166300
0.26493800
-1.06245700
-1.50070300
-0.93046300 


$\begin{array}{lrrr}\mathrm{H} & -1.30080900 & 4.28189200 & -1.69063600 \\ \mathrm{Cu} & 0.98435100 & -0.47786600 & -0.35709400 \\ \mathrm{C} & -2.06336200 & -0.71249500 & -0.42433300 \\ \mathrm{O} & -2.88907900 & -1.47023600 & 0.44350900 \\ \mathrm{O} & -2.10755200 & -1.88306700 & 1.60563800 \\ \mathrm{C} & -2.13798400 & -3.30669300 & 1.62764500 \\ \mathrm{H} & -1.64285300 & -3.72267700 & 0.74831000 \\ \mathrm{H} & -1.58476900 & -3.56848800 & 2.53287700 \\ \mathrm{H} & -3.16640400 & -3.67089200 & 1.70655600 \\ \mathrm{~N} & -2.92302300 & -0.14730800 & -1.41097900 \\ \mathrm{H} & -2.92454100 & -0.69725100 & -2.25903400 \\ \mathrm{H} & -3.85947000 & 0.00553900 & -1.04986400 \\ \mathrm{H} & -0.52590300 & 2.14060000 & 1.94295800 \\ \mathrm{H} & 2.08001400 & 2.49691900 & 1.87692900 \\ \mathrm{H} & 4.94229700 & -0.59549700 & 0.18836400\end{array}$

\section{Di-peroxyl adduct derived from truncated CuATSM}<smiles>COC1CN2N=CSC23SC(N)(OC)NN13</smiles>

\section{MN15/CBSB7+ Enthalpy= MN15/CBSB7+ Free Energy=}

$\begin{array}{lr}02 & -0.41422300 \\ \mathrm{~S} & -1.76977100 \\ \mathrm{~N} & -0.95607000 \\ \mathrm{~N} & 0.28073600 \\ \mathrm{C} & 1.21487700 \\ \mathrm{~N} & 2.49290500 \\ \mathrm{~N} & 3.29655600 \\ \mathrm{C} & 2.94653700 \\ \mathrm{~S} & -1.11243400 \\ \mathrm{C} & -1.74458600 \\ \mathrm{O} & -3.10889400 \\ \mathrm{O} & -3.34540900 \\ \mathrm{C} & -2.69917800 \\ \mathrm{H} & -4.38823700 \\ \mathrm{H} & -3.20316900 \\ \mathrm{H} & 0.77132300 \\ \mathrm{Cu} & -1.39312900 \\ \mathrm{C} & -0.69764500 \\ \mathrm{O} & 0.53211000 \\ \mathrm{O} & 1.57080000 \\ \mathrm{C} & 1.55738000 \\ \mathrm{H} & 2.49374200 \\ \mathrm{H} & 1.46110900 \\ \mathrm{H} & -2.59744000 \\ \mathrm{~N} & -2.46581800 \\ \mathrm{H} & -3.07090700 \\ \mathrm{H} & -1.69105700 \\ \mathrm{H} & 0.46756900 \\ \mathrm{H} & 4.33107300 \\ \mathrm{H} & \\ & \\ \mathrm{H} & \end{array}$

\section{$-3245.874905$ $-3245.944036$}

$$
\begin{array}{r}
-1.42754800 \\
0.73843000 \\
0.47722100 \\
1.22901600 \\
0.57983200 \\
0.63509600 \\
-0.09707400 \\
-1.04215800 \\
1.07476400 \\
0.13811500 \\
0.07638300 \\
-1.26759200 \\
-1.55614900 \\
-1.27827400 \\
-1.94452400 \\
-0.47914600 \\
0.07127700 \\
1.14021700 \\
1.40087000 \\
1.18048700 \\
0.14149100 \\
1.38737700 \\
1.86762500 \\
-0.15511300 \\
-0.76077600 \\
0.71074200 \\
2.00233400 \\
1.82237000 \\
-0.09987000
\end{array}
$$

Aminyl radical derived from truncated CuATSM 
<smiles>NC1=NN2C=CN3N=CS[C@@]32S1</smiles>

CBS-QB3 Enthalpy=

$-2862.783856$

CBS-QB3 Free Energy= $-2862.835545$

B3LYP/CBSB7 Enthalpy= $-2865.450666$ B3LYP/CBSB7 Free Energy=

$-2865.502185$

${ }^{0} 3$
S
C
$\mathrm{N}$
$\mathrm{N}$
$\mathrm{C}$
$\mathrm{N}$
$\mathrm{N}$
$\mathrm{C}$
$\mathrm{S}$
$\mathrm{C}$
$\mathrm{N}$
$\mathrm{Cu}$
$\mathrm{H}$
$\mathrm{H}$
$\mathrm{H}$
$\mathrm{H}$

$$
\begin{array}{r}
-1.62177100 \\
-2.77459400 \\
-2.28970500 \\
-1.03080500 \\
1.00286000 \\
1.53213900 \\
2.84402000 \\
3.17593700 \\
2.11329400 \\
-0.38502200 \\
-4.03349000 \\
0.22179900 \\
4.24235200 \\
1.64900700 \\
-0.95029500
\end{array}
$$$$
-1.56031000
$$$$
-0.19555700
$$$$
1.16133200
$$$$
1.23745700
$$$$
2.36605900
$$$$
1.13562300
$$$$
0.96291000
$$$$
-0.32579300
$$$$
-1.65988900
$$$$
2.44553500
$$$$
-0.38221900
$$$$
-0.33203300
$$$$
-0.52661800
$$$$
3.23811300
$$$$
3.36921900
$$$$
0.52426300
$$

$$
\begin{array}{r}
-0.00000100 \\
-0.00000200 \\
0.00000000 \\
0.00000100 \\
0.00000300 \\
0.00000300 \\
0.00000300 \\
0.00000300 \\
-0.00000200 \\
0.00000200 \\
-0.00000500 \\
0.00000000 \\
-0.00000100 \\
0.00000400 \\
0.00000300 \\
-0.00000500
\end{array}
$$

\section{Aminyl radical derived from truncated CuATSM}<smiles>NC1=NN2C=CN3N=CSC32S1</smiles>

\section{MN15/CBSB7+ Enthalpy= MN15/CBSB7+ Free Energy=}

$\mathrm{O}^{3}$
$\mathrm{~S}$
$\mathrm{C}$
$\mathrm{N}$
$\mathrm{N}$
$\mathrm{C}$
$\mathrm{N}$
$\mathrm{N}$
$\mathrm{C}$
$\mathrm{S}$
$\mathrm{C}$
$\mathrm{N}$
$\mathrm{Cu}$
$\mathrm{H}$
$\mathrm{H}$
$\mathrm{H}$
$\mathrm{H}$

$-2865.116478$

$-2865.168571$

$$
\begin{array}{r}
-1.54301200 \\
-0.19973600 \\
1.16003700 \\
1.23277200 \\
2.36520900 \\
1.13061300 \\
0.95881700 \\
-0.33343200 \\
-1.64282700 \\
2.44741800 \\
-0.37582300 \\
-0.34624100 \\
-0.53734600 \\
3.23228000 \\
3.36634100 \\
0.53149700
\end{array}
$$

Truncated analogue of CuATSM

-0.00000200
-0.00000700
-0.00000100
0.00000000
0.00000300
0.00000300
0.00000400
0.00000900
-0.00000100
0.00000100
-0.00000400
0.00000000
-0.00000600
0.00000400
0.00000200
0.00000000 
<smiles>CC1=CN2N=CS[C@]23SC(N)=NN13</smiles>

CBS-QB3 Enthalpy=

CBS-QB3 Free Energy=

B3LYP/CBSB7 Enthalpy= B3LYP/CBSB7 Free Energy=
$-2902.673633$

$-2902.730518$

$-2905.407708$

$-2905.464384$

02

\footnotetext{
S

C

$\mathrm{N}$

$\mathrm{N}$

C

$\mathrm{N}$

$\mathrm{N}$

C

S

C

$\mathrm{N}$

$\mathrm{H}$

$\mathrm{Cu}$

$\mathrm{H}$

$\mathrm{H}$

C

$\mathrm{H}$

$\mathrm{H}$

$\mathrm{H}$

$\mathrm{H}$
}

$$
\begin{array}{r}
-1.31224100 \\
-2.53449500 \\
-2.32748200 \\
-1.02601600 \\
0.85599500 \\
1.52840000 \\
2.89026500 \\
3.33890500 \\
2.43530100 \\
-0.60170700 \\
-3.82284000 \\
-4.06431700 \\
0.41904900 \\
4.42123200 \\
1.35972400 \\
-1.51126300 \\
-2.16478700 \\
-2.16478500 \\
-0.94537700 \\
-4.54397200
\end{array}
$$

\section{Truncated analogue of CuATSM}<smiles>CC1=CN2N=CS[C@]23SC(N)=NN13</smiles>

\section{MN15/CBSB7+ Enthalpy= MN15/CBSB7+ Free Energy=}

$\mathrm{O}^{2}$
$\mathrm{~S}$
$\mathrm{C}$
$\mathrm{N}$
$\mathrm{N}$
$\mathrm{C}$
$\mathrm{N}$
$\mathrm{N}$
$\mathrm{C}$
$\mathrm{S}$
$\mathrm{C}$
$\mathrm{N}$
$\mathrm{H}$
$\mathrm{Cu}$
$\mathrm{H}$
$\mathrm{H}$
$\mathrm{C}$

$$
\begin{array}{r}
-1.97529800 \\
-0.71863900 \\
0.60226500 \\
0.89868300 \\
2.24530300 \\
1.14257300 \\
1.12334100 \\
-0.10312100 \\
-1.58269800 \\
2.13337200 \\
-1.10282600 \\
-2.07840900 \\
-0.49546600 \\
-0.19919600 \\
3.20923500 \\
3.32048000 \\
3.29899500 \\
3.29899500 \\
4.25281800 \\
-0.39861600
\end{array}
$$

0.00000100
-0.00000500
-0.00000300
-0.00000100
0.00000200
0.00000400
0.00000400
-0.00000200
-0.00000600
0.00000100
0.00000700
0.00001000
0.00000000
0.00000400
0.00000100
0.00000200
-0.87671700
0.87672100
0.00000100
0.00000600

(1)




$\begin{array}{rrrr}\mathrm{H} & -2.15680600 & 3.27706900 & -0.87635400 \\ \mathrm{H} & -2.15680000 & 3.27707100 & 0.87636100 \\ \mathrm{H} & -0.94239600 & 4.24150400 & -0.00000200 \\ \mathrm{H} & -4.54484500 & -0.40338700 & -0.00000400\end{array}$

TS structure for HAT of truncated CuATSM

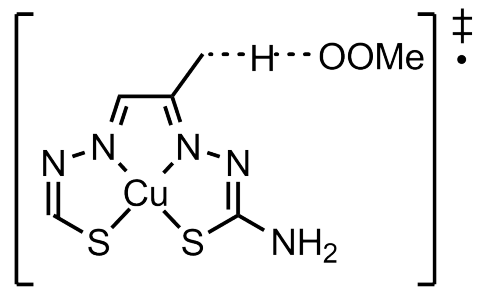

CBS-QB3 Enthalpy=

CBS-QB3 Free Energy=

B3LYP/CBSB7 Enthalpy=

B3LYP/CBSB7 Free Energy=

Imaginary frequency $=$
1. 17165500

3.30010700

3. 16777600

2.14726100

1.09985800

$-0.02726300$

$-1.07709600$

$-0.69652800$

$-1.51691500$

$-0.93568900$

0.69001500

$-1.71778000$

$-2.36964600$

$-2.40684300$

$-2.93920800$

$-3.05528100$

$-3.61398400$

$-2.66039300$

$-2.96247900$

$-3.96477900$

$-2.21164400$

$-2.88653100$

4.04499800

$-0.18845300$

$-1.35579200$

$-2.64628300$
$-3092.617344$

$-3092.684790$

$-3095.618579$

$-3095.685749$

$-1778.46$
0.35442100

$-0.10766700$

$-1.78464200$

$-2.41478200$

$-1.56500700$

$-1.97218000$

$-0.96309500$

0.24304100

1.28370400

2.41078900

2.56392300

3. 50353700

$-1.29022600$

$-2.18199200$

$-0.44783000$

$-1.67064000$

$-2.02027900$

$-1.73698700$

$-0.47740300$

$-0.50474700$

$-0.35051200$

0.32812600

$-2.39840400$

$-3.01617600$

4.39691300

3. 43216900
-0.02797000
0.65008900
0.22796900
-0.28565100
-0.48669900
-0.96068400
-1.14157300
-0.71396200
-0.76815000
-0.31829100
0.28882000
-0.34610300
-1.65841800
-2.28103200
-2.03888400
-0.62104900
0.45461700
1.41328000
2.02392400
2.45629700
2.80541400
1.28743800
0.41320900
-1.21680500
-0.05991900
-0.73285800

TS structure for HAT of truncated CuATSM

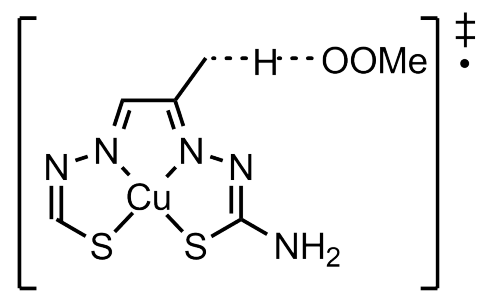

MN15/CBSB7+ Enthalpy=

$-3095.025091$

MN15/CBSB7+ Free Energy= $-3095.091949$

Imaginary frequency $=$ $-2001.82$

03 


$\begin{array}{lrrr}\mathrm{Cu} & 0.96164400 & 0.56364900 & -0.10310400 \\ \mathrm{~S} & 3.12146400 & 0.50711000 & 0.53375300 \\ \mathrm{C} & 3.34483900 & -1.13344700 & 0.07103900 \\ \mathrm{~N} & 2.47348100 & -1.94810900 & -0.44393100 \\ \mathrm{~N} & 1.26832300 & -1.32513500 & -0.60203800 \\ \mathrm{C} & 0.22689300 & -1.95824800 & -1.00467700 \\ \mathrm{C} & -1.01950300 & -1.18503100 & -1.12246600 \\ \mathrm{~N} & -0.86204800 & 0.08585300 & -0.76406900 \\ \mathrm{~N} & -1.87909800 & 0.93329700 & -0.77442700 \\ \mathrm{C} & -1.52822100 & 2.13570000 & -0.29184700 \\ \mathrm{~S} & 0.01642100 & 2.57664800 & 0.32545200 \\ \mathrm{~N} & -2.50574100 & 3.05969400 & -0.28861400 \\ \mathrm{C} & -2.26596600 & -1.79275500 & -1.46262700 \\ \mathrm{H} & -2.19516300 & -2.70719500 & -2.04762900 \\ \mathrm{H} & -3.04689200 & -1.10522000 & -1.77383000 \\ \mathrm{H} & -2.65198000 & -2.23178300 & -0.32161500 \\ \mathrm{O} & -2.71427900 & -2.59320500 & 0.92189600 \\ \mathrm{O} & -1.56293700 & -2.05550300 & 1.40281400 \\ \mathrm{C} & -1.85339000 & -0.84670300 & 2.09612500 \\ \mathrm{H} & -2.40205100 & -1.07004400 & 3.01270600 \\ \mathrm{H} & -0.88917600 & -0.38985000 & 2.32705900 \\ \mathrm{H} & -2.44228900 & -0.18572900 & 1.45390600 \\ \mathrm{H} & 4.33667500 & -1.54927800 & 0.22905000 \\ \mathrm{H} & 0.26360800 & -3.02124600 & -1.23165700 \\ \mathrm{H} & -2.32634800 & 3.99151300 & 0.05267700 \\ \mathrm{H} & -3.41479500 & 2.81626900 & -0.65732900 \\ & & & \end{array}$

\section{Methylene derived from truncated CuATSM}<smiles>C=C1CN2N=CSC23SC(N)=NN13</smiles>

CBS-QB3 Enthalpy=

CBS-QB3 Free Energy=

B3LYP/CBSB7 Enthalpy= B3LYP/CBSB7 Free Energy=

\begin{abstract}
$-2902.050518$
$-2902.105542$

$-2904.784742$

$-2904.839563$
\end{abstract}

\footnotetext{
03

$\mathrm{S}$

C

$\mathrm{N}$

$\mathrm{N}$

C

$\mathrm{N}$

$\mathrm{N}$

C

S

C

$\mathrm{N}$

$\mathrm{H}$

$\mathrm{Cu}$

$\mathrm{H}$

$\mathrm{H}$

C

$\mathrm{H}$

$\mathrm{H}$

$\mathrm{H}$
}

\begin{abstract}
$-1.43218300$
$-2.58194700$

$-2.28070400$

$-0.98307700$

0.97458600

1. 56175300

2. 92552600

3. 29858900

2. 30908200

$-0.49385700$

$-3.89670100$

$-4.21434700$

0.37257500

4.37332600

1.54446700

$-1.23148300$

$-2.31081900$

$-0.74947700$

$-4.56109600$
\end{abstract}

0.00000500
0.00000100
-0.00000100
-0.00000100
-0.00000100
0.00000100
0.00000000
-0.00000200
-0.00000400
-0.00000200
0.00000300
0.00000400
0.00000000
0.00000100
-0.00000200
-0.00000400
0.00000900
0.00000300
0.00000000

0.72973100

0.94393400

2.24585200

1.10070700

0.99674000

$-0.25270500$

$-1.67650300$

2.24260500

$-0.86759700$

$-1.82145500$

$-0.48139900$

$-0.41307300$

3.17201200

3.37874600

3. 33135500

4.34723300

$-0.10945300$

-0.10310400
0.53375300
0.07103900
-0.44393100
-0.60203800
-1.00467700
-1.12246600
-0.76406900
-0.77442700
-0.29184700
0.32545200
-0.28861400
-1.46262700
-2.04762900
-1.77383000
-0.32161500
0.92189600
1.40281400
2.09612500
3.01270600
2.32705900
1.45390600
0.22905000
-1.23165700
0.05267700
-0.65732900

\section{列}

.

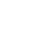

.

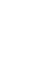


Methylene derived from truncated CuATSM<smiles></smiles>

\section{MN15/CBSB7+ Enthalpy= \\ MN15/CBSB7+ Free Energy=}

$-2904.400246$

$-2904.455058$

$\mathrm{O}^{3}$
$\mathrm{~S}$
$\mathrm{C}$
$\mathrm{N}$
$\mathrm{N}$
$\mathrm{C}$
$\mathrm{N}$
$\mathrm{N}$
$\mathrm{C}$
$\mathrm{S}$
$\mathrm{C}$
$\mathrm{N}$
$\mathrm{H}$
$\mathrm{Cu}$
$\mathrm{H}$
$\mathrm{H}$
$\mathrm{C}$
$\mathrm{H}$
$\mathrm{H}$
$\mathrm{H}$

$$
\begin{array}{r}
-1.44157300 \\
-2.57523900 \\
-2.27327900 \\
-0.97975800 \\
0.98563600 \\
1.56600700 \\
2.92945400 \\
3.29099800 \\
2.29298600 \\
-0.48347400 \\
-3.89136900 \\
-4.20887100 \\
0.37061900 \\
4.36442400 \\
1.56155000 \\
-1.21326500 \\
-2.29380700 \\
-0.72167900 \\
-4.55752100
\end{array}
$$$$
-1.86201400
$$$$
-0.57691200
$$$$
0.73728900
$$$$
0.94260800
$$$$
2.23357800
$$$$
1.08882600
$$$$
0.98122300
$$$$
-0.26786900
$$$$
-1.66383900
$$$$
2.23755600
$$$$
-0.85941700
$$$$
-1.81645600
$$$$
-0.49272700
$$$$
-0.43991100
$$$$
3.15661100
$$$$
3.37346500
$$$$
3.33199000
$$$$
4.33752700
$$

$-0.09963400$

$$
\begin{array}{r}
0.00000300 \\
0.00000400 \\
0.00000200 \\
0.00000100 \\
0.00000000 \\
0.00000000 \\
-0.00000100 \\
-0.00000400 \\
-0.00000300 \\
0.00000000 \\
0.00000200 \\
0.00000100 \\
-0.00000100 \\
-0.00000100 \\
-0.00000100 \\
0.00000000 \\
0.00000100 \\
0.00000000 \\
0.00000100
\end{array}
$$

Peroxyl-adduct derived from truncated CuATSM<smiles>COCC1=CN2N=CS[C@]23SC(N)=NN13</smiles>

CBS-QB3 Enthalpy=

CBS-QB3 Free Energy=

B3LYP/CBSB7 Enthalpy= B3LYP/CBSB7 Free Energy=

$$
\begin{array}{r}
2.28890700 \\
0.70836000 \\
-0.46041000 \\
-0.30784300 \\
-0.93912100 \\
0.31856800 \\
0.79426100 \\
2.09076000 \\
3.17314400 \\
-1.31288300 \\
0.63730200 \\
1.47038700 \\
1.48185600 \\
2.54379700 \\
-1.67887100
\end{array}
$$

\section{$-3092.076066$ $-3092.144469$ $-3095.064643$ $-3095.132779$}

$$
\begin{array}{r}
1.98613200 \\
2.72151900 \\
2.08814000 \\
0.76313100 \\
-1.47057100 \\
-1.72902300 \\
-3.00597800 \\
-3.01877600 \\
-1.67238300 \\
-0.05672200 \\
4.06306700 \\
4.61569500 \\
-0.13959700 \\
-4.00602900 \\
-2.25778800
\end{array}
$$

0.16618300

0.00116200

$-0.15571100$

$-0.15770700$

$-0.28829300$

$-0.14936600$

$-0.12857700$

0.01937800

0.17652100

$-0.29034500$

0.02852200

0.13271500

0.02456700

0.04656700

$-0.39010800$ 


$\begin{array}{lrrr}\mathrm{C} & -2.73159700 & 0.40560600 & -0.45833400 \\ \mathrm{H} & -2.87627300 & 1.35889700 & 0.05712000 \\ \mathrm{H} & -2.96219400 & 0.54905200 & -1.52247200 \\ \mathrm{H} & -0.26114500 & 4.50918100 & -0.07094300 \\ \mathrm{O} & -3.56572100 & -0.61478300 & 0.07573000 \\ \mathrm{O} & -4.92164300 & -0.26682800 & -0.33778800 \\ \mathrm{C} & -5.75242400 & -0.57924500 & 0.76719600 \\ \mathrm{H} & -5.50040800 & 0.02786500 & 1.64217100 \\ \mathrm{H} & -6.76071900 & -0.33903500 & 0.42298000 \\ \mathrm{H} & -5.69403000 & -1.64285500 & 1.01803500\end{array}$

Peroxyl-adduct derived from truncated CuATSM<smiles>COCC1=CN2N=CS[C@]23SC(N)=NN13</smiles>

\section{MN15/CBSB7+ Enthalpy= MN15/CBSB7+ Free Energy=}

$$
\begin{array}{r}
2.41134900 \\
0.89732200 \\
-0.31956500 \\
-0.24829800 \\
-1.04926600 \\
0.18973200 \\
0.57411300 \\
1.87164600 \\
3.03264100 \\
-1.31218700 \\
0.93623800 \\
1.82058200 \\
1.48442000 \\
2.26367500 \\
-1.84878800 \\
-2.68179200 \\
-2.81569600 \\
-2.81569600 \\
0.06898900 \\
-3.60614800 \\
-4.89155400 \\
-5.81921600 \\
-5.70508200 \\
-6.79791100 \\
-5.70507300
\end{array}
$$

$-3094.485531$ $-3094.552954$

$$
\begin{array}{rr}
1.85483100 & 0.00003100 \\
2.69396700 & -0.00000500 \\
2.14820300 & -0.00001600 \\
0.81399400 & -0.00001200 \\
-1.36734100 & 0.00001900 \\
-1.71203300 & 0.00004500 \\
-3.02100700 & 0.00004600 \\
-3.11581100 & 0.00000600 \\
-1.85000100 & -0.00005800 \\
0.07751500 & 0.00000600 \\
4.03897700 & -0.00001900 \\
4.52285000 & -0.00000500 \\
-0.20541100 & 0.00000500 \\
-4.12982700 & 0.00000900 \\
-2.10205200 & 0.00000600 \\
0.67861800 & 0.00001400 \\
1.31141400 & 0.88570200 \\
1.31143200 & -0.88566200 \\
4.55737500 & -0.00003800 \\
-0.38587300 & 0.00000200 \\
0.24241800 & -0.00000200 \\
-0.81906200 & -0.00002900 \\
-1.43422100 & 0.89639100 \\
-0.33975500 & -0.00002400 \\
-1.43418400 & -0.89647400
\end{array}
$$

\section{CuATSM (SMD, $\mathrm{H}_{2} \mathrm{O}$ )}<smiles>CNC1=NN2N=C(NC)S[C@]23SC(C)=C(C)N3C1</smiles>
MN15/CBSB7+ Enthalpy= 


$\begin{array}{lrrr}02 & & \\ \mathrm{Cu} & -0.00847400 & -0.69273600 & 0.00115200 \\ \mathrm{~S} & -1.86624200 & -1.97447000 & 0.11411900 \\ \mathrm{C} & -2.97474500 & -0.62008000 & 0.03075800 \\ \mathrm{~N} & -2.63541300 & 0.65629000 & -0.01004600 \\ \mathrm{~N} & -1.28878300 & 0.82910300 & -0.00104800 \\ \mathrm{C} & -0.73761600 & 2.00041500 & -0.00398200 \\ \mathrm{C} & 0.74806600 & 1.98704800 & 0.00475300 \\ \mathrm{~N} & 1.28038900 & 0.80730600 & -0.00009900 \\ \mathrm{~N} & 2.62493100 & 0.64017000 & 0.00788200 \\ \mathrm{C} & 2.96409400 & -0.63929700 & -0.03144100 \\ \mathrm{~S} & 1.84941100 & -1.98701000 & -0.11176600 \\ \mathrm{~N} & 4.27234900 & -0.94195000 & -0.01270300 \\ \mathrm{C} & 5.31559100 & 0.06480500 & 0.07175500 \\ \mathrm{H} & 5.21579400 & 0.65880700 & 0.98273300 \\ \mathrm{H} & 6.27842600 & -0.44117300 & 0.08390000 \\ \mathrm{H} & 5.28140500 & 0.74054300 & -0.78528000 \\ \mathrm{H} & 4.53004300 & -1.91963100 & -0.03469700 \\ \mathrm{C} & 1.56512200 & 3.23178200 & 0.01889900 \\ \mathrm{H} & 0.94540400 & 4.12594200 & 0.02452200 \\ \mathrm{H} & 2.21177000 & 3.24102100 & 0.90077000 \\ \mathrm{H} & 2.21961600 & 3.25716100 & -0.85683800 \\ \mathrm{C} & -1.48659700 & 3.28633900 & -0.01614400 \\ \mathrm{H} & -1.20244300 & 3.87747300 & -0.89126500 \\ \mathrm{H} & -2.55883900 & 3.10756600 & -0.03445600 \\ \mathrm{H} & -1.23350900 & 3.87632200 & 0.86932400 \\ \mathrm{~N} & -4.28457200 & -0.92328200 & 0.01161800 \\ \mathrm{C} & -5.32740300 & 0.08293300 & -0.07870700 \\ \mathrm{H} & -5.22652800 & 0.67380100 & -0.99170500 \\ \mathrm{H} & -6.29035300 & -0.42299500 & -0.09079500 \\ \mathrm{H} & -5.29557500 & 0.76204300 & 0.77580500 \\ \mathrm{H} & -4.54156000 & -1.90098800 & 0.03235900 \\ & & & \\ & & & \end{array}$

\section{CuATSM (SMD, $n$-octanol)}<smiles>CNC1=NN2C(C)=C(C)N3N=C(NC)SC23S1</smiles>

\section{MN15/CBSB7+ Enthalpy= MN15/CBSB7+ Free Energy= \\ $-3078.079531$ $-3078.149964$}

$$
\begin{array}{rr}
0.00865500 & -0.69731100 \\
1.86594700 & -1.97728600 \\
2.97168200 & -0.61990800 \\
2.63129800 & 0.65559500 \\
1.28667700 & 0.82726200 \\
0.73736300 & 1.99913300 \\
-0.74766000 & 1.98495200 \\
-1.27784500 & 0.80459200 \\
-2.62076300 & 0.63944300 \\
-2.96125700 & -0.63903700 \\
-1.84945800 & -1.98977700 \\
-4.27116300 & -0.93409200 \\
-5.30679900 & 0.07939400 \\
-5.20293900 & 0.67454600 \\
-6.27465000 & -0.41887000 \\
-5.26298300 & 0.75482300
\end{array}
$$

-0.00059300
-0.11321100
-0.02926600
0.01363000
0.00350100
0.00660300
-0.00700600
-0.00223300
-0.01168900
0.02984700
0.11155000
0.01170200
-0.06858400
-0.97927100
-0.07875000
0.78930300 
$-0.78370100$

$-0.03911000$

\section{CuGTSM (SMD, $\mathrm{H}_{2} \mathrm{O}$ )}<smiles>CNC1=NN2C=CN3N=C(NC)SC23S1</smiles>

\section{MN15/CBSB7+ Enthalpy= MN15/CBSB7+ Free Energy=}

$$
\begin{array}{r}
0.00000000 \\
-1.87593000 \\
-2.98419600 \\
-2.63790000 \\
-1.29817700 \\
1.29817700 \\
2.63790000 \\
2.98419600 \\
1.87593000 \\
4.29180400 \\
5.33473200 \\
5.26669500 \\
6.29828600 \\
5.26663300 \\
4.55387500 \\
-4.29180400 \\
-5.33473200 \\
-5.26669700 \\
-6.29828600 \\
-5.26663100 \\
-4.55387500 \\
-0.72947600 \\
-1.30221400 \\
0.72947600 \\
1.30221400
\end{array}
$$

$-2999.577241$ $-2999.638829$

$$
\begin{array}{r}
0.33989500 \\
1.61079900 \\
0.25570700 \\
-1.02510300 \\
-1.17803000 \\
-1.17803000 \\
-1.02510300 \\
0.25570700 \\
1.61079900 \\
0.55046600 \\
-0.46137400 \\
-1.09565400 \\
0.04309400 \\
-1.09560500 \\
1.52749900 \\
0.55046600 \\
-0.46137400 \\
-1.09563700 \\
0.04309400 \\
-1.09562200 \\
1.52749900 \\
-2.33653000 \\
-3.25874700 \\
-2.33653000 \\
-3.25874700
\end{array}
$$

\section{CuGTSM (SMD, $n$-octanol)}<smiles>CNC1=NN2C=CN3N=C(NC)SC23S1</smiles>

-0.00000100
0.00007000
0.00005900
0.00006100
0.00004800
-0.00003500
-0.00004800
-0.00006000
-0.00009300
-0.00004400
0.00001800
-0.88585400
0.00003800
0.88592000
-0.00004600
0.00003900
-0.00000500
0.88587900
-0.00003700
-0.88589500
0.00003900
0.00002700
0.00003200
-0.00000800
-0.00000800
MN15/CBSB7+ Enthalpy= MN15/CBSB7+ Free Energy= 
02

$\begin{array}{lrrr}\mathrm{Cu} & -0.00000100 & 0.33883900 & -0.00006500 \\ \mathrm{~S} & -1.86892500 & 1.61231100 & 0.10982300 \\ \mathrm{C} & -2.97706600 & 0.25823600 & 0.02598700 \\ \mathrm{~N} & -2.63290700 & -1.02197800 & -0.01649100 \\ \mathrm{~N} & -1.29545200 & -1.17849200 & -0.01219800 \\ \mathrm{~N} & 1.29545100 & -1.17849300 & 0.01218000 \\ \mathrm{~N} & 2.63290700 & -1.02198000 & 0.01650700 \\ \mathrm{C} & 2.97706700 & 0.25823400 & -0.02594100 \\ \mathrm{~S} & 1.86892800 & 1.61230700 & -0.10983800 \\ \mathrm{~N} & 4.28541600 & 0.54954200 & -0.00914600 \\ \mathrm{C} & 5.32269800 & -0.46362600 & 0.07161600 \\ \mathrm{H} & 5.27959100 & -1.13855300 & -0.78628100 \\ \mathrm{H} & 6.28929100 & 0.03675600 & 0.08150300 \\ \mathrm{H} & 5.21931600 & -1.05714300 & 0.98295400 \\ \mathrm{H} & 4.55122300 & 1.52510200 & -0.04241000 \\ \mathrm{~N} & -4.28541800 & 0.54954000 & 0.00929500 \\ \mathrm{C} & -5.32269800 & -0.46362800 & -0.07148600 \\ \mathrm{H} & -5.27941900 & -1.13871200 & 0.78627500 \\ \mathrm{H} & -6.28930100 & 0.03674000 & -0.08109600 \\ \mathrm{H} & -5.21947700 & -1.05697500 & -0.98295500 \\ \mathrm{H} & -4.55122000 & 1.52510500 & 0.04243900 \\ \mathrm{C} & -0.72982900 & -2.33825500 & -0.00490200 \\ \mathrm{H} & -1.30763900 & -3.25786600 & -0.00468700 \\ \mathrm{C} & 0.72982700 & -2.33825600 & 0.00484900 \\ \mathrm{H} & 1.30763700 & -3.25786700 & 0.00463000\end{array}$

\section{CuATSP (SMD, $\mathrm{H}_{2} \mathrm{O}$ )}<smiles>CC1=C(C)N2N=C(Nc3ccccc3)SC23SC(Nc2ccccc2)=NN13</smiles>

\section{MN15/CBSB7+ Enthalpy= MN15/CBSB7+ Free Energy=}

\section{$-3461.057904$ $-3461.144041$}
$-0.87814200$
$-2.14553600$
$-0.78168300$
0.48328200
0.64727700
1.82133100
1.80773500
0.62520200
0.46712100
$-0.80019900$
$-2.15829300$
$-1.13958000$
$-2.14001800$
3.04636600
3.94432100
3.08160500
3.03247900
3.10296300
3.68572100
2.92130200
3.70091100
$-1.12546600$
$-2.12654700$
0.00242700
0.12185400
0.07883000
0.04383900
0.02287600
0.01647200
$-0.01393100$
$-0.02142400$
$-0.04170100$
$-0.07634800$
$-0.11779300$
$-0.08361700$
$-0.09897200$
$-0.03158400$
$-0.06755900$
0.86022500
$-0.89669000$
0.03722500
$-0.85902800$
0.08329800
0.90011400
0.08577200
0.10018900 
5.45290900

6.66083600

5.48393300

7.87457000

6.63237700

6.71278200

4.56763600

7.91001400

8.79447100

6.72265900

8.85604700

$-5.46239500$

$-5.50013900$

$-6.66717800$

$-6.73144100$

$-4.58648600$

$-7.88363000$

$-6.63407500$

$-7.92545200$

$-6.74596700$

$-8.80079700$

$-8.87364100$
$-0.34946000$

$-1.05948200$

1.04928800

$-0.38999000$

$-2.14442300$

1.70849300

1.61672400

1.00496400

$-0.96025300$

2.79255300

1.53161800

$-0.34141700$

1.05697600

$-1.05647000$

1. 71114900

1.62807400

$-0.39217800$

$-2.14128000$

1.00260700

2.79516200

$-0.96649400$

1.52510600
-0.03119800
0.04497500
-0.05723000
0.09465300
0.06652900
-0.00540700
-0.11723400
0.07024300
0.15374200
-0.02604500
0.11000800
0.02914900
0.05592000
-0.05177700
-0.00024700
0.11964200
-0.10555800
-0.07362100
-0.08070300
0.02097000
-0.16826100
-0.12377400

\section{CuATSP (SMD, $n$-octanol)}

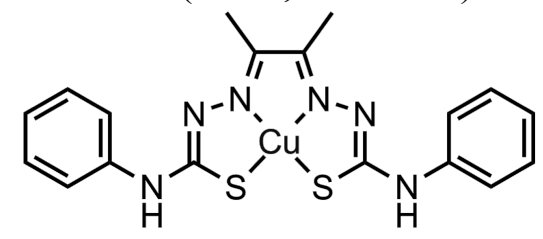

\section{MN15/CBSB7+ Enthalpy= MN15/CBSB7+ Free Energy=}

\section{$-3461.066731$ $-3461.151583$}
$-0.87327500$
$-2.14096700$
$-0.77702100$
0.48858900
0.65297400
1.82677700
1.81257000
0.63016900
0.47229600
$-0.79565300$
$-2.15375400$
$-1.13747300$
$-2.13874500$
3.04916800
3.95174600
3.06616400
3.04792000
3.10772100
3.69731700
2.92061900
3.70298300
$-1.12338400$
$-2.12530900$
$-0.35312400$
$-1.06641000$
1.04482000
$-0.40068700$
$-2.15126600$
0.00239500
0.12537700
0.08772100
0.05675900
0.03125000
0.02038500
$-0.01890700$
$-0.02988200$
$-0.05647700$
$-0.08645500$
$-0.12118800$
$-0.09272000$
$-0.10416500$
$-0.04384800$
$-0.04368000$
0.82499700
$-0.93270400$
0.04418700
$-0.84836700$
0.08447700
0.91303300
0.09308000
0.10264700
$-0.03548900$
0.06511700
$-0.07997500$
0.12147700
0.10095500 
6.71803800

4.57299200

7.91144300

8.78833300

6.73274600

8.85912800

$-5.46091200$

$-5.50441200$

$-6.66167300$

$-6.73695200$

$-4.59215600$

$-7.87982300$

$-6.62498700$

$-7.92698800$

$-6.75645600$

$-8.79445200$

$-8.87686700$
1.70002100
1.61222200
0.99330700
$-0.97322200$
2.78403800
1.51726800
$-0.34520400$
1.05220900
$-1.06336700$
1.70246900
1.62297800
$-0.40281400$
$-2.14799100$
0.99086600
2.78631900
$-0.97929800$
1.51067900

-0.02107400
-0.15973300
0.07952100
0.19998000
-0.05608100
0.12480000
0.03356200
0.08196500
-0.07272100
0.02077700
0.16637000
-0.13119900
-0.11106900
-0.08571600
0.05888400
-0.21415700
-0.13272600

$\mathrm{CuATSM}_{2}\left(\mathrm{SMD}, \mathrm{H}_{2} \mathrm{O}\right)$<smiles>CC1=C(C)N2N=C(N(C)C)SC23SC(N(C)C)=NN13</smiles>

\section{MN15/CBSB7+ Enthalpy=} MN15/CBSB7+ Free Energy=

\section{$-3156.532087$ $-3156.610290$}

$O{ }^{O}$
$\mathrm{Cu}$
$\mathrm{S}$
$\mathrm{C}$
$\mathrm{N}$
$\mathrm{N}$
$\mathrm{C}$
$\mathrm{C}$
$\mathrm{N}$
$\mathrm{N}$
$\mathrm{C}$
$\mathrm{S}$
$\mathrm{N}$
$\mathrm{C}$
$\mathrm{H}$
$\mathrm{H}$
$\mathrm{H}$
$\mathrm{C}$
$\mathrm{H}$
$\mathrm{H}$
$\mathrm{H}$
$\mathrm{C}$
$\mathrm{H}$
$\mathrm{H}$
$\mathrm{H}$
$\mathrm{N}$
$\mathrm{C}$
$\mathrm{H}$
$\mathrm{H}$
$\mathrm{H}$
$\mathrm{C}$
$\mathrm{H}$
$\mathrm{H}$
$\mathrm{H}$

\begin{abstract}
0.00807300
1.86687600

2.98813400

2.63346300

1.28986800

0.73676100

$-0.74698200$

$-1.28269900$

$-2.62350200$

$-2.97662500$

$-1.85004700$

$-4.29679700$

$-5.24736700$

$-5.04532200$

$-6.25113200$

$-5.19536200$

$-1.56467000$

$-0.94564800$

$-2.22538600$

$-2.20622500$

1.48774100

1.21211100

2.55985000

1.22993600

4.31386400

5.25168000

5.16257200

6.26333600

5.07098800

4.80767600

4.65001800

4.31830300

5.87551700
\end{abstract}

$-0.48867000$

$-1.76697400$

$-0.42233100$

0.85387100

1.02511300

2.19703400

2.18491600

1.00501500

0.84072200

$-0.44245100$

$-1.78130400$

$-0.71724900$

0.38847100

1.12543900

$-0.00744000$

0.89127600

3.42945900

4.32424800

3.44698200

3.44689800

3.48217000

4.06067700

3.30091400

4.08676500

$-0.69559700$

0.39569300

0.77537800

0.02419000

1.22084700

$-2.05368000$

$-2.39940100$

$-2.74901800$

$-2.06389200$
$-0.04288500$

$-0.15414100$

$-0.06838200$

0.00036700

$-0.01673100$

$-0.00113500$

$-0.00916300$

$-0.02275400$

$-0.02440100$

$-0.01716900$

0.05196000

$-0.06063600$

0.01965400

$-0.75751800$

$-0.12156100$

0.98983000

0.00026500

$-0.00590500$

$-0.87112400$

0.88608200

0.02554600

0.91186800

0.03321400

$-0.84863500$

$-0.07804400$

0.17093700

1.19378700

0.01764200

$-0.51671600$

0.11945700

1.14622200

$-0.56117900$

$-0.09261300$ 


\section{$\mathrm{CuATSM}_{2}$ (SMD, $n$-octanol)}

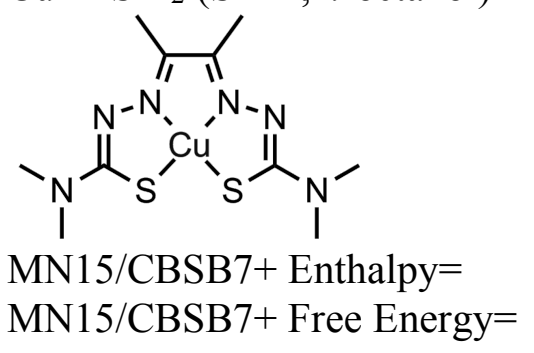

02

$\mathrm{Cu}$

$\mathrm{S}$

C

$\mathrm{N}$

$\mathrm{N}$

C

C

$\mathrm{N}$

$\mathrm{N}$

C

S

$\mathrm{N}$

C

$\mathrm{H}$

$\mathrm{H}$

$\mathrm{H}$

C

$\mathrm{H}$

$\mathrm{H}$

$\mathrm{H}$

C

$\mathrm{H}$

$\mathrm{H}$

$\mathrm{H}$

$\mathrm{N}$

C

$\mathrm{H}$

$\mathrm{H}$

$\mathrm{H}$

C

$\mathrm{H}$

$\mathrm{H}$

$\mathrm{H}$

C

$\mathrm{H}$

$\mathrm{H}$

$\mathrm{H}$

\begin{abstract}
0.00799500
1.86475100

2.98390700

2.62979400

1. 28866500

0.73647800

$-0.74621200$

$-1.28043500$

$-2.61952600$

$-2.97363800$

$-1.85084400$

$-4.29324500$

$-5.23517000$

$-5.03760200$

$-6.24480600$

$-5.16234200$

$-1.57275800$

$-0.96221300$

$-2.22583900$

$-2.22648400$

1. 49488700

1.21953300

2.56569900

1.25354100

4.30689600

5.24308400

5.12771800

6.25766200

5.08115100

4.81127300

4.63353500

4.34493200

5.88414500

$-4.80109100$

$-4.32638300$

$-4.63436800$

$-5.87184700$
\end{abstract}

\section{$-3156.538343$ \\ $-3156.617111$}

$$
\begin{array}{rr}
-0.49231100 & -0.01940400 \\
-1.77140100 & -0.12817000 \\
-0.42381000 & -0.04983100 \\
0.85405300 & 0.00653800 \\
1.02447200 & -0.00667200 \\
2.19682500 & 0.00302900 \\
2.18347200 & -0.00797400 \\
1.00299700 & -0.01158100 \\
0.84078000 & -0.01932900 \\
-0.44199500 & 0.00123000 \\
-1.78397000 & 0.08226100 \\
-0.71041100 & -0.03367700 \\
0.40383200 & -0.02702600 \\
1.08014200 & -0.86086500 \\
0.00807400 & -0.12180100 \\
0.97923600 & 0.90045600 \\
3.42290500 & -0.01542000 \\
4.32419200 & -0.02089000 \\
3.42837700 & -0.89343400 \\
3.43984700 & 0.86205400 \\
3.47900600 & 0.02515600 \\
4.06815200 & 0.90496900 \\
3.28690500 & 0.04383500 \\
4.07913000 & -0.85729500 \\
-0.69220900 & -0.04367300 \\
0.41311700 & 0.12870100 \\
0.88353400 & 1.11016700 \\
0.02910600 & 0.03546000 \\
1.17670300 & -0.63376700 \\
-2.05586400 & 0.04346200 \\
-2.49459600 & 1.03073800 \\
-2.69344400 & -0.70844500 \\
-2.03869400 & -0.14240400 \\
-2.07111400 & 0.08076200 \\
-2.72832100 & -0.64954800 \\
-2.48432100 & 1.08027500 \\
-2.05707700 & -0.11601700
\end{array}
$$

\section{Coordination of peroxyl to the $\mathrm{Cu}$-ion of CuATSM}




$$
\text { MN15/CBSB7+ Enthalpy= }
$$

\section{$-3268.094309$ \\ $-3268.181300$}

$\begin{array}{lrrr}\text { O }^{3} & & & \\ \mathrm{C} & 2.78307500 & -0.14067700 & -0.75134400 \\ \mathrm{~N} & 2.38617600 & 1.05667400 & -0.36420100 \\ \mathrm{~N} & 1.03849400 & 1.13818100 & -0.26031000 \\ \mathrm{C} & 0.43703600 & 2.21532000 & 0.12870900 \\ \mathrm{C} & -1.03933800 & 2.09654400 & 0.22487800 \\ \mathrm{~N} & -1.50551900 & 0.92045100 & -0.04669400 \\ \mathrm{~N} & -2.83088400 & 0.66078500 & 0.00941300 \\ \mathrm{C} & -3.09841800 & -0.59658500 & -0.30415800 \\ \mathrm{~S} & -1.92156600 & -1.81554900 & -0.74492800 \\ \mathrm{~N} & -4.38623900 & -0.98180500 & -0.28727400 \\ \mathrm{C} & -5.47414500 & -0.08476100 & 0.05832100 \\ \mathrm{H} & -5.50912700 & 0.76519100 & -0.62669400 \\ \mathrm{H} & -6.41022700 & -0.63549100 & -0.00637500 \\ \mathrm{H} & -5.35314500 & 0.30000500 & 1.07334000 \\ \mathrm{H} & -4.58725500 & -1.94264800 & -0.52536500 \\ \mathrm{C} & -1.92666100 & 3.23032400 & 0.61581800 \\ \mathrm{H} & -1.37324000 & 4.15871200 & 0.74188800 \\ \mathrm{H} & -2.69993500 & 3.37038100 & -0.14339900 \\ \mathrm{H} & -2.44250900 & 2.99223700 & 1.55018400 \\ \mathrm{C} & 1.13868000 & 3.48474200 & 0.47672300 \\ \mathrm{H} & 0.88572100 & 3.79314700 & 1.49455700 \\ \mathrm{H} & 2.21467900 & 3.35013500 & 0.39647600 \\ \mathrm{H} & 0.82617400 & 4.28926500 & -0.19483700 \\ \mathrm{~N} & 4.10847700 & -0.35376400 & -0.86219200 \\ \mathrm{C} & 5.09600400 & 0.67932800 & -0.60767100 \\ \mathrm{H} & 5.00364800 & 1.05805400 & 0.41231300 \\ \mathrm{H} & 6.08722400 & 0.25120100 & -0.74189000 \\ \mathrm{H} & 4.97175300 & 1.51909600 & -1.29550400 \\ \mathrm{H} & 4.40750500 & -1.26256400 & -1.18598900 \\ \mathrm{~S} & 1.74446300 & -1.49826500 & -1.13780100 \\ \mathrm{Cu} & -0.15034500 & -0.43143800 & -0.53795100 \\ \mathrm{O} & 0.56610200 & -0.63551000 & 2.01707600 \\ \mathrm{O} & 0.81523600 & -1.86911100 & 2.35538900 \\ \mathrm{C} & 2.22080900 & -2.07281900 & 2.58554300 \\ \mathrm{H} & 2.32297100 & -3.10850400 & 2.89992800 \\ \mathrm{H} & 2.74989900 & -1.89208500 & 1.64871800 \\ \mathrm{H} & 2.55305000 & -1.38858800 & 3.36504900\end{array}$

Peroxyl adduct from addition to $\mathrm{Cu}$-atom of CuATSM<smiles>CNC1=NN2C(C)=C(C)N3N=C(NC)SC23S1</smiles>

MN15/CBSB7+ Enthalpy= 


$\begin{array}{lrrr}\mathrm{O}^{1} & & \\ \mathrm{C} & 2.84345300 & -0.49406900 & -0.61472200 \\ \mathrm{~N} & 2.54894900 & 0.73691600 & -0.26518500 \\ \mathrm{~N} & 1.20763800 & 0.91937500 & -0.17115000 \\ \mathrm{C} & 0.65811600 & 2.09907200 & -0.13414000 \\ \mathrm{C} & -0.81374200 & 2.10329900 & -0.13338800 \\ \mathrm{~N} & -1.33226400 & 0.95707800 & -0.45019500 \\ \mathrm{~N} & -2.66274900 & 0.73613000 & -0.41039500 \\ \mathrm{C} & -2.92741000 & -0.55300100 & -0.44169400 \\ \mathrm{~S} & -1.71647600 & -1.83636300 & -0.49217900 \\ \mathrm{~N} & -4.20459800 & -0.96250400 & -0.44831000 \\ \mathrm{C} & -5.31942500 & -0.03029100 & -0.46418500 \\ \mathrm{H} & -5.27697300 & 0.60452500 & -1.35092300 \\ \mathrm{H} & -6.24590700 & -0.59985800 & -0.47534800 \\ \mathrm{H} & -5.29898900 & 0.61035900 & 0.42038200 \\ \mathrm{H} & -4.38757000 & -1.95374000 & -0.37886800 \\ \mathrm{C} & -1.63694800 & 3.29809400 & 0.21483600 \\ \mathrm{H} & -1.02284300 & 4.11752600 & 0.58454000 \\ \mathrm{H} & -2.19818300 & 3.64154900 & -0.65831400 \\ \mathrm{H} & -2.36695100 & 3.02102000 & 0.97869700 \\ \mathrm{C} & 1.45055400 & 3.36101700 & -0.10947200 \\ \mathrm{H} & 1.45971000 & 3.77232200 & 0.90481500 \\ \mathrm{H} & 2.47681100 & 3.16903200 & -0.41383000 \\ \mathrm{H} & 0.99871100 & 4.10668600 & -0.76592600 \\ \mathrm{~N} & 4.13040600 & -0.84053700 & -0.77375000 \\ \mathrm{C} & 5.21739400 & 0.10756400 & -0.59917900 \\ \mathrm{H} & 5.22469300 & 0.50321500 & 0.41873900 \\ \mathrm{H} & 6.15788000 & -0.40343600 & -0.79192500 \\ \mathrm{H} & 5.11343700 & 0.94422200 & -1.29329700 \\ \mathrm{H} & 4.33684000 & -1.79922600 & -1.01618100 \\ \mathrm{~S} & 1.64509000 & -1.74439400 & -0.91960000 \\ \mathrm{Cu} & -0.02423200 & -0.51533400 & -0.15402400 \\ \mathrm{O} & -0.55479800 & -0.19200700 & 1.78623400 \\ \mathrm{O} & 0.00012600 & -1.18503600 & 2.60837100 \\ \mathrm{C} & 1.29570500 & -0.77121900 & 2.97375800 \\ \mathrm{H} & 1.70471500 & -1.55046300 & 3.61920000 \\ \mathrm{H} & 1.92891800 & -0.65922100 & 2.08521900 \\ \mathrm{H} & 1.26012600 & 0.18062500 & 3.51335500\end{array}$

\section{Peroxyl adduct from addition to S-atom of CuATSM}

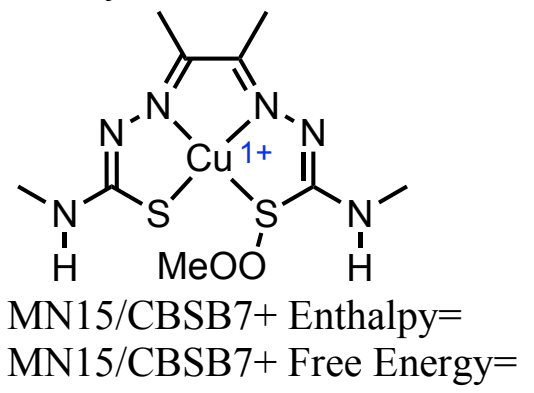

\section{$-3268.050386$ \\ $-3268.132313$}

03
$\mathrm{Cu}$
$\mathrm{C}$
$\mathrm{N}$
$\mathrm{N}$
$\mathrm{C}$
$\mathrm{C}$
$\mathrm{N}$
$\mathrm{N}$
$\mathrm{C}$

\begin{abstract}
$-0.23652500$
2.82902700

2.28049900

0.95615100

0.33104200

$-1.07663900$

$-1.55868200$

$-2.89185900$

$-3.17335700$
\end{abstract}
$-0.46928000$
$-0.11637400$
1.03622100
1.05945600
2.24656700
2.14896200
0.90970500
0.65950000
$-0.59786900$

-0.30352200
-0.56858400
-0.35178500
-0.13189400
0.06291300
0.15256000
-0.01238800
0.00413700
-0.22607800 
$-1.85083800$

$-0.98677500$

$-0.06601800$

0.75127000

$-0.60716400$

0.37148200

$-1.96653600$

3. 28143300

4. 22840200

3. 37719700

3.08278100

3. 50263300

4. 37274500

3. 42172300

3.67042600

$-0.28301300$

0.89280900

1. 42112200

0.57566700

1.59095100

$-1.05241700$

$-1.61059700$

$-2.27475200$

$-1.66525700$

$-0.87107500$

$-0.48130300$

$-1.49024800$

$-0.04602500$
-0.53059500
-0.24433900
0.01627500
-0.70965900
-0.05780700
1.01535900
-0.38720800
0.39356900
0.52861000
-0.44458000
1.28182500
0.14781900
0.34631900
0.93826500
-0.78561900
-0.63863800
-0.59198500
0.34998400
-0.64983500
-1.40917000
-1.21739700
-0.73004900
0.78779100
1.63199800
2.59739600
3.23708900
3.18199500
2.11700800

\section{Alkoxyl-Cu complex derived from CuATSM}

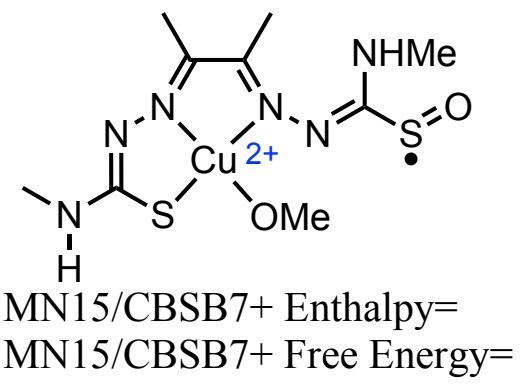

03

C

$\mathrm{N}$

$\mathrm{N}$

C

C

$\mathrm{N}$

$\mathrm{N}$

C

S

$\mathrm{N}$

C

$\mathrm{H}$

$\mathrm{H}$

$\mathrm{H}$

$\mathrm{H}$

C

$\mathrm{H}$

$\mathrm{H}$

$\mathrm{H}$

C

$\mathrm{H}$

\begin{abstract}
$-2.90190700$
$-2.26031900$

$-0.91491100$

$-0.21966300$

1.23760700

1.65049200

2. 96280300

3.20590200

2.00858000

4.48057100

5.57979000

5.42661000

6.49983900

5.67166300

4.66445000

2.16566500

1.62804100

2.83022700

2.80126600

$-0.80831500$

$-0.18948200$
\end{abstract}

$-3268.142260$

$-3268.226383$

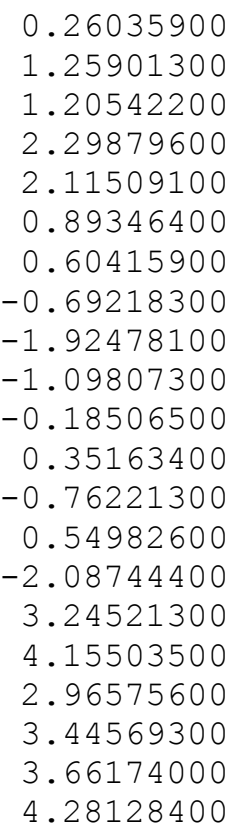

0.26035900

0.21036200

$-0.32685100$

$-0.07963700$

$-0.07481700$

0.09266800

$-0.03270700$

0.11442800

$-0.00417800$

$-0.30933700$

0.12309200

0.38146300

1. 32022100

0.44499900

$-0.42111400$

0.03507300

0.38661000

0.64724800

1.20654300

$-0.48118600$

$-0.21740700$

$-0.86893500$ 


$\begin{array}{lrrr}\mathrm{H} & -1.81751100 & 3.59189300 & -0.61782700 \\ \mathrm{H} & -0.85673200 & 4.15488300 & 0.75853300 \\ \mathrm{~N} & -4.18102000 & -0.01024500 & -0.04146100 \\ \mathrm{C} & -4.93635500 & 0.72222900 & -1.04162100 \\ \mathrm{H} & -4.43147600 & 0.67667500 & -2.00902700 \\ \mathrm{H} & -5.92462500 & 0.27735800 & -1.12904400 \\ \mathrm{H} & -5.03921100 & 1.77150100 & -0.75607600 \\ \mathrm{H} & -4.58561900 & -0.79989700 & 0.45131300 \\ \mathrm{~S} & -2.14661900 & -0.77617300 & 1.45711300 \\ \mathrm{O} & -3.16232400 & -1.84874100 & 1.70907200 \\ \mathrm{Cu} & 0.28531800 & -0.47146900 & -0.39878700 \\ \mathrm{O} & -1.15311100 & -1.51012800 & -0.97095400 \\ \mathrm{C} & -1.14413600 & -2.90416100 & -0.89374400 \\ \mathrm{H} & -1.10199500 & -3.26220100 & 0.14839200 \\ \mathrm{H} & -2.06114400 & -3.31004000 & -1.34007600 \\ \mathrm{H} & -0.29124000 & -3.34920400 & -1.42767300\end{array}$




\section{References}

1. Gingras, B. A.; Suprunchuk, T.; Bayley, C. H. Preparation of Some Thiosemicarbazones and Their Copper Complexes Part III. Can. J. Chem. 1962, 40 (6), 1053-1059.

2. Sirois, J. J.; Padgitt-Cobb, L.; Gallegos, M. A.; Beckman, J. S.; Beaudry, C. M.; Hurst, J. K. Oxidative Release of Copper From Pharmacologic Copper Bis(Thiosemicarbazonato) Compounds. Inorg. Chem. 2018, 57 (15), 8923-8932.

3. Beraldo, H.; Boyd, L. P.; West, D. X. Copper(II) and Nickel(II) Complexes of Glyoxaldehyde Bis $\{\mathrm{N}(3)-$ Substituted Thiosemicarbazones\}. Transit. Met. Chem. 1997, 23 (1), 67-71.

4. Petering, H. G.; Buskirk, H. H.; Underwood, G. E. The Anti-Tumor Activity of 2-Keto-3Ethoxybutyraldehyde Bis(Thiosemicarbazone) and Related Compounds. Cancer Res. 1964, 24, 367-372. 5. Dearling, J. L. J.; Lewis, J. S.; Mullen, G. E. D.; Welch, M. J.; Blower, P. J. Copper Bis(Thiosemicarbazone) Complexes as Hypoxia Imaging Agents: Structure-Activity Relationships. J. Biol. Inorg. Chem. 2002, 7 (3), 249-259.

6. Niederschulte, U.; Ballschmiter, K. Analytical Application of 1,2-Diketo-bis-(4-Phenyl)Thiosemicarbazones. Z. Anal. Chem. 1972, 261, 191-197.

7. Yin, H.; Hachey, D. L.; Porter, N. A. Analysis of Diacyl Peroxides by $\mathrm{Ag}^{+}$Coordination Ionspray Tandem Mass Spectrometry: Free Radical Pathways of Complex Decomposition. J. Am. Soc. Mass Spectrom. 2001, 12, 449-455.

8. Seal, J. R.; Havrilla, C. M.; Porter, N. A. Analysis of Unsaturated Compounds by $\mathrm{Ag}^{+}$Coordination Ionspray Mass Spectrometry: Studies of the Formation of the $\mathrm{Ag}^{+} /$Lipid Complex. J. Am. Soc. Mass Spectrom. 2003, 14, 872-880. 